DETERMINING THE CHARACTERISTICS OF TRANSITION-BASED INTERVENTIONS MOST EFFECTIVE IN ENHANCING QUALITY OF CARE FOR SENIORS: A SYSTEMATIC REVIEW

\author{
by \\ Sarah Rosato \\ BScN, Western University, 2014 \\ A thesis \\ presented to Ryerson University \\ in partial fulfillment of the \\ requirements for the degree of \\ Master in Nursing \\ in the Program of \\ Nursing
}

Toronto, Ontario, Canada, 2019

(C) Sarah Rosato, 2019 


\section{AUTHOR'S DECLARATION FOR ELECTRONIC SUBMISSION OF A THESIS}

I hereby declare that I am the sole author of this thesis. This is a true copy of the thesis, including any required final revisions, as accepted by my examiners.

I authorize Ryerson University to lend this thesis to other institutions or individuals for the purpose of scholarly research.

I further authorize Ryerson University to reproduce this thesis by photocopying or by other means, in total or in part, at the request of other institutions or individuals for the purpose of scholarly research.

I understand that my thesis may be made electronically available to the public. 


\title{
DETERMINING THE CHARACTERISTICS OF TRANSITION-BASED INTERVENTIONS \\ MOST EFFECTIVE IN ENHANCING QUALITY OF CARE FOR SENIORS: A \\ SYSTEMATIC REVIEW
}

\author{
Abstract \\ Sarah Rosato \\ Master of Nursing \\ Ryerson University, Toronto, 2019
}

Introduction: Seniors (65 years or older) often require additional support and resources during the transition from acute care to home. A comprehensive understanding of the transition-based literature will support the development and implementation of effective interventions, possibly resulting in organizational and individual benefits.

Purpose: A systematic review was conducted to identify the characteristics of transition-based interventions most effective in enhancing quality of care for seniors transitioning from hospital to home.

Methods: Primary research that evaluated a transitional care intervention for seniors and measured one of more quality of care outcome were included. Chi-square test for independence, ANOVA, and descriptive analysis were used.

Results: Forty-six interventions were reviewed for their specific characteristics. Multicomponent interventions which used multiple delivery methods (face-to-face/telephone), over one-to-three months $(p=<0.05)$, were most effective in enhancing quality of care.

Implications/Conclusions: Understanding the most effective intervention characteristics may support the provision of effective/efficient transitional care for seniors moving from acute care to home. 


\section{ACKNOWLEDGEMENTS}

I would like to begin by expressing my deepest appreciation to my supervisors, Dr. Suzanne Fredericks and Charlotte Lee, for your tireless support and guidance throughout this process. I feel privileged to have learned from your expertise in the fields of nursing, research, and teaching.

To my committee member, Beth McCay, thank you for the countless hours you spent helping me achieve my professional goals. Your prompt and useful advice was invaluable to the success of this project.

Lastly, I would like to thank my friends and family for their unconditional love and support, which gave me the courage to overcome any challenges faced during these years. 


\section{TABLE OF CONTENTS}

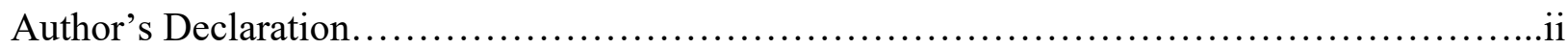

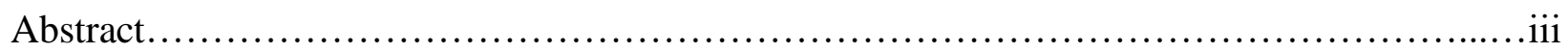

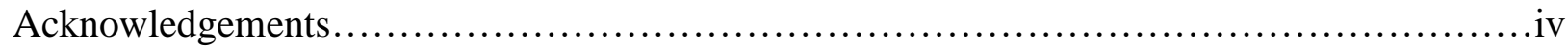

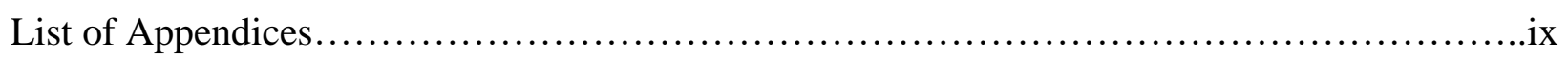

CHAPTER 1: INTRODUCTION .......................................................

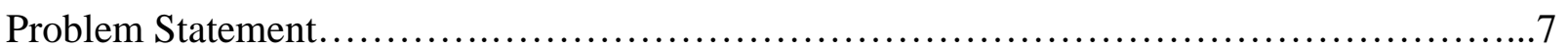

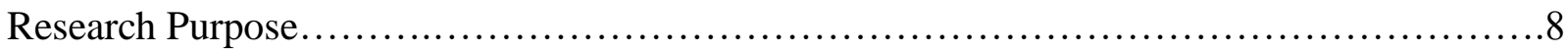

Significance of the Review....................................................

CHAPTER 2: EMPIRICAL LITERATURE REVIEW ............................... 10

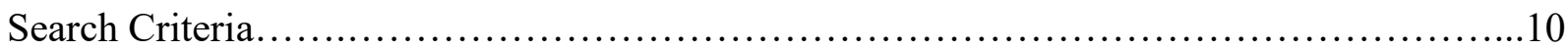

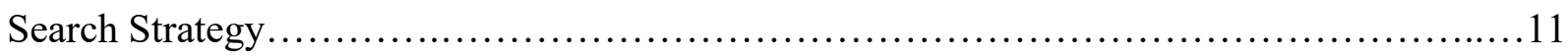

Characteristics of Included Reviews.............................................. 16

Intervention Characteristics: Component, Mode, Dose, Approach.........................17

Research Findings.............................................................. 19

Critique of Empirical Evidence................................................21

CHAPTER 3: CONCEPTUAL FRAMEWORK.......................................24

Transitional Care Interventions.................................................24

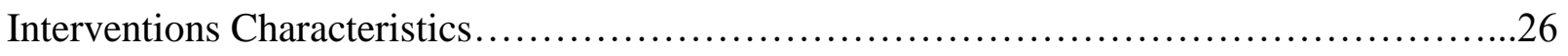

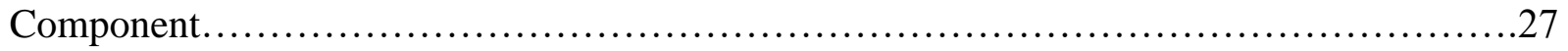

Mode...................................................................... 30

Dose......................................................................

Approach................................................................. 32 
Outcome of Interest: Quality of Care.

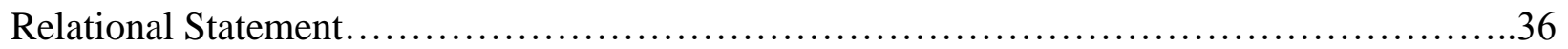

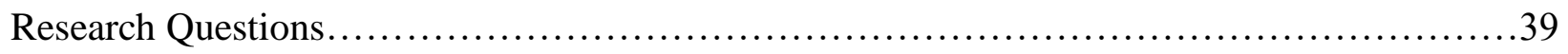

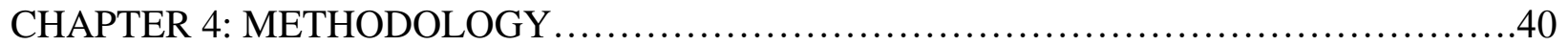

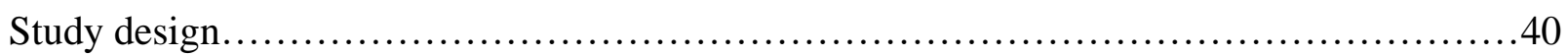

Selection Criteria.............................................................. 41

Inclusion Criteria .........................................................41

Exclusion Criteria.........................................................43

Critical Appraisal Tool.........................................................43

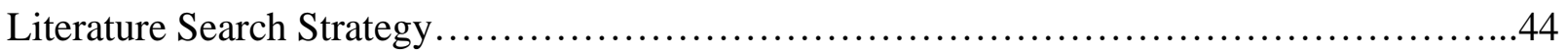

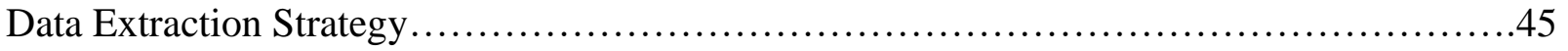

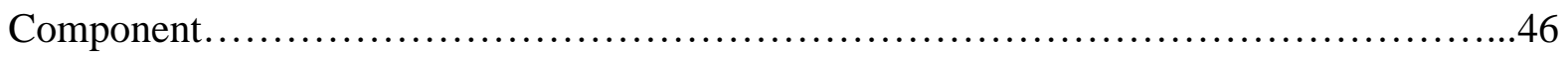

Mode........................................................................46

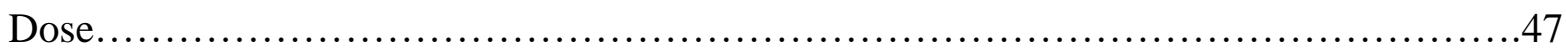

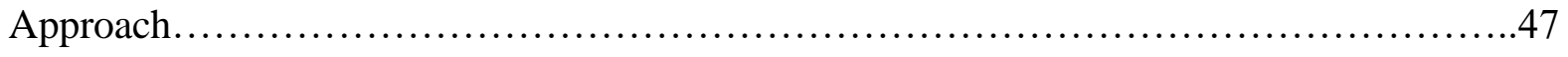

Outcomes of Transitional Care Interventions: Quality of Care $\ldots \ldots \ldots \ldots \ldots \ldots \ldots \ldots \ldots \ldots . . . .48$

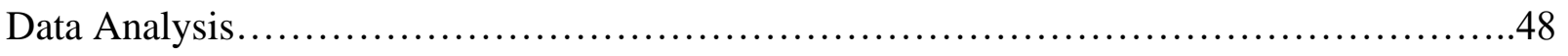

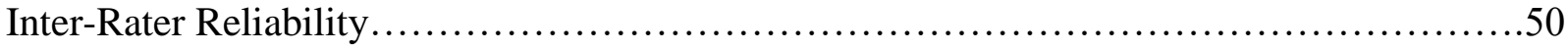

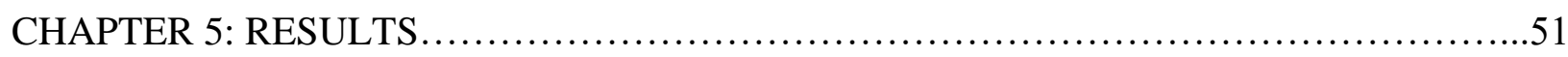

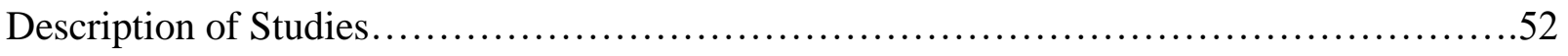

Description of Participants......................................................52

Patterns Across Intervention Characteristics.......................................52

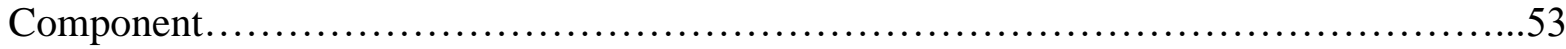


Mode

Dose.

Approach .55

Indicators of Quality of Care .55

Primary Research Question.................................................................56

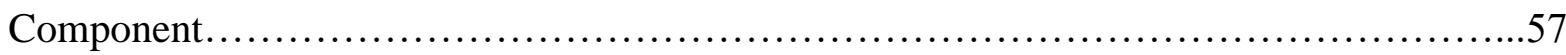

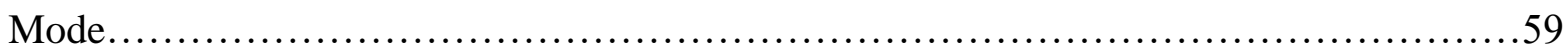

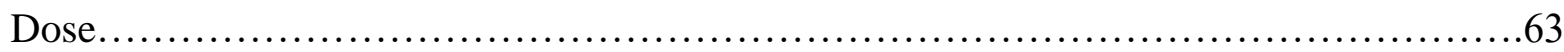

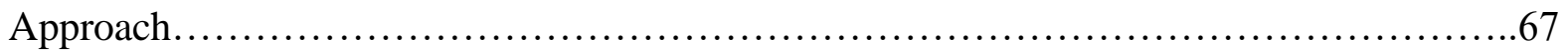

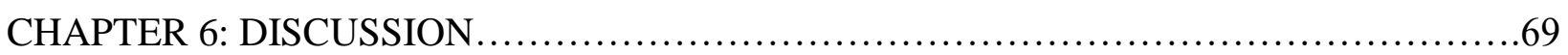

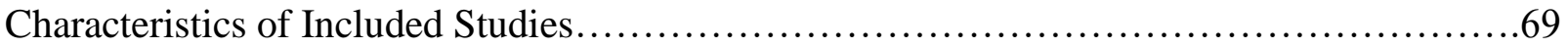

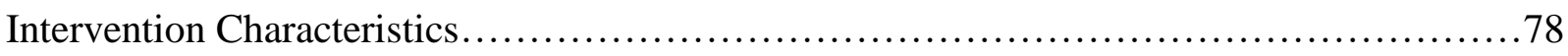

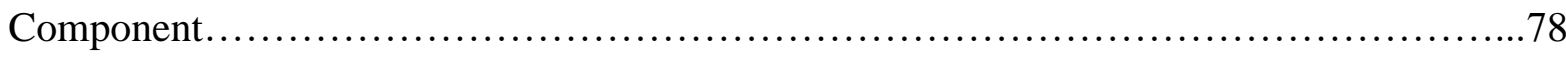

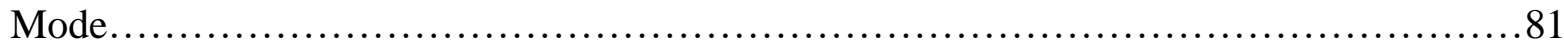

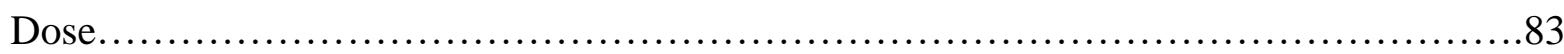

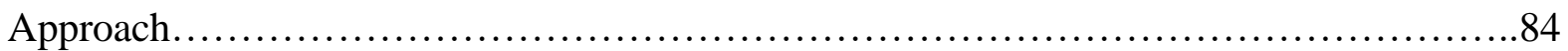

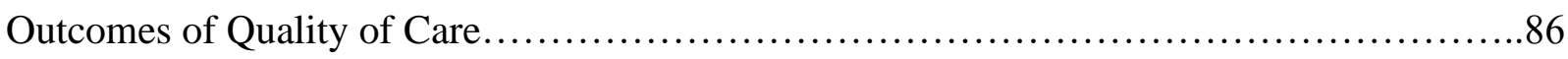

Summary of the Effects of Intervention Characteristics on Outcomes.........................86

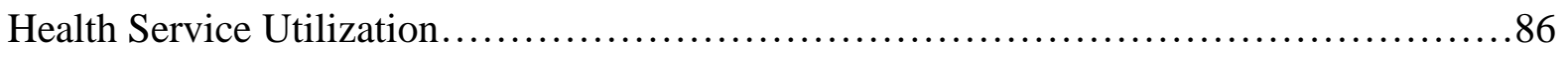

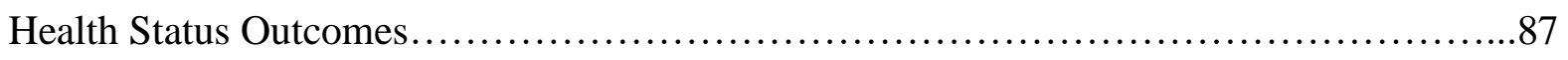

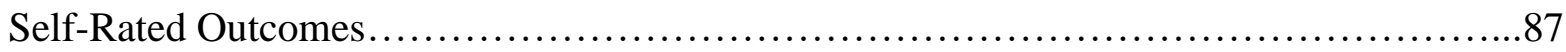

CHAPTER 7: IMPLICATIONS, LIMITATIONS, CONCLUSION $\ldots \ldots \ldots \ldots \ldots \ldots \ldots \ldots \ldots . \ldots 8$

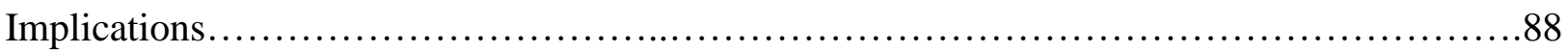




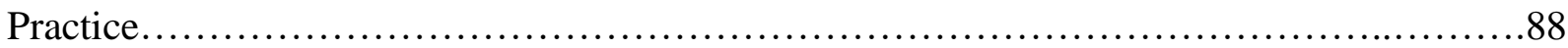

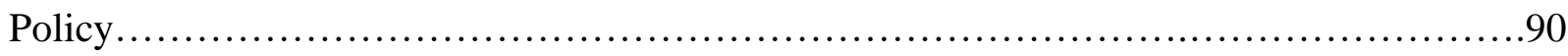

Research..................................................................... 92

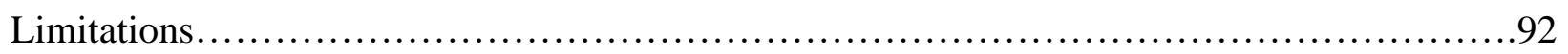

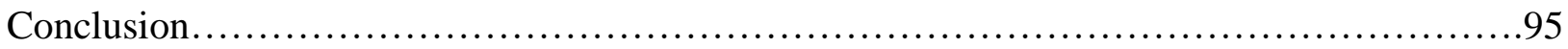

Appendices.................................................................... 98

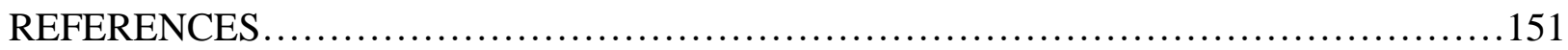




\section{LIST OF APPENDICES}

Appendix A: Literature Review Summary ........................................... 98

Appendix B: PRISMA Flow Diagram.............................................. 100

Appendix C: PRISMA Checklist for Systematic Reviews....................................101

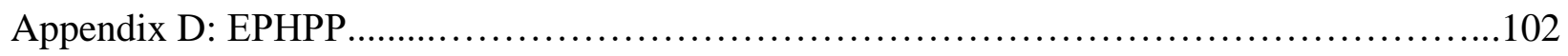

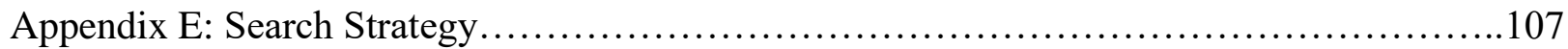

Appendix F: Table of Included Studies................................................. 108

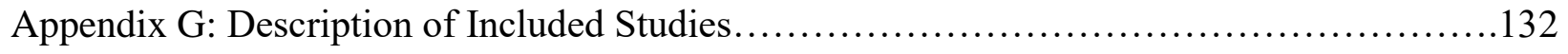

Appendix H: Description of Intervention Characteristics.................................132

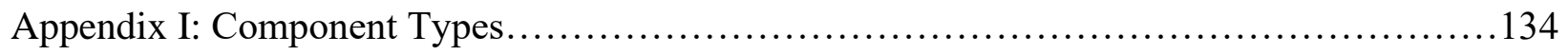

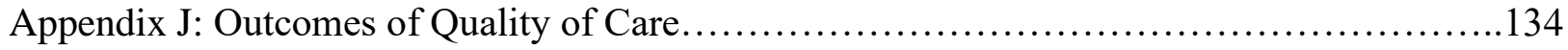

Appendix K: Combinations of Outcomes............................................. 135

Appendix L: An Overview of Studies Yielding Statistical Significance Based on Intervention

Characteristic

Appendix M: An Overview of Studies Yielding Non-Significance Findings Based on

Intervention Characteristic ........................................................

Appendix N: An Overview of Studies Yielding Variable Findings Based on Intervention

Characteristics.

Appendix O: A Breakdown of Intervention Characteristics That Used A Combination of

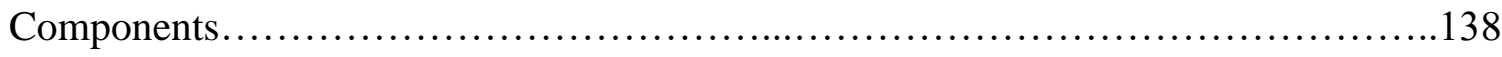

Appendix P: A breakdown of intervention characteristics with A combination of Modes.......139

Appendix Q: Chi-Square Results................................................. 140

Appendix R: Analysis of Variance Results................................................150 


\section{Chapter One}

\section{Introduction}

The number of seniors, considered in most developed countries to be individuals aged 65 years and over, is continually rising (Canadian Medical Association [CMA], 2016; Fuster, 2017; World Health Organization, 2018). In 2016, statistics Canada reported 15\% of the population was over 65 years of age; this demonstrates a 7.4\% increase since the year 1960 (CMA, 2016). The CMA (2016) projects, by 2024, the number of senior Canadians (65 and older) will increase to account for $20.1 \%$ of the national population. Within this population, the number of older seniors (those age 75 and older), who tend to rely more on the health care system, is expected to double in size (Canadian Institute for Health Information [CIHI], 2017).

One effect of the aging population is the increased prevalence of people living with chronic conditions that often require considerable acute care resources (Allen et al., 2014; CIHI, 2011). As many as $75-80 \%$ of Canadian senior's report having one or more chronic conditions (CIHI, 2011). The most frequently reported chronic conditions among seniors include high blood pressure (47\%), arthritis (27\%), heart disease (19\%), diabetes (17\%), and cancer (12\%) (CIHI, 2011). The CIHI (2011) reports that older seniors (age 75 and older) are more likely than younger seniors (age 65 to 74 ) to have at least three chronic conditions (36\% and 20\%, respectively), (CIHI, 2011). Regardless of age group (65 to 74 or 75 and older), seniors with three or more chronic conditions had three times more acute care visits than seniors with no chronic conditions (CIHI, 2011).

In addition to the onset and/or exacerbation of chronic illness, seniors often experience multifactorial health and psychosocial care needs (CMA, 2016). Seniors are at a greater risk of issues related to accessing appropriate and timely preventative primary care (CMA, 2016). As a 
result, many seniors are not getting the necessary primary health care to prevent or delay the onset of chronic conditions, nor are they receiving adequate secondary preventative care to avoid comorbidity and other complications (CIHI, 2011). Additionally, seniors often experience other factors in relation to complex care and comorbid conditions, such as financial deficits, polypharmacy, receiving services from multiple practitioners, and requiring assistance from a caregiver (CMA, 2016). The complexity of these issues may contribute to frequent, often preventable, visits to an acute care facility; over $37 \%$ of Canadian seniors report visiting an emergency department for a condition that could have been treated by their primary physician (CMA, 2016).

The multifaceted psychosocial care needs of the aging population, as well as systembased barriers to care, may lead to frequent use of acute care resources (CMA, 2016). The acute health care system, defined as "the most time-sensitive, individually-oriented diagnostic and curative actions whose primary purpose is to improve health" was designed to rapidly assess, diagnosis, and treat episodic health issues (Hirshon et al., 2013, p. 386). Today the Canadian healthcare system struggles to care for the growing number of medically complex older adults (CMA, 2016; Wong, Ryan, \& Liu, 2014). Due to the demographic changes that are projected to continue over the next two decades, many acute care institutions are focused on preparing to meet the health care needs of the aging population (CIHI, 2017).

As demonstrated, the extent to which the population is aging will have a significant impact on the healthcare system, requiring an increased emphasis on geriatric care interventions and protocols (CMA, 2016; Allen, Hutchinson, Brown, \& Livingston, 2014). To decrease high healthcare utilization and address the psychosocial needs of the aging population, many innovative strategies have emerged which aim to improve integration and continuity across the 
health care spectrum (Kim \& Thyer, 2015; Naylor, 2012). Such strategies are referred to as 'transitional care' (Naylor, 2012). Transitional care is defined as "a set of actions designed to ensure the coordination and continuity of health care as patients transfer between different locations or different levels of care within the same location" (Coleman, Boult, \& American Geriatrics Society Health Care Systems Committee, 2003, p. 549). Ideally, transition-based care involves a comprehensive care plan derived by healthcare providers who are well-versed in the patient's goals, preferences, and psychosocial health status (Coleman et al., 2003). Optimal transitional care is essential for those with complex care needs, such as the senior population and their caregivers (Coleman et al., 2003; Naylor, 2012).

Transitional care includes a broad range of services that ensure continuity and promote a safe and timely transfer of care (Burke, Prochazka, \& Misky, 2013; Naylor, 2012). Within their ideal transition in care model, authors Burke et al. (2013) conceptualize transitional care using ten domains. Each domain emphasizes the importance of accounting for patient preferences and ensuring continual communication between providers to help achieve optimal care (Burke et al., 2013). Transition-based strategies extend from the acute care setting, such as planning for discharge while the patient is still being treated in the hospital, to additional support in the community, such as monitoring symptoms after discharge (Burke et al., 2013). Overall, the ideal transition in care model provides insight into how to implement evidence-informed practice, while addressing certain systemic health care issues often associated with the senior population (e.g. readmission rates) (Burke et al., 2013).

Transitional care is not strictly defined by beginning (e.g. during hospital stay) and end points (e.g. once the senior returns home) and includes pre-hospital discharge care activities and post-discharge interventions (Allen, et al., 2014). Transition-destinations for seniors vary and 
include returning home, arranging for rehabilitation services, long-term care, retirement living, or being discharged to another hospital (Coleman et al., 2003; DeCoster, Ehlman, \& Conners, 2013). Supporting the transition from acute care to home is particularly important, as it can preserve health care resources and uphold patient-centred measures of quality (CIHI, 2017).

Patients over 65 occupy acute care inpatient beds about 1.5 times longer in overall length of stay when compared to younger Canadians (CMA, 2013). According to the CMA (2013), prolonged hospital stays for seniors are often due to non-acute reasons, such as the presence of comorbid conditions which complicate care, lack of home care resources, or the availability of an informal caregiver. Multiple and prolonged occupancy of an acute care bed may leave older adults vulnerable to hospital- associated complications, such as falls, pressure ulcers, functional decline, and delirium (Covinsky, Pierluissi, Johnston, 2011; Halfon, Eggli, Van Melle, \& Vagnair, 2001; Murray, Cameron, \& Cumming, 2007; Rockwood, et al., 2005; Siddiqi, House, \& Holmes, 2006). These adverse events can further contribute to both prolonged hospitalizations, increase readmissions rates, and can decrease the likelihood of seniors returning to an independent or supported state of living in the community (Wong, et al., 2014).

As individuals age, they are more likely to reside in collective dwellings, such as nursing homes, chronic care facilities, and long-term care hospitals (CIHI, 2017). In Canada, $6.8 \%$ of seniors aged 65 and older were residing in nursing homes or long-term residence; this proportion increases to 30\% among older seniors, aged 85 and older (CIHI, 2017). According to the CIHI (2017), as many as one in five seniors who enter residential care following an acute care admission may have been able to be supported in the home. While the cost of providing care for seniors in long-term care is significantly less than providing equivalent care in a hospital setting, it remains the most costly care option for seniors along the continuum of seniors' care (Ontario 
Association of Non-Profit Homes and Services for Seniors [OANHSS], 2016). Therefore, supporting safe transitions back to the community, when attainable, may also reduce the economic burden of long-term facility use.

The majority (92.1\%) of individuals aged 65 and over reside in the community and want to live at home as long as possible (CMA, 2016). Ideally, seniors who are discharged following an acute care admission return to their previous state of living. The CIHI (2011) found that, regardless of the potential complexity of their care needs, nine out of ten seniors with at least one chronic condition have a desire to manage their medical needs at home. To achieve this, seniors may require assistance, including meal and transportation, home health services, and activities to prevent loneliness and isolation (CMA, 2016). The ability to account for these factors in the home may promote senior's overall quality of life, dignity, and autonomy (CIHI, 2011; CMA, 2016).

As exemplified, high-quality coordination of care during the transition from hospital to home may improve the economic burden on acute care systems and promote individual needs. Therefore, the advantages of transition-based interventions can be evaluated based on a variety of quality of care indicators, including health service utilization, health status related outcomes, and/or self-rated objectives (Allen et al., 2014; Burke et al., 2013; Coleman et al., 2003). Health service utilization measures are often categorized using indicators of efficiency and effectiveness, such as hospital length of stay, readmission rates, visits to primary care, and/or use of social services (Allen et al., 2014). Mortality rates, functional/physical status, and the ability to perform activities of daily living, are commonly reported health status outcomes (Allen et al., 2014; Burke et al., 2013; Coleman et al., 2003; Jeffs et al., 2017; Naylor, 2012). Self- rated outcomes of quality often include the following measures: self-management; self- rated 
symptoms; quality of life; patient satisfaction; reductions in medication errors; and the provision of, and access to, safe and timely care (Allen et al., 2014; Burke et al., 2013; Coleman et al., 2003; Jeffs et al., 2017; Naylor, 2012).

Transition-based interventions for seniors need to consider the many factors (increased complexity of care, polypharmacy, informal caregivers, financial deficit, lack of access to timely care) that can contribute to adverse outcomes, decreased patient satisfaction, and increased hospital expenditures, to ensure quality of care. Multiple studies have been conducted to evaluate the effectiveness of transition -based interventions using different measures of quality (Hsaio-mei, Yi-Hsuan, \& Ching-Min, 2017; Kim \& Thyer, 2015; Kirk, 2014; Le Berre, Maimon, Sourial, Guériton, \& Vedel, 2017; Mora, Dorrejo, Carreon, \& Butt, 2017). However, a lack of standardization of the intervention content and inconsistent findings have been observed within the transition-based literature. More specifically, variation in the characteristics of transitionsinterventions, including the component (the type of activities that make up the intervention), mode (the format in which the intervention was delivered), dose (the length of time and frequency), and approach (how the intervention was delivered), have been observed, which may result in erroneous conclusions (Sidani \& Braden, 1998). The potential effectiveness of complex transitional care interventions may depend on a prior knowledge of the most effective characteristics of transition-based interventions (Kansagara et al., 2016; Lowthian, 2017). Further empirical research is required to determine the most effective transitional care intervention characteristics to support the healthcare system and the health and autonomy of seniors who are transitioning from acute care to home. 


\section{Research Problem}

Historically, hospitals were designed to rapidly diagnose as well as to medically or surgically treat an acute illness (Wong, et al., 2014). With the growing demands associated with an aging population, hospital organizations require a shift in design to optimize care for the senior population. As the population ages, one of the primary goals of health care services is to avoid or reduce costs associated with the institutionalization of seniors (CMA, 2016). Policies that focus on seniors' care in Canada aims to promote this goal by supporting the independence of older Canadians in their homes and communities (CMA, 2016). Opportunities to delay or avoid reliance on institutionalized care not only promotes senior's health and autonomy yet may also assist with health resources utilization objectives (CIHI, 2017). To achieve these healthcare goals, acute care services have strived to develop effective transitional care interventions to support the safe and timely transition of seniors from acute care to home.

Several empirical studies and literature reviews have been conducted to evaluate transition interventions aimed to uphold quality of care (Hsaio-mei et al., 2017; Kim \& Thyer, 2015; Kirk, 2014; Le Berre et al., 2017; Mora et al., 2017). Many indicators of quality have been identified in the research related to transitional care-interventions. Empirical research has yielded inconsistent findings, which may be the result of an intervention that was not well defined or described, clearly circumscribed, and/or carefully operationalized (Sidani \& Braden, 1998). A preliminary review of empirical intervention studies suggests that there is significant variation in the intervention characteristics; more specifically, the component, mode, dose, and approach of such interventions are inconsistent amongst studies. The level of complexity, clinical nature, their dose or strength, and the resources needed to implement the intervention 
should be considered when designing, implementing, and evaluating intervention studies (Sidani \& Braden, 1998).

Current review studies describe an association between certain intervention characteristics and outcomes of interests (Hsaio-mei et al., 2017; Kim \& Thyer, 2015; Kirk, 2014; Le Berre et al., 2017; Mora et al., 2017). Researcher's commonly consider the potential influence of the number and type of intervention components (Hsaio-mei et al., 2017; Kim \& Thyer, 2015; Kirk, 2014; Le Berre et al., 2017; Mora et al., 2017), while only a limited number of studies considered intervention dose (Kim \& Thyer, 2015; Kirk, 2014; Le Berre et al., 2017; Mora et al., 2017) and mode (Hsiao-Mei et al., 2017; Le Berre et al., 2017), while evaluating the effectiveness of a given intervention. The implications of the approach of a given intervention (being structured or tailored) has not been considered in terms of outcome measures. Notably, no review study has considered the potential relationship between each intervention characteristic and a statistically significance change in a given quality of care indicator. Therefore, despite the extensive literature on transition interventions, the specific intervention characteristics found to be most effective at promoting quality of care for seniors remains unknown. A systematic review is warranted to comprehensively explore how the component, mode, dose, and approach of transitional care interventions may be associated with quality of care during transitions.

\section{Purpose Statement}

The purpose of this systematic review was to identify the characteristics of transitionbased interventions that have been effective at enhancing quality of care for seniors being discharged home from acute care. More specifically, the component, mode, dose, and approach of interventions associated with a statistically significant change across a variety of quality of 
care indicators were examined. These indicators included health service outcomes (e.g.

readmission rates), health status outcomes (e.g. mortality rate), and self-rated outcomes (e.g. quality of life).

\section{Significance}

A comprehensive understanding of each characteristic will support the development and implementation of effective transitional care interventions, while supporting the provision of evidence-informed practice. More specifically, as interventions are used to prevent, resolve, or manage health-related problems, a greater understanding of the most effective characteristics may result in financial and individual benefits (Sidani \& Braden, 1998). Sidani and Braden (1998) describe the ultimate outcome of intervention-based research to be the absence of the undesired status. As an ineffective transitional care plan of care for seniors may result in negative individual and system outcomes, any intervention that aims to resolve this issue is treating both the underlying problem and its systemic effects.

A thorough examination of the nature of complex transitional care interventions may have research-based benefits. Awareness of the most effective component, mode of delivery, amount, frequency, duration, and approach of interventions will assist in designing and evaluating future transition-based interventions. For example, interventions could be designed that incorporate the number and type of components recommended in this review. Lastly, this systematic review will provide an enhanced understanding of the ideal type of transition-based intervention that would be effective in enhancing quality of care among seniors. 


\section{Chapter Two}

\section{Empirical Literature Review}

The following chapter provides an overview of the current state of knowledge regarding interventions designed to support the transition from acute care to home for seniors. Secondary sources of data, including literature and systematic reviews that examined intervention characteristics, were selected. The intervention characteristics reviewed included the component (number, type of elements in intervention), approach (tailored, standardized, or combination), mode (strategy, format of delivery), and dose (amount, frequency, duration) across studies (Sidani \& Braden, 1998). This review included a critique of the existing literature and reported on limitations and gaps in the literature. This section concludes with a discussion of the potential contribution to gaps in knowledge this systematic review will have.

\section{Search Criteria}

The systematic search for relevant literature was performed using the main healthcare electronic databases, including the Cumulative Index to Nursing and Allied Health (CINAHL), Medline, Cochrane Library, and Proquest. The following search terms guided the review: aged (65-79), aged 80 and over, acute care, hospitalized, nursing care, continuity of care, transitional care, intervention, and intervention characteristics (component, mode, dose and approach) (Table 1). The Boolean operators AND and OR were used. The search was limited to articles written in English, with no specific publication date being identified to ensure all relevant studies are identified. The search was conducted with the assistance of a university librarian.

Empirical studies were deemed relevant for this review based on the following criteria: (1) written in the English language; (2) the sample consisted of senior patients (65 years or 
older); (3) the study focused on seniors' transitioning from an acute care inpatient ward to home; (4) the article reviewed any/all intervention characteristics (including component, mode, dose, or approach) related to transitions of care for seniors from acute care to home and (5) the study was a systematic review, review of the literature, and/or meta-analysis.

Empirical studies were excluded from this review based on the following exclusion criteria: (1) the study did not report one or more of the domains of transitional care; (2) the study was not a peer-reviewed report; (3) the population of interest did not include seniors and/or caregivers (health care provider was the population of interest). The exclusion criteria were kept broad to ensure that all studies examining the characteristics of transition-based interventions were identified.

\section{Search Strategy}

Tables 1-3 display the search terms, provided using PICO format, and the search strategy used for each database. Table 1 describes the search strategies used for the CINAHL database. A similar strategy was applied to Medline, shown in Table 2. The COCHRANE database required a broader search strategy, using similar key terms, which is described in Table 3. Lastly, the search strategy was applied to the Proquest database, shown in table 4. In addition to the search terms described using PICO, the following additional limits were placed based on the inclusion and exclusion criteria set forth for this review: date (2008-2018); age group (65+ years), scholarly journal, peer reviewed, English language, and review study.

\section{Table 1: CINAHL}

\begin{tabular}{|l|l|l|l|l|}
\hline Aim & Search term(s) & $\begin{array}{l}\text { Number of } \\
\text { results }\end{array}$ & $\begin{array}{l}\text { Number of } \\
\text { matches }\end{array}$ \\
\hline
\end{tabular}




\begin{tabular}{|c|c|c|c|c|}
\hline Population (P) & $\begin{array}{l}\text { Individuals above } 65 \\
\text { years. }\end{array}$ & $\begin{array}{l}\text { "aged", "aged, } 80 \\
\text { and over" }\end{array}$ & & \\
\hline Intervention (I) & $\begin{array}{l}\text { Transitions of care } \\
\text { from acute care to } \\
\text { home. }\end{array}$ & $\begin{array}{l}\text { "transitional } \\
\text { care", "nursing } \\
\text { intervention", } \\
\text { "continuity of } \\
\text { patient care", } \\
\text { "intervention } \\
\text { characteristics" }\end{array}$ & & \\
\hline Comparison (C) & Usual care. & & & \\
\hline Outcome $(\mathrm{O})$ & Quality of care. & & & \\
\hline Other Criteria & Methods: review. & $\begin{array}{l}\text { "Systematic } \\
\text { review", "review", } \\
\text { "literature review" }\end{array}$ & & \\
\hline $\mathrm{P}+\mathrm{I}+\mathrm{C}+\mathrm{O}$ & & $\begin{array}{l}\text { "Systematic } \\
\text { review", AND } \\
\text { "transitional } \\
\text { care", AND } \\
\text { "aged", OR "aged, } \\
80 \text { and over" }\end{array}$ & 10 & 2 \\
\hline $\mathrm{P}+\mathrm{I}+\mathrm{C}+\mathrm{O}$ & & $\begin{array}{l}\text { "Transitional } \\
\text { care", AND } \\
\text { "aged" OR "aged } \\
80 \text { and over", } \\
\text { AND "nursing } \\
\text { intervention" }\end{array}$ & 2 & 0 \\
\hline $\mathrm{P}+\mathrm{I}+\mathrm{C}+\mathrm{O}$ & & $\begin{array}{l}\text { "literature } \\
\text { review", AND } \\
\text { "aged" OR "aged, } \\
80 \text { and over", } \\
\text { AND "transitional } \\
\text { care" }\end{array}$ & 1 & 0 \\
\hline $\mathrm{P}+\mathrm{I}+\mathrm{C}+\mathrm{O}$ & & $\begin{array}{l}\text { "Transitional } \\
\text { care", AND } \\
\text { "aged" OR "aged } \\
80 \text { and over", } \\
\text { AND } \\
\text { "intervention } \\
\text { characteristics" }\end{array}$ & 0 & 0 \\
\hline $\mathrm{P}+\mathrm{I}+\mathrm{C}+\mathrm{O}$ & & $\begin{array}{l}\text { "continuity of } \\
\text { patient care", } \\
\text { AND "aged" OR } \\
\text { "aged } 80 \text { and } \\
\text { over", AND } \\
\text { "nursing } \\
\text { intervention" OR }\end{array}$ & 4 & 1 \\
\hline
\end{tabular}




\begin{tabular}{|l|l|l|l|}
\hline & $\begin{array}{l}\text { "intervention", } \\
\text { AND “literature } \\
\text { review" OR } \\
\text { "systematic } \\
\text { review" }\end{array}$ & & \\
\hline
\end{tabular}

Table 2: Medline

\begin{tabular}{|c|c|c|c|c|}
\hline & Aim & Search term(s) & $\begin{array}{l}\text { Number of } \\
\text { results }\end{array}$ & $\begin{array}{l}\text { Number of } \\
\text { matches }\end{array}$ \\
\hline Population $(\mathrm{P})$ & $\begin{array}{l}\text { Individuals above } 65 \\
\text { years. }\end{array}$ & $\begin{array}{l}\text { "aged", "aged, } 80 \\
\text { and over" }\end{array}$ & & \\
\hline Intervention (I) & $\begin{array}{l}\text { Transitions of care } \\
\text { from acute care to } \\
\text { home. }\end{array}$ & $\begin{array}{l}\text { "transitional } \\
\text { care", "continuity } \\
\text { of patient care", } \\
\text { "intervention } \\
\text { characteristics" }\end{array}$ & & \\
\hline Comparison (C) & Usual care. & & & \\
\hline Outcome $(\mathrm{O})$ & Quality of care. & & & \\
\hline Other Criteria & Methods: review. & $\begin{array}{l}\text { "Systematic } \\
\text { review", } \\
\text { "literature review" }\end{array}$ & & \\
\hline $\mathrm{P}+\mathrm{I}+\mathrm{C}+\mathrm{O}$ & & $\begin{array}{l}\text { "Systematic } \\
\text { review", AND } \\
\text { "transitional } \\
\text { care", AND } \\
\text { "aged", AND } \\
\text { "aged, } 80 \text { and } \\
\text { over" }\end{array}$ & 5 & 1 \\
\hline $\mathrm{P}+\mathrm{I}+\mathrm{C}+\mathrm{O}$ & & $\begin{array}{l}\text { "literature } \\
\text { review", AND } \\
\text { "aged" AND } \\
\text { "aged, } 80 \text { and } \\
\text { over", AND } \\
\text { "transitional care" }\end{array}$ & 2 & 0 \\
\hline $\mathrm{P}+\mathrm{I}+\mathrm{C}+\mathrm{O}$ & & $\begin{array}{l}\text { "Transitional } \\
\text { care", AND } \\
\text { "aged" AND } \\
\text { "aged } 80 \text { and } \\
\text { over", AND } \\
\text { "intervention } \\
\text { characteristics" }\end{array}$ & 1 & 0 \\
\hline
\end{tabular}




\begin{tabular}{|c|c|c|c|}
\hline $\mathrm{P}+\mathrm{I}+\mathrm{C}+\mathrm{O}$ & $\begin{array}{l}\text { "continuity of } \\
\text { patient care", } \\
\text { AND "aged" } \\
\text { AND "aged } 80 \\
\text { and over", AND } \\
\text { "nursing } \\
\text { intervention" OR } \\
\text { "intervention", } \\
\text { "literature review" } \\
\text { OR "systematic } \\
\text { review" }\end{array}$ & 28 & 1 \\
\hline
\end{tabular}

Table 3: COCHRANE

\begin{tabular}{|c|c|c|c|c|}
\hline & Aim & Search term(s) & $\begin{array}{l}\text { Number of } \\
\text { results }\end{array}$ & $\begin{array}{l}\text { Number of } \\
\text { matches }\end{array}$ \\
\hline Population $(\mathrm{P})$ & $\begin{array}{l}\text { Individuals above } 65 \\
\text { years. }\end{array}$ & "aged" & & \\
\hline Intervention (I) & $\begin{array}{l}\text { Transitions of care } \\
\text { from acute care to } \\
\text { home. }\end{array}$ & $\begin{array}{l}\text { "transitions of } \\
\text { care", "continuity } \\
\text { of patient care", } \\
\text { "intervention", }\end{array}$ & & \\
\hline Comparison $(\mathrm{C})$ & Usual care. & & & \\
\hline Outcome (O) & Quality of care. & & & \\
\hline Other Criteria & Methods: review. & & & \\
\hline $\mathrm{P}+\mathrm{I}+\mathrm{C}+\mathrm{O}$ & & $\begin{array}{l}\text { "transitions of } \\
\text { care", AND } \\
\text { "aged" }\end{array}$ & 1 & 0 \\
\hline $\mathrm{P}+\mathrm{I}+\mathrm{C}+\mathrm{O}$ & & $\begin{array}{l}\text { "Transitional } \\
\text { care", AND } \\
\text { "intervention" }\end{array}$ & 8 & 0 \\
\hline $\mathrm{P}+\mathrm{I}+\mathrm{C}+\mathrm{O}$ & & $\begin{array}{l}\text { "continuity of } \\
\text { patient care", } \\
\text { AND "aged" }\end{array}$ & 39 & 0 \\
\hline
\end{tabular}

Table 4: Proquest 


\begin{tabular}{|c|c|c|c|c|}
\hline & Aim & Search term(s) & $\begin{array}{l}\text { Number of } \\
\text { results }\end{array}$ & $\begin{array}{l}\text { Number of } \\
\text { matches }\end{array}$ \\
\hline Population $(\mathrm{P})$ & $\begin{array}{l}\text { Individuals above } 65 \\
\text { years. }\end{array}$ & $\begin{array}{l}\text { "aged", "aged, } 80 \\
\text { and over" }\end{array}$ & & \\
\hline Intervention (I) & $\begin{array}{l}\text { Transitions of care } \\
\text { from acute care to } \\
\text { home. }\end{array}$ & $\begin{array}{l}\text { "transitional } \\
\text { care", "nursing } \\
\text { intervention", } \\
\text { "continuity of } \\
\text { patient care", } \\
\text { "intervention } \\
\text { characteristics" }\end{array}$ & & \\
\hline Comparison (C) & Usual care. & & & \\
\hline Outcome (O) & Quality of care. & & & \\
\hline Other Criteria & Methods: review. & $\begin{array}{l}\text { "Systematic } \\
\text { review", "review", } \\
\text { "literature review" }\end{array}$ & & \\
\hline $\mathrm{P}+\mathrm{I}+\mathrm{C}+\mathrm{O}$ & & $\begin{array}{l}\text { "Systematic } \\
\text { review", } \\
\text { "Transitional } \\
\text { care", AND } \\
\text { "aged" }\end{array}$ & 6 & 0 \\
\hline $\mathrm{P}+\mathrm{I}+\mathrm{C}+\mathrm{O}$ & & $\begin{array}{l}\text { "systematic } \\
\text { review", } \\
\text { "Transitional } \\
\text { care", AND } \\
\text { "aged" OR "aged } \\
80 \text { and over", } \\
\text { AND } \\
\text { "intervention } \\
\text { characteristics" }\end{array}$ & 6 & 0 \\
\hline $\mathrm{P}+\mathrm{I}+\mathrm{C}+\mathrm{O}$ & & $\begin{array}{l}\text { "continuity of } \\
\text { care OR discharge } \\
\text { planning OR } \\
\text { transitional care", } \\
\text { AND "review" }\end{array}$ & 33 & 0 \\
\hline
\end{tabular}

Using this approach, the electronic search yielded 146 articles, of which 125 were excluded after screening for relevancy as they did not meet in inclusion criteria for this review. Initially, 21 studies appeared relevant based on the title and abstract; these studies were located and reviewed thoroughly. Of these 21 articles, the main reasons for exclusion were ineligible 
study design $(n=3)$, study population $(n=5)$, or transition- destination (e.g. nursing home) $(n=6)$, or more than one of the aforementioned reasons $(n=2)$. As a result of the comprehensive literature search, five articles were deemed relevant for this review (Hsiao-Mei et al., 2017; Kim \& Thyer, 2015; Kirk, 2014; Le Berre et al., 2017; Mora et al., 2017). See Appendix A for a table summarizing the included articles.

\section{Characteristics of Included Reviews}

Of the five articles, one used a systematic review design (Le Berre et al., 2017), two were literature reviews (Kim \& Thyer, 2015; Kirk, 2014) one was a meta-analytic study (Hsiao-Mei et al., 2017), and one was an integrative review (Mora et al., 2017). Most of the articles $(n=3)$ limited their sample to randomized controlled trials (RCTs) (Hsiao-Mei et al., 2017; Kim \& Thyer, 2015; Le Berre et al., 2017). The review conducted by Mora et al. (2017) included primary research that used a variety of research design methodologies, including three RCTs, one meta-analysis, and four non-randomized studies. Kirk (2014) included RCTs and quasiexperimental research studies in their sample. Each study limited the sample population to senior patients (65 years or older) transitioning from acute care to home.

Four of the included reviews examined multiple domains of transitional care, ranging from hospital-based interventions to home care interventions (Hsiao-Mei et al., 2017; Kim \& Thyer, 2015; Le Berre et al., 2017; Mora et al., 2017), while one review evaluated the effectiveness of a single transitional care strategy (telephone follow-up) (Kirk, 2014). Additionally, most of the reviews reported that a range of healthcare providers (physicians, nurses, social workers, physical therapists) may provide the transition-based intervention; however, one review (Mora et al., 2017) limited their study to nurse-practitioner led transitional care interventions. 
Quality of care measures varied considerably among the five included articles, ranging from healthcare utilization, health status, and self-rated outcomes. Of the five articles, two (Le Berre et al., 2017; Mora et al., 2017) used each outcome type, including measures of health care utilization (e.g. readmission rates, emergency visits), health status (e.g. mortality and functional status), and self-rated outcome measures (e.g. quality of life, patient satisfaction). Two reviews were limited to health care utilization outcomes (Kim \& Thyer, 2015; Kirk, 2014), while one was limited to self-rated measures, evaluating aspects of quality of life (Hsiao-Mei et al., 2017).

\section{Intervention Characteristics: Component, Mode, Dose, Approach}

Intervention components are the activities the intervention is comprised of that are directed toward reaching a common objective (Sidani \& Braden, 1998). Researchers classified studies based on the intervention components, including hospital-based interventions and/or home care interventions. The number and type of components were reported across studies and evaluated based on their ability to produce the desired outcome. Educational components were reported in four reviews (Hsaio-Mei et al., 2017; Kim \& Thyer, 2015; Le Berre et al., 2017; Mora et al., 2017) and included the following activities: intensive individualized patient education, self-management, and detailed discharge treatment summary. Medication management components were included in four reviews (Hsaio-Mei et al., 2017; Kim \& Thyer, 2015; Le Berre et al., 2017; Mora et al., 2017) and included medication-related reconciliation, consultation, and counseling. Supportive care components, including continued care in the community, follow-up care, and coordination/collaboration between inpatient and outpatient healthcare providers, were reported across the five reviews. Lastly, organizational components, including rehabilitation programs, standardized discharge planning summaries, early discharge 
planning, and multidisciplinary coordination, were included in three reviews (Hsaio-Mei et al., 2017; Kim \& Thyer, 2015; Mora et al., 2017).

The dose of interventions, defined as the strength of an intervention (amount, frequency and duration), was considered, in part, in each study. The duration of the intervention was the most commonly reported aspect of dose (n=5) (Hsaio-Mei et al., 2017; Kim \& Thyer, 2015; Kirk, 2014; Le Berre et al., 2017; Mora et al., 2017). Other dimension of dose, including amount and frequency, were under-reported in most reviews. Research by Mora et al. (2017) was the only review to report on the differences between the amount of intervention the participants were exposed to and its potential impact on the intervention effectiveness. Studies by Kirk (2014) and Le Berre et al. (2017) were the only articles to report on multiple dimensions (frequency and duration) of the intervention dose. Notably, for articles that reported on one or more dimension of the dose of interventions, the range between the amount, frequency and duration of the interventions varied considerably.

The mode of the intervention reflects the medium (means through which the intervention is given), and format (the specific technique used to provide the intervention) (Sidani, 2015; Sidani \& Braden, 2011). In terms of mode, most studies included some details pertaining to the intervention's mode of delivery. Aspects relating to the medium of delivery, including face-toface home visits and/or follow-up telephone calls, were reported in all reviews (Hsiao-Mei et al., 2017; Kim \& Thyer, 2015; Kirk, 2014; Le Berre et al., 2017; Mora et al., 2017). Information regarding other elements of transitional care, such as the mode of delivery of patient education and discharge summaries, were not commonly described.

The format of the information, that is, who provided the intervention (e.g. registered nurses, social workers, physicians) and who the intervention was provided to (e.g. senior, senior 
and caregiver) were reported in all reviews (Hsiao-Mei et al., 2017; Kim \& Thyer, 2015; Kirk, 2014; Le Berre et al., 2017; Mora et al., 2017). However, only a limited number of reviews ( $\mathrm{n}=$ 2) considered the potential impact the format of the intervention may have on outcomes of interest (Hsiao-Mei et al., 2017; Le Berre et al., 2017).

The approach of an intervention refers to the structure in which the intervention was given, being standardized or tailored in nature. No reviews commented on details relating to the approach of the interventions.

\section{Research Findings}

The reviews synthesized a large variation of interventions that ranged from no improvements to statistically significant improvements (Hsaio-mei et al., 2017; Kim \& Thyer, 2015; Kirk, 2014; Le Berre et al., 2017; Mora et al., 2017). The reviews varied considerably in the description of intervention characteristics and their perceived influence on outcome measures. More specifically, most reviews considered the potential influence of the number and type of intervention components included (Hsaio-mei et al., 2017; Kim \& Thyer, 2015; Kirk, 2014; Le Berre et al., 2017; Mora et al., 2017), while only a limited number considered intervention dose (Kim \& Thyer, 2015; Kirk, 2014; Le Berre et al., 2017; Mora et al., 2017) and mode (Hsiao-Mei et al., 2017; Kirk, 2014; Le Berre et al., 2017) while evaluating the effectiveness of a given intervention. The approach of the intervention, being structured or tailored, was not considered in terms of outcome measures.

Each of the reviews described a relationship between specific intervention characteristics and outcomes of interest (Hsaio-mei et al., 2017; Kim \& Thyer, 2015; Kirk, 2014; Le Berre et al., 2017; Mora et al., 2017). In terms of component, articles that examined single and 
multicomponent interventions (Hsaio-mei et al., 2017; Kim \& Thyer, 2015; Kirk, 2014; Le Berre et al., 2017; Mora et al., 2017) reported greater results when multiple intervention components were used. The review by Hsaio-mei et al. (2017) synthesized seven studies that showed improvements in physical and mental health measures of quality of life when combining the following intervention characteristics: home visits or telephone consultations, detailed treatment summary, and caregiver resources. Kirk's (2014) review, which examined telephone follow-up, reported more effective results when used in combination with different elements of transitional care; telephone follow-up used in combination with discharge planning, education, and home visits were found most effective in producing the desired effects. Both reviews reported no significant improvement in outcome measures in interventions that included only a single component, such as supportive care strategies (follow-up in the home) (Hsaio-mei et al., 2017; Kirk, 2014).

In terms of dose, two reviews reported on the most effective duration of treatment interventions (Mora et al., 2017). The review conducted by Mora et al. (2017) concluded that interventions which followed patients for at least two months were most successful in reducing readmissions; Le Berre et al. (2017) concluded interventions must include follow-up for a minimum of six months to improve measures of quality of life.

Notably, both Kim and Thyer (2015) and Kirk (2014) compared three transition-based interventions (Naylor et al., 1994; Naylor et al., 1999; Naylor et al., 2004) which may highlight the importance of considering the characteristics of transition-based interventions. Throughout the progression of their research, Naylor and colleagues increased the number of transitional care components (adding supportive care elements such as home visits in the latter trial) and the length of time in which the treatment intervention was implemented (from 6 weeks in the earlier 
trial to 12 months in the latter) (Naylor et al., 1994; Naylor et al., 1999; Naylor et al., 2004); compared to their earlier intervention (Naylor et al., 1994), the intervention in the Naylor et al. (2004) study produced improvements in a variety outcomes measures, including reduced healthcare costs, improved health, and improved patient satisfaction. Overall, the number and type of components and the dose had a direct impact on intervention effectiveness.

The most effective medium of delivery of interventions was considered in two reviews (Kirk, 2014; Mora et al., 2017). Research by Mora et al. (2017) reported interventions that included nurse practitioner- led follow-up phone calls and in-person home visits were more successful at reducing readmissions (however were not statistically significant). Additionally, Kirk (2014) concluded that using telephone contact as the sole method of follow-up in transitional care may be ineffective in reducing health service utilization measures such as readmission rates; alternatively, telephone follow-up used in combination with in-person home visits may be more effective.

Reviews by Hsiao-Mei et al. (2017) and Le Berre et al. (2017) considered the format of the intervention in their evaluation process. According to Le Berre et al. (2017), interventions lead by a nurse did not lead to improved results in a variety of outcome measures, whereas, involvement of a pharmacist may result in improved outcomes. In contrast, Hsiao- Mei et al. (2017) determined that interventions lead by nurses, physicians, nutritionists, social workers, or physical therapists can improve both physical and mental components of quality of life.

\section{Critique of Empirical Evidence}

Following a thorough examination of these reviews, several limitations were identified that included a lack of standardization of the intervention characteristics. Reviews varied in 
terms of the number and type of components included in each intervention, the dose (amount, frequency, duration), mode of delivery (medium, format), and approach (standardized or tailored) to interventions. Such variation in the intervention characteristics may influence the achievement of the desired outcomes (Sidani \& Braden, 2011). Interventions designed without taking the diverse characteristics into consideration may result in erroneous conclusions regarding the intervention effectiveness (Sidani \& Braden, 1998). The variance in intervention design may be a direct result of an inadequate understanding of the most effective characteristics, specifically the component, approach, mode, and dose, associated with the achievement of desired effects.

Many reviews have described or summarized the intervention characteristics of primary research studies within their review (Hsaio-mei et al., 2017; Kim \& Thyer, 2015; Kirk, 2014; Le Berre et al., 2017; Mora et al., 2017). However, few reviews have thoroughly explored the relationship between intervention characteristics and quality of care during their evaluation process. More specifically, no review has considered the potential impact of each intervention characteristic, including component, mode, dose and approach, on the effectiveness of a given intervention. As factors related to each intervention characteristic may have a direct effect on the integrity of intervention implementation, further research is warranted (Sidani \& Braden, 1998).

Lastly, the reviewed literature largely focused on one outcome of interest (Hsaio-Mei et al., 2017; Kim \& Thyre, 2015), often limiting outcomes to either health service utilization measures (Kim \& Thyre, 2015; Kirk, 2014) or self-rated outcome measures (Hsaio-Mei et al., 2017). Only two reviews examined a combination of the three outcomes measures, including health service utilization, health status, and self-rated outcomes (Le Berre et al., 2017; Mora et al., 2017). No review has thoroughly considered the effect of intervention characteristics on the 
broad range of quality of care indicators that exist in the transitional care literature when evaluating a given intervention.

Successful implementation of complex, multicomponent interventions requires knowledge of the most effective activities forming each component, its mode of delivery, its approach, and its required dose (Sidani \& Braden, 1998). The success of future transition-based interventions may be contingent on the availability of this information. Therefore, to address the current research gap, this review reports on the influence intervention characteristics may have on the achievement of desired treatment outcomes. Interventions were described and evaluated based on their number and type of components, dose, mode, and approach of delivery. Intervention characteristics were evaluated on their effectiveness in producing a variety of quality of care outcome measurements, including health service utilization, health status, and self- rated outcomes. 


\section{Chapter Three}

\section{Conceptual Framework}

This chapter provides conceptual and operational definitions of the key variables of interest. The following terms were defined: transitional care intervention, intervention characteristics (component, mode, dose and approach), and quality of care. Key variables were discussed and explored through the lens of transitional care for seniors from acute care to home. A relational statement was provided to explicate relationships among study variables based on the findings from the literature review. Lastly, research questions were proposed based on the findings from the empirical literature review and conceptual framework.

\section{Transitional Care Interventions}

\section{Conceptual Definition of Transition Care Interventions.}

Interventions refer to treatments, therapies, procedures, or actions which healthcare providers implement to or with clients to help them move towards more optimal health outcomes (Sidani \& Braden, 1998). More specifically, interventions are actions given in response to an identified problem (Sidani \& Braden, 2011). Depending on the nature of the problem, interventions may be given to, on behalf of, or with clients, (Sidani \& Braden, 2011). Actions may be implemented independently or in collaboration with other members of the multidisciplinary team. Clients (those requiring the intervention) can include individuals, families, or communities (Sidani \& Braden, 2011).

Interventions are often characterized by the identified preset goal, the type of actions comprising them, and the level of complexity (Sidani \& Braden, 2011). The goal of an intervention can be designed to either prevent or manage healthcare issues (Sidani \& Braden, 2011). Intervention actions are determined by the nature of the presenting problem and can be physical, behavioural, psychological, or social in nature (Sidani \& Braden, 2011). The level of 
complexity relates to the number of components and the required actions within each component of the intervention (Sidani \& Braden, 2011). Interventions are considered complex if they consist of multiple interrelated and/or interdependent components (Blackwood, 2005).

Transitional care interventions have been designed and implemented to support the coordination of seniors as they transfer between different locations such as an acute care setting to home (Coleman et al., 2003; Kirk, 2014). Transitional care interventions are intended to ensure continuity of healthcare, avoid adverse outcomes, and promote the safe and timely transfer of clients between settings or levels of care (Naylor, 2012). Naylor (2012) asserts that effective transitional care must consider complex care populations, such as the senior population, that are often vulnerable to poor outcomes during healthcare transitions. Transition-based interventions may include actions both pre-and-post-discharge and often include multiple components, such as discharge planning and outpatient referrals, making them complex in nature (Burke et al., 2014; Sidani \& Braden, 2011).

Transitional care is a multidimensional concept that comprises of several continuity of care elements (Kirk, 2014; Le Berre et al., 2017). Burke et al. (2014) identifies a range of care activities involved in quality transitions, consisting of: (1) discharge planning, (2) complete communication of information, (3) availability, timeliness, clarity, and organization of information, (4) medication safety, (5) educating patient to promote self-management, (6) enlisting help from social and community supports, (7) advance care planning, (8) coordinating care among team members, (9) monitoring and managing symptoms after discharge, (10) outpatient follow-up. The validated framework provides a means of categorizing the many transitional care interventions (Burke et al., 2014; Burke et al., 2013). 
Naylor et al. (2011) implemented a transitional care program exclusively led by advance practice nurses using similar components. Their research included multicomponent transitionbased programs which considered continuity of care for patients transitioning across settings and included the following components: hospital and home visits, patient education, symptom management, communication among patients, caregivers, and providers, and coordination of care (Mora et al., 2017; Naylor et al., 2011).

A variety of health care professionals, including registered nurses, advance practice nurses, social workers, physicians, pharmacists, and/or physiotherapist/occupational therapists, may be involved in the implementation of transitional care interventions by (Mora et al., 2017). Furthermore, many interventions require a multidisciplinary approach to interventionimplementation.

\section{Operational Definition of Transition Interventions.}

For the purposes of this study, transition interventions were operationalized by the domain of transitional care the intervention aims to address. Domains of transitional care included any intervention that addressed one or more of the following activities: discharge planning, complete communication of information, availability, timeliness, clarity, and organization of information, medication safety, educating patient to promote self-management, enlisting help from social and community supports, advance care planning, coordinating care among team members, monitoring and managing symptoms after discharge, outpatient follow-up (Burke et al., 2013). As transition interventions are often complex in nature, this review also identified whether the intervention addresses multiple domains of transitional care.

\section{Intervention Characteristics}


In this systematic review, Sidani's intervention characteristics (1998), including component, mode, dose and approach, were used. Conceptual and operational definitions for each characteristic are found below.

\section{Component.}

Conceptual Definition of Component. A component is defined as a set of interconnected activities that address one aspect of the healthcare problem or that address a particular domain of the clients' healthcare condition (Sidani, 2015). The number of components can vary between interventions; simple interventions involve a single component, while complex interventions comprise of multiple components (Sidani \& Braden, 2011). Structuring the intervention into components may ensure that all aspects of the preset problem and/or its manifestations or determinants are targeted (Sidani \& Braden, 2011). Within the realm of complex healthcare interventions, components are defined as the parts of the complex intervention that are distinct, yet, contribute to the whole of the intervention in full or part (Clark, 2013).

The specific strategies that characterize transition interventions have been theoretically hypothesized and empirically tested by several researchers (Hsaio-Mei et al., 2017; Kim \& Thyer, 2015; Kirk, 2014; Le Berre et al., 2017; Mora et al.,2017; Sidani \& Braden, 2011). Research by Naylor (2000) identified five components of transitional care: (1) communication of information; (2) patient education; (3) enlisting the help of social and community supports; (4) ensuring supportive care; and (5) coordinating care among team members.

Burke et al. (2013) categorized the intervention strategies into the following domains: (1) discharge planning; (2) complete communication of information; (3) availability, timeliness, clarity, and organization of information; (4) medication safety; (5) educating patient to promote 
self-management; (6) enlisting help from social and community supports; (7) advance care planning; (8) coordinating care among team members; (9) monitoring and managing symptoms after discharge; and (10) outpatient follow-up.

Lastly, Coleman et al. (2003) describes four components of effective transition interventions: (1) communication between providers about the discharge assessment and plan of care; (2) preparation of the patient and carer for the care transition; (3) reconciliation of medications at transition (4) a plan for follow-up and patient education about self-management.

Based on the identified strategies and the findings of the literature review, transitioninterventions for seniors often include educational, supportive care, medication management, and/or organizational components (Burke et al., 2013; Coleman et al., 2003; Hsaio-Mei et al., 2017; Kim \& Thyer, 2015; Kirk, 2014; Le Berre et al., 2017; Mora et al.,2017; Naylor, 2000). As transition interventions for seniors are often complex, a combination of these components may also be applied. Educational components within the transition-literature may include intensive individualized patient education, self-management strategies, and/or detailed discharge summaries (Hsaio-Mei et al., 2017; Kim \& Thyer, 2015; Le Berre et al., 2017; Mora et al., 2017). Supportive care components may include aspects such as enlisting help from social and community supports and preparing the senior and/or their caregiver for care at home; intervention strategies may include home visits, clinic visits, telephone follow-up, and coordination/collaboration between inpatient and outpatient healthcare providers (Hsaio-Mei et al., 2017; Kim \& Thyer, 2015; Kirk, 2014; Le Berre et al., 2017; Mora et al., 2017). Organizational components may include treatments provided in the hospital setting which address healthcare provider activities, and/or the promotion of safe and efficient transitional care. Such components may include rehabilitation programs, discharge planning summaries, early 
discharge planning and multidisciplinary coordination (Hsaio-Mei et al., 2017; Kim \& Thyer, 2015; Mora et al., 2017). Lastly, medication management components are widely incorporated in the transition-based literature (Hsaio-Mei et al., 2017; Kim \& Thyer, 2015; Mora et al., 2017) and include medication-related reconciliation, verification, clarification, and consultation.

Operation Definition of Component. Component was operationalized by whether educational, supportive care, organizational, medication management, or a combination of these components were used. Interventions that included an educational component were operationalized as any intervention that includes information, resources, and/or an education aspect. Supportive care components constitute any intervention that included social, physical, and emotional support and/or resources for the client, family, and/or caregiver throughout the transition from acute care to home. Organizational components were operationalized as any intervention that addressed healthcare provider activities and/or which supported the provision of effective, efficient, equitable, timely, safe and patient-centred transitional care. Medication management components were operationalized as any intervention that provided medication reconciliation, verification, and/or clarification (Pincus, 2013). Interventions which encompassed the collection of medication history, assessment of whether current medications are appropriate, the provision of information to the patient regarding their medication, and/or documentation of changes to medication list, were described as incorporating a medication management component (Pincus, 2013). Interventions that included a combination of these components were operationalized as any intervention that consisted of two or more of these components. Please refer to Appendix I for a breakdown of the component types and examples of each from the transitional care literature.

\section{Mode.}


Conceptual Definition of Mode. Mode of delivery reflects the medium (means through which the intervention is given), and format (the specific technique used to provide the intervention) (Sidani, 2015; Sidani \& Braden, 2011).

The means through which the intervention activities are carried out (medium) may include written and/or verbal strategies. The written medium may include pamphlet, poster, or computer-based formats; the verbal medium may include face-to-face meetings, telephone meetings, and audio/video tapes (Sidani \& Braden, 2011).

The format of transition-based interventions may include how the intervention was provided to the senior and who provided the intervention. Programs may be delivered by a variety of health care workings, including (but not limited to) registered nurses, social workers, physicians, advance practice nurses and/or a multidisciplinary approach (Mora et al., 2017). The format of transition-based interventions also depicts the means through which the intervention is provided, such as individually (to the senior alone), to the senior and their caregiver, or a combination of both.

Operational Definition of Mode. Mode was operationally defined by the medium (means through which the intervention was given) and the format (the specific technique used to provide the intervention). The medium was operationalized by whether the intervention strategies included written and/or verbal techniques, such as face-to-face meetings, telephone sessions, and/or computer-based strategies. The format of the intervention was operationalized based on who provided the intervention (i.e., registered nurses, social workers, physicians, advance practice nurses) and who the intervention was provided to (senior, senior and caregiver etc.). 


\section{Dose.}

Conceptual Definition of Dose. Dose was conceptually defined as the level at which the intervention must be given in order to achieve the desired effect (Sidani, 2015). The intervention dose reflects the amount, frequency and duration of the client's exposure to the intervention (Sidani \& Braden, 2011). The strength (or dose) of the treatment must have the correct intensity and duration to achieve the desired health outcome. Knowledge of the necessary dose information prior to intervention design and implementation may produce more effective interventional research (Sidani \& Braden, 1998).

Heterogeneity may be present within the dose schedules of transition-interventions. Dose schedules may be fixed (the schedule is constant during the study) or variable (the dose schedule may change throughout the study) (Voils et al., 2012). Additionally, dose schedules may be tailored, that is, given in accordance to the senior's needs, or untailored, in which every senior involved in the study receives the same treatment dose (Voils et al., 2012).

Dose was considered for components that involved human contact, such as home visits telephone follow-up, and education sessions. Dose was not considered for transitional care components that involved minimal human contact (i.e. provision of written educational materials) as dose parameters for these intervention components would be difficult to determine (Voils et al., 2012).

Operational Definition of Dose. Dose was operationalized using three indicators: (1) amount; (2) frequency; and (3) duration (Sidani \& Braden, 2011). Amount refers to the number and length of each intervention session (in minutes) (Sidani \& Braden, 2011). Frequency refers to the number of times the intervention session is to be given over a specified time period 
(number of times per month) (Sidani \& Braden, 2011). Lastly, duration is the total length of time the intervention is to be given (in months) (Sidani \& Braden, 2011). Dose was further operationalized by whether a fixed or variable, and tailored or untailored, schedule was used.

\section{Approach.}

Conceptual Definition of Approach. Approach refers to the structure in which the intervention is given; the approach of the intervention may be standardized or tailored in nature (Sidani \& Braden, 2011).

In the standardized approach, the same intervention is delivered to each client, using the same dose (Sidani, 2015). In this type of intervention, the treatment is delivered in a standardized manner and does not consider aspects such as the participant's characteristics, health status, or personal experiences. A standardized transitional care may include discharge summaries, medication review forms, and standardized telephone follow-up questions. In contrast, tailored interventions incorporate individualized interventions that may vary in the mode of delivery and dose used; variance is based on the client's characteristics, needs, and preferences (Sidani, 2015).

The empirical literature review did not report on the most effective approach of transition-based interventions for the senior population. However, research by Hawe, Shiell, \& Riley, (2004) suggests that tailored interventions may be more responsive to the client's context and may be more effective at producing the desired healthcare outcome. Interventions which consider the client's personal experiences, context, skills, and preferences may motivate the individual to participate in the intervention activities. As randomized controlled trials are often

highly standardized, the suitability of tailored interventional research is often questioned. Hawe 
et al. (2004) negate this notion by asserting that although intervention research may comprise of standard activities (i.e. educational material is delivered), they may still be tailored to meet the needs and preferences of the client (i.e., the content varies to suit the client). Therefore, for this review, tailored interventions consisted of individualized intervention components and/or individualized component forms/techniques.

Operational Definition of Approach. Approach was operationalized in terms of whether the intervention was standardized or tailored. Additionally, as complex interventions consisting of multiple components may employ different approached to each respective activity, this review also reported whether a combination of both approaches were used.

\section{Outcome of Interest}

\section{Quality of Care.}

Conceptual Definition of Quality of Care. Quality of care is a multidimensional concept that requires appropriate conceptual and operational definitions to ensure it is assessed accurately. Healthcare definitions of quality are often broad in context and complex in nature (Allen-Duck, Robinson, \& Stewart, 2017). Donabedian (1980) defines quality of care as "the balance of health benefits and harm" (as cited in Gray, Grove, \& Sutherland, 2017, p. 285) and states that quality care incorporates many attributes. The American Medical Association (1994) defined quality as "the degree to which care services influence the probability of optimal patient outcomes" (as cited in Allen-Duck et al., 2017, p. 381). The definition of quality healthcare provided by the Institute of Medicine (IOM) takes a somewhat different stance by acknowledging client preferences (as cited in Gray et al., 2017). The IOM defines quality healthcare as "the degree to which health services for individuals and populations increase the 
likelihood of desired health outcomes and are consistent with current professional knowledge" (as cited in Allen-Duck et al., 2017, p. 381).

Most academic and government definitions of healthcare quality include a description of the defining characteristics and attributes (Allen-Duck et al., 2017). Seven attributes of quality healthcare were identified and defined by Donabedian (1990), and include: (1) efficacy: the ability to improve health; (2) effectiveness: the degree to which attainable health improvements are achieved; (3) efficiency: the greatest health improvement for the lowest cost; (4) optimality: balance of cost and benefits; (5) acceptability: conforming to the patient's preferences regarding many aspects of their care; (6) legitimacy; conforming to the patient's social preferences; and (7) equity: fairness in the distribution of care (as cited in Gray et al., 2017). The Excellent Care for All Act, enacted in 2010, lists nine defining attributes of quality healthcare, including: accessible, appropriate, effective, efficient, equitable, integrated, patient-centred, population health focused, and safe (as cited in Gray et al., 2017). Lastly, the Institute of Medicine (IOM) identifies six quality of care indicators in Canada: safe, effective, efficient, patient-centred, timely, and equitable (as cited in Gray et al., 2017).

Outcomes of healthcare interventions are frequently used as indicators of quality (Dondabedian, 2005). Outcomes of healthcare interventions aimed to uphold quality of care for seniors provide a means of operationalizing quality of care. Healthcare quality for seniors transitioning from acute care to home has been evaluated using the following outcome indicators: patient-centred indicators (using outcomes measures of: patient satisfaction; caregiver distress; patient/caregiver education and involvement in decision making); effectiveness/efficiency indicators (using outcome measures of: hospital length of stay; 30- day readmission rates; total number of days spent in alternative level of care [ALC]; continuity of 
care; complete communication of discharge information); safety indicators (using outcomes measures of: medication reconciliation at discharge); timely indictors (using outcome measures of outpatient follow-up and home care) (Allen et al., 2014; Fox et al., 2013; Health Quality Ontario, 2018; Hyde, Robert, \& Sinclair, 2000; Kansagara et al., 2016; Shepperd et al., 2013).

This review was guided by the six quality of care attributes identified by the Institute of Medicine (IOM): safe, effective, efficient, patient-centred, timely and equitable. Therefore, quality of care was conceptually defined as the degree to which health services for individuals and/or families enhances one or more of the six quality of care indicators outlined by the IOM. The six quality of care attributes may be evaluated based on predetermined healthcare outcomes and standards and may vary between studies.

Operational Definition of Quality of Care. For the purpose of this systematic review, quality of care was operationally defined as the level of change in an indictor (or outcome measure) of quality in the sample being studied. Examples of indictors or outcome measures may include patient-centred, effectiveness, efficiency, equitable, safety, and timely indictors; outcomes measures of each quality indicator may vary between studies. To create a more homogeneous sample, quality of care outcomes were condensed using the following three categories: health service utilization, health status, and self-rated outcomes. Health service utilization outcomes encompassed a variety of measures, including readmission rates, use of social services, and emergency department/primary care visits. Health status outcomes included any health status related measure, such as mortality rates, functional/physical status, nutritional status, heart function, activities of daily living, frailty, drug-related problems, and falls. Selfrated outcomes incorporated any self-rated measure, including the following: self-efficacy change, self-rated symptoms, experiences of safety/security, life satisfaction, self-determination, 
quality of care, self-rated health, caregiver preparedness, patient/caregiver stress, and quality of life.

\section{Relational Statement}

The current body of literature supports the notion that a relationship exists between intervention characteristics and improvements of quality of care for seniors transitioning from acute care to home. Empirical literature suggests that interventions comprised of multiple components are more likely to produce the desired effects (Hsai-mei et al., 2017; Kim \& Thyer, 2015; Kirk, 2014; Le Berre et al., 2017; Mora et al., 2017). Incorporating telephone follow-up and/or home visits in the intervention design may produce improvements in quality of life and rehospitalization outcomes when used in combination with educational components (such as discharge planning), supportive care components (caregiver information) and organizational components (discharge planning) (Hsaio-Mei et al., 2017; Kirk, 2014).

In terms of dose, the reviewed literature suggests a positive linear relationship between the duration of interventions and quality of care (Kim \& Thyer, 2015; Kirk, 2014; Le Berre et al. 2017; More et a., 2017). As the length of treatment intervention increased, improvements in certain quality of care measures (i.e., patient satisfaction), also increased. Additionally, research suggests that interventions delivered using verbal strategies (including face-to-face meetings and telephone follow-up) may increase certain quality of care outcomes (Kirk, 2014; Mora et al., 2017).

The nature of the relationship between intervention characteristics (component, mode, dose, approach) and quality of care remains largely unknown. Intervention characteristics may have a significant impact on the validity of transition-based intervention research (Sidani \& 
Braden, 1998); therefore, an insufficient understanding of the most effective characteristics of interventions may reduce the likelihood of improving quality of care for seniors transitioning from acute care to home.

Figure A displays the relationship among intervention characteristics and quality of care for seniors transitioning from acute care to home. Solid arrows were used to explicate the positive relationship between the type and number of components and the duration of interventions found within the literature. Broken arrows are used for the remaining characteristics, as the relationship between the mode (format, medium), approach (tailored, standardized) and dose (amount, frequency) and quality of care remains unknown. 


\section{Figure A}
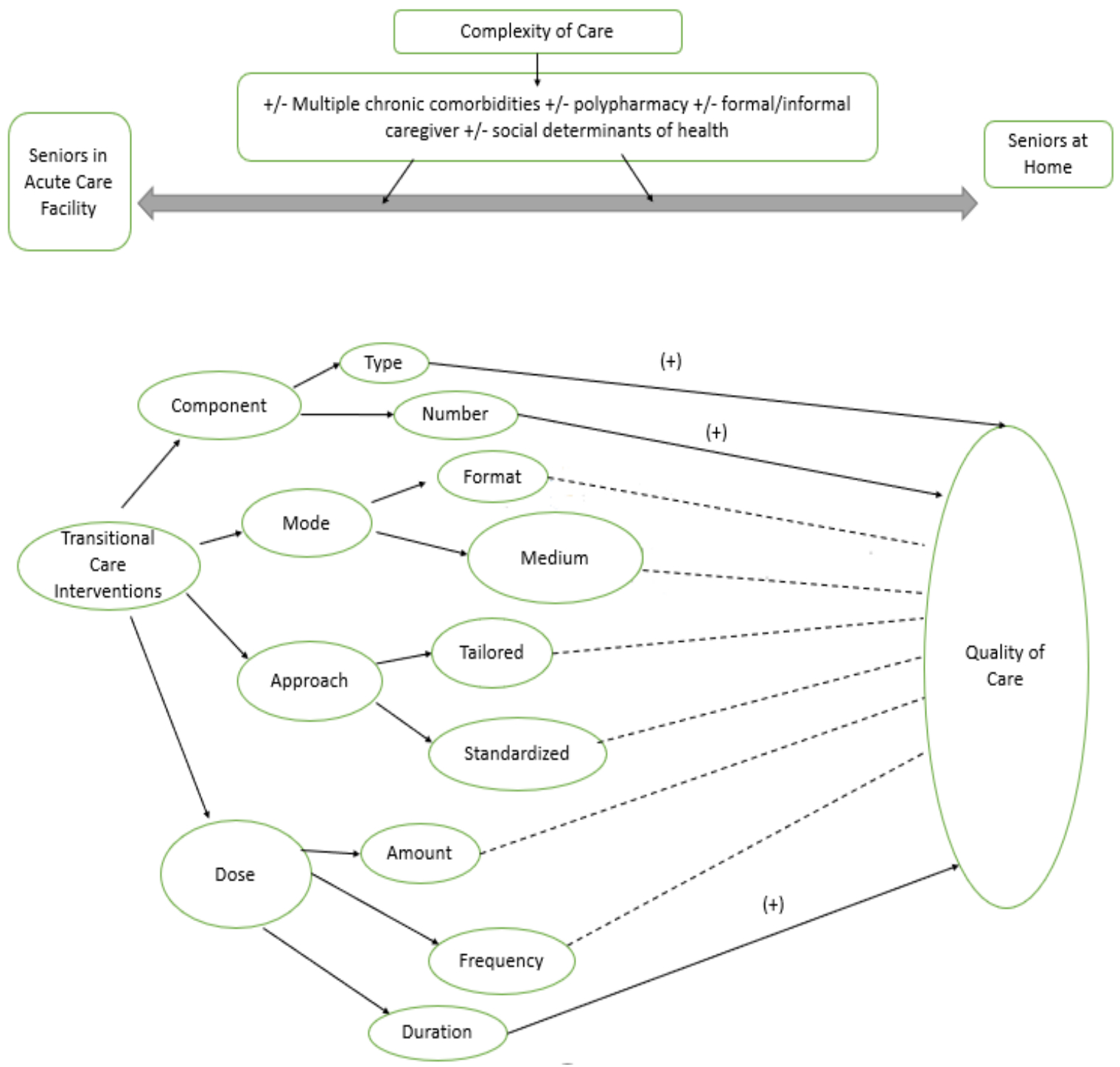

Transition-interventions have been effective in improving both patient-centred and health service utilization measures of quality for seniors (Hsiao-Mei et al., 2017; Kim \& Thyer, 2015; Kirk, 2014; Le Berre et al., 2017; Mora et al., 2017). However, intervention effectiveness varies considerably between studies, and information regarding the optimal characteristics of transitionbased interventions remains largely unknown. This systematic review aimed to better understand the relationship between intervention characteristics (component, mode, dose, and 
approach) and improved quality of care for seniors transitioning from acute care to home. Additionally, data was extracted on the characteristics of the study, participants, and quality of care outcomes.

Descriptive statistics was used to examine the characteristics of the transition-based literature, such as the design, sample size, setting, medical/surgical reason for acute care admission, and quality rating of the research methodology. The most common intervention characteristics aimed to uphold quality of care for seniors transitioning from acute care to home were reported. More specifically, the most common components, mode, dose, and approach were described. Lastly, a description of the most common indicators of quality and tools used represent these quality outcomes, was provided.

\section{Research Questions}

The primary research question was to examine if there are differences in outcomes of quality of care (significance, non-significant, variable) between the subcategories of intervention characteristics. In particular, the following questions were asked:

1) Is there a statistically significant difference in outcomes between interventions based on the number (one, two, three, four) and type (supportive care, educational, medication management, organizational, combination) of component used?

2) Is there a statistically significant difference in outcomes between interventions based on the mode of delivery (medium, format, treatment provider) used?

3) Is there a statistically significant difference in outcomes between interventions based on the dose (amount, frequency, duration, heterogeneity) used? 
4) Is there a statistically significant difference in outcomes between interventions based on the approach (standardized, tailored, combination) used? 


\section{Chapter Four}

\section{Methodology}

This chapter summarizes the study design, selection criteria, and strategies used to search the literature. Additionally, the methods for data extraction and analysis were described.

\section{Study design}

A systematic review of primary transition-based intervention research studies was conducted to examine the characteristics of the interventions. A systematic review is warranted as the most appropriate component, mode, dose, and approach associated with transition-based interventions in enhancing quality of care for seniors remain unknown. Thus, this study intended to systematically search for, appraise, and synthesize the vast transition-based intervention research that currently exists (Grant \& Booth, 2009). This systematic review was conducted to address three objectives. First, to describe the study characteristics of transition-based literature. Second, to report on the most common intervention characteristics. Lastly, the characteristics of transition-based interventions were explored in terms of their effect on multiple quality of care measures designed for seniors. To address these objectives, both descriptive and inferential analysis were used in relation to each intervention characteristics and quality of care outcome. More specifically, chi-square test of independence and Analysis of Variance (ANOVA) was used. To avoid bias, only studies with comparable intervention components, sample characteristics, and outcome measurements were amalgamated and statistically analyzed (Gray et al., 2017; Holly, Salmond, Saimbert, \& Ebooks Corporation, 2012).

Systematic reviews must be conducted using a rigorous search methodology in order to minimize bias and promote the accuracy of results (Gray et al., 2017). To achieve these objectives, this systematic review was guided by the Preferred Reporting Items for Systematic 
Reviews and Meta-analyses (PRISMA) statement (Moher, Liberati, Tetzlaff, \& Altman, 2009).

The PRISMA statement consists of a checklist and flow diagram which assist with the transparent reporting of systematic reviews and meta-analysis. The checklist pertains to the content included in the systematic review; the flow diagram depicts the number of articles, included and excluded, retrieved during the search process, and the reasons for exclusions (Moher, et al., 2009). Please refer to Appendix B for the PRISMA flow and Appendix C for the PRISMA checklist chart which guided this systematic review.

\section{Selection Criteria}

\section{Inclusion Criteria}

To address the study purpose, empirical research studies was deemed relevant for this review based on the following criteria: (1) study reports were written in the English language; (2) the sample consisted of senior patients (65 years or older); (3) the study was a peer-reviewed report published in a scholarly journal within the last decade (2008-2018); (4) the study focused on seniors' transitioning from an acute care inpatient ward (admitted) to home; and (5) The article focuses on transitional care as a whole and/or addresses one or more of the domains of transitional care: discharge planning, complete communication of information, availability, timeliness, clarity, and organization of information, medication safety, educating patient to promote self-management, enlisting help from social and community supports, advance care planning, coordinating care among team members, monitoring and managing symptoms after discharge, outpatient follow-up (Burke et al., 2014). Studies that used experimental or quasiexperimental designs were included in this systematic review.

\section{Rationale for Inclusion Criteria \\ Participants and Setting.}


To address the study purpose, studies were included in this review if they used a sample of seniors ( $\geq 65$ years) within an acute care inpatient ward. Interventions may consider the senior patient, with or without his/her caregiver. Included studies were restricted to seniors transitioning from an in-patient unit to home. Researchers who described interventions that took place solely in the acute care setting or the home environment were considered for this review, as long as the transition from acute care to home was included as part of the intervention protocol. The elimination of other discharge beginning and end points (i.e. transitioning from emergency department to nursing home) was a deliberate choice, to establish a more homogeneous sample and to address the intent of this review.

In order to remain inclusive of the literature and diversity of this population, no limitations were placed on the senior's level of cognition. Additionally, no limitations were set on medical and/or surgical reason for admission, or any social determinant of health (e.g. income, education).

\section{Types of Articles.}

Articles included in this review were required to be written in English to allow for ease of access. Articles were also required to be published in a peer-reviewed scholarly journal. Setting these limitations ensures that articles have undergone the necessary critical appraisal process (Holly et al., 2012). Current literature, including articles published within the last ten years, were included in this review to ensure up-to-date standards of care and transition-based protocols were reported.

Studies had to use experimental or quasi-experimental designs involving two groups in order to be included in this systematic review. Randomized trials have been deemed the most 
robust method of assessing health care interventions, as randomization of group allocation results in a high degree of internal validity (Gray et al., 2017). Quasi-experimental studies were also included in this systematic review, as many health care interventions cannot meet the essential elements of experimental research due to practical and ethical constraints (Rockers, Røttingen, Shemilt, Tugwell, \& Bärnighausen, 2015). Results of meta-analyses demonstrate that the effect sizes of experimental and quasi-experimental studies are comparable (Burns \& Grove, 2005; Rockers, 2015). Therefore, excluding quasi-experimental studies may reduce the overall quality of this systematic review (Rockers, 2015).

Lastly, articles were chosen for this review if they addressed one or more domain of transition-based care (e.g. discharge planning, interprofessional communication, medication safety, patient education). The operational definition of transition-based interventions provided in chapter three guided the review process. Interventions that addressed either a single transition-domain or multiple domains of transitional care were eligible for this review.

\section{Exclusion Criteria}

Research studies were excluded from this review based on the following exclusion criteria: (1) the intervention was designed for a health care provider and (2) secondary analyses.

\section{Critical Appraisal Tool}

This systematic review incorporated an appraisal process to assess the overall methodological rigour of each included study. Each article included in this systematic review was subjected to assessment by one critical appraiser. Additionally, three articles were chosen at random and evaluated by a separate appraiser to ensure consistency and accuracy. The Quality Assessment Tool for Quantitative Studies, developed by the Effective Public Health Practice 
Project (EPHPP), is the instrument that guided the evaluation of each included article (See Appendix D). The EPHPP tool provided an overall methodological rating of weak, moderate, or strong based on eight sections, which evaluated the following: selection bias, study design, confounders, blinding, data collection methods, withdrawals and dropouts, intervention integrity, and analysis (EPHPP, 2010). This tool has been assessed for content validity and reliability using an iterative process.

\section{Literature Search Strategy}

A search of the literature was conducted to identify pertinent empirical studies that addressed interventions for seniors transitioning from acute care to home. The systematic search for relevant literature was performed using the five electronic databases, including Cumulative Index to Nursing and Allied Health Literature (CINAHL), Medline, ProQuest, PsychInfo, and HealthStar. The following search terms guided the review: aged, aged 80 and over, intervention, transitional care, acute care, hospitalized, nursing care, gerontologic care, and supportive care. The Boolean operators AND and OR were used. A supplemental search of the reference lists of all included literature was also conducted. The search was conducted with the assistance of an expert university librarian.

A general search strategy was carried out and applied to each database. Keywords relating to older adults, including "aged" and "aged 80 and over" were entered and combined with the operator OR. In a separate search, key terms relevant to transition ("transitions of care", "care transitions", and "continuity of patient care") were also conducted, using the operator OR to separate each keyword. Another search was done with key words related to interventions (“intervention", "nursing intervention", "program", and "treatment"). Lastly, key terms related to the acute care setting ("acute care", "hospitalized") were combined with the operator OR. The 
results of these four separate searches was then combined using the operator AND to yield a total number of potential articles to review for relevance to this systematic review. This search strategy was repeated for each research index. A supplemental search of the reference lists of retrieved articles was conducted manually for relevant studies. Articles were limited to papers written in English and published within the last decade.

\section{Data Extraction Strategy}

Data were extracted on study characteristics, characteristics of transition-interventions, and outcomes of quality of care. The extracted data were compiled into a data collection table to describe participants as well as characteristics of the study, such as the quality assessment rating of included articles. The definitions provided in chapter three guided the development of a coding scheme to facilitate data extraction from each article. The following section provides details pertaining to the coding scheme for this systematic review.

\section{Study Characteristics}

The following information was collected to describe each study: author's name(s), year of publication, country in which the study was done, study design (non-experimental, quasiexperimental, experimental, pilot study), and total sample size. Results of the EPHPP assessment tool (weak, moderate, and strong) and global rating score results (for weak/moderate scores) were included to describe the methodological rigour of the study design. Characteristics of the sample population, including admitting diagnosis, setting (admitting ward), and age were also extracted. These data were used for descriptive purposes.

\section{Characteristics of Interventions}


Data were extracted related to intervention characteristics, including component, mode, dose and approach and the outcome of interest, that is, quality of care:

\section{Component.}

The number and type of components were identified from the description of the intervention. Intervention components were categorized as either educational, supportive care, medication management, organizational, or a combination of these components. The type of components was coded as: 1) educational; 2) supportive care; 3) medication management; 4) organizational; or 5) combination of two or more components. The number of components in a given intervention were counted and reported. Please refer to Appendix I for a detailed description of each component type.

\section{Mode.}

The mode of interventions was considered in term of the medium and format through which the intervention was given. The medium was coded as 1) face-to-face contact with a health care professional; 2) phone contact with health care professional; 3) distribution of written resources (including pamphlets, booklets, brochures, or handouts); and 4) a combination of these strategies.

The format for delivery of transition-interventions was coded as: 1) one- to- one delivery of intervention provided to the senior alone; 2) group delivery involving interventions provided to the senior and caregiver, family, or support person; and 3) mix of both strategies. Format was further categorized by the healthcare provider delivering the intervention. This was coded as 1) registered nurse; 2) multidisciplinary team; or 3) single disciplinary member of the healthcare team (e.g. social worker, physiotherapist, occupational therapist). 


\section{Dose.}

The dose was indicated by the amount, frequency, and duration of the intervention. Amount referred to the length of each session, recorded in minutes; amount values were averaged if multiple measures of this dose value were described. Frequency was reported by the number of times the sessions were given per month. Frequency was coded as variable if the dose schedule changed throughout the study. Duration was indicated by the total time period in which the sessions were provided, in weeks. Duration intervals were based on the observed data from the included interventions and was coded as 1) one to three months; 2) four to six months; 3) seven to twelve months.

To account for potential heterogeneity of the certain dose measures (amount and frequency) additional variables were created, including: 1) fixed; 2) variable and 3) tailored; 4) untailored. Whereas a fixed dose schedule is constant during the intervention, or variable, in which the dose schedule may change throughout the study. Additionally, dose schedules were coded as tailored, that is, given in accordance to the senior's needs, or untailored, in which every senior involved in the study receives the same treatment dose. Full definitions can be found in Chapter Three.

\section{Approach.}

The approach to transition-based interventions was obtained from the description of the intervention and/or the procedure for delivering it. Interventions were categorized as standardized if they involved the following activities: video tapes, audiotapes, pre- designed discharge resources, standardized telephone follow-up questions. Interventions designed specifically for individual patients' needs, such as tailored discharge plans, resources, and 
procedures, were considered tailored. Approach was coded as 1) standardized; 2) tailored; or 3) combination of both standardized and tailored strategies.

\section{Outcomes of Transitional Care Interventions.}

The specific outcomes of interest, that is, indicators of quality of care, may be evaluated based on a variety of healthcare outcomes. Therefore, outcomes were categorized using three distinct groups, including 1) health service utilization; 2) health status outcomes; and 3) selfrated outcomes. Health service utilization outcomes encompassed a variety of measures, including readmission rates, and emergency department visits, and primary care visits. Health status outcomes included any health status related measure, such as mortality rates, functional/physical status, nutritional status, activities of daily living, frailty, drug-related problems, and falls. Self-rated outcomes incorporated any self-rated measure, including the following: quality of life, self-efficacy change, self-rated symptoms, experiences of safety/security, life satisfaction, quality of care, self-rated health, caregiver preparedness, patient/caregiver stress, and quality of life.

Using these three categories, data were extracted on the presence of statistically significant differences between groups or over time; this information was recorded as $1=$ nonstatistically significant; $2=$ statistically significant; or $3=$ variable for each primary outcome. Interventions which evaluated two indicators (e.g. readmission rates and primary care visits) of the same outcome category (e.g. health service utilization) and yielded significant results in one indicator and non-significant in the other, were categorized as variable.

\section{Data Analysis}

Descriptive statistics were used to report the following information: 
1) Describe the characteristics of studies and participants included in this review: nominal and ordinal data were expressed as percentages; interval and ratio data were expressed using measures of central tendency.

2) Describe the types of quality of care indicators used amongst studies: indicators were presented as percentages, frequency distributions, measures of central tendency.

3) To report on the most commonly reported intervention component, mode, dose, and approach among the included studies: information pertaining to intervention characteristics was presented as percentages, frequency distributions, measures of central tendency.

To address the second objective, that is, to identify which intervention characteristics are associated with a statistically significant changes in measures of quality of care, inferential statistics was used. A chi-square test of independence was conducted for all nominal level data to evaluate whether proportions in levels of one variable are significantly independent from proportions of the second variable. Health service utilization, health status, and self-rated outcomes were analyzed with component (type), mode (medium and format), dose (heterogeneity), and approach (standardized and tailored). Nominal level dependent variables and ratio level independent variables were analyzed using inferential statistics; an ANOVA test assessed for differences in health service, health status, and self-rated outcomes, among each of the subcategories of intervention characteristics: the number of components within an intervention, and dose (length of intervention in minutes), frequency (number of times the intervention occurred per month), and the duration of the intervention (reported in months). 


\section{Inter-rater Reliability}

Inter-rater reliability is warranted to eliminate ambiguity and increase the rigor of the systematic review (Gray et al., 2017; Holly et al., 2012). The primary researcher provided the second-rater with written instructions pertaining to the methods of data extraction for the following information: study characteristics, participant characteristics, intervention characteristics, and outcomes of interest. The second-rater then independently collected and extracted data on ten percent of the randomly selected research articles for comparison. A value of 0.80 or greater was deemed appropriate for this review (Gray et al., 2017). Discrepancies were discussed, and if necessary, resolved with a third reviewer (Holly et al., 2012). Overall, the inter-rater reliability was $100 \%$ amongst the two reviewers. 


\section{Chapter Five}

\section{Results}

Findings of this systematic review are presented in this chapter in the order of the three research questions. The design and sample characteristics of included studies were first described. Then, reports of the most common intervention characteristics were provided. Quality of care indicators were then described in terms of the most commonly reported outcome indicators, measurement tools used, and reports of reliability/validity. Finally, the primary research question, to examine the differences between intervention characteristics and outcomes of interest, was addressed. Descriptive and inferential statistics (e.g. chi-square, analysis of variance) was used to examine the relationship between intervention characteristics and quality of care.

The search strategy resulted in 2,435 eligible studies (see Appendix E for a detailed description of the search results). Screening of the title/abstracts resulted in 2,158 studies being eliminated. This resulted in 277 full-text articles which were then reviewed for relevancy based on the inclusion and exclusion criteria described in Chapter Four. Of these 277 studies, an additional 243 were eliminated for the following reasons: non-experimental $(n=22)$, participants under the age of 65 years $(n=80)$, did not report on transitional care interventions $(n=51)$, not acute care to home $(n=67)$, and a combination of more than one of the aforementioned criteria $(n=23)$. In addition to this process, a manual search of the reference lists of review studies resulted in four suitable articles. Therefore, a total of 38 articles met the described inclusion/exclusion criteria and were included in this review. See Appendix F for a summary of the included articles.

\section{Description of Studies}


Seventeen (44.7\%) of studies were of strong methodological quality, twelve (31.6\%) were moderate, and the remaining nine (23.7\%) weak. Moderate and low-quality ratings were commonly due to failure to blind outcome assessors/study participants ( $\mathrm{n}=17,44.7 \%)$ and selection bias $(\mathrm{n}=10,26.3 \%)$. Of the 38 included studies, $31(81.6 \%)$ used a randomized controlled trial design to evaluate the effectiveness of transition-based interventions, while seven (18.4\%) of the studies used a pilot study design. Approximately 17 (44.7\%) studies were measured on two occasions (post-intervention and follow-up). The year of publication ranged from 2008 to 2018. Studies were conducted in Europe ( $\mathrm{n}=17,44.7 \%)$, Australia $(\mathrm{n}=8,21.1 \%)$, the United States of America ( $\mathrm{n}=6,15.8 \%)$, and Asia $(\mathrm{n}=7,18.4 \%)$.

\section{Description of Participants}

The 38 studies that met the inclusion criteria for this systematic review included a total of 10, 924 participants. Participants in the control group were on average 79.8 years of age, with individuals in the experimental group averaging an age of 79.6 years. Approximately 11 (29\%) studies took place on a medical unit, five $(13.1 \%)$ on a geriatric unit, and three $(7.8 \%)$ on medical/surgical units. Most studies $(n=26,68.4 \%)$ did not limit their sample based on a specific medical/surgical condition. Of the studies that did limit their sample to participants who were admitted for a specific health-issue, six $(15.7 \%)$ were cardiac, four $(10.5 \%)$ were nutritional health risk, one (2.6\%) was stroke, and one (2.6\%) was hip fracture patients. Please see Appendix G for a summary of the study/participant characteristics.

\section{Patterns Across Intervention Characteristics}

The most common intervention characteristics aimed to uphold quality of care for seniors transitioning from acute care to home is detailed below. Although 38 studies were included in this review, several studies $(n=6,15.7 \%)$ described the use of more than one experimental group 
in their study design; four (10.5\%) studies used two distinct experimental groups while two studies (5\%) used three. In order to analyze the interventions based on their specific characteristic, each experimental group was considered separately. Therefore, this section will detail the intervention characteristics of each of the 46 transitional care experimental groups. Please refer to Appendix $\mathrm{H}$ for a detailed summary of the intervention characteristics across the 46 interventions.

\section{Component}

Most interventions $(\mathrm{n}=35,76.1 \%)$ used a combination of two or more components. Interventions that used only one component $(23.9 \%, n=11)$ were either educational $(n=3,6.5 \%)$, supportive care $(n=7,15.2 \%)$, or medication management $(n=1,2.2 \%)$. The number of components the intervention comprised of ranged from one to four. Interventions most often used two components $(n=29,63 \%)$, followed by one $(n=11,23.9 \%)$, three $(n=4,8.7 \%)$, and four $(n=2,4.3 \%)$. Of the $29(63 \%)$ interventions that used two components, the most common combinations were organizational and supportive care $(n=14,48.3 \%)$, educational and supportive care $(n=12,41.3 \%)$, and medication management and supportive care $(n=3,10.3 \%)$.

All multi-component interventions incorporated a supportive care component $(n=35$, $100 \%)$. This was followed by organizational components $(n=17,48.6 \%)$, educational components $(n=17,48.6 \%)$, and medication management strategies, which were described in $25.7 \%(n=9)$ of multicomponent interventions. Please see appendix I for descriptions of each component types (educational, supportive care, organizational, medication management). Additionally, Appendix O contains information regarding the combination of components found within the literature and the effect on indicators of quality of care. 


\section{Mode}

The majority $(n=23,50 \%)$ of interventions used a combination of strategies to deliver transitional care. Of the 23 interventions which used a combination of strategies, 22 (95.6\%) incorporated a face-to-face approach. Phone contact was comparable, being reported in approximately $(\mathrm{n}=21,91.3 \%)$ of interventions which used a combination of delivery strategies. Additionally, of the interventions that used a combination of strategies $(n=23)$, most $(n=16$, $69.5 \%$ ) used two strategies. Face-to-face and telephone contact was the most common combination employed $(n=12,52 \%)$. Of the remaining 23 interventions which used only one mode of delivery, most reported face-to-face strategies $(n=16,69.5 \%)$ or phone contact $(n=6$, $26 \%)$

Interventions were commonly delivered solely to the patient ( $\mathrm{n}=28,60.9 \%)$; however, group sessions were also commonly reported $(n=17,36.9 \%)$. Interventions reported similar numbers regarding treatment providers. Treatments were frequently delivered by nurses $(\mathrm{n}=11$, $23.9 \%)$ or the multidisciplinary $(n=17,36.9 \%)$ team. The most common treatment providers amongst interventions were other allied health professions $(n=18,39.1 \%)$ which included physiotherapists, occupational therapists, dietitians, practitioners, and social workers. Please see Appendix $\mathrm{P}$ for a detailed description of the combination of mode types found within the literature and their effect on indicators of quality of care.

\section{Dose}

Dose amount (in minutes) was the least consistently reported intervention characteristic overall. Of the 13 interventions that reported on this dose measure (out of 46), the average amount of time the treatment was delivered was approximately $60(S D=38)$ minutes. Dose 
amounts ranged from 15 to 120 minutes. The amount of times the intervention was delivered per month (dose frequency) was reported in $93.5 \%(n=43)$ interventions. The majority $(n=19$, $41.3 \%$ ) of interventions reported dose schedules that varied in frequency. Of the interventions that used a more standardized approach, the most common dose frequencies were one $(\mathrm{n}=7$, $15.2 \%)$ and four $(n=7,15.2 \%)$ times per month. The duration of the intervention ranged from one $(n=19,41.3 \%)$ to twelve months $(n=6,13 \%)$, with the most common being the former.

\section{Approach}

The approach of an intervention refers to the structure in which the intervention is given. Of the 46 included interventions, most interventions used a standardized approach $(n=21$, 45.7\%). A combination of standardized and tailored strategies was reported in 17 (37\%) interventions.

\section{Indicators of Quality of Care}

Outcomes were grouped into three distinct categorizes, health service utilization, health status, and self-rated outcomes. Health service utilization outcomes were used in $50 \%(\mathrm{n}=23)$ of transition-based interventions. Outcome indicators included readmission rates $(\mathrm{n}=22,44.7 \%)$, emergency department visits $(n=3,7.8 \%)$, and primary care visits $(n=3,7.8 \%)$. Health status outcomes were also reported in $(n=23,50 \%)$ of included interventions and comprised any health status related measure, such as functional/physical status $(n=14,34.2 \%)$, activities of daily living $(n=6,13 \%)$, falls $(n=3,10.5 \%)$, mortality rates $(n=3,7.9 \%)$, drug-related problems $(n=2$, $5 \%)$, nutritional status $(\mathrm{n}=1,2 \%)$, and frailty $(\mathrm{n}=1,2 \%)$. Self-rated measures were the least likely outcomes used amongst interventions $(n=13,28.3 \%)$. Self-rated outcomes incorporated any self-rated measure, including the following: quality of life $(n=6,15.7 \%)$, self-efficacy change $(n=2,5 \%)$, self-rated symptoms $(n=1,2 \%)$, experiences of safety/security $(n=2,5 \%)$, 
life satisfaction $(n=1,2 \%)$, quality of care $(n=1,2 \%)$, self-rated health $(n=1,2 \%)$, caregiver preparedness $(n=1,2 \%)$, and patient/caregiver stress $(n=1,2 \%)$. Appendix $J$ provides a detailed summary of the outcome indicators included in each of the three categories; additionally, appendices L, M, and N provide an overview of studies yielding statistically significant results, non-significant results, and variable results based on each intervention characteristic.

Of the 39 interventions that used an instrument to measure outcome(s), most $(n=38$, $97.4 \%$,) reported on the reliability and validity of the measurement tool. One intervention $(2.5 \%)$ used an outside source to provide details regarding the instrument's validity/reliability. Due to the large range of outcomes amongst interventions, measurement tools varied considerably between interventions. The Barthel-100 Index Score, which was used to measure physical status (i.e. Activities of daily living) and the Minnesota Living with Heart Failure Questionnaire, used to measure self-rated measures (i.e. health related quality of life) were among the most commonly used measurement tools (used in 6 and 3 interventions, respectfully).

\section{Primary Research Question}

This section will present findings related to the primary research question outlined in chapter three: to determine if the component (number and type), mode (medium and format), dose (amount frequency, duration, and heterogeneity), and approach, of interventions is associated with improvements in a variety of quality of care indicators.

Outcome categories (health-service utilization, health status, and self-rated) were reported in terms of their effect, being either significant, non-significant, or variable.

Interventions which evaluated two indicators (e.g. readmission rates and primary care visits) of the same outcome category (e.g. health service utilization) and yielded significant results in one 
indicator and non-significant in the other, were categorized as variable. Please refer to Appendix $\mathrm{K}$ for a detailed description of the combinations of outcome categories.

For each of the intervention characteristics, findings were presented in the following pattern. Results obtained from descriptive analysis were provided for all interventions $(n=46)$, in relation to each intervention characteristics and each outcome category. Then, results obtained from inferential statistics were reported. Specifically, chi-square test of independence was used to examine the relationship between sub-categories within each intervention characteristic and study outcomes. Due to the small number of studies with variable outcomes within each intervention characteristic, all chi-square analyses were limited to interventions with significant or non-significant findings ( $\mathrm{n}=43$ ). Please see Appendix $\mathrm{Q}$ for all chi-square results. Lastly, an analysis of variance (ANOVA) test was used for the ratio level intervention characteristics (i.e. the number of components within an intervention, intervention dose interval). For instance, the ANOVA examined any differences among the intervention dose intervals and study outcomes. Please see Appendix R for the results of the ANOVA tests.

\section{Component}

Component Type. The type of component was described in $100 \%$ of the interventions included in this review. Please see Appendix $\mathrm{O}$ for information regarding the combination of components found within the literature and the effect on indicators of quality of care.

No interventions evaluating health service utilization were comprised of a single educational/medication management component. Two interventions comprised of a single supportive care component and evaluated health service utilization, with one indicating nonsignificant findings $(\mathrm{n}=1,2.2 \%)$ and one reporting variable results $(\mathrm{n}=1,2.2 \%)$. A combination 
of two or more components yielded significant findings in $12(26.1 \%)$ interventions and nonsignificant findings in nine $(19.6 \%)$ of interventions which evaluated health service utilization.

In relation to health status outcomes, interventions using an educational component yielded significant $(n=1,2.2 \%)$ and non-significant $(n=2,4.3 \%)$ findings. Additionally, two (4.3\%) interventions that incorporated a supportive care component indicated non-significant findings, variable results in three (6.5\%) interventions, and significance results in one (2.2\%) intervention. One intervention $(2.2 \%)$ was comprised of a single medication management component and used health status outcomes to evaluate quality of care; this study indicated nonsignificant results. Interventions yielded significant $(n=3,6.5 \%)$ or variable $(n=3,6.5 \%)$ results when using a combination approach; non-significant findings were reported in seven (15.2\%) interventions which used a combination of components in their intervention design.

When considering self-rated outcomes, no interventions used a single educational component. Those employing supportive care components yielded significance in one (2.2\%) intervention and non-significance in two (4.3\%). A single medication management component was used in one intervention (2.2\%), which indicated non-significant findings. Using a combination approach, significance was yielded in seven (15.2\%) interventions and nonsignificant findings were reported in two (4.3\%).

A chi-square test was used to assess for a relationship between the type of component(s) (i.e., supportive care, medication management, organizational, educational, or a combination) and findings (statistical significance or non-significance) for each of the three outcome categories (i.e. health service utilization, health status, and self-rated outcomes). Non-significant findings were noted in relation to health status utilization outcomes $\left(\chi^{2}{ }_{(1)},=1.26, p=.262\right)$, 
health status $\left(\chi_{(3)}=0.463, p=.927\right)$, and self-rated outcomes $\left(\chi^{2}{ }_{(2)}=0.164, p=.164\right)$. Please see Appendix Q for all chi-square results).

Component Number. Overall, studies with significant findings employed interventions with multiple components. Of the 46 interventions included in this review, only 16 yielded significant results in each outcome under investigation. Of these, 15 (93.75\%) were multicomponent interventions. More specifically, within these 16 interventions, the majority $(n=13)$ of treatments were comprised of two components, while one treatment used four components, and one used three components within their intervention design. Only one intervention (6.2\%) used a single component and reported statistical significance in each primary outcome.

However, results from an analysis of variance did not yield significant findings. An analysis of variance was completed to examine the differences between the number of components within an intervention and study findings. Non-significant results were indicated for health service utilization $[F(3,22)=0.334, p=0.801]$, health status $[F(2,22)=0.787, p=0.469]$, and self-rated outcomes $[F(2,12)=1.838, \mathrm{p}>0.209]$. Please see Appendix $\mathrm{R}$ for all results from the ANOVA tests.

\section{Mode.}

Mode: Medium. The way in which the intervention was implemented was reported in $100 \%$ of the interventions included in this review. Please see Appendix P for information regarding the combination of mode types found within the literature and the effect on indicators of quality of care.

In terms of health service utilization outcomes, significant findings were identified in two (4.3\%) of interventions which used a face-to-face strategy and non-significant findings were reported in five $(10.9 \%)$ of the literature. Alternatively, one $(2.2 \%)$ intervention employing 
phone contact strategies indicated significant results, while two (4.3\%) indicated non-significant findings, and one (2.2\%) of the literature yielded variable results. Written materials yielded significance in zero $(0 \%)$ interventions and non-significance in one $(2.2 \%)$ of intervention. Using a combination approach yielded significance in $19.6 \%(n=9)$, while $4.3 \%(n=2)$ of interventions indicated non-significant findings.

In relation to health status outcomes, using a face-to-face mode of delivery yielded significant findings in four $(8.7 \%)$ interventions, non-significant findings were reported in five $(10.9 \%)$, and variable results were indicated in two (4.3\%) interventions. Additionally, two (4.3\%) interventions that incorporated phone contact reported non-significant findings and zero $(0 \%)$ of interventions indicated significance. No interventions using written materials in their intervention design reported on health status outcomes. Additionally, one (2.2\%) intervention yielded significant findings and four $(8.7 \%)$ interventions yielded variable results when using a combination approach; non-significant findings were reported in five (10.9\%) interventions which used a combination of delivery methods in their design.

In terms of self-rated outcomes, using a face-to-face mode of delivery yielded significant findings in two $(4.4 \%)$ interventions and non-significant findings were reported in one (2.2\%) intervention. Additionally, one (2.2\%) intervention that incorporated phone contact indicated non-significant findings and one $(2.2 \%)$ reported significant findings. No interventions using written materials in their intervention design reported on self-rated outcomes. Approximately five $(10.9 \%)$ of interventions yielded significant findings when using a combination approach; non-significant findings were reported in three (6.5\%) interventions which used a combination of strategies in their intervention design. 
To assess for a relationship between the way the intervention was delivered and statistically significant findings in relation to the three outcome categories, a chi-square test was conducted. Non-significant findings were noted in relation to health status utilization outcomes $\left(\chi^{2}{ }_{(3)}=6.949, p=0.074\right)$, health status $\left(\chi_{(2)}=2.282, p=0.319\right)$, and self-rated outcomes $\left(\chi^{2}{ }_{(2)}\right.$ $=0.149, p=0.928)$. Please see Appendix $\mathrm{Q}$ for all chi-square results.

Mode: Format. In terms of health service utilization outcomes, significant findings were identified in eight $(17.4 \%)$ interventions delivered on a one-to-one basis and non-significant findings were reported in seven (15.2\%) interventions. Significant findings were identified in four $(8.7 \%)$ interventions which described group-based treatments, while variable findings were reported in one $(2.2 \%)$. Alternatively, non-significant results were reported in three $(6.5 \%)$ group-interventions. A combination of one-to-one and group strategies were not used in interventions reporting on health service utilization outcomes.

In relation to health status outcomes, employing a one-to-one strategy yielded statistical significance in four $(8.7 \%)$ interventions and non-significance in nine $(19.6 \%)$. Variable resulted were reported in three $(6.5 \%)$ treatments which employed a one-to-one approach. Additionally, three $(6.5 \%)$ interventions that incorporated group-delivered strategies were non-significant, one (2.2\%) intervention was significant, and two (4.3\%) yielded variable results. A combination approach to intervention delivery was employed in one $(2.2 \%)$ intervention, yielding variable results.

When considering self-rated outcomes, significant results were indicated in two $(4.3 \%)$ interventions using a one-to-one approach, while non-significant findings were reported in three $(6.5 \%)$. Significant findings were identified in five (10.9\%) interventions using a group-mode of 
delivery and non-significance in two (4.3\%). Using a combination approach was used in one intervention $(2.2 \%, \mathrm{n}=1)$ evaluating self-rated outcomes, indicating significant findings.

A chi-square test was used to assess for a relationship between who the intervention was delivered to and statistically significant findings in relation to the three outcome categories. Nonsignificant findings $(p>0.05)$ were noted in relation to health status utilization outcomes $\left(\chi 2_{(1)}=\right.$ $0.028, p=0.867)$, health status $\left(\chi_{(1)}=0.049, p=0.825\right)$, and self-rated outcomes $\left(\chi_{(2)}=01.894\right.$, $p=0.388)$. Please see Appendix $\mathrm{Q}$ for all chi-square results.

Mode: Treatment Provider. In terms of outcome one (health-service utilization), significant findings were identified in seven (15.2\%) interventions delivered by a nurse and nonsignificant findings were found in two (4.3\%). Interventions delivered by the multidisciplinary team yielded significant findings in four $(8.7 \%)$ interventions and non-significant results were reported in four $(8.7 \%)$. Treatments delivered by other members of the healthcare team yielded significant findings in one $(2.2 \%)$ intervention, variable results in one $(2.2 \%)$, and nonsignificant findings in four $(8.7 \%)$ interventions.

In relation to health status outcomes, statistical significance was indicated in zero $(0 \%)$ interventions led by nurses, and non-significance was identified in one (2.2\%) intervention. Variable results were found in two (4.3\%) interventions led by a nurse. Additionally, one (2.2\%) intervention using a multidisciplinary approach was non-significant, two (4.3\%) were significant, and three $(6.5 \%)$ yielded variable results. Significant findings were identified in three $(6.5 \%)$ interventions led by other healthcare providers, non-significant findings were found in 10 $(21.7 \%)$, and variable in one $(2.2 \%)$. 
When considering self-rated outcomes, those led by nurses yielded significant results in two $(4.3 \%)$ interventions, while non-significant findings were reported zero $(0 \%)$. Interventions employing a multidisciplinary approach of delivery yielded significance in six (13.3\%) interventions and non-significance in one (2.2\%). Interventions led by other members of the allied healthcare team reported significant findings in zero $(0 \%)$ and non-significant findings in four $(8.7 \%)$ interventions.

A chi-square test was used to assess for a relationship between the who delivered the interventions and the outcome findings. Statistically significant findings $\left(\chi_{(2)}=9.379, p<0.05\right)$ were yielded in relation to self-rated outcomes and treatment providers. More specifically, a standardized residual test was conducted and showed a statistically significant relationship between the observed and expected values for the following combination: treatment providers (single member of the disciplinary team) and non-significant results. Non-significant findings were noted in relation to health status utilization outcomes $\left(\chi^{2}{ }_{(2)}=4.433, p=0.109\right)$ and health status $\left(\chi^{2}(2)=2.674, p=0.263\right)$. Please see Appendix $\mathrm{Q}$ for all chi-square results.

Dose. Interventions which produced statistically significant results in each primary outcome (16 out of 46) were not likely to report on the amount of time in minutes the treatment lasted. Only two of the 16 effective interventions provided this dose measure, both of which described treatments sessions which lasted 45 minutes. Additionally, the amount of times an intervention was delivered ranged from one to five times per month., 19 interventions (41\%), used a variable approach to intervention implementation. Effective treatments were more likely $(\mathrm{n}=8$ out of $16,50 \%)$ to include pre-and-post discharge procedures. Lastly, the majority $(\mathrm{n}=8$ out of $16,50 \%$ ) of significant interventions lasted 4-weeks in duration; however, effective treatments ranged from one week to one year, lasting an average of 116 days. 
Alternatively, within the 13 interventions (out of 46) which reported non-significant findings in all primary outcomes, 10 described the duration of the intervention. Non-significant interventions ranged from 20 days to one year, with the most common being two months $(\mathrm{n}=3)$ or twelve-month $(\mathrm{n}=3)$ treatments. On average, ineffective treatments lasted 85 days. The majority ( $\mathrm{n}=11$ out of $13,84.6 \%)$ were limited to either pre-or-post discharge transitional care procedures.

Dose: Amount. With regards to the amount of time (in minutes) the intervention was delivered, an analysis of variance was completed to examine the differences between the following groups: not reported, 15, 35, 45, 60, and 120 minutes. Groups were determined by the dose intervals described across interventions.

When comparing the differences in health service utilization outcomes with the dose amount (in minutes), non-significant findings were reported $[F(5,22)=1.087, p=0.403]$. The amount of time was non-significant $[F(5,22)=0.350, p=0.875]$ when compared with the differences in health status outcomes. The differences in self-rated outcomes also yielded nonsignificant results $[F(1,12)=0.114, p=0.742]$. Please see Appendix $\mathrm{R}$ for all ANOVA results.

Dose: Frequency. With regards to the number of times an intervention was delivered per month, an analysis of variance was completed to examine the differences among the following groups: not reported, once per month, twice per month, three times per month, four times per month, five times per month, or variable. ANOVA showed non-significant findings in the number of health service utilization $[F(6,22)=2.081, p=0.113]$, health status $[F(6,22)=1.421$, $p=0.267]$, and self-rated outcomes $[F(4,12)=1.077, p=0.428]$ when compared among the various intervals of intervention frequencies. Please see Appendix R for all ANOVA results. 
Dose: Duration. With regards to the length of time (in months) the intervention occurred, an analysis of variance was completed to examine the differences between the following groups: one- three months ( $n=32$ out of $43,74.4 \%$ ), four-six months ( $n=5$ out of $43,11.6 \%$ ), and seventwelve months ( $\mathrm{n}=6$ out of $43,13.9 \%$ ). The three distinct duration groups were categorized in this way as most interventions were reported in months or were able to be converted into months. Three interventions ( $\mathrm{n}=3$ out of 46 ) did not report on dose duration and were not included in the analysis.

The results of the ANOVA yielded non-significant findings when examined with health service $[F(2,21)=0.309, p=0.728]$ and self-rated $[F(2,11)=0.370, p=701]$ outcomes when compared with the various duration periods. A statistical significant difference in health status outcomes was noted among the various intervals of intervention duration $[F(2,20)=4.344$, $\mathrm{p}<0.05]$. Please see Appendix R for all ANOVA results. However, a post hoc test was not possible as the sample sizes were too small. More specifically, one group (interventions between four-and-six months duration) were only reported in one case. As such, the pattern of findings among intervals of intervention dose duration was described below to further explore the statistically significant findings on health status outcomes.

Of the 21 interventions which evaluated health status outcomes and reported dose duration, five (23.8\%) yielded statistically significant results. Of which, all five were between one and three months in duration. More specifically, interventions lasted 4 weeks in duration $(\mathrm{n}=3)$, two months $(\mathrm{n}=1)$ and three months $(\mathrm{n}=1)$ in duration. Additionally, three studies (out of 21, 14.2\%) had treatment durations between four and 12 months, all of which yielded variable results. Details on studies with variable findings are described in Appendix N. 
Dose: Heterogeneity. Dose heterogeneity was reported in 41 (89\%) of the included interventions $(\mathrm{n}=46)$. In terms of health service utilization outcomes, significant findings were identified in eight (17.4\%) interventions which used a fixed dose schedule, variable results were reported in one (2.2\%), and non-significant results in nine (19.6\%). A variable dose schedule yielded significance in four interventions $(8.7 \%)$ and non-significant results in one (2.2\%). Additionally, tailored dose schedules resulted in significance in four (8.7\%) interventions, nonsignificance in one (2.2\%), and variable results in one (2.2\%). Untailored dose schedules yielded significance in eight (17.4\%) interventions and non-significance in nine $(19.6 \%)$.

In terms of health status outcomes, a fixed dose schedule yielded significant results in four (8.7\%) interventions, variable results in three (6.5\%), and non-significant findings in seven (15.2\%). A variable dose schedule yielded non-significant results in zero (0\%) interventions, significance in one (2.2\%), and variable three (6.5\%). Additionally, tailored dose schedules resulted in significance in zero (0\%) interventions and non-significance in five (10.9\%). Untailored dose schedules yielded significant findings in five (10.9\%), non-significance in four $(8.7 \%)$, and variable results in three $(6.5 \%)$.

In relation to self-rated outcomes, a fixed dose schedule yielded significant findings six (13.3\%) interventions and non-significant findings in three (6.5\%). Employing a variable dose schedule yielded significance in two (4.3\%) interventions and non-significance in one (2.2\%). Tailored dose schedules resulted in significant findings in four (8.7\%) interventions and nonsignificance in three (6.5\%). Untailored dose schedules yielded significance in four (8.7\%) and non-significance in one $(2.2 \%)$ intervention.

A chi-square test was used to assess for a relationship between the dose heterogeneity (fixed or variable and tailored or untailored) and findings in each of the three outcome 
categories. Non-significant findings were noted for fixed/variable interventions and health status utilization outcomes $\left(\chi^{2}{ }_{(1)}=1.691, p=0.193\right)$, health status $\left(\chi_{(1)}^{2}=1.527, p=0.217\right)$, and selfrated outcomes $\left(\chi^{2}{ }_{(1)}=0.000, p=1.000\right)$. Non-significant findings were also noted for tailored/untailored interventions and health status utilization outcomes $\left(\chi^{2}(1)=1.691, p=0.193\right)$, health status $\left(\chi^{2}{ }_{(1)}=2.857, p=0.091\right)$, and self-rated outcomes $\left(\chi^{2}(1)=0.686, p=0.408\right)$. Please see Appendix Q for all chi-square results.

Approach. The approach of interventions was reported in all $(n=46,100 \%)$ of the interventions included in this review. In terms of health service utilization outcomes, significant findings were identified in four $(8.7 \%)$ interventions, and variable in one $(2.2 \%)$ of those using a standardized approach to intervention implementation; non-significance was reported in seven $(15.2 \%)$ of these interventions. Those using a tailored approach yielded significant findings in one $(2.2 \%)$ intervention and non-significant findings in one $(2.2 \%)$. A combination of standardized and tailored treatment strategies yielded significance in seven (15.2\%) interventions and non-significance in two $(4.3 \%)$ interventions.

In terms of health status outcomes, significant and variable results were yielded in $4.3 \%$ $(n=2)$ of articles employing a standardized approach to intervention implementation; nonsignificance was reported in seven $(15.2 \%)$ of these interventions. Using a tailored approach yielded significant findings in one (2.2\%) intervention, non-significant findings in three (6.5\%), and variable results in one $(2.2 \%)$. A combination of standardized and tailored intervention strategies yielded significance in two (4.3\%) of interventions, non-significant findings were also identified in two $(4.3 \%)$, and variable in three $(6.5 \%)$.

In relation to self-rated outcomes, significance was yielded in four $(8.7 \%)$ of interventions using a standardized approach to intervention implementation; non-significance 
was also reported four $(8.7 \%)$ of these interventions. Treatments which used a tailored approach yielded significant findings in one $(2.2 \%)$ intervention and non-significant findings in zero (0\%). A combination of standardized and tailored strategies yielded significant findings in three (6.7\%) interventions and non-significance in one $(2.2 \%)$.

A chi-square test was used to assess for a relationship between the approach to which the intervention was implemented (standardized, tailored, or variable) and findings for each of the three outcome categories. Non-significant findings were noted for the approach to intervention delivery and health service utilization $\left(\chi^{2}{ }_{(2)}=3.443, p=0.179\right)$, health status utilization outcomes $\left(\chi 2_{(2)}=1.078, p=0.583\right)$, and self-rated outcomes $\left(\chi_{(2)}=1.381, p=0.501\right)$. Please see Appendix Q for all chi-square results. 


\section{Chapter Six}

\section{Discussion}

The following chapter included a description of the study findings. The primary objective of the current systematic review was to examine the intervention characteristics (component, mode, dose, and approach) of the transition-based literature and to explore the effects of these characteristics on a variety of quality of care measures. Firstly, the characteristics of the primary research studies were discussed, including descriptions of the methodological quality, study design, and sample population. Secondly, descriptions of the intervention characteristics were provided. Lastly, insights into the findings of this systematic review were described in detail.

\section{Characteristics of Included Studies}

The majority $(\mathrm{n}=17)$ of studies were of strong methodological quality based on the Quality Assessment Tool for Quantitative Studies, developed by the Effective Public Health Practice Project (EPHPP). Moderate $(n=12)$ and low $(n=9)$ quality ratings were commonly due to lack of blinding and/or selection bias. Failure to blind outcome assessors of the intervention or failing to blind participants of the research question were commonly reported amongst studies. Additionally, most studies included participants who were referred based on the acute care unit they were admitted to, leading to potential selection bias.

\section{Theory, Models, and Frameworks}

As asserted by Sidani and Braden (1998), theory should guide intervention planning, interpretation, and application of findings in the clinical setting. Interventions grounded in theory may be more effective in producing desired effects, especially when considering aspects 
of behaviour modification, such as nutritional/exercise changes, fall-prevention techniques, and adaptation strategies, often targeted in the transition-based literature (Chinn \& Kramer, 2015; Sidani \& Braden, 2011). Furthermore, the deliberative use of a validated theoretical framework can provide further insight into the soundness of theoretical relationships, and can ensure that interventions are appropriate, effective, and safe to deliver (Sidani \& Braden, 2011). With that said, the theoretical knowledge embedded within middle range theories can provide insight into the most appropriate component(s), mode, dose, and approach to be given to achieve the desired effects (Sidani \& Braden, 2011). Therefore, during the examination of the characteristics of the transition-based literature, it is important to note whether relevant practical theory was considered.

The use of a theoretical framework or model was inconsistent across studies. Factors known to influence health behaviour, such as theories of self-determination and selfmanagement, were discussed in three studies (Chow \&Wong, 2014; Ekelund \& Ekelund, 2015; Wong, Ho, Yeung, Tam, \& Chow (2011)). Broader frameworks, such as those mentioned above, were used to guide the design of intervention components aimed to modify individual behaviours or enhance self-efficacy. For example, the study by Wong et al. (2011) used Andersen's model to explain behaviours associated with increased hospital utilization. The model described three concepts that may influence one's use of health services, including predisposing factors (age, gender, ethnic group, socioeconomic status), enabling factors (supportive resources, such as intervention programs), and need factors (patient/caregiver subjective assessment of health condition/needs). The correlation between these concepts and health service utilization guided the development of strategic intervention components aimed to target each factor. 
Explicit transitional care middle-range theories, such as the transitional care model (Naylor et al., 2004) and the care transitions intervention (Coleman et al., 2006) were only considered in three studies (Koehler et al., 2009; Parry, Min, Chugh, Chalmers, \& Coleman, 2009; Xueye, Hao, Shunlin, Rongbin, Yuan, 2017). Both models were designed to support the transitional care process, beginning with in-patient hospital visits and continuing with home visits several weeks post-discharge (Coleman et al., 2006; Naylor et al., 2004). Additionally, both models incorporated face-to-face home visits, telephone follow-up, and inpatient/outpatient collaboration, to improve the quality of transitions. The transitional care model depicts an innovative care process specifically designed for high risk seniors transitioning from acute care to home (Naylor et al., 2004). Within this model, specially trained advanced practice nurses deliver a variety of transition-based care, such as symptom management, preparing seniors and caregivers to manage care at home, and education practices (Naylor et al., 2004). The care transitions intervention consists of four pillars: (1) medication self-management; (2) use of a patient-centered record; (3) follow-up care; and (4) knowledge of symptoms and indicators of worsening health conditions (Coleman et al., 2006; Parry, Coleman, Smith, Frank, \& Kramer, 2003). Substantial research on both models have demonstrated efficacy in reducing health care utilization measures (i.e. health care costs and readmission rates), health status outcomes (i.e. physical function), and self-rated outcomes (i.e. quality of life, satisfaction with care) (Naylor et al., 2004; Parry et al., 2003).

Notably, each theory-based study indicated statistically significant results in at least one of the outcomes being studied. More specifically, of the ten studies that used a framework, model, or theory to guide the intervention design/implementation process, six indicated significant results and four indicated mixed findings. This may be an indication that the use of 
empirically validated theories may provide comprehensive rationale into the most effective intervention strategies, mode of delivery, amount, frequency, and duration of treatments, and approach to implementation.

This study conceptualized the components of transition-based interventions (supportive care, educational, medication management, organizational) based on the ten domains described in the ideal transition in care model by Burke et al. (2013). The model suggests that optimal transitional care requires most, if not all, of the domains to be addressed. When considering the interventions included in this review, multicomponent interventions that included a variety of transitional care strategies (e.g. supportive care follow-up and medication safety) were more likely to address several of these domains. Additionally, studies guided by similar theories, such as the care transitions intervention model, were more likely to adhere to multiple domains of transition-based care. Lastly, as Burke's model describes both pre-discharge care (e.g. discharge planning) and post-discharge follow-up (e.g. monitoring and managing symptoms), interventions which started in the acute care setting and continued in the senior's home were more likely to address the range of strategies detailed in the ideal transition in care model. The descriptive findings of this review suggest that each of these factors (multicomponent interventions, theorydriven interventions, the inclusion of both pre-and-post discharge care strategies) may be associated with enhanced quality of care.

\section{Description of the Sample}

This study considered a sample population of seniors aged 65 years and older. Within the 38 studies included in this review, the average age of participants in the control and experimental group was roughly 80 years of age. The literature suggests that older seniors (age 85 and older) may differ from younger seniors (age 65-84) in terms of physical, mental, and functional status. 
For instance, as age increases, the prevalence of chronic comorbid conditions, physical and function decline, and cognitive deficits, also increases (CIHI, 2011). This may be an area for concern, as an increase in complexity of care may require alternative treatment strategies. The potential variation that exists between young and old seniors should be considered when designing, implementing, and evaluating transition-based interventions. As such, significant interventions may not be relevant to older seniors; further research examining treatments designed specifically for older seniors ( 85 and older) may be warranted.

Conceptualization of the term 'senior' is a topic of controversy amongst the literature (CIHI, 2011). While a single definition, such as chronological age, is commonly used by researchers, other markers, such as social, cultural, and/or functional indicators have been deemed appropriate (CIHI, 2011). Aspects such as one's level of frailty, number of chronic illnesses, and change in social role/capabilities have been suggested as suitable indicators of old age (Allen et al., 2014; World Health Organization, 2018). Although each study included in this review used chronological age as the primary parameter, several studies also used physical, functional, and/or cognitive determinants of old age during their selection process. Studies further defined the sample population in relation to the following: the number of chronic comorbid conditions (Basger, Moles, \& Chen, 2015; Berglund, Hasson, Kjellgren, \& Wilhelmson, 2015; Berglund et al., 2013; Courtney et al., 2009; Ebrahimi, Eklund, DahlinIvanoff, Jakobsson, \& Wilhelmson, 2017; Ekelund \& Eklund, 2015; Eklund et al., 2013; Finlayson et al., 2018; Chow \& Wong, 2014; Koehler et al., 2009; Kwok, Lee, Woo, Lee, \& Grffith, 2008; Stevens-Lapsley et al., 2016; Yu et al., 2015; Xueyu Li et al., 2015), level of dependence regarding activities of daily living (Berglund et al., 2013; Berglund et al., 2015; Ebrahimi et al., 2017; Ekelund \& Eklund, 2015; Eklund et al., 2013; Finlayson et al., 2018; 
Koehler et al., 2009; Stevens-Lapsley et al., 2016), risk of falls (Altfeld et al., 2013; Ueda et al., 2017), risk of malnutrition (Beck et al., 2013; Lindegaard Pedersen, Pedersen, Damsgaard, 2017; Pedersen, Pedersen, Damsgaard, 2016), and/or number of prescription medications (Altfeld et al., 2013; Koehler et al., 2009), in addition to chronological age.

As stated, this study set no limitations based on level of cognition. With that said, most primary studies required participants to undergo a mini-mental examination prior to acceptance into the study. Participants suffering from cognitive deficits, severe psychiatric conditions, or progressive neurological disease were often excluded. Eliminating these individuals creates a more homogenous sample which accounts for potential confounding variables. While this approach provided important insights and strengthened internal validity, it may not provide a true representation of the senior population. Therefore, although several studies yielded statistical significance in a variety of quality of care indicators, there may be barriers to their adoption in clinical settings due to difficulty tailoring intervention strategies to meet the needs of patients with cognitive deficits.

\section{Description of the Interventions}

The interventions included in this study shared broadly similar transitional care strategies. Most interventions were initiated in the acute care setting and continued into the home environment. Variation was noted in the timing of the initial contact, as some interventions ( $\mathrm{n}=$

5) began early in the acute care phase, such as in the emergency department setting (Berglund et al., 2014; Brovold et al., 2013; Ebrahimi et al., 2017; Eklelund \& Eklund, 2015; Eklund et al., 2013), while others $(n=20)$ began during the admission phase or prior to discharge from the inpatient unit (Beck et al., 2015; Basger et al., 2015; Beelen et al., 2017; Clemson et al., 2016; Courtney et al., 2018; Courtney et al., 2009; Courtney et al., 2012; DiMonaco et al., 2015; Chow 
\& Wong, 2014; Koehler et al., 2009; Kwok et al., 2008; Li Xueyu et al., 2017; Parry et al., 2009; Rasmussen et al., 2016; Saleh et al., 2012; Ueda et al., 2017; Wong et al., 2014; Yu et al., 2015). Few studies $(\mathrm{n}=21)$ described interventions that were initiated in the home care setting, directly following a discharge from the hospital (Aguado et al., 2010; Altfeld et al., 2013; Beck et al., 2013; Finlayson et al, 2018; Gurwitz et al., 2014; Haines et al., 2009; Lindegaard Pederson et al., 2017; Pederson et al., 2016; Rytter et al., 2010; Stevens-Lapsley et al., 2016; Thygesen et al., 2015; Toye et al., 2016; Vogler et al., 2012; Xueyu Li et al., 2015). When examining the characteristics of interventions, the timing of treatments can impact the specific components the intervention is comprised of, the mode of delivery, and the frequency and duration of implementation. The variation noted in the timing of interventions may be indicative of a lack of understanding regarding the most effective transitional care strategies and dose measures.

The majority ( $\mathrm{n}=35,76.1 \%)$ of interventions comprised of multiple care components, with the most common being a combination of supportive care, educational, and/or organizational aspects of care. Notably, each multicomponent intervention reported continuity of care strategies, such as communication between inpatient and outpatient providers, follow-up in the community, and/or assessment of symptoms post-discharge. Almost every intervention $(\mathrm{n}=38,82.6 \%)$ included a face-to-face visit pre-and/or-post-discharge, with some interventions $(\mathrm{n}=12,26 \%)$ supplementing telephone contacts between visits. Please see appendix I for descriptions of each component type and appendix $\mathrm{H}$ for a breakdown of each intervention characteristic.

This study did not yield statistically significant findings in relation to the number and type of components, or the mode of treatment delivery. However, findings from the descriptive analysis suggest a relationship between multi-component interventions which include both pre- 
and-post discharge visits, and improved quality of care. The findings from this review are similar to those yielded by Mora et al. (2017) and Kirk (2014), which reported that interventions which include follow-up phone calls and in-person home visits may be more effective at improving quality of care (e.g. readmission rates). Additionally, similar to the findings of this review, previous literature suggests that multicomponent interventions are more likely to produce statistically significant changes in quality of care (e.g. readmission rates, quality of life, mortality rates, self-rated outcomes) (Burke et al., 2013; Hsaio-mei et al., 2017; Kim \& Thyer, 2015; Kirk, 2014; Le Berre et al., 2017; Mora et al., 2017; Naylor et al., 2004).

Interventions were commonly delivered solely to patients $(n=28,60.9 \%)$; however, 17 interventions included the patient's family caregiver in the treatment strategies. From a practical perspective, healthcare providers may not have sufficient time or resources to adequately liaise

with family caregivers during the discharge planning process (Morrow \& Nicholson, 2016; Toye et al., 2016). This may be an important and undervalued aspect of transitional care, as previous research revealed that care providers often feel underrecognized and excluded from discharge decisions in hospital settings (Toye et al., 2016; Bridges). Additionally, caregivers often assume significant responsibilities during the transitional care process. Therefore, transitional care strategies, such as education and assessment, may be more effective with the family caregiver actively involved. Further research is needed which emphasizes a collaborative approach between informal caregivers and treatment providers during the discharge process.

\section{Descriptions of the control group}

Reports of the type of care the control group received varied among studies. Control group participants received routine discharge care practices, which varied between studies. Thirteen studies did not include descriptions of exactly what the routine discharge practices 
entailed (Aguado et al., 2010; Altfeld et al., 2013; Finlayson et al., 2018; Basger et al., 2015; Berglund et al., 2014; Di Monaco et al., 2015; Gurwitz et al., 2014; Haines et al., 2009; Kwok et al., 2008; Parry et al., 2009; Rasmussen et al., 2016; Rytter et al., 2010; Saleh et al., 2012). The remaining 33 studies included detailed descriptions of the care that the control group received, which often included discharge planning in the hospital (Chow \& Wong, 2014; Clemson et al., 2016; Courtney et al., 2012; Courtney et al., 2009; Ekelund \& Eklund, 2015; Eklund et al., 2013; Koehler et al., 2009; Lindegaard Pedersen, et al., 2017; Toye et al., 2016; Ueda et al., 2017), rehabilitation resources (Courtney et al., 2012; Courtney et al., 2009; Ekelund \& Eklund, 2015), and follow-up support in the community (Beck et al., 2013; Beelen et al., 2017; Brovold et al., 2013; Chow \& Wong, 2014; Courtney et al., 2012; Courtney et al., 2009; Ebrahimi et al., 2017; Eklund et al., 2013; Lindegaard Pedersen et al., 2017; Pederson et al., 2016; Stevens-Lapsley et al., 2016; Thygesen et al., 2015; Toye et al., 2016; Ueda et al., 2017; Vogler et al., 2012; Xueyu Li et al., 2015; Xueyu Li et al., 2017; Yu et al., 2015).

Although intervention group participants received additional treatment, the usual care strategies delivered to the control group often included similar attributes, such as pre-and-postdischarge treatments. The overlap between the programs delivered to intervention and control groups may have contributed to the lack of significant differences between intervention and control groups, possibly resulting in inaccurate conclusions. It would be difficult to eliminate issues regarding potential similarities in care, as it is unethical to remove all discharge strategies for the control groups. To address this issue, it is recommended that researchers and practitioners account for the potential overlap between groups and how this may impact the overall findings of studies. More specifically, this is an important aspect to consider when examining the relationship between intervention characteristics and treatment success. 


\section{Intervention Characteristics}

\section{Component}

Descriptions of the intervention component(s) were reported in each of the studies included in this review. Definitions and examples of each component can be found in appendix I. Studies comprised of either a single educational, medication management, or supportive care component, or a combination of two or more components. Within the literature, singleeducational interventions included falls prevention strategies (Ueda et al., 2016) and nutritionbased counselling (Pedersen et al., 2015). Medication management strategies, which were used as the sole intervention component in one study, included medication counselling, reconciliation, identification of potential drug related problems, and the transfer of the patient's medication review from inpatient to outpatient providers (Basger et al., 2015). Due to the small number of studies that were comprised of a sole educational/medication management component, it is difficult to make inferences regarding effectiveness.

Supportive care components included discharge follow-up in the community, assessment of health status post-discharge, enlisting community supports, coordinating care, and continuation of care in the community (Altfeld et al., 2012; Beelen et al., 2017; Brovold et al., 2013; Courtney et al., 2012; Finlayson et al., 2018; Li Xueyu et al., 2017; Vogler et al., 2012). The use of a single supportive care component was reported in seven studies, which evaluated health service utilization, health status, and self-rated outcomes. The results of these studies suggest that supportive care components could have an effect on health status (e.g. physical status) and self-rated outcomes (e.g. health-related quality of life), although sample sizes were small. However, most studies comprised of a single supportive care component indicated nonsignificant or variable results when considering all three types of outcomes (health service, 
health status, and self-rated outcomes) (Please see Appendix L, M, and N for a breakdown of outcomes based on each intervention characteristic).

Organizational components were operationalized as any intervention that addressed healthcare provider activities and/or which support the provision of effective, efficient, equitable, timely, safe and patient-centred transitional care during the hospital stay. Organizational components included rehabilitation programs, discharge planning summaries, and multidisciplinary coordination within the healthcare setting (Hsaio-Mei et al., 2017; Kim \& Thyer, 2015; Mora et al., 2017). It is important to note that no studies were limited to a single organizational component. Rather, organizational components were commonly used in combination with other strategies, such as supportive care or educational components. This finding is consistent with the current trend in the transitional care literature, which stresses the importance of including both pre-and-post-discharge activities. Strategies such as promoting continuity of care, enhancing inpatient-outpatient communication, and providing follow-up care at home are widely implemented in the transition-based literature (Coleman et al., 2003; Hsaiomei et al., 2017; Kim \& Thyer, 2015; Kirk, 2014; Le Berre et al., 2017; Mora et al., 2017; Naylor et al., 2011).

The limited number of studies focused on either a single organizational, medication management, and educational component may be indicative of current discharge-related practices in the hospital setting. Such strategies were commonly described as aspects of 'usual' discharge care amongst the transitional care literature (Chow \& Wong, 2014; Clemson et al., 2016; Courtney et al., 2012; Courtney et al., 2009; Ekelund \& Eklund, 2015; Eklund et al., 2013; Koehler et al., 2009; Lindegaard Pedersen, et al., 2017; Toye et al., 2016; Ueda et al., 2017). Conversely, providing follow-up support in the home may be considered a supplementary 
component of standardized transition-based care, as it requires additional time and resources outside of the acute care facility. As issues related to high levels of readmission rates and adverse events following hospital discharge continue to present prevalent systemic healthcare issues, usual care practices may need to be reevaluated (Allen et al., 2014; CIHI, 2011). Additional research may be needed which offers innovative approaches aimed to improve hospital-based patient education, medication management, and/or organizational strategies for seniors being discharged from the hospital to home.

Multi-component interventions led to statistically significant improvements in a variety of health service utilization, health status, and self-rated outcomes. Descriptive findings revealed that among the 46 interventions included in this review, 16 indicated statistical significance in each outcome under investigation. Within these 16 interventions, only one was comprised of a single component (educational), whereas the rest used a combination of components in the intervention design. More specifically, thirteen interventions used two components, with organizational and supportive care components being described in ten, and supportive care and educational strategies being described in three. With that said, the results of this study did not report statistical significance when assessing for a relationship between the number/type of components and health-service, health status, and self-rated outcomes. A lack of significance may be a result of the small sample sizes reported for certain component types (e.g. medication management, organizational). However, the findings revealed from descriptive analysis are congruent with previous transition-based reviews, which state that multi-component interventions may be more effective than single component (Hsaio-mei et al., 2017; Kim \& Thyer, 2015; Kirk, 2014; Le Berre et al., 2017; Mora et al., 2017; Naylor et al., 2011).

\section{Mode}


The majority of interventions used either a face-to-face mode of delivery $(n=16)$, or a combination of face-to-face and telephone strategies in the intervention design $(\mathrm{n}=12)$. Face-toface strategies were used during the participant's hospital admission and as a follow-up strategy in the community. In terms of the effect on health service utilization outcomes, employing a combination of delivery strategies achieved statistically significant findings in most studies ( 9 out of 11); whereas using a single delivery strategy (such as face-to-face or telephone strategies) may be less effective at improving health service utilization. With regards to self-rated outcomes, using a combination of delivery strategies also improved several outcomes (5 out of 8), specifically health-related quality of care, life satisfaction, quality of care, self-management skills and abilities, and preparedness to provide care at home (Berglund et al., 2013; Berglund, Hasson, Kjellgren, \& Wilhelmson, 2015; Courtney et al., 2009; Li Xueyu et al., 2017; Saleh, Freire, Morris-Dickinson, \& Shannon, 2012; Toye et al., 2016; Xueyu Li et al., 2015). Employing a single strategy, such as using a face-to-face or telephone follow-up, was less prevalent in the transition-based literature examining self-rated outcomes, making it difficult to make inferences.

Incorporating multiple delivery methods, such as face-to-face visits, telephone follow-up, and written strategies, may be necessary to account for the complex of care needs of the senior population. Seniors may experience health and psychosocial issues which may impede information uptake, such as hearing impairment, lack of access to a telephone, visual impairment, and language barriers (CIHI, 2011). Additionally, providing supplemental telephone follow-up between visits was a commonly used strategy among the transition-based literature. This may provide a more practical and economical solution to supportive care in the 
community. Lastly, the additional delivery methods may result in the seniors feeling more supported and less isolated during transitions (CIHI, 2011).

Interventions using face-to-face delivery, or a combination of strategies, reported mixed effects on health status outcomes. However, significant/variable findings were reported more frequently in studies that used a face-to-face delivery strategy (4 out of 9). Additionally, although only two studies used telephone contact as the sole method of delivery, outcomes did not reach significance in relation to health status outcomes. The ability to assess the patient, visibly check for warning signs and symptoms, and evaluate the living environment for possible risk factors, may be a necessary component of interventions designed to enhance health status (Beelen et al., 2017; Brovold et al., 2013; Courtney et al., 2012; Eklund et al., 2013; Li Xueyu et al., 2017; Pedersen et al., 2016; Rasmussen et al., 2016; Rytter et al., 2010; Stevens-Lapsley et al., 2016; Xueyu Li et al., 2015; Yu et al., 2015). Further research is needed to determine the exact strategies required to promote senior's health following discharge from an acute care facility.

Transition-based interventions were delivered by a wide range of treatment providers, including nurses, advanced practice nurses, physicians, socials workers, physiotherapists, occupational therapists, and dietitians. Additionally, many interventions used a multidisciplinary approach to intervention delivery. In relation to health service utilization, treatments delivered by a registered nurse were more likely to yield significant findings in a variety of indicators (e.g. readmission rates, emergency department visits) (Aguado et al., 2010; Chow et al., 2014; Finlayson et al., 2018; Kwok et al., 2008; Parry et al., 2009; Yu et al., 2015; Wong et al., 2014). Alternatively, interventions were less likely to indicate significance when led by a single disciplinary member of the healthcare team (e.g. social workers, physiotherapists), or when 
employing a multidisciplinary approach to treatment implementation. This is congruent with previous literature, which found that nurse-led, interdisciplinary interventions have proven effective in cost saving and quality improvement outcomes, specifically in relation to the senior population (Naylor, Kurtzman, Pauly, 2009; Naylor et al., 2011).

More studies reported statistically significant findings if led by the multidisciplinary team when evaluating self-rated outcomes. Similarly, although the sample size was small, nurse-led programs appeared to be effective at enhancing health-related quality of life and preparedness for caregiving duties (Xueye Li et al., 2015; Toye et al., 2016). Alternatively, no studies led by a single member of the healthcare team (e.g. social workers, were effective at enhancing self-rated outcomes. This study found statistically significant findings for self-rated outcomes (nonsignificance) and treatment providers (single discipline).

\section{Dose}

Dose measures were considered for components that involved human contact (e.g. home visits; telephone follow-up). Alternatively, dose was not considered for transitional care components that involved minimal human contact (e.g. provision of written educational materials), as dose parameters for these intervention components would be difficult to determine (Voils et al., 2012). The length of interventions varied considerably, ranging between one and twelve months, with most interventions lasting one month in duration. There were statistical differences in the number of studies with statically significant health status outcomes among the different categories of the duration of interventions. Across each outcome category (health service utilization, health status, and self-rated) descriptive analysis revealed that interventions were successful when provided over a minimum of one to three months. More specifically, twelve interventions yielded statistical significance in health service utilization; of these twelve 
studies, the majority $(n=8)$ were between one and three months in duration. Additionally, of the studies which evaluated self-rated outcomes and yielded statistical significance $(n=8)$, of which, most studies $(n=7)$ were between one and three months. These findings are similar to those found in the Mora et al., (2017) study, which reported that interventions must include follow-up for at least two months to reduce health service utilization measures (e.g. readmission rates).

Despite the statistically significant results found in this study, it is important to note that descriptions of the dose of interventions were not consistently reported across studies. For those studies which did state this measure, rationale for dose parameters were not provided. This was especially true for certain dose intervals (i.e. the amount of time the intervention lasted and the number of times the intervention occurred per month). This may raise concern related to intervention integrity and fidelity. Additionally, thirteen studies allowed treatment providers to offer supplementary support to seniors based on clinical presentation, or as requested by the participant (coded as dose heterogeneity, tailored) (Altfeld et al., 2013; Beck et al., 2013; Beck et al., 2015; Berglund et al., 2013; Berglund et al., 2015; Courtney et al., 2009; Courtney et al., 2012; Ebrahimi et al., 2017; Haines et al., 2009; Finlayson et al., 2018; Xueye Li et al., 2015; Yu et al., 2015). Providing additional intervention sessions may lead to further issues regarding fidelity/integrity. With this said, the significance noted between the duration of interventions reinforces the importance of adequately accounting for, and providing sufficient descriptions of, the dose of interventions. Determining the most effective amount, frequency, and duration of transitional care treatments may improve intervention success.

\section{Approach}

The majority of studies that evaluated health service utilization and self-rated outcomes and used a combination of standardized and tailored interventions indicated statistical 
significance. In these studies, the intervention contained individualized components based on the client's characteristics, needs, and preferences, and core topics/strategies that were delivered to all participants (Sidani, 2015). Examples of tailored treatments included individualized education practices, personalized discharge activities, and modifications to the level of caregiver/family involvement. Core topics included standardized activities, such as video tapes, pre- designed discharge resources, or standardized telephone follow-up questions. In terms of health status outcomes, results of standardized, tailored, or a combination of both strategies, were highly variable.

Most interventions were delivered using either a standardized, or a combination of standardized and tailored approaches. Fewer studies used a tailored approach when designing and implementing interventions. This may be indicative of the overall rigor associated with quantitative research. Methodological rigor requires precision in terms of study design, data collection, analysis, and reporting (Gray et al., 2017). Additionally, the production of highly standardized quantitative research requires strong internal validity and the avoidance of confounding variables (Gray et al., 2017). Although these standardized measures are often associated with high quality research, this strategy may lead to clinical trials that are not adequately designed to address individual need, making them less effective in the general population. With that said, research by Naylor et al. (2009) suggests providing flexible transitional care for seniors that is adaptable to unanticipated issues, such as financial and regulatory constraints and patient, may be more effective. Tailored treatments may be more practical and patient-centered, warranting further research in relation to transitional care for seniors (Naylor et al., 2009).

\section{Outcomes of Quality of Care}


Issues related to limited resources have led to an increased clinical emphasis on systembased outcomes, such as readmission rates, length of stay, and health care costs. However, as detailed in this systematic review, the impact of transitional care may produce both system and individual-level changes. Other measures, such as subjective self-rated outcomes (e.g. quality of life or self-rated symptoms), also merit consideration. Most of the studies included in this review were limited to health service utilization or health status outcomes. Future research should consider patient-centered objectives, such as quality of life, patient/caregiver functioning, and patient satisfaction, in addition to organization-based outcomes, to provide a more detailed picture of the impact of treatments (Allen et al., 2014). See Appendix J for information regarding the indicators of quality of care across interventions.

\section{Summary of the Effects of Intervention Characteristics on Outcomes}

Health-Service Utilization. Results from the included studies indicated that multi-component interventions using a combination of strategies, such as face-to-face and telephone follow-up, to deliver the treatment may improve health service utilization outcomes. Most studies that were effective in improving health service utilization outcomes were led by nurses or employed a multidisciplinary approach to intervention delivery. Interventions that considered the senior's family/caregiver during the implementation process may lead to improved results. Several studies effectively decreased health service utilization outcomes when delivered over a minimum of one to three months and were initiated in the hospital. Providing dose schedules that are tailored to the senior's needs may increase the likelihood of improve reliance on healthcare services. Finally, providing a combination of standardized and tailored intervention strategies may improve outcomes related to health service utilization. 
Health-Status Outcomes. The findings of this study indicated that incorporating a supportive care component in the intervention may enhance health status related outcomes. Results of the analysis of variance test suggest a statistically significant difference between the duration of interventions. Similarly, using a face-to-face follow-up method in the community yielded significant results in several studies. Implementing the intervention in collaboration with the senior's family/caregiver, over a minimum of one to three months in duration, may enhance a variety of health status outcomes. Additionally, interventions which considered both hospitaland-home-based treatments may be associated with improved health status outcomes. Lastly, providing structured and untailored dose schedules may increase the likelihood of achieved the desired health status effects.

Self-Rated Outcomes. In terms of self-rated outcomes, more studies yielded statistically significant results when delivered by either the multidisciplinary team, or a registered nurse. Multi-component interventions which included supportive care, educational, and organizational elements were more likely to achieve desired effects. Like health service and health status outcomes, studies delivered using a face-to-face delivery were more effective. Interventions delivered to both the individual and their family/caregiver were more likely to achieve the desired effects. Although the sample size was small, employing a combination of standardized and tailored intervention strategies may enhance self-rated outcomes. Dose schedules and durations ranged considerably amongst studies, making it difficult to decipher patterns between studies. However, similar to the other two outcomes (health-service and health status outcomes), interventions appeared effective when delivered over a period of one to three months and began in the hospital setting. 


\section{Chapter 7}

\section{Implications, Limitations, Conclusion}

This chapter provides insight into the implications and limitations of this systematic review. The implications were presented in relation to the contribution to clinical practice, policy, and healthcare research. Finally, a conclusion of the overall findings was provided.

\section{Implications}

\section{Practice}

The findings of this systematic review are consistent with previous research which states that the number and type of components, mode of delivery, duration of interventions, and approach to implementation, may have an impact on the effectiveness of transition-based interventions. Overall, incremental changes in how transitional care interventions are designed and implemented should be considered to allow for improvements in health service utilization, health status, and self-rated outcomes. It is suggested that treatment providers include multiple components within their interventions, such as educational, medication management, organizational, or aspects of supportive care. For example, providing comprehensive discharge instructions and follow-up strategies (e.g. assessment of health status, enlisting community support) in the home, may enhance quality of care. Additionally, using a combination of delivery strategies, such as face-to-face consultations, telephone follow-up, or written instructions, may improve quality of care for seniors during transitions (Burke et al., 2013). By including multiple delivery methods, the interventions may better account for potential health and psychosocial issues which may impede information uptake (e.g. hearing impairment, lack of 
access to a telephone, visual impairment, and language barriers) (Allen et al., 2014; CIHI, 2011; Naylor et al., 2009).

The results of this study also suggest that treatment providers should consider engaging the patient's family or caregiver in conversations related to transitional care activities (e.g. education, follow-up instructions). Research by Morrow and Nicholson (2016) suggests that healthcare providers should involve carers and families throughout the entire transitional process from hospital to home. Caregiver engagement may include a variety of strategies, such as establishing shared decision making between patients, their family members, and hospital staff, offering caregiver support, and including families/caregivers in education sessions (Morrow \& Nicholson, 2016). Additionally, the findings from this review suggested that transitional care interventions should begin in the early stages of the patient's admission and continue one to three months post discharge. The increased complexity of care that seniors often face, such as polypharmacy and multiple chronic conditions, may require additional time, resources, and support during transitions. Therefore, providing a combination of standardized treatments (e.g. medication reconciliation, discharge summaries) and tailored strategies (such as individualized education sessions based on the senior's learning needs, amount of caregiver involvement) may improve quality of care outcomes.

With the exception of setting restrictions on level of cognition (e.g. not including participants with neurological conditions), most studies were inclusive of all seniors, regardless of their medical history or reason for admission. Therefore, the findings of this study may provide important information that can be implemented in a variety of care areas, with a diverse group of patient populations. With that said, healthcare providers may need to consider the unique needs of the patient population within their clinical setting prior to implementing 
findings. Certain health conditions or risk factors, such as cardiac disease or risk of malnutrition, may require different strategies to achieve optimal transitional care. Therefore, interventions may need to be tailored to meet the specific needs of a given patient population. Clinicians should provide customized transitional care based on the senior's unique environmental, health, and social circumstances, while incorporating the information provided from this review regarding the most effective intervention characteristics (Naylor et al., 2009).

This study examined quantitative studies that were highly standardized and controlled. Interventions often included multiple components, strategies, and procedures within their design. For example, most studies incorporated pre-and-post-discharge treatments using a face-to-face mode of delivery. Although proven successful, these interventions were often intensive and demanding on both patients and healthcare providers. Applying such practices into largely overpopulated and understaff health systems or community settings may have financial and timerelated restraints. This may be an important aspect to consider when implementing transitionbased interventions into the practice setting, as clinicians may need to garner support and use a systematic approach to implement best practice procedures. Existing research suggests several strategies, such as including the patient's family/caregiver throughout the process, enlisting the help from community supports, timely follow-up in the community, and improved interprofessional communication between inpatient and outpatient providers (Burke et al., 2013; Morrow \& Nicholson, 2016; Naylor et al., 2000; Naylor, 2012).

\section{Policy}

Providing high-quality coordination and planning of care during the transition from acute care to home may alleviate the economic burden on acute care and address individual needs. A lack of standardization of transitional care interventions was noted amongst the included 
literature. More specifically, variation in the characteristics of transitions-interventions, including the component, mode, dose, and approach, was observed. This systematic review offered detailed information into the characteristics of transition-based literature most associated with improved quality of care. Policies to support evidence-informed practice by endorsing the specific findings (e.g. providing multicomponent interventions and treatments for a minimum of one to three months) in this study are suggested as followed.

To date, the transitional care process for senior patients being discharged from acute care to home remains highly unstandardized in Canada. Although protocols and discharge procedures exist, there is no universally accepted way transitional care is delivered. This systematic review contributes to the current body of knowledge by providing addition insight into the most appropriate manner to structure, develop, and implement transitional care. For example, this study found statically significant differences in health status outcomes among the different categories of durations. Based on these findings and the results of similar studies, it is suggested that clinicians provide treatments over a minimum of one to three months, to improve health status outcomes (e.g. mortality rate, nutritional status) (Le Berre et al., 2017; Mora et al., 2017). Alleviating these potential risks may improve the overall physical and functional status of seniors, allowing them to remain safely in their home. Additionally, improving seniors' health may avoid issues related to health service utilization, such as prolonged hospital length of stay and unplanned readmission rates. Adopting innovative policies based on new evidence is especially important, as system-and- patient-related issues continue to be problematic, and the number of seniors continues to rise (CIHI, 2017).

\section{Research}


From a research perspective, further investigation into the characteristics of transitional care interventions is needed. Researchers should consider the impact of these characteristics during the design, implementation, and evaluation process. Rationale for each characteristic, including component (number and type), mode of delivery, dose, and approach of interventions, should be included in the research report. Randomized controlled trials that incorporate strategies to minimize bias due to selection and blinding will enhance the quality of the transition-based literature (Gray et al., 2017). Providing a random sample and ensuring that participants and outcome assessors are blinded to the study objective may help to address these issues (Gray et al., 2017). Appropriate dependent variables that address program goals and consider both patient-and-organizational outcomes should be selected.

The findings from this study suggest that incorporating a theoretical perspective into the design and implementation of transition-based studies may increase the likelihood of achieving desired results. Using a model, such as the ideal transition in care model proposed by Burke et al. (2013), will better guide the development of complex and multifaceted interventions and provide an improved means for interpreting the findings. For example, the results of this systematic review, as well as previous studies, suggests that multicomponent interventions may be more effective in improving quality of care for seniors (Hsaio-mei et al., 2017; Kim \& Thyer, 2015; Kirk, 2014; Le Berre et al., 2017; Mora et al., 2017; Naylor et al., 1994; Naylor et al., 1999; Naylor et al., 2004). The ten domains of the ideal transition in care model, such as advanced care planning, medication safety, and enlisting community supports, can be used as a framework to design these components (Burke et al., 2013). The results of this systematic review can also be used as a guide for how to deliver Burke's domains of transitional care (faceto-face, written, telephone), how long the intervention should last (minimum of three months), 
who should provide the treatment (nurse and multidisciplinary team), and how to deliver the intervention (standardized and tailored strategies). Overall, a more thorough understanding of each intervention characteristic can help ensure each of Burke's domains of transitional care are implemented in the most effective manner.

\section{Limitations}

The current systematic review has several limitations. First, this study was limited to quantitative research studies, including randomized controlled trials and pilot studies. Limiting the included studies to one methodology was a strategic decision, as comparing studies with different methodologies can weaken the quality of the research evidence (Gray et al., 2017). With that said, the inclusion of nonrandomized studies may have offered a complementary, more practical perspective, as such studies often validate that interventions will work under more realistic circumstances (Harden \& Thomas, 2005). The inclusion of qualitative methodologies may have offered further insight into self-rated outcomes, such as patient and caregiver perceptions of care, leading to a more detailed picture of the impact of a given transitional care program.

Another limitation is noted in the fact that some measures of quality of care were not commonly used among studies. Certain indicators, such as fear of falling, drug related problems, and perception of abilities, were less prevalent amongst the literature, whereas readmission rates, visits to primary/emergency care, and quality of life were commonly reported outcomes. In an attempt to compare study results, outcomes were grouped into three distinct categories: health service utilization, health status, and self-rated outcomes. Although this strategy allowed for meaningful descriptive and statistical analysis, many of these outcomes were vastly different 
from one another. Therefore, generalizing outcomes and comparing results could lead to a compromised analysis.

In addition to the variety noted in the outcome categories, many of the articles included in this review varied considerably in the type of intervention strategies they used, making it challenging to compare results between studies. For example, treatments which included any teaching-based strategies (e.g. falls prevention strategies, nutrition-based counselling, discharge teaching) were conceptualized as educational in nature. Additionally, supportive care included a broad range of strategies, such as follow-up in the community and coordination of care (please see Appendix I for a full breakdown of each subtype. The heterogeneity between treatment strategies resulted in the inability to perform a meta-analysis and may decrease the overall validity of the results.

Although each intervention included information pertaining the type of component(s) within the interventions and the mode of treatment delivery (face-to-face, written, telephone), certain subgroups of these characteristics were less frequently reported. The majority of interventions used a combination of components and delivery strategies, making it difficult make inferences on less frequently reported strategies. For example, few studies used a single medication management component or only included written discharge instructions. Additionally, other characteristics, such as measures of dose intervals (amount, frequency, and duration), were not commonly reported in the literature. This restricted the ability to comprehensively compare the true impact of these intervention characteristics on the outcomes. This limitation may have also contributed to the fact that this study did not, for the most part, yield statistically significant results. 
As mentioned, some characteristics (e.g. single- educational component interventions) and outcome categories (e.g. variable findings) were less frequently reported. Due to small sample size, interventions with variable findings were excluded from the chi-square analysis. This was necessary in order to meet the assumptions of the chi-square test of independence, specifically that no cell can have an expected count of less than one (Gray et al.,2017).

Additionally, the overall small sample size included in this study may have also increased the risk of making a type II error for the remainder of characteristics and outcome categories. Lastly, due to small sample sizes, the findings of this review were predominantly descriptive. Therefore, many of the results of this systematic review cannot be used to correlate variables or determine the true cause and effect between certain characteristics and outcomes.

Another limitation is noted in the inclusion of low methodological studies. Although the majority of studies were of strong or moderate quality rating, nine studies included in this review received weak ratings. Issues related to selection bias and/or lack of blinding may reduce the overall quality of the results of this study. Additionally, a limitation is noted in the lack of studies which were conducted in Canada. This may limit the feasibility and applicability of these findings to the Canadian health care system. Lastly, many primary studies were short in duration, offering follow-up periods of less than two months post-discharge. Adverse reactions, such as mortality, readmission to acute care, and decrease in quality of life, may require longterm observations to determine the true impact (positive or negative) that transitional care may have.

\section{Conclusion}

This systematic review provided detailed insight into the characteristics of transitionbased interventions most associated with improved quality of care. To the authors knowledge, 
this is the first study to review each characteristic, including the number and type of components, the mode of delivery, timing of interventions, and approach to implementation. The evidence yielded from this review supports the provision of multi-component interventions, the incorporating face-to-face follow-up in the community, and interventions led by registered nurses and/or the multidisciplinary care team. Dose parameters, specifically the duration of interventions, may have an effect on health status outcomes. Thus, providing rationale for each dose measure may enhance the validity of intervention research. This study also revealed that collaborating with the family/caregiver may improve the likelihood of achieving the desired effects. Finally, providing a combination of standardized and tailored transitional care strategies into the intervention design may enhance health service and self-rated outcomes.

Although findings were inconclusive in relation to certain characteristics and outcome measures, the results of this study justify the need for further investigation into the underpinnings of transition-based literature. Sustained emphasis on developing highly thought-out transitional care programs for seniors is imperative, as the senior population continues to increase and the demand on healthcare services subsequently rises. A more thorough understanding of the characteristics of complex transition-based interventions may lead to improved research standards and enhanced quality of care for seniors. 


\section{Appendix A: Literature Review Summary}

\begin{tabular}{|c|c|c|c|c|c|}
\hline Title & $\begin{array}{l}\text { Sample } \\
\text { Population }\end{array}$ & $\begin{array}{l}\text { TC } \\
\text { Intervention(s) }\end{array}$ & $\begin{array}{l}\text { TC Intervention } \\
\text { Characteristics }\end{array}$ & $\begin{array}{l}\text { Outcomes } \\
\text { Measures }\end{array}$ & Results \\
\hline $\begin{array}{l}\text { Hsiao-Mei, C., Yi-Hsuan, T., \& } \\
\text { Ching-Min, C. (2017). Effect of } \\
\text { Continuity of Care on Quality of } \\
\text { Life in Older Adults With Chronic } \\
\text { Diseases: A Meta-Analysis. Clinical } \\
\text { Nursing Research, 26(3), 266-284. } \\
\text { doi:10.1177/1054773815625467 }\end{array}$ & $\begin{array}{l}\text { Total Number of } \\
\text { Studies: } 7 \text { RCTs. } \\
\text { Total \# of } \\
\text { participants across } \\
\text { studies: } 1,394 \\
\text { Criteria for } \\
\text { inclusion: } \\
\text { RCT with a } \\
\text { controlled group } \\
\text { design } \\
\text { and a study } \\
\text { population of } \\
\text { adults aged } 65 \\
\text { years and older } \\
\text { with chronic } \\
\text { diseases. }\end{array}$ & $\begin{array}{l}\text { Medical } \\
\text { consultations, } \\
\text { rehabilitation } \\
\text { programs, home } \\
\text { visits, telephone } \\
\text { interviews, and a } \\
\text { detailed treatment } \\
\text { summary covered } \\
\text { in the DPSs. } \\
\text { Treatment } \\
\text { Providers: Case } \\
\text { managers could } \\
\text { be physicians, } \\
\text { nurses, social } \\
\text { workers, physical } \\
\text { therapists, } \\
\text { occupational } \\
\text { therapists, } \\
\text { dieticians, and } \\
\text { volunteers. }\end{array}$ & $\begin{array}{l}\text { Component: \# } \\
\text { and type: this } \\
\text { study found no } \\
\text { statistically } \\
\text { significant } \\
\text { findings in } \\
\text { studies that only } \\
\text { included a single } \\
\text { component. } \\
\text { Duration: } \\
\text { follow-up } \\
\text { duration ranged } \\
\text { from } 3 \text { months- } \\
\text { 12 months. } \\
\text { Frequency: Not } \\
\text { in the discussion } \\
\text { section. } \\
\text { Dose of } \\
\text { interventions } \\
\text { was not } \\
\text { considered in } \\
\text { results section. }\end{array}$ & $\begin{array}{l}\text { Quality of Life: } \\
\text { Physical } \\
\text { functioning } \\
\text { Role functioning } \\
\text { physical } \\
\text { Bodily pain } \\
\text { General health } \\
\text { Mental health } \\
\text { Role functioning } \\
\text { emotional } \\
\text { Social } \\
\text { functioning } \\
\text { Vitality }\end{array}$ & $\begin{array}{l}\text { The results } \\
\text { indicated that } \\
\text { CoC } \\
\text { intervention } \\
\text { can significantly } \\
\text { improve } \\
\text { physical } \\
\text { function, } \\
\text { physical role } \\
\text { function, } \\
\text { general } \\
\text { health, social } \\
\text { function, and } \\
\text { vitality } \\
\text { Lack of change } \\
\text { in mental health } \\
\text { and role } \\
\text { limitations }\end{array}$ \\
\hline $\begin{array}{l}\text { Kim, H., \& Thyer, B. A. (2015). } \\
\text { Does transitional care prevent older } \\
\text { adults from rehospitalization? A } \\
\text { review. Journal of Evidence- } \\
\text { Informed Social Work, 12(3), 261- } \\
271 . \\
\text { doi:10.1080/15433714.2013.827140 }\end{array}$ & $\begin{array}{l}\text { Total Number of } \\
\text { Studies: } 9 \text { RCTs. } \\
\text { Criteria for } \\
\text { Inclusion: } 65 \text { and } \\
\text { over. Inpatient to } \\
\text { outpatient } \\
\text { transitions- } \\
\text { hospital to home. }\end{array}$ & $\begin{array}{l}\text { Intensive } \\
\text { individualized } \\
\text { patient education, } \\
\text { a medication } \\
\text { review, early } \\
\text { discharge } \\
\text { planning, follow- } \\
\text { up } \\
\text { through home } \\
\text { care and } \\
\text { telephone } \\
\text { contacts, } \\
\text { comprehensive } \\
\text { discharge } \\
\text { planning, } \\
\text { medication } \\
\text { counseling } \\
\\
\text { Treatment } \\
\text { Providers: } \\
\text { Geriatric } \\
\text { cardiologist, } \\
\text { nurses, and } \\
\text { social workers }\end{array}$ & $\begin{array}{l}\text { Component: } \\
\text { Number and type } \\
\text { of intervention } \\
\text { components } \\
\text { were compared } \\
\text { between studies } \\
\text { Dose (duration): } \\
\text { considered in the } \\
\text { results section, } \\
\text { as the duration of } \\
\text { the studies varied } \\
\text { considerably (6 } \\
\text { months- one } \\
\text { year). }\end{array}$ & $\begin{array}{l}\text { Rehospitalization } \\
\text { rates. }\end{array}$ & $\begin{array}{l}7 / 9 \text { studies } \\
\text { detected the } \\
\text { positive effects } \\
\text { of } \mathrm{TC} \text { in } \\
\text { preventing } \\
\text { rehospitalization } \\
\text { rates }\end{array}$ \\
\hline $\begin{array}{l}\text { Kirk, C. (2014). telephone follow- } \\
\text { up of older people after hospital } \\
\text { admissions. Age and Ageing, } \\
\text { 43(suppl 2), ii7-ii7. } \\
\text { doi:10.1093/ageing/afu124.28 }\end{array}$ & $\begin{array}{l}\text { Total Number of } \\
\text { Studies: } 18 \text { studies } \\
\text { of telephone } \\
\text { follow-up (TFU)- } \\
3 / 18 \text { were TFU as } \\
\text { the sole } \\
\text { intervention. } 15 / 18 \\
\text { used TFU as a part } \\
\text { of a } \\
\text { multicomponent } \\
\text { transitional care } \\
\text { intervention }\end{array}$ & $\begin{array}{l}\text { TFU post hospital } \\
\text { discharge. } \\
\text { Includes TFU in } \\
\text { sole and } \\
\text { multicomponent } \\
\text { interventions. } \\
\text { Treatment } \\
\text { Providers: }\end{array}$ & $\begin{array}{l}\text { Mode (medium): } \\
\text { telephone } \\
\text { follow- up } \\
\text { Dose: } 24 \text { hours- } \\
72 \text { hours } \\
\text { Some studies } \\
\text { evaluated dose: } \\
\text { reporting on } \\
\text { frequency and } \\
\text { duration of } \\
\text { intervention. }\end{array}$ & $\begin{array}{l}\text { Health care } \\
\text { utilization } \\
\text { outcomes: } \\
\text { readmissions } \\
\text { rates, } \\
\text { ED/outpatient } \\
\text { clinic visits. }\end{array}$ & $\begin{array}{l}\text { Care transitions } \\
\text { that include } \\
\text { both in- hospital } \\
\text { and home } \\
\text { treatment } \\
\text { components } \\
\text { may be most } \\
\text { effective at } \\
\text { reducing } \\
\text { readmission } \\
\text { rates. } \\
\text { Discharge care } \\
\text { planning in }\end{array}$ \\
\hline
\end{tabular}




\begin{tabular}{|c|c|c|c|c|c|}
\hline & $\begin{array}{l}\text { Criteria for } \\
\text { Inclusion: }\end{array}$ & & $\begin{array}{l}\text { Reported on } \\
\text { common } \\
\text { components used } \\
\text { with TFU that } \\
\text { reduce } \\
\text { readmission, } \\
\text { including, patient } \\
\text { education, } \\
\text { educational } \\
\text { materials, home } \\
\text { visits, and } \\
\text { providing } \\
\text { follow-up (i.e. } \\
\text { contact } \\
\text { numbers). }\end{array}$ & & $\begin{array}{l}\text { hospital, patient } \\
\text { education, early } \\
\text { (within the first } \\
\text { week of } \\
\text { discharge) and } \\
\text { frequent ( } 2 \text { calls } \\
\text { in the first } \\
\text { month) TFY } \\
\text { and home visits } \\
\text { are found most } \\
\text { effective. }\end{array}$ \\
\hline $\begin{array}{l}\text { Le Berre, M., Maimon, G., Sourial, } \\
\text { N., Guériton, M., \& Vedel, I. } \\
\text { (2017). Impact of Transitional Care } \\
\text { Services for Chronically Ill Older } \\
\text { Patients: A Systematic Evidence } \\
\text { Review. Journal Of The American } \\
\text { Geriatrics Society, 65(7), 1597- } \\
\text { 1608. doi:10.1111/jgs.14828 }\end{array}$ & $\begin{array}{l}\text { Total Number of } \\
\text { Studies: } 92 \text { RCTs } \\
\text { Criteria for } \\
\text { Inclusion: Patients } \\
65 \text { and older with } \\
\text { at least one chronic } \\
\text { disease. Acute care } \\
\text { to home. }\end{array}$ & $\begin{array}{l}\text { (1) aimed at } \\
\text { providing } \\
\text { coordination and } \\
\text { continuity of } \\
\text { care; (2) pre- } \\
\text { arranged } \\
\text { structured post- } \\
\text { discharge } \\
\text { follow-up (e.g., } \\
\text { home visits, } \\
\text { phone calls); (3) } \\
\text { at } \\
\text { least one follow- } \\
\text { up starting within } \\
\text { 30-days post- } \\
\text { discharge. } \\
\text { Interventions } \\
\text { included: } \\
\text { educational, } \\
\text { multidisciplinary } \\
\text { coordination, } \\
\text { collaboration, } \\
\text { cooperation } \\
\text { components }\end{array}$ & $\begin{array}{l}\text { Dose: Duration } \\
\text { and frequency of } \\
\text { intervention } \\
\text { components was } \\
\text { considered } \\
\text { Mode: discusses } \\
\text { intervention } \\
\text { medium (face-to- } \\
\text { face, written, } \\
\text { videotape) }\end{array}$ & $\begin{array}{l}\text { All-cause } \\
\text { mortality, all- } \\
\text { cause } \\
\text { readmission, all- } \\
\text { cause ED visits, } \\
\text { all-cause } \\
\text { readmission } \\
\text { days, and QoL } \\
\text { reported at } 1,3 \text {, } \\
6,12,18 \text { and/or } \\
24 \text { months. }\end{array}$ & $\begin{array}{l}\text { Reports on } \\
\text { effective } \\
\text { components: } \\
\text { positive are seen } \\
\text { with: } \\
\text { phone calls, } \\
\text { phone } \\
\text { availabilities } \\
\text { (24/7), } \\
\text { involvement of } \\
\text { a } \\
\text { pharmacist. } \\
\text { No } \\
\text { improvements } \\
\text { with: lead by a } \\
\text { nurse, } \\
\text { medication } \\
\text { reconciliation, } \\
\text { home visits, } \\
\text { telemonitoring, } \\
\text { initial contact } \\
\text { within } \\
1 \text { week of } \\
\text { discharge. }\end{array}$ \\
\hline $\begin{array}{l}\text { Mora, K., Dorrejo, X. M., Carreon, } \\
\text { K. M., \& Butt, S. (2017). Nurse } \\
\text { practitioner-led transitional care } \\
\text { interventions: An integrative } \\
\text { review. Journal Of The American } \\
\text { Association Of Nurse Practitioners, } \\
\text { 29(12), 773-790. doi:10.1002/2327- } \\
6924.12509\end{array}$ & $\begin{array}{l}\text { Total Number of } \\
\text { Studies: } 8 \text { articles: } \\
\text { including RCTs, } \\
\text { meta-analysis, and } \\
\text { non-RCTs } \\
\text { Criteria for } \\
\text { Inclusion: }\end{array}$ & $\begin{array}{l}\text { Telephone } \\
\text { follow-up, clinic } \\
\text { visit, patient } \\
\text { education, } \\
\text { community } \\
\text { referrals, } \\
\text { medication } \\
\text { Nurse- } \\
\text { practitioner } \\
\text { provided } \\
\text { transitional care } \\
\text { interventions }\end{array}$ & $\begin{array}{l}\text { Dose (duration): } \\
\text { interventions that } \\
\text { followed patients } \\
\text { for at least } 2 \\
\text { months post } \\
\text { discharge were } \\
\text { most successful } \\
\text { at reducing } \\
\text { readmission } \\
\text { Timing: ranged } \\
\text { considerably. } \\
\text { Mode: face-to- } \\
\text { face, web-based, } \\
\text { telephone was } \\
\text { listed, yet not } \\
\text { fully considered } \\
\text { in the evaluation. }\end{array}$ & $\begin{array}{l}\text { Symptoms, } \\
\text { functional status, } \\
\text { patient } \\
\text { satisfaction, self- } \\
\text { efficacy, } \\
\text { readmission } \\
\text { rates, }\end{array}$ & $\begin{array}{l}\text { Phone calls and } \\
\text { home visits } \\
\text { made by } \\
\text { NPs have } \\
\text { demonstrated } \\
\text { decreases in } \\
\text { hospital } \\
\text { readmissions, } \\
\text { but these } \\
\text { decreases often } \\
\text { were not } \\
\text { statistically } \\
\text { significant. } \\
\\
\text { No standard } \\
\text { number of home } \\
\text { visits/calls has } \\
\text { been } \\
\text { established. }\end{array}$ \\
\hline
\end{tabular}




\section{Appendix B: PRISMA Flow Diagram}

\section{PRISMA 2009 Flow Diagram}

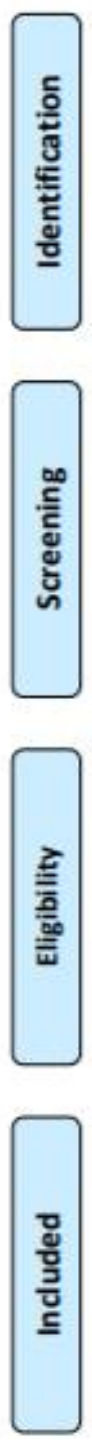

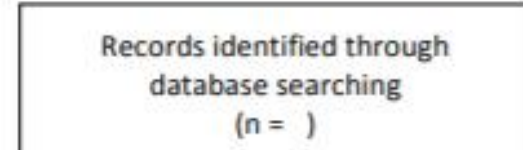

$(\mathrm{n}=)$
Additional records identified through other sources

$$
(n=)
$$

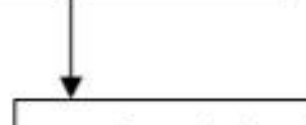

Records after duplicates removed

$$
(n=)
$$

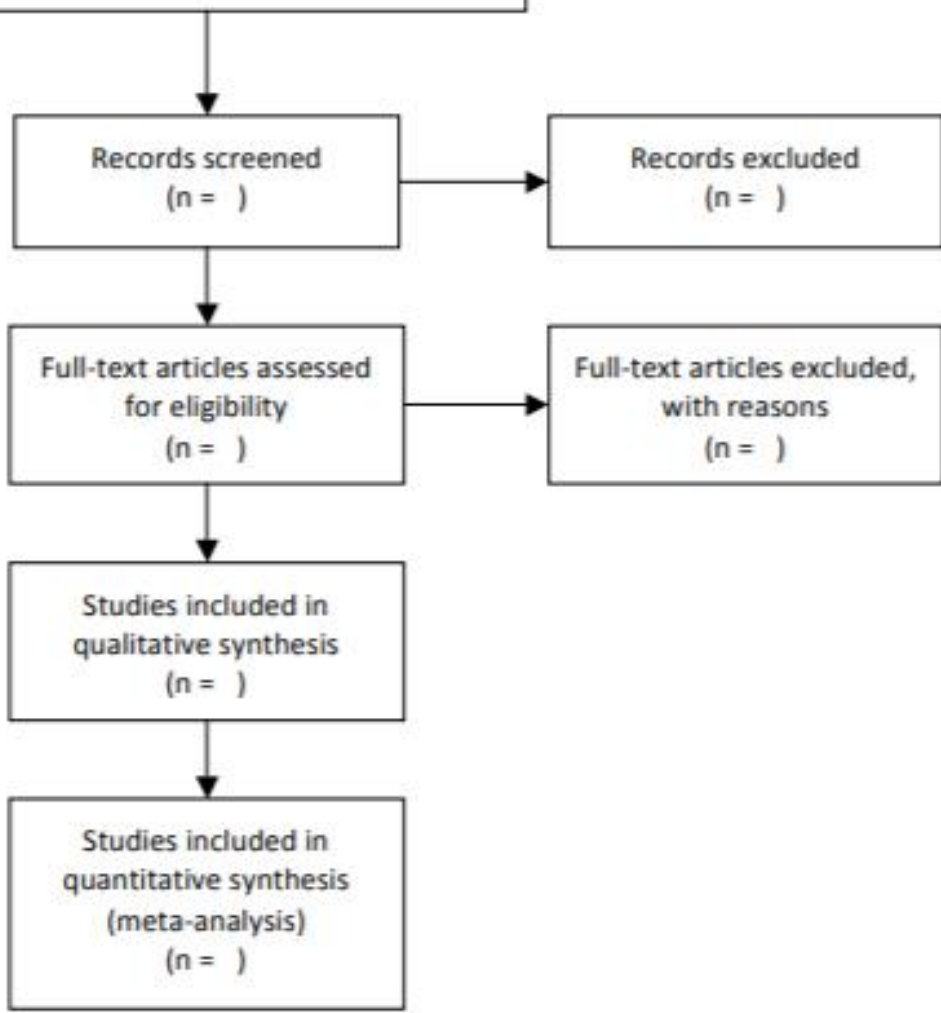




\section{Appendix C: PRISMA Checklist for Systematic Reviews}

\begin{tabular}{|c|c|c|c|}
\hline Section/Topic & litem & Checklist Hen & $\begin{array}{l}\text { Reported on } \\
\text { Page t }\end{array}$ \\
\hline \multicolumn{4}{|l|}{ TITLE } \\
\hline Tte & 1 & Identify the report as a systematic revew, meta-andysa, or both. & \\
\hline \multicolumn{4}{|l|}{ ABSTRACT } \\
\hline Structured summary & 2 & 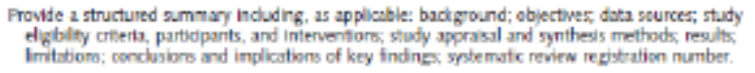 & \\
\hline \multicolumn{4}{|l|}{ INTRODUCTION } \\
\hline Rationde & 3 & Dencrbe the ntionsle for the revise in the contest of whet b atready known. & \\
\hline Objectives & 4 & 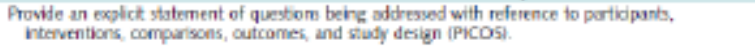 & \\
\hline \multicolumn{4}{|l|}{ METHODS } \\
\hline Protocoll and registuticn & 5 & 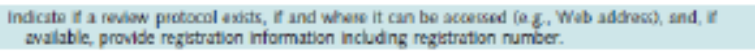 & \\
\hline Gybality critaria & 6 & 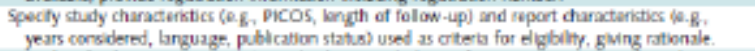 & \\
\hline Information sources & 7 & 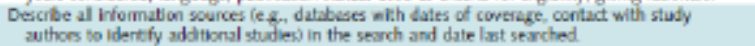 & \\
\hline Serch & $s$ & 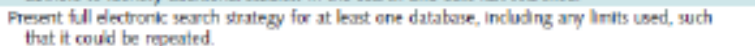 & \\
\hline Study selection & 9 & 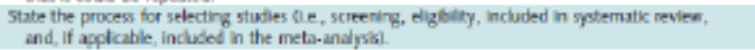 & \\
\hline Data colection process & 10 & 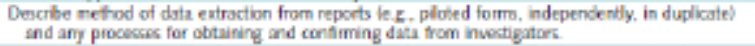 & \\
\hline Date itens & 11 & 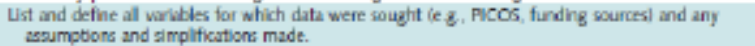 & \\
\hline $\begin{array}{l}\text { Dikk of tias in ndididual } \\
\text { studes }\end{array}$ & 12 & 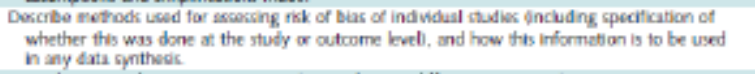 & \\
\hline Summary meavies & 13 & State the princtos sumnary measures ie $g$, isk ratio, difference in nearsed. & \\
\hline Syrthesik of recults & 14 & 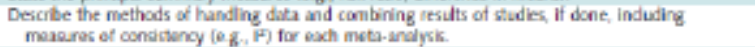 & \\
\hline $\begin{array}{l}\text { Rak of biss across } \\
\text { studes }\end{array}$ & 15 & 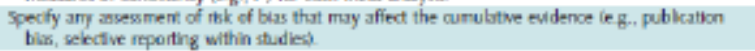 & \\
\hline Add tioned analpon & 16 & 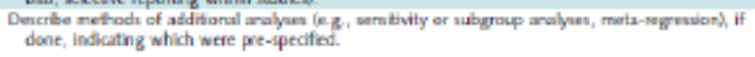 & \\
\hline \multicolumn{4}{|l|}{ RESULTS } \\
\hline Seidy selection & 17 & $\begin{array}{l}\text { Cine numbers of studes screened asesced tor elig bilty, and induded in the review, with reasons } \\
\text { for exclusions at each stage, ideally with a flow elagram. }\end{array}$ & \\
\hline Soudy charosteristics & 18 & $\begin{array}{l}\text { For each study, present cheracteristice for which dith were extracted (e g. s.udy size, Ficos, } \\
\text { follow-up period) and prowde the dtaflons. }\end{array}$ & \\
\hline $\begin{array}{l}\text { Fuak of tias within } \\
\text { studer }\end{array}$ & 19 & 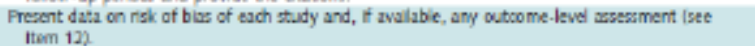 & \\
\hline $\begin{array}{l}\text { Results of individud } \\
\text { ntuden }\end{array}$ & 20 & 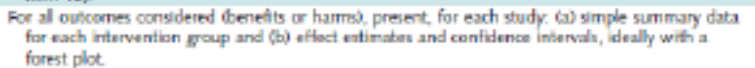 & \\
\hline Syrthesk of recults & 21 & $\begin{array}{l}\text { Fresent reafts of each meta-analyik done, including confidence intervals and mezeres of } \\
\text { corsatency. }\end{array}$ & \\
\hline $\begin{array}{l}\text { Risk of bias across } \\
\text { studes }\end{array}$ & 22 & Fresent results of anf assessment of risk of bas acoss studies tsee item 150 . & \\
\hline Addtiond anahrss & 23 & $\begin{array}{l}\text { Clve reguts of additional analyzes, if done (e } z \text {, sentivity or subgroup analyses, meta-regresson } \\
\text { [see tram } 16] \text {. }\end{array}$ & \\
\hline \multicolumn{4}{|l|}{ Discussion } \\
\hline Summary of evidence & 24 & 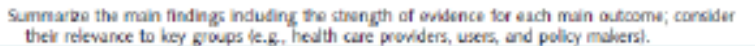 & \\
\hline Limitations & 25 & 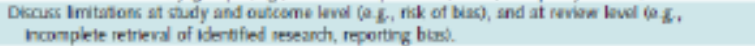 & \\
\hline Conduibions & 26 & $\begin{array}{l}\text { Provide } 2 \text { general interpetation of the reauls in the content of ether evidence, and implications } \\
\text { for future research. }\end{array}$ & \\
\hline \multicolumn{4}{|l|}{ PUNDING } \\
\hline Funding & 27 & $\begin{array}{l}\text { Describe sources of funding for the syatematic revew and other aupport (e. g-, supply of data); } \\
\text { role of funders for the systenatic revicw. }\end{array}$ & \\
\hline
\end{tabular}


Appendix D: Effective Public Health Practice Project Quality Assessment Toolf for Quantitative Studies

\section{QUALITY ASSESSMENT TOOL FOR QUANTITATIVE STUDIES}

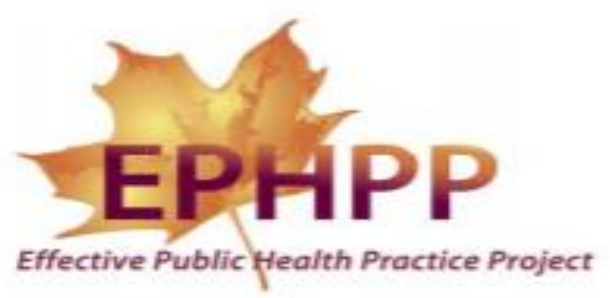

COMPONENT RATINGS

A)

SELECTION BIAS

(a1) Are the individuals selected to participate in the stady likely to be representative of the target popelation?

$$
\begin{array}{ll}
1 & \text { Very likely } \\
2 & \text { Samewhar likely } \\
3 & \text { Not likely } \\
4 & \text { Can't dell }
\end{array}
$$

(02) What percentage of selected individuals agreed to participate?

$$
\begin{aligned}
& 1 \text { 80- } 100 \% \text { agreement } \\
& 2.60-79 \% \text { agreement } \\
& 3 \text { less than 60\% agreement } \\
& 4 \text { Not applicable } \\
& 5 \text { Can't tell }
\end{aligned}
$$

$$
\text { RATE THIS SECTION }
$$

STRONG

MODerate

WEAX

See dictianary

1

2

3

B) STUDY DESIGN

Indieate the study design

1 Flandomiaed controlied trial

Controlled dinical trial

Cohort analytic (two group pre + post)

Case-contral

Cohort lone group pre + post (before and ather)

internupted time series

Other specify

a Can't tell Was the study deseribed as randomized? If NO, go to Component C.
Nos

If Yes, was the method af randomization described? (See dictionary)

No

Yes

II Yes, was the method appropriate? (See dietionary)

No

Yes

RATE THIS SECTION

See dictionary

STRONG

MOderate

WEAX

2

3 


\section{QUALITY ASSESSMENT TOOL FOR QUANTITATIVE STUDIES}

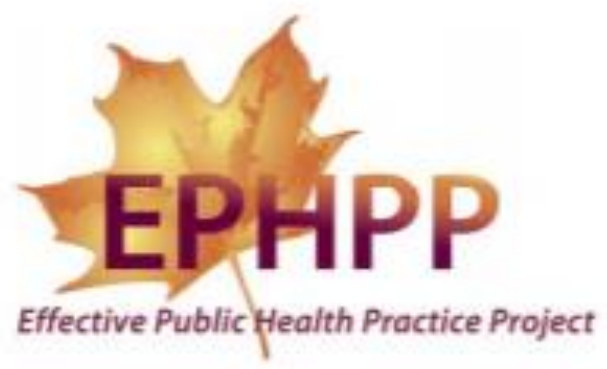

\section{COMPONENT RATINGS}

\section{A) SELECTION BIAS}

(a1) Are the individuals selected to participate in the stady likely to be representative of the target popelation?

$$
\begin{array}{ll}
1 & \text { Very likely } \\
2 & \text { Sameanhat laxely } \\
3 & \text { Not likely } \\
4 & \text { Can't dell }
\end{array}
$$

(02) What percentage of selected individeals agreed to participate?

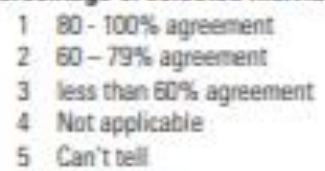

\begin{tabular}{|lccc|}
\hline RATE THIS SECTION & STRONG & MODERATE & WEAK \\
See dictianary & 1 & 2 & 3 \\
\hline
\end{tabular}

B) STUDY DESIGN

Indicate the stady design

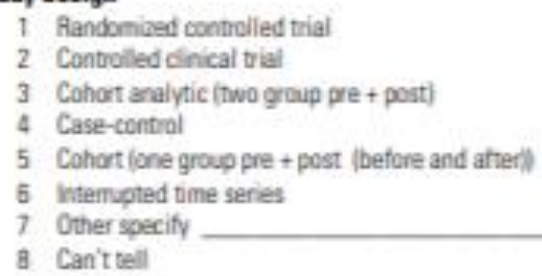

Was the study deseribed as randomized? If NO, go to Component C.

$\mathrm{No}$

Yes

If Yes, was the method af randomization described? (See dietionary)

$\mathrm{No}$

Yes

II Yes, was the method appropriate? (See dietionary)

No

Yes

\begin{tabular}{|lccc|}
\hline RATE THIS SECTION & STRONG & MODERATE & WEAX \\
See dictionary & 1 & 2 & 3 \\
\hline
\end{tabular}


C) CONFOUNDERS

(a1) Were there important differences between groups prior to the intervention?

$\begin{array}{ll}1 & \text { Yes } \\ 2 & \text { No } \\ 3 & \text { Can't tell }\end{array}$

The following are examples of contounders:

1 Race

2 Sex

3 Marital stanis/tamily

4 Age

5 SES lincome or class)

6 Escration

7 . Heain status

8 Pre-intervention scare on outcome measse

(02) If yes, indicate the percentage of relevant confounders that were cantrolled (either in the design (e.g. stratification, matching) or analysis)?
$180-100 \%$ imosti)
$260-79 \%$ (some)
3 Less than $60 \%$ ffew or nore)
4 Can't Tell

\begin{tabular}{|lccc|}
\hline RATE THIS SECTION & STRONG & MODERATE & WEAK \\
See dietioary & 1 & 2 & 3 \\
\hline
\end{tabular}

D) BLINDING

(01) Was (were) the outcome assessor(s) aware of the intervention or exposure status af participants?
1 Yes
2 No
3 Can'trel

(02) Were the study participants aware of the research question?
1 Yes
2 Na
3 Can't tell

\begin{tabular}{|lll}
\hline RATE THIS SECTION STRONG WODERATE WEA & W S
\end{tabular}

See dictioaary

1

2

E) DATA COLIECTION METHODS

(a1) Were data collection tools shown to be valid?
1 Yes
2 No
3 Can't bell

(02) Were data collection tools shown to be reliable?
1 Yes
2 No
3 Can'tel

\begin{tabular}{|lccc|}
\hline RATE THIS SECTION & STRONG & MODERATE & WEAK \\
See dictionary & 1 & 2 & 3 \\
\hline
\end{tabular}


(01) Were withdrawals and drop-outs reported in terms of numbers and/or reasons per group?

1 Yes

2. No

3 Can't tell

4 Not Applicable (i.e one tine sunvers or intenvewsi

(02) Indicate the percentage of participants campleting the study. (If the percentage differs by groups, record the lowestl.

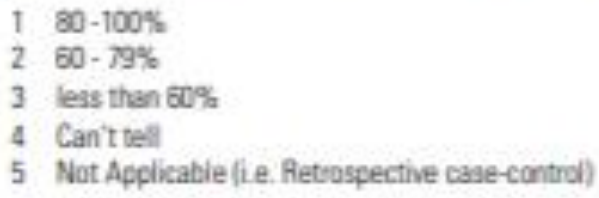

\begin{tabular}{|lcccc|}
\hline RATE THIS SECTION & STRONG & MODERATE & WEAK & \\
See dictionary & 1 & 2 & 3 & Net Apqicabie \\
\hline
\end{tabular}

G) INTERVENTION INTEGRITY

(Q1) Wat percentage of participants received the allocated intervention or exposure of interest?

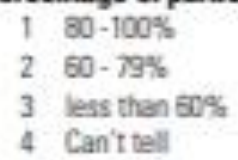

(02) Was the consisteacy of the intervention measured?

$$
\begin{array}{ll}
1 & \text { Yes } \\
2 & \text { No } \\
3 & \text { Can't tell }
\end{array}
$$

(03) Is it likely that subjects received an unintended intervention (contamination or co-intervention) that may influence the results?
4 Yes
5 No
5 Can't tell

H) ANALYSES

(a1) Indicate the unit of allocation (circle one)

$$
\text { community organivation/nstitition practice/office individual }
$$

(02) Indicate the unit of analysis (eircle ooe)
communicy organzationvinstiturtion
practicefoffice individuat

(03) Are the statistical methods appropriate for the study design?
1 Yes
2. No
3 Can't tell

(04) Is the analysis performed by intervention allocation states (i.e. intention to treat) rather than the actual intervention received?
1 Yas
2 No
3 Can'tell 
GLOBAL RATING

COMPONENT RATINGS

Please transcribe the information from the gray boxes on pages 1-4 onto this page. See dictionary on how to rate this section.

\begin{tabular}{|c|c|c|c|c|c|}
\hline \multirow[t]{2}{*}{ A } & SELECTION BIAS & STRONG & MODERATE & WEAK & \\
\hline & & 1 & 2 & 3 & \\
\hline \multirow[t]{2}{*}{ B } & STUDY DESIGN & STRONG & MODERATE & WEAK & \\
\hline & & 1 & 2 & 3 & \\
\hline \multirow[t]{2}{*}{ c } & CONFOUNDERS & STRONG & MODERATE & WEAK & \\
\hline & & 1 & 2 & 3 & \\
\hline \multirow[t]{2}{*}{ D } & BLINDING & STRONG & MODERATE & WEAK & \\
\hline & & 1 & 2 & 3 & \\
\hline \multirow[t]{2}{*}{ E } & $\begin{array}{l}\text { DATA COLLCTION } \\
\text { METHOD }\end{array}$ & STRONG & MODERATE & WEAK & \\
\hline & & 1 & 2 & 3 & \\
\hline \multirow[t]{2}{*}{$\mathbf{F}$} & $\begin{array}{l}\text { WITHDRAWALS AND } \\
\text { DROPOUTS }\end{array}$ & STRONG & MODERATE & WEAK & \\
\hline & & 1 & 2 & 3 & Not Applicable \\
\hline
\end{tabular}

\section{GLOBAL RATING FOR THIS PAPER /circle onet:}

$\begin{array}{lll}1 & \text { STRONG } & \text { (no WEAK ratings) } \\ 2 & \text { MOOERATE } & \text { (one WEAK rating) } \\ 3 & \text { WEAK } & \text { (two or more WEAK ratings) }\end{array}$

With boch reviewers discussing the ratings:

Is there a discrepancy becween the two reviewers with respect to the component (A-F) ratings?

No Yes

If yes, indicate the reasen for the discrepancy
1 Oversight
2 Differences in interpretation of criteria
3 Differences in interpretation of study

\begin{tabular}{llll} 
Final decision of both reviewers (circle one): & 1 & STRONG \\
\cline { 1 - 4 } & 2 & MODERATE \\
& 3 & WEAK
\end{tabular}




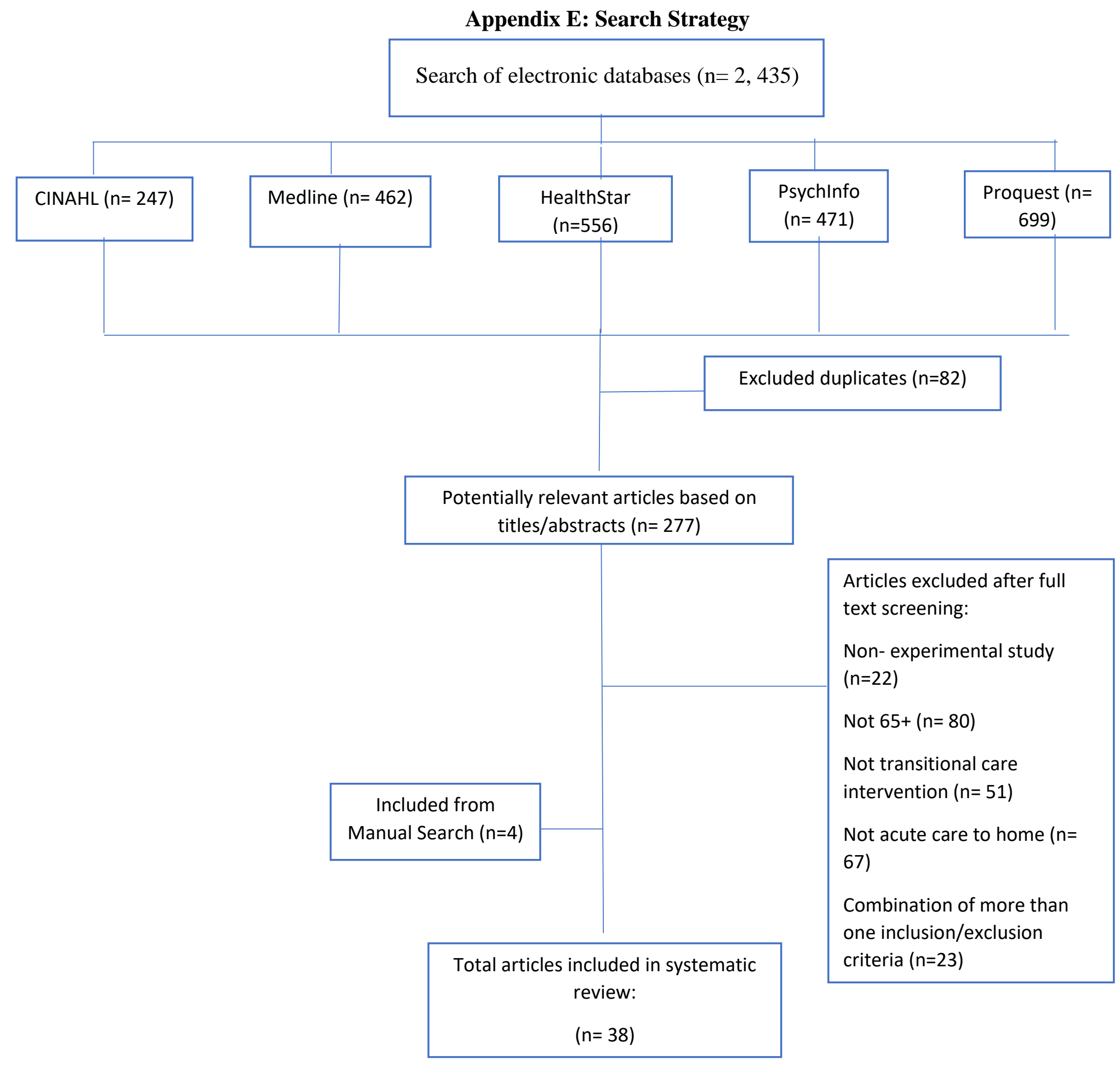




\section{Appendix F: Table of Included Studies}

\begin{tabular}{|c|c|c|c|c|c|}
\hline $\begin{array}{c}\text { Study ID, Review Author, } \\
\text { Citation, Year of } \\
\text { Publication }\end{array}$ & $\begin{array}{l}\text { Study and Sample } \\
\text { Characteristics }\end{array}$ & $\begin{array}{l}\text { Primary Outcome } \\
\text { Measures and Time } \\
\text { Points Collected }\end{array}$ & $\begin{array}{l}\text { Measurement Tools } \\
\text { and Reports of } \\
\text { Reliability/Validity }\end{array}$ & $\begin{array}{c}\text { Intervention } \\
\text { Characteristics }\end{array}$ & $\begin{array}{l}\text { Quality of Care } \\
\text { Indicators and } \\
\text { Findings }\end{array}$ \\
\hline $\begin{array}{l}\text { Study ID: } 1 \\
\text { Review Author ID: } 1 \\
\text { Citation: } \\
\text { Beck, A. M., Kjær, S., } \\
\text { Hansen, B. S., Storm, R. L., } \\
\text { Thal-Jantzen, K., \& Bitz, C. } \\
\text { (2013). Follow-up home visits } \\
\text { with registered dietitians have } \\
\text { a positive effect on the } \\
\text { functional and nutritional } \\
\text { status of geriatric medical } \\
\text { patients after discharge: A } \\
\text { randomized controlled trial. } \\
\text { Clinical Rehabilitation, } 27(6) \text {, } \\
\text { 483-493. } \\
\text { doi:10.1177/02692155124693 } \\
\text { 84 } \\
\text { Date of Study: } 2013\end{array}$ & $\begin{array}{l}\text { Purpose: To assess the } \\
\text { additional benefits of } \\
\text { individualized } \\
\text { nutritional counselling } \\
\text { by a registered dietitian } \\
\text { in geriatric patients' } \\
\text { home after discharge } \\
\text { from hospital, in } \\
\text { relation to risk of re- } \\
\text { admissions, functional } \\
\text { status, nutritional status, } \\
\text { use of social services } \\
\text { and mortality. } \\
\text { Study Design: } 3 \\
\text { Quality Rating: } 1 . \\
\text { Global Rating (for } \\
\text { weak/moderate } \\
\text { articles): } 1 ; 4 \text {. } \\
\text { N = 152 } \\
\text { Setting: } 4 \text {. } \\
\text { Country: } 3 \text {. } \\
\text { Medical Diagnosis: } 2 \text {. } \\
\text { Age: } 65+\end{array}$ & $\begin{array}{l}\text { Total number of } \\
\text { intervention groups: } 2 \\
\text { number of participants } \\
\text { allocated to each } \\
\text { intervention group: } \\
\text { Control: } 79 \\
\text { Intervention: } 73 \\
\text { Primary outcome(s) } \\
\text { and time points } \\
\text { collected and reported: } \\
\text { Readmission rates (1). } \\
\text { Between one and three } \\
\text { weeks after discharge, } \\
\text { and } 26 \text { days after } \\
\text { discharge. }\end{array}$ & $\begin{array}{l}\text { Measurement Tools: } \\
\text { Hand grip strength (in } \\
\text { kg) was measured with } \\
\text { a Jamar 5030J1 } \\
\text { Hydraulic Hand } \\
\text { Dynanometer. } \\
\text { Mobility was assessed } \\
\text { using the validated de } \\
\text { Morton Mobility Index } \\
\text { (DEMMI). } \\
\text { Restoration of function } \\
\text { after discharge was } \\
\text { assessed by means of } \\
\text { the Functional } \\
\text { Recovery } \\
\text { Score (FRS). } \\
\text { Reliability/validity } \\
\text { reported? } \\
\text { 1. }\end{array}$ & $\begin{array}{l}\text { Number of } \\
\text { Components: } 2 \\
\text { Type: } 3 \\
\text { (educational and } \\
\text { supportive care) } \\
\text { Mode } \\
\text { Medium: } 4 \\
\text { (face-to-face and } \\
\text { telephone follow-up). } \\
\text { Format: } 1 \text {. } \\
\text { Treatment Provider: } \\
3 \text { (Registered Dietitian } \\
\& \\
\text { General Practitioner) } \\
\text { Dose } \\
\text { Amount: } 120 \\
\text { minutes. } \\
\text { Frequency: one, } \\
\text { three, and eight weeks } \\
\text { after discharge. } \\
\text { Duration: } 8 \text { weeks. } \\
\text { Heterogeneity: } 1 ; 3 \\
\text { Approach: } 3 \text {. }\end{array}$ & $\begin{array}{l}\text { Indicator of } \\
\text { quality used: } \\
2 . \\
\text { Study } \\
\text { Findings/Statistica } \\
\text { I Significance: } \\
\text { Outcome \#1:1 } \\
\text { Intervention: } 00 \text {. } \\
\text { Control: } 00 \text {. }\end{array}$ \\
\hline $\begin{array}{l}\text { Study ID: } 2 \\
\text { Review Author ID: } 1 \\
\text { Citation: } \\
\text { Aguado, O., MD, Morcillo, } \\
\text { Cèsar, MD, PhD, Delàs, } \\
\text { Jordi, MD, PhD, Rennie, M., } \\
\text { MD, Bechich, S., MD, } \\
\text { Schembari, A., PhD, .. . } \\
\text { Rosell, Franscesc, MD, PhD. } \\
\text { (2010). Long-term } \\
\text { implications of a single home- } \\
\text { based educational } \\
\text { intervention in patients with } \\
\text { failure. Heart \& Lung: The } \\
\text { Journal of Acute and Critical } \\
\text { Care, 39(6), S14-S22. } \\
\text { doi:10.1016/j.hrtlng.2010.04. } \\
\text { 010 } \\
\text { Date of Study: } 2010\end{array}$ & $\begin{array}{l}\text { Purpose: The objective } \\
\text { of the study was to } \\
\text { evaluate the } \\
\text { effectiveness of a single } \\
\text { home-based educational } \\
\text { intervention for patients } \\
\text { admitted with heart } \\
\text { failure. } \\
\text { Study Design: } 3 \text {. } \\
\text { Quality Rating: } 1 \text {. } \\
\text { Global Rating (for } \\
\text { weak/moderate } \\
\text { articles): } 1 ; 6 \text {. } \\
\text { N=106 } \\
\text { Setting: } 5 \text {. } \\
\text { Country: } 3 \text {. } \\
\text { Medical Diagnosis: } 1 \text {. } \\
\text { Age: } 65+\end{array}$ & $\begin{array}{l}\text { Total number of } \\
\text { intervention groups: } 2 \\
\text { Number of participants } \\
\text { allocated to each } \\
\text { intervention group: } \\
\text { Control: } 64 \\
\text { Intervention: } 42 \\
\text { Outcomes and time } \\
\text { points collected and } \\
\text { reported: } \\
\text { Readmission rates (1); } \\
\text { mortality (1); emergency } \\
\text { department visits (9). } \\
\text { Assessed by MD on } 2 \\
\text { occasions; at } 6 \text { months } \\
\text { and } 24 \text { months. }\end{array}$ & $\begin{array}{l}\text { Measurement Tools: } \\
\text { Minnesota Living with } \\
\text { Heart Failure } \\
\text { Questionnaire } \\
\text { (MLWHFQ). } \\
\text { The Short Form-36 } \\
\text { (SF-36) } \\
\text { Reliability/validity } \\
\text { reported? } \\
\text { 3. }\end{array}$ & $\begin{array}{l}\text { Number of } \\
\text { Components: } 3 \\
\text { Type: } 3 \text { (education, } \\
\text { supportive care, } \\
\text { medication } \\
\text { management). } \\
\text { Mode } \\
\text { Medium: } 1 . \\
\text { Format: } 1 . \\
\text { Treatment Provider: } \\
1 \\
\text { Dose } \\
\text { Amount: } 120 \text { mins. } \\
\text { Frequency: one week } \\
\text { after discharge. } \\
\text { Duration: once. } \\
\text { Heterogeneity: } 1 ; 4 . \\
\text { Approach: } 1 .\end{array}$ & $\begin{array}{l}\text { Indicator of } \\
\text { Quality Used: } 3 . \\
\text { Study } \\
\text { Findings/Statistica } \\
\text { I Significance: } \\
\text { Outcome \#1: } 1 \\
\text { Statistical } \\
\text { Significance: } \\
\text { Intervention: } 1 \\
\text { (P=.003) } \\
\text { Control: } 00 . \\
\text { Outcome \#2: } 1 \\
\text { Statistical } \\
\text { Significance: } \\
\text { Intervention: } 1 . \\
\text { (P=.001). } \\
\text { Control: } 00 . \\
\\
\text { Outcome \#3: } 2 \\
\text { Statistical } \\
\text { Significance: } \\
\text { Intervention: } 00 . \\
\text { Control: } 00 \text {. }\end{array}$ \\
\hline $\begin{array}{l}\text { Study ID: } 3 \text {; Study found } \\
\text { within the Le Berre text. } \\
\text { Review Author ID: } 1 \text {. }\end{array}$ & $\begin{array}{l}\text { Purpose: } \\
\text { To evaluate the } \\
\text { effectiveness and cost- } \\
\text { effectiveness of a } \\
\text { community nurse- }\end{array}$ & $\begin{array}{l}\text { Total number of } \\
\text { intervention groups: } \\
\text { Two. } \\
\text { Number of participants } \\
\text { allocated to each }\end{array}$ & $\begin{array}{l}\text { Measurement Tools: } \\
\text { Abbreviated Mental } \\
\text { Test (AMT) } \\
\text { General Health } \\
\text { Questionnaire (GHQ) }\end{array}$ & $\begin{array}{l}\text { Component Number: } \\
\text { Three. } \\
\text { Type: } 3 \text { (supportive } \\
\text { care, organizational, }\end{array}$ & $\begin{array}{l}\text { Indicator of } \\
\text { quality used: } 2 \text {. } \\
\text { Study Findings: } \\
\text { Study outcome \#1: }\end{array}$ \\
\hline
\end{tabular}




\section{Citation:}

Kwok, T., Lee, J., Woo, J., Lee, D. T., \& Griffith, S. (2008). A randomized controlled trial of a community nurse-supported hospital discharge programme in older patients with chronic heart failure. Journal of Clinical Nursing, 17(1), 109117. doi:10.1111/j.13652702.2007.01978.x

Date of Study: 2008

Study ID: 4; Study found within the Kirk text.

\section{Review Author ID: 1.}

\section{Citation:}

Courtney, M., Edwards, H., Chang, A., Parker, A. Finlayson, K., \& Hamilton, K. (2009). Fewer emergency readmissions and better quality of life for older adults at risk of hospital readmission: A randomized controlled trial to determine the effectiveness of a 24-week exercise and telephone follow-up program. Journal of the American Geriatrics Society, 57(3), 395. doi:10.1111/j.15325415.2009.02138.x

Date of Study: 2009.

\section{Study ID: 5; CINAHL}

Review Author ID: 01

\section{Citation:}

Ebrahimi, Z., Eklund, K., Dahlin-Ivanoff, S., Jakobsson, A., \& Wilhelmson, K. (2017). Effects of a continuum of care intervention on frail elders' self-rated health, experiences of security/safety and symptoms: A randomised supported hospital

discharge program in preventing hospital re-admissions, improving functional status and handicap of older patients with chronic heart failure.

Study Design: 3 . Quality Rating: 3. $\mathbf{N}=105$.

Setting: 1 .

Country: 2.

Medical Diagnosis; 1. Age: $60+$

(however, sample was all above 65)

\section{Purpose:}

To determine the effect of an intervention targeting patients at high risk of hospital readmission on emergency health service utilization, health-related quality of life, general health, psychosocial outcomes, functional ability and costeffectiveness and to compare the effect of the intervention with that of usual care.

Study Design: 3 . Quality Rating: 3. $\mathbf{N}=128$.

Setting: 1 .

Country: 4 .

Medical Diagnosis; 0. Age: 65+

Purpose: To evaluate the effects of the CC intervention on selfrated health, experiences of security/safety and symptoms.

Study Design: 3 . Quality Rating: 1. Global Rating (for weak/moderate articles): 1;4.

\section{intervention group: \\ Intervention: 49. \\ Control: 56. \\ Outcomes and time points collected and reported: \\ Readmission rates 1 . 6-month follow up.}

Total number of intervention groups:

Two.

Number of participants allocated to each intervention group: Intervention: 64. Control: 64 .

Outcomes and time points collected and reported: Emergency health service utilization (emergency hospital readmissions and visits to emergency department, general practitioner (GP), or allied health professional) (1) and health-related quality of life (3)

Data collected at baseline and 4, 12, and 24 weeks after discharge.

Total number of intervention groups: 2 number of participants allocated to each intervention group: Intervention $=85$ Control $=76$

outcomes and time points collected and reported:

Self-rated health (3) Experiences of
London Handicap
Scale (LHS).

Reliability/validity reported? 1 .
Measurement Tools:

Medical Outcomes Study 12-item Short

Form Survey (SF$12 \mathrm{v} 2 \mathrm{t}$ )

Berg Balance Scale

Timed Up and Go Test 6-minute walk test.

Reliability/validity reported? 1 .
Measurement Tools:

Short-Form Health

Survey (SF-36)

Goteborg quality of

life Instrument

Reports of validity/reliability? 1 .
Component Number: 2

Type: 3

(Organizational/

Supportive care)

Mode:

Medium: 4; face-toface/telephone

Format: 2 .

Treatment Provider: 3 ; nursing (case
Statistical significance:

Intervention: 00.

Control: 00 .

Indicator of quality used: 3 .

Study Findings:

Outcome \#1: 1

Statistical

Significance:

Intervention: 1

$(\mathrm{p}=0.007)$.

Control: 00

Outcome \#2: 1

Statistical

Significance:

Intervention: 1

$(\mathrm{p}=0.001)$.

Control: 00.

Outcome \#3: 3

Statistical

Significance:

Intervention: 1

$(\mathrm{p}=<0.001)$.

Control: 00 .

Indicator of quality used: 1 .

Study Findings:

Outcome \#1: 3

Statistical

Significance:

Intervention: 00 .

Control: 00.

Outcome\#2: 3

Statistical

Significance: 


\begin{tabular}{|c|c|c|c|c|c|}
\hline $\begin{array}{l}\text { controlled trial. Nordic } \\
\text { Journal of Nursing Research, } \\
\text { 37(1), 33-43. https://doi- } \\
\text { org.ezproxy.lib.ryerson.ca/10. } \\
\text { 1177/2057158516668710 } \\
\text { Date of Study: } 2017\end{array}$ & $\begin{array}{l}\mathbf{N}=161 \\
\text { Setting: } 4 . \\
\text { Country: } 3 \text {. } \\
\text { Medical Diagnosis: } 0 . \\
\text { Age: } 65+\end{array}$ & $\begin{array}{l}\text { security/safety baseline } \\
\text { (3) } \\
\text { Symptoms: based on } \\
\text { quality of life } \\
\text { instrument. (3) } \\
\text { Baseline at } 1-3 \text { weeks } \\
\text { post-discharge, and } \\
\text { again at } 3,6,12 \text { months. } \\
\text { outcome definition: } \\
\text { Self-rated health: 'In } \\
\text { general, you would say } \\
\text { your health is...' } \\
\text { followed by } \\
\text { responses on a five-point } \\
\text { Likert-type scale: } \\
\text { excellent, very } \\
\text { good, good, fair or poor. } \\
\text { Experiences of } \\
\text { security/safety: A single } \\
\text { question was asked to } \\
\text { evaluate. }\end{array}$ & & $\begin{array}{l}\text { manager), OT, PT, } \\
\text { SW } \\
\text { Dose: } \\
\text { Amount: Not reported } \\
\text { Frequency: } \\
\text { once/month (more } \\
\text { frequent if needed). } \\
\text { Duration: one year. } \\
\text { Heterogeneity: } 1 ; 3 \text {. } \\
\text { Approach: } 3 \text {. }\end{array}$ & $\begin{array}{l}\text { Intervention: } 00 . \\
\text { Control: } 00 . \\
\text { Outcome\#3: } 3 \\
\text { Statistical } \\
\text { Significance: } \\
\text { Intervention: } 00 . \\
\text { Control: } 00 .\end{array}$ \\
\hline $\begin{array}{l}\text { Study ID: 6; CINAHL } \\
\text { Review Author ID: } 1 . \\
\text { Citation: } \\
\text { Berglund, H., Hasson, H., } \\
\text { Kjellgren, K., \& Wilhelmson, } \\
\text { K. (2015). Effects of a } \\
\text { continuum of care } \\
\text { intervention on frail older } \\
\text { persons' life satisfaction: a } \\
\text { randomized controlled study. } \\
\text { Journal of Clinical Nursing, } \\
\text { 24(7/8), 1079-1090. } \\
\text { https://doi- } \\
\text { org.ezproxy.lib.ryerson.ca/10. } \\
\text { 1111/jocn.12699 } \\
\text { Date of Study: } \\
\text { 2015 }\end{array}$ & $\begin{array}{l}\text { Purpose: } \\
\text { The aim of the project } \\
\text { was to } \\
\text { increase quality of care } \\
\text { and maintain functional } \\
\text { ability } \\
\text { among frail older } \\
\text { persons as well as to } \\
\text { increase cost } \\
\text { effectiveness. } \\
\text { Study Design: } \\
3 \text {. } \\
\text { Quality Rating: } 1 \text {. } \\
\text { Global Rating (for } \\
\text { weak/moderate } \\
\text { articles): } 1 ; 4 \text {. } \\
\text { N=161 } \\
\text { Setting: } 4 \text {. } \\
\text { Country: } 3 \text {. } \\
\text { Medical Diagnosis; } 0 \text {. } \\
\text { Age: } 65-79 \text {. }\end{array}$ & $\begin{array}{l}\text { Total number of } \\
\text { intervention groups: } 2 \\
\text { number of participants } \\
\text { allocated to each } \\
\text { intervention group: } \\
\text { Intervention } n=85, \\
\text { control } n=76 \\
\text { Outcomes and time } \\
\text { points collected and } \\
\text { reported: } \\
\text { life satisfaction (3) } \\
\text { Baseline measurement } \\
\text { was usually made within } \\
\text { a few days } \\
\text { after hospital discharge, } \\
\text { and follow-ups were } \\
\text { performed at } \\
\text { three, six and } 12 \text { months } \\
\text { after the baseline } \\
\text { measurement }\end{array}$ & $\begin{array}{l}\text { Measurement Tools: } \\
\text { LiSat-11 scale } \\
\text { was used to measure } \\
\text { older persons' life } \\
\text { satisfaction. } \\
\text { Reports of } \\
\text { validity/reliability: } 1 .\end{array}$ & $\begin{array}{l}\text { Component Number: } \\
2 \\
\text { Type: } 3 ; \\
\text { (Organizational } \\
\text { Supportive care) } \\
\text { Mode } \\
\text { Medium: } 4 \text { (face-to- } \\
\text { face/telephone) } \\
\text { Format: } 2 \text {. } \\
\text { Treatment Provider: } \\
3 \text { nursing (case } \\
\text { manager), OT, PT, } \\
\text { SW } \\
\text { Dose } \\
\text { Amount: Not reported } \\
\text { Frequency: } \\
\text { once/month (more } \\
\text { frequent if needed). } \\
\text { Duration: one year. } \\
\text { Heterogeneity: } 1 ; 3 \text {. } \\
\text { Approach: } 3 \text {. }\end{array}$ & $\begin{array}{l}\text { Indicator of } \\
\text { quality used: } \\
1 . \\
\text { Study Findings: } \\
\text { Outcome \#1: } 3 \\
\text { Statistical } \\
\text { Significance: } \\
\text { Three-month } \\
\text { follow-up: } \\
\text { Intervention: 00. } \\
\text { Control: 00. } \\
\text { 6-month follow-up: } \\
\text { Intervention group: } \\
\text { 00. } \\
\text { Control: } 1 . \\
\text { Psychological } \\
\text { health } \\
\text { 12-month follow- } \\
\text { up: } \\
\text { Intervention group: } \\
\text { 1. } \\
\text { Financial situation } \\
\text { (p=0. 04) } \\
\text { Functional capacity } \\
\text { (p=0.01) } \\
\text { Psychological } \\
\text { health (p=0.00). } \\
\text { Control group: 00. }\end{array}$ \\
\hline $\begin{array}{l}\text { Study ID: 7; CINAHL } \\
\text { Review Author ID: } 1 . \\
\text { Citation: } \\
\text { Altfeld, S. J., Shier, G. E., } \\
\text { Rooney, M., Johnson, T. J., } \\
\text { Golden, R. L., Karavolos, K., }\end{array}$ & $\begin{array}{l}\text { Purpose: } \\
\text { To identify needs } \\
\text { encountered by older } \\
\text { adult patients after } \\
\text { hospital } \\
\text { discharge and assess the } \\
\text { impact of a telephone } \\
\text { transitional }\end{array}$ & $\begin{array}{l}\text { Total number of } \\
\text { intervention groups: } \\
\text { Two } \\
\text { number of participants } \\
\text { allocated to each } \\
\text { intervention group: } \\
360 \text { intervention } \\
360 \text { control }\end{array}$ & None. & $\begin{array}{l}\text { Component Number: } \\
\text { one } \\
\text { Type: } 2 \text {; (Supportive } \\
\text { care) } \\
\text { Mode } \\
\text { Medium: } 2 \text {. } \\
\text { Format: } 2 \text {. }\end{array}$ & $\begin{array}{l}\text { Indicator of } \\
\text { quality used: } 3 \text {. } \\
\text { Study Findings: } \\
\text { Outcome \# 1: } 1 \\
\text { Statistical } \\
\text { significance: }\end{array}$ \\
\hline
\end{tabular}




\begin{abstract}
... Perry, A. J. (2013). Effects of an Enhanced Discharge Planning Intervention for Hospitalized Older Adults: A Randomized Trial.

Gerontologist, 53(3), 430440. https://doiorg.ezproxy.lib.ryerson.ca/ger ont/gns109
\end{abstract}

\section{Date of Study:}

2013

\section{Study ID: 8; CINAHL}

Review Author ID: 1 .

\section{Citation:}

\section{Li Xueyu, Yu Hao, Xu} Shunlin, Li Rongbin, \& Gao Yuan. (2017). Effects of LowIntensity Exercise in Older Adults With Chronic Heart Failure During the Transitional Period From Hospital to Home in China: A Randomized Controlled Trial. Research in Gerontological Nursing, 10(3), 121-128. https://doiorg.ezproxy.lib.ryerson.ca/10. 3928/19404921-20170411-02

Date of Study: 2017.

\section{Study ID: 9}

\section{Review Author ID: 1}

\section{Citation:}

Parry C, Min S, Chugh A Chalmers $S$, \& Coleman EA. (2009). Further application of the care transitions care intervention on stress, health care utilization, readmissions, and mortality.

Study Design: 3 . Quality Rating: 1. Global Rating (for weak/moderate articles): 4;5. $\mathbf{N}=720$

Setting: 3 .

Country: 1 .

Medical Diagnosis; 0. Age: $65+$
Outcomes and time points collected and reported:

Patient and caregiver stress (3), physician follow-up (1), mortality (2).

Follow-up: 30-60-days post-discharge readmission (1), and

Treatment Provider:

4 (social worker).

Dose

Amount: Unclear Frequency: mean number of contacts $=$ $5.4(\mathrm{SD}=6.3)$.

Duration: 5.8

$(\mathrm{SD}=11.3)$ days on average.

Heterogeneity: 1;3.

Approach: 1

\section{Purpose:}

To investigate the effect of an exercise protocol initiated during hospitalization on

HRQoL,

physical function, and heart function in older adults ( $\geq 70$ years)

Study Design: 3 . Quality Rating: 3 . $\mathbf{N}=78$

Setting: 0 .

Country: 2. Medical Diagnosis: 1 . Age: $70+$

\section{Measurement Tools:}

Total number of intervention groups: Two

Number of participants allocated to each intervention group: Intervention group: $(\mathbf{n}=40)$

Control group $(\mathbf{n}=38)$.

Outcomes and time points collected and reported:

Health-related quality of life (3) physical function (2), and heart function (2)

Baseline and 12-weeks post-discharge.
HRQoL was measured using the Minnesota

Living with

Heart Failure

Questionnaire (MLHFQ).

reports of reliability/validity of instruments. 1 .
Total number of

Purpose:

to test whether a selfcare model for transitional care that has been demonstrated to improve outcomes in Medicare

\section{intervention groups: 2}

Number of participants allocated to each intervention group: 44- intervention 42- control
Component Number: one.

Type: 2 (supportive care).

Mode

Medium: 4; (face-to-

face, written materials, telephone)

Format: 3 .

Treatment

Providers: 3 (Nurses, physicians, pharmacists, and nutritionists).

$\underline{\text { Dose }}$

Amount: unclear.

Frequency:

$1 /$ week for first month post-discharge

Every 2 weeks during the second month

Once during the third month.

Duration: 12 weeks post-discharge.

Heterogeneity: 2;4.

Approach: 1

Component

Number:

Three.

Type: 3

(Educational, supportive care, medication management).
Intervention: 1 .

Physician

communication

( $\mathrm{p}=.002)$.

Physician apt

scheduled (<.001).

Physician apt kept

(<.001).

Control: 00 .

Outcome \#2: 3 :

Statistical

Significance:

Intervention: 00.

Control: 00 .

Outcome \#3: 1

Intervention: 00.

Control: 00 .

Outcome \#4: 2

Intervention: 00.

Control: 00.

Indicator of

quality used: 1 .

Study

Findings/statistical significance:

Outcome \#1: 3

Significant

improvement:

Intervention: 01.

$(\mathrm{p}=0.05)$.

Control: 00.

Outcome \#2: 2

Significant improvement:

Intervention: 1 .

6MWD,

and TUG scores ( $\mathrm{p}$

$<0.05$ )

Control: 00 .

Outcome \#3: 2

Statistical

significance:

Intervention: 00.

Control: 00.

\section{Indicator of} quality used: 2.

Study

Findings/Statistical Significance:

Outcome \#1: 1 


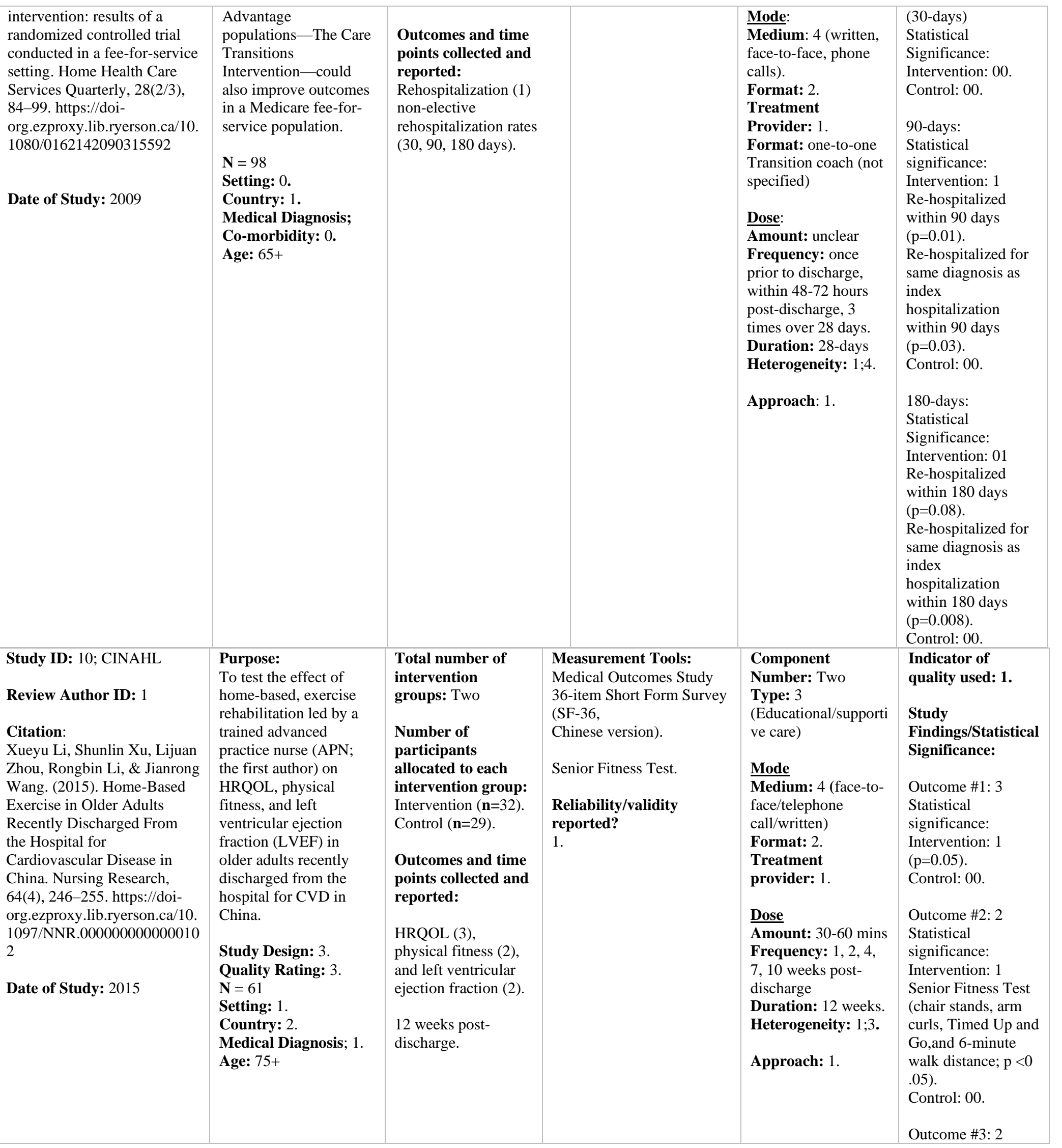




\begin{tabular}{|c|c|c|c|c|c|}
\hline & & & & & $\begin{array}{l}\text { Statistical } \\
\text { significance: } \\
\text { Intervention: } 00 . \\
\text { Control: } 00 .\end{array}$ \\
\hline $\begin{array}{l}\text { Study ID: 11; CINAHL } \\
\text { Review Author ID: } 1 \\
\text { Citation: } \\
\text { Ekelund, C., \& Eklund, K. } \\
\text { (2015). Longitudinal effects } \\
\text { on self-determination in the } \\
\text { RCT “Continuum of care for } \\
\text { frail elderly people.” Quality } \\
\text { in Ageing \& Older Adults, } \\
\text { 16(3), 165-176. https://doi- } \\
\text { org.ezproxy.lib.ryerson.ca/10. } \\
\text { 1108/QAOA-12-2014-0045 } \\
\text { Date of Study: } 2015\end{array}$ & $\begin{array}{l}\text { Purpose: } \\
\text { To evaluate the effect of } \\
\text { the "Continuum of care } \\
\text { for frail elderly people" } \\
\text { in terms of the } \\
\text { self-determination in } \\
\text { daily life of community- } \\
\text { dwelling frail older } \\
\text { persons. } \\
\text { Study Design: } 3 \text {. } \\
\text { Quality Rating: } 1 \text {. } \\
\text { Global Rating (for } \\
\text { weak/moderate } \\
\text { articles): } 1 ; 4 \\
\text { N = } 158 \text {. } \\
\text { Setting: } 5 \text {. } \\
\text { Country: } 3 \text {. } \\
\text { Medical Diagnosis; } 0 \text {. } \\
\text { Age: } 65+\end{array}$ & $\begin{array}{l}\text { Total number of } \\
\text { intervention } \\
\text { groups: } \\
\text { Two } \\
\text { number of } \\
\text { participants } \\
\text { allocated to each } \\
\text { intervention group: } \\
\text { Intervention group: } \\
85 \\
\text { Control: } 76 \\
\text { Outcomes and time } \\
\text { points collected and } \\
\text { reported: } \\
\text { Outcome: self- } \\
\text { determination (3) } \\
\text { three, six, twelve } \\
\text { months. }\end{array}$ & $\begin{array}{l}\text { Outcome Definition: } \\
\text { self-determination in: } \\
\text { mobility (four items), self- } \\
\text { care (five items), activities } \\
\text { in and around the house } \\
\text { (four items), financial } \\
\text { situation (one item), use of } \\
\text { time (one item), social } \\
\text { relationships (five items), } \\
\text { help and support others } \\
\text { (one item) and, a summary } \\
\text { (one item), a total of } 22 \\
\text { items. } \\
\text { Measurement Tool Used: } \\
\text { Impact on Participation and } \\
\text { Autonomy } \\
\text { for Older persons (IPA-O) } \\
\text { Reports of } \\
\text { validity/reliability: } 1 .\end{array}$ & $\begin{array}{l}\text { Component } \\
\text { Number: two } \\
\text { Type: } 3 \\
\text { (Organizational/supp } \\
\text { ortive care). } \\
\text { Mode } \\
\text { Medium: } 1 \text {. } \\
\text { Format: } 2 \text {. } \\
\text { Treatment } \\
\text { Providers: } 3 \\
\text { (professionals in } \\
\text { nursing, occupational } \\
\text { therapy, } \\
\text { physiotherapy and } \\
\text { social work). } \\
\text { Amount: Unclear } \\
\text { Frequency: within } \\
\text { one-week post- } \\
\text { discharge, then } \\
\text { once/month } \\
\text { Duration: minimum } \\
\text { of one-year. } \\
\text { Heterogeneity: } 1 ; 4\end{array}$ & $\begin{array}{l}\text { Indicator of } \\
\text { quality used: } \\
1 . \\
\text { Study Findings: } \\
\text { Outcome\# 1: } 3 \text {. } \\
\text { Statistical } \\
\text { significance: } \\
\text { (3-month follow- } \\
\text { up): } \\
\text { Intervention: } 01 \text {. } \\
\text { Activities around } \\
\text { the house } \\
\text { Social relationships } \\
\text { Control: } 00 \text {. } \\
\text { (6-month follow- } \\
\text { up): } \\
\text { Intervention: } 01 \\
\text { Social relationships } \\
\text { Control: } 00 \\
\text { (12-month follow- } \\
\text { up) } \\
\text { Intervention: } 00 \text {. } \\
\text { Control: } 00 \text {. }\end{array}$ \\
\hline & & & & Approach: 3 . & \\
\hline $\begin{array}{l}\text { Study ID: 12: CINAHL } \\
\text { Review Author ID: } 1 \\
\text { Citation: } \\
\text { Lindegaard Pedersen, J., } \\
\text { Pedersen, P., \& Damsgaard, } \\
\text { E. (2017). Nutritional follow- } \\
\text { up after discharge prevents } \\
\text { readmission to hospital - A } \\
\text { randomized clinical trial. } \\
\text { Journal of Nutrition, Health \& } \\
\text { Aging, 21(1), 75-82. } \\
\text { https://doi- } \\
\text { org.ezproxy.lib.ryerson.ca/10. } \\
\text { 1007/s12603-016-0745-7 } \\
\text { Date of Study: } 2017\end{array}$ & $\begin{array}{l}\text { Purpose: To compare } \\
\text { the effects of two } \\
\text { individualized } \\
\text { nutritional follow-up } \\
\text { intervention strategies } \\
\text { (home visit or telephone } \\
\text { consultation) with no } \\
\text { follow-up, with regard } \\
\text { to acute readmissions to } \\
\text { hospital at two points in } \\
\text { time, } 30 \text { and } 90 \text { days } \\
\text { after discharge from } \\
\text { hospital. } \\
\text { Study Design: } 3 \text {. } \\
\text { Quality Rating: } 1 . \\
\text { Global Rating (for } \\
\text { weak/moderate } \\
\text { articles): } 4 ; 6 \\
\text { N= } 208 \\
\text { Setting: } 4 \text {. } \\
\text { Country: } 3 \text {. } \\
\text { Medical Diagnosis; } 2 \text {. } \\
\text { Age: } 75+\end{array}$ & $\begin{array}{l}\text { Total number of } \\
\text { intervention } \\
\text { groups: } \\
\text { Three. } \\
\text { number of } \\
\text { participants } \\
\text { allocated to each } \\
\text { intervention group: } \\
\text { Intervention \#1 } \\
\text { (home visits): } 73 \\
\text { Intervention \#2 } \\
\text { (telephone } \\
\text { consultation): } 68 \\
\text { Control (no follow- } \\
\text { up): } 67 \\
\text { Outcomes and time } \\
\text { points collected and } \\
\text { reported: } \\
\text { Readmission rates } \\
\text { (1) } \\
\text { 30- and 90-days post } \\
\text { discharge }\end{array}$ & None. & $\begin{array}{l}\text { Intervention Group } \\
\text { \#1 (home visits) } \\
\text { Component } \\
\text { Number: Two. } \\
\text { Type: } 3 \text { (supportive } \\
\text { care/educational). } \\
\text { Mode } \\
\text { Medium: } 1 . \\
\text { Format: } 2 . \\
\text { Treatment } \\
\text { Provider: } 4 \text {; Clinical } \\
\text { dietitian } \\
\text { Dose } \\
\text { Amount: Home } \\
\text { visits: } 45 \text { mins } \\
\text { Frequency: one, } \\
\text { two, four weeks post } \\
\text { discharge. } \\
\text { Duration: } 30 \text { days. } \\
\text { Heterogeneity: } 1 ; 4 . \\
\text { Approach: } 2 . \\
\text { Intervention Group } \\
\text { \#2 (phone } \\
\text { consultation) } \\
\text { Component } \\
\text { Number: Two. }\end{array}$ & $\begin{array}{l}\text { Indicator of } \\
\text { quality used: } \\
3 . \\
\text { Study } \\
\text { Findings/statistical } \\
\text { significance: } \\
\text { Outcome \#1: } 1 \\
30 \text { days: } \\
\text { Intervention\#1: } 1 \\
\text { (p=0.03) } \\
\text { Intervention 2: } 00 \\
\text { Control: } 00 . \\
\text { 90 days: } \\
\text { Intervention \#1: } 1 \\
\text { (p<0.01) } \\
\text { Intervention \#2: } 00 \\
\text { Control: } 00\end{array}$ \\
\hline
\end{tabular}




\section{Study ID: 13; CINAHL}

Review Author ID: 1

\section{Citation:}

Clemson, L., Lannin, N. A., Wales, K., Salkeld, G., Rubenstein, L., Gitlin, L., ... Cameron, I. D. (2016). Occupational Therapy Predischarge Home Visits in Acute Hospital Care: A Randomized Trial. Journal of the American Geriatrics Society, 64(10), 2019-2026. https://doiorg.ezproxy.lib.ryerson.ca/10. 1111/jgs.14287

Date of Study: 2016.

\section{Purpose:}

To determine whether an enhanced occupational therapy discharge planning intervention that involved pre- and postdischarge home visits, goal setting, and followup (the HOME program) would be superior to a usual care intervention.

Study Design: 3 . Quality Rating: 2. Global Rating (for weak/moderate articles): 5 .

$\mathrm{N}=93$

Setting:

3.

Country: 4.

Medical Diagnosis; 0. Age: 70+

\section{Purpose:}

To evaluate if homebased rehabilitation of inpatients improved outcome compared to standard care.

Study Design: 3 . Quality Rating: 2. Global Rating (for weak/moderate articles): 4 . $\mathrm{N}=61$

Setting: 5 .

Country: 3 .
Total number of intervention groups: Two.

number of participants allocated to each intervention group: Intervention group: 47

Control:46.

Outcomes and time points collected and reported: Primary: ADLs, participation in life roles and activities (2). Follow Up: 90 days.

\section{Total number of} intervention groups: Two Number of participants allocated to each intervention group: Intervention: 38

Control: 33

Outcomes and time points collected and reported:

The primary outcome measure was the
Measurement Tools:

Nottingham Extended Activities of Daily Living scale (NEADL)

Life Disability Index (LLDI)

\section{Reports of} Validity/reliability? 1.
Type: 5 (supportive care/educational).

Mode

Medium: 2.

Format: 2 .

Treatment

Provider: 4; Clinical dietitian

Dose

Amount:

Telephone

consultation: 15

mins.

Frequency: one, two, four weeks post discharge.

Duration: 30 days.

Heterogeneity: 1;4.

Approach: 2.

Component

Number: Two

Type: 3; (Supportive care/organizational)

\section{Mode}

Medium: 4

(telephone, face-to-

face).

Format: 2 .

Treatment

Provider: 4 .

Dose

Amount: Not

described.

Frequency: a pre-

discharge home visit, a post-discharge visit within the first week after discharge, and telephone calls at 2 and 4 weeks

Duration: one month.

Heterogeneity: 1;4.

Approach: 2.

Component

Number: 2.

Type: 3 (Supportive care/educational)

$\underline{\text { Mode }}$

Medium: 4 (face-toface and written materials).

Format: 1 .

Treatment

Provider: 3 (nurse, physiotherapists, occupational therapists and
Indicator of quality used: 1 .

Study

Findings/statistical significance:

Outcome \#1: 2

Statistical significance: Intervention: 00. Control: 00.

Indicator of quality used: 1.

Study Findings:

Outcome \#1: 2

Statistical significance:

Intervention: 01 .

$(\mathrm{p}=0.04)$.

Control: 00. 


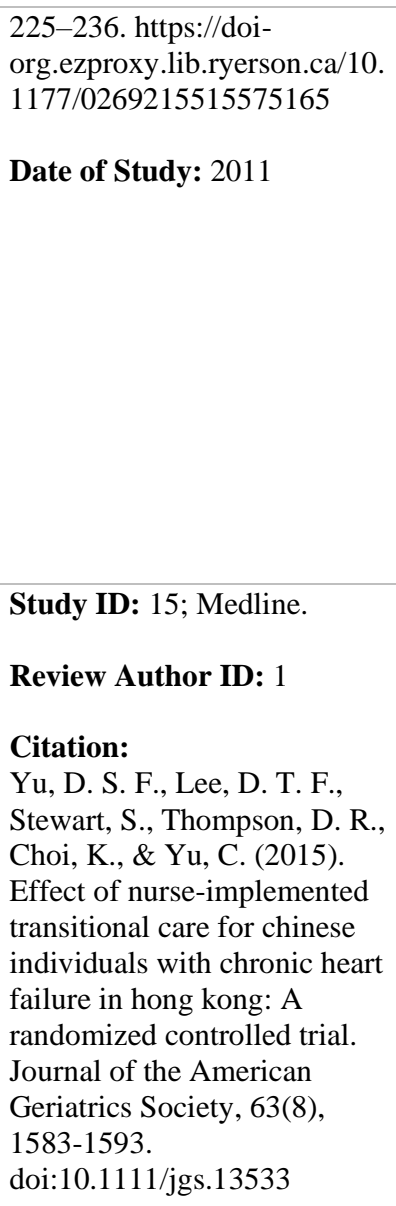

Date of Study: 2015

\section{Medical Diagnosis: 3 .}

Age: 65+

A

\section{Purpose:}

To examine the effect of a nurse implemented TC model to support the post- discharge care of Chinese individuals with CHF in Hong Kong.

\section{Study Design: 3 .} Quality Rating: 2. Global Rating (for weak/moderate articles): 4 .

$\mathrm{N}=178$

Setting: 0 .

Country: 2. Medical Diagnosis; 1. Age: 60+ (however sample was all above 70 years of age).

Study ID: 16; Medline.

\section{Review Author ID: 1.}

\section{Citation:}

Thygesen, L. C., Fokdal, S., Gjørup, T., Taylor, R. S., Zwisler, A., Prevention of Early Readmission Research Group, \& on behalf of the Prevention of Early

\section{Purpose:}

To evaluate how municipality-based post-discharge followup visits including a general practitioner and municipal nurse affect early readmission among high-risk older people discharged from a hospital department of modified

Rankin Scale score

90 days after stroke

(2).

At baseline and after

90 days.

(not

\begin{tabular}{l|l|l|l}
\hline Total number of & Measurement Tools: & $\begin{array}{l}\text { Component } \\
\text { Number: Two. }\end{array}$ & $\begin{array}{l}\text { Indicator of } \\
\text { quality used: } 3 .\end{array}$ \\
\hline Thtervention & The 18-item Chinese & Type: 3 &
\end{tabular}

groups: Two

Number of

participants

allocated to each

intervention group:

Control group:

$(\mathbf{n}=88)$.

Intervention Group:

$(\mathbf{n}=90)$.

Outcomes and time

points collected and

reported:

event-free

Survival (2), all-

cause hospital

readmission (1), and

mortality (2) during

the 9-month follow-

up.

6 weeks (T1), 3

months (T2), and 9

months (T3) after

hospital discharge.

version of the Self-Care

Heart Failure

Index (SCHFI), version

Type: 3

(Education/supportiv

e care).

Dutch Heart Failure

Knowledge Scale (DHFKS)

Mode

Medium: 4 (face-to-

face, telephone call).

Format: 1 .

Treatment

Providers: 1 .

version of the Minnesota

Living with Heart Failure

Questionnaire (MLHFQ)

The Chinese version of the EuroQoL 5-Dimensional Questionnaire (EQ-5D) measures generic HRQL.

\section{Reliability/Validity} reported? 1 .

\section{Dose}

Amount: Not

described.

Frequency: pre-

discharge visit, two

home visits, phone

calls ( 1 week after

the second home

visit, then every 2

weeks for three

months, then every 2

months for 6 months)

(over 9 months). (for

a total of nine calls

during the discharge

period).

Duration: 9-

months.

Heterogeneity: 2;3.

Approach: 3 .

\begin{tabular}{|l|l|} 
Total number of & None. \\
intervention & \\
groups: Two & \\
Number of & \\
participants & \\
allocated to each & \\
intervention group: & \\
Intervention: 270 & \\
Control: 261 & \\
\hline Outcomes and time & \\
\hline
\end{tabular}

\section{Component}

Number: Two

Type: 3 (supportive care/medication management)

Mode

Medium: 1.

Format: 1 .

Treatment

Provider: 3.
Study Findings:

Outcome \#1: 2

Statistical

Significance:

Intervention: 1

$(\mathrm{p}=0.03$ at 9

months).

Control: 00.

Outcome \#2: 1

Statistical

Significance:

Intervention group:

$1(\mathrm{P}=.048$ during

first 6 weeks).

Control: 00.

Outcome \#3: 2

Statistical

significance:

Intervention: 00.

Control: 00.

Indicator of quality used: 2.

Study

Findings/Statistical Significance:

Outcome \#1: 1

Statistical

significance: 


\begin{tabular}{|c|c|c|c|c|c|}
\hline $\begin{array}{l}\text { Readmission Research Group. } \\
\text { (2015). Can municipality- } \\
\text { based post-discharge follow- } \\
\text { up visits including a general } \\
\text { practitioner reduce early } \\
\text { readmission among the fragile } \\
\text { elderly (65+ years old)? A } \\
\text { randomized controlled trial. } \\
\text { Scandinavian Journal of } \\
\text { Primary Health Care, } 33(2) \text {, } \\
\text { 65-73. } \\
\text { doi:10.3109/02813432.2015.1 } \\
041831\end{array}$ & $\begin{array}{l}\text { internal medicine. } \\
\text { Study Design: } 1 \\
\text { Quality Rating: } 3 \text {. } \\
\mathrm{N}=531 \\
\text { Setting: } 1 \\
\text { Country: } 3 \\
\text { Medical Diagnosis: } 0 \\
\text { Age: } 65+\end{array}$ & $\begin{array}{l}\text { points collected and } \\
\text { reported: } \\
\text { readmission }(1) \\
\text { within } 30 / 180 \text { days. }\end{array}$ & & $\begin{array}{l}\text { Dose } \\
\text { Amount: } 60 \\
\text { minutes. } \\
\text { Frequency: within } 7 \\
\text { days of discharge, } \\
\text { 3rd week, and 8th } \\
\text { week after discharge. } \\
\text { Duration: } 8 \text { weeks. } \\
\text { Heterogeneity: } 1 ; 4 . \\
\text { Approach: } 1 .\end{array}$ & $\begin{array}{l}\text { Intervention: } 00 . \\
\text { Control: } 00 .\end{array}$ \\
\hline $\begin{array}{l}\text { Date of Study: } 2015 . \\
\text { Study ID: 17; Medline } \\
\text { Review Author ID: } 1 . \\
\text { Citation: } \\
\text { Beck, A., Andersen, U., } \\
\text { Leedo, E., Jensen, L., } \\
\text { Martins, K., Quvang, M., .. } \\
\text { Rønholt, F. (2015). Does } \\
\text { adding a dietician to the } \\
\text { liaison team after discharge of } \\
\text { geriatric patients improve } \\
\text { nutritional outcome: A } \\
\text { randomised controlled trial. } \\
\text { Clinical Rehabilitation, } \\
\text { 29(11), 1117-1128. } \\
\text { doi:10.1177/02692155145647 } \\
\text { 00 } \\
\text { Date of Study: } 2015\end{array}$ & $\begin{array}{l}\text { Purpose: } \\
\text { To test whether adding } \\
\text { a dietician to a } \\
\text { discharge Liaison-Team } \\
\text { after } \\
\text { discharge of geriatric } \\
\text { patients improves } \\
\text { nutritional status, } \\
\text { muscle strength and } \\
\text { patient relevant } \\
\text { outcomes } \\
\text { Study Design: } 3 \text {. } \\
\text { Quality Rating: } 3 \text {. } \\
\text { N=71 } \\
\text { Setting: } 5 \text {. } \\
\text { Country: } 3 \text {. } \\
\text { Medical Diagnosis: } 2 \text {. } \\
\text { Age: } 70+\text {. }\end{array}$ & $\begin{array}{l}\text { Total number of } \\
\text { intervention } \\
\text { groups: Two } \\
\text { Number of } \\
\text { participants } \\
\text { allocated to each } \\
\text { intervention group: } \\
\text { Intervention: } 34 \\
\text { Control: } 63 \\
\text { Outcomes and time } \\
\text { points collected and } \\
\text { reported: } \\
\text { Muscle strength } \\
\text { (hand grip strength) } \\
\text { (2) } \\
\text { Outcome parameters } \\
\text { were measured just } \\
\text { before } \\
\text { discharge and after } \\
12 \text { weeks in the } \\
\text { home of the } \\
\text { participants. } \\
\text { Evaluation of re- } \\
\text { /hospitalizations was } \\
\text { done after } 30 \text { days, } \\
12 \text { weeks and } 6 \\
\text { months after } \\
\text { discharge. And } \\
\text { evaluation of } \\
\text { mortality was done } \\
\text { after } 12 \text { weeks and } 6 \\
\text { months after } \\
\text { discharge. }\end{array}$ & $\begin{array}{l}\text { Measurement Tools: } \\
\text { Quality of Life was } \\
\text { assessed by means of } \\
\text { EuroQol5D-3L (EQ-5D- } \\
\text { 3L). } \\
\text { deMorton Mobility Index } \\
\text { (DEMMI) } \\
\text { Jamar 5030J1 Hydraulic } \\
\text { Hand Dynamometer. } \\
\text { Reliability/validity } \\
\text { reported? } 1 .\end{array}$ & $\begin{array}{l}\text { Component } \\
\text { Number: Two } \\
\text { Type: } 3 \text { (supportive } \\
\text { care, education). } \\
\text { Mode } \\
\text { Medium: } 4 \text { (face-to- } \\
\text { face/telephone } \\
\text { contact). } \\
\text { Format: } 1 . \\
\text { Treatment } \\
\text { Provider: } 4 \text {. } \\
\text { Dose } \\
\text { Amount: Not } \\
\text { described. } \\
\text { Frequency: Three } \\
\text { home visits. Day of } \\
\text { discharge, three } \\
\text { weeks, eight weeks } \\
\text { post-discharge. } * * \\
\text { telephone contact } \\
\text { between visits if } \\
\text { needed. } \\
\text { Duration: } 12-\text {-weeks. } \\
\text { Heterogeneity: } 1 ; 3 \text {. } \\
\text { Approach: } 3 \text {. }\end{array}$ & $\begin{array}{l}\text { Indicator of } \\
\text { quality used: } \\
3 . \\
\text { Study Findings: } \\
\text { Outcome \# 1: } 2 \\
\text { Statistical } \\
\text { Significance: } \\
\text { Intervention: } 00 . \\
\text { Control: } 00 .\end{array}$ \\
\hline $\begin{array}{l}\text { Study ID: 18; Medline } \\
\text { Review Author ID: } 1 \\
\text { Citation: } \\
\text { Berglund, H., Wilhelmson, } \\
\text { K., Blomberg, S., Dunér, A., } \\
\text { Hasson, H., Kjellgren, K. I., . }\end{array}$ & $\begin{array}{l}\text { Purpose: } \\
\text { To analyze frail older } \\
\text { people's views of } \\
\text { quality of care when } \\
\text { receiving a } \\
\text { comprehensive } \\
\text { continuum of care } \\
\text { intervention compared }\end{array}$ & $\begin{array}{l}\text { Total number of } \\
\text { intervention } \\
\text { groups: Two. } \\
\text { number of } \\
\text { participants } \\
\text { allocated to each } \\
\text { intervention group: } \\
\text { intervention } n=85\end{array}$ & $\begin{array}{l}\text { Measurement Tools: } \\
\text { the Pyramid Questionnaire } \\
\text { Reliability/Validity } \\
\text { reported? } \\
1 .\end{array}$ & $\begin{array}{l}\text { Component } \\
\text { Number: } 2 . \\
\text { Type: } 3 \\
\text { (Organizational/supp } \\
\text { ortive care) } \\
\text { Mode } \\
\text { Medium: } 4 \text { (face-to- }\end{array}$ & $\begin{array}{l}\text { Indicator of } \\
\text { quality used: } 1 . \\
\text { Study } \\
\text { Findings/statistical } \\
\text { significance: } \\
\text { Outcome \#1: } 3\end{array}$ \\
\hline
\end{tabular}


. Institute of Medicine, Department of Public Health and Community Medicine. (2013). Older people's views of quality of care: A randomised controlled study of continuum of care. Journal of Clinical Nursing, 22(1920)

Date of Study: 2013 with those of people receiving the usual care.

Study Design: 3 . Quality Rating: 1. Global Rating (for weak/moderate articles): $1 ; 4$ $\mathrm{N}=161$

Setting: 0 .

Country: 3 .

Medical Diagnosis; 0. Age: 65+. control $n=76$ )

Outcomes and time points collected and reported:

Older people's views of quality of care (3) were measured at three, six and 12 months after the baseline measurement, which was performed a few days after hospital discharge.

\section{Study ID: 19; Medline}

\section{Review Author ID: 1}

\section{Citation:}

Wong, F. K. Y., Chow, S. K. Y., Chan, T. M. F., \& Tam, S. K. F. (2014). Comparison of effects between home visits with telephone calls and telephone calls only for transitional discharge support: A randomised controlled trial. Age and Ageing, 43(1), 9197. doi:10.1093/ageing/aft123

Date of Study: 2014

\section{Purpose:}

To examine the overall effects of a transitional care program for discharged medical patients and the differential effects of telephone calls only.

Study Design: 3 . Quality Rating: 2. Global Rating (for weak/moderate articles): 4

$\mathbf{N}=610$

Setting: 1 .

Country: 2 .

Medical Diagnosis: 0 . Age: 65+

face/telephone

contact).

Format: 2 .

Treatment

Provider: 3 .

Dose

Amount: Not

described.

Frequency: Once in $\mathrm{ED}$, once on hospital ward, a few days after discharge/oneweek post-home visit.

Once a month on average.

Duration: 12-month average.

Heterogeneity: 1;3.

Approach: 2.

Total number of intervention groups: Three. number of participants allocated to each intervention group: Control: 210 (Intervention group 1) Home visits + Calls: 196

(Intervention group

2) Calls: 204

Outcomes and time points collected and reported:

Data were collected at the time of discharge (O1), at 4 weeks after discharge when the intervention program was completed $(\mathrm{O} 2)$ and at 12 weeks (O3).

The readmission data (1) were captured at 28 days (4 weeks) and 84 days (12 weeks) postdischarge.
Measurement Tools:

Quality of Life: MOS 36item Short Form Health Survey (SF-36)

Self-efficacy was measured by the short version Chronic Disease SelfEfficacy Scale

\section{Reliability/validity} reported? 1 .

\section{Intervention Group \\ \#1: Home visit} group:

Component

Number: Two.

Type: 3

(Organizational/supp

ortive care).

Mode

Medium: 4 (face-toface/telephone calls)

Format: 1 .

Treatment

Providers: 1.

\section{Dose:}

Amount: Not

described.

Frequency: predischarge, once/week 2 home visits (week 1 and 3 )

2 phone calls (weeks 2 and 4).

Duration: 4-weeks. Heterogeneity: 1;4.

\section{Approach: 1.}

Intervention Group \#2: Call group:

Component

Number: Two.

Type: 3

(Organizational/supp ortive care). 2
Statistical

significance:

(3-month follow-up)

Intervention: 1

Care planning $(<\mathrm{p}=$ 0.005 )

knowledge

of whom to contact

about care/service

( $\mathrm{p}<0.011)$.

Control: 00.

(6-Month followup)

Intervention: 00.

Control: 00.

(12-month followup)

Intervention: 1

Knowledge

of whom to contact about care/service ( $\mathrm{p}<0.027$ ).

Control: 00 .

Indicator of

quality used: 2 .

Study Findings:

Outcome \#1: 1

Statistical

significance:

4- weeks:

Intervention \#1: 1

$(\mathrm{P}=0.041)$

Intervention \#2: 00.

Control: 00 .

Outcome \#1: 1

12- weeks:

Statistical

Significance:

Intervention \#1: 00.

Intervention \#2: 00.

Control: 00 . 


\begin{tabular}{|c|c|c|c|c|c|}
\hline & & & & $\begin{array}{l}\text { Mode } \\
\text { Medium: } 2 . \\
\text { Format: } 1 . \\
\text { Treatment Providers: } \\
1 . \\
\text { Dose: } \\
\text { Amount: Not } \\
\text { described. } \\
\text { Frequency: } \\
\text { once/week } \\
\text { Duration: } 4 \text {-weeks. } \\
\text { Heterogeneity: } 1 ; 4 \text {. } \\
\text { Approach: } 1 .\end{array}$ & \\
\hline $\begin{array}{l}\text { Study ID: 20; Medline } \\
\text { Review Author ID: } 1 \\
\text { Citation: } \\
\text { Eklund, K., Wilhelmson, K., } \\
\text { Gustafsson, H., Landahl, S., } \\
\text { Dahlin-Ivanoff, S., } \\
\text { Institutionen för } \\
\text { hälsovetenskaper, .. Lunds } \\
\text { universitet. (2013). One-year } \\
\text { outcome of frailty indicators } \\
\text { and activities of daily living } \\
\text { following the randomised } \\
\text { controlled trial: "continuum } \\
\text { of care for frail older people". } \\
\text { BMC Geriatrics, 13, 76. } \\
\text { doi:10.1186/1471-2318-13-76 } \\
\text { Date of Study: 2013. }\end{array}$ & $\begin{array}{l}\text { Purpose: } \\
\text { Evaluating the } \\
\text { effects of the } \\
\text { intervention on } \\
\text { functional ability in } \\
\text { terms } \\
\text { of activities of daily } \\
\text { living and frailty up to } \\
\text { one year } \\
\text { later. } \\
\text { Study Design: } 3 \text {. } \\
\text { Quality Rating: } 3 \text {. } \\
\text { Global Rating (for } \\
\text { weak/moderate } \\
\text { articles): } 1 ; 4 \text {. } \\
\text { N = 161 } \\
\text { Setting: } 0 \text {. } \\
\text { Country: } 3 \text {. } \\
\text { Medical Diagnosis: } 0 \text {. } \\
\text { Age: } 65+\end{array}$ & $\begin{array}{l}\text { Total number of } \\
\text { intervention } \\
\text { groups: Two. } \\
\text { number of } \\
\text { participants } \\
\text { allocated to each } \\
\text { intervention group: } \\
\text { Control: } 76 \\
\text { Intervention: } 85 \\
\\
\text { Outcomes and time } \\
\text { points collected and } \\
\text { reported: } \\
\text { Baseline data (= } \\
\text { interviews and } \\
\text { assessments) were } \\
\text { predominantly } \\
\text { collected within a } \\
\text { week following } \\
\text { discharge. Follow- } \\
\text { ups at three-, six- and } \\
12 \text { months. } \\
\text { Frailty (2) } \\
\text { Activities of Daily } \\
\text { Living (2) }\end{array}$ & $\begin{array}{l}\text { Measurement Tools: } \\
\text { Berg balance scale } \\
\text { KM chart } \\
\text { Mini Mental State } \\
\text { Examination } \\
\text { Hand dynamometer } \\
\text { ADL staircare } \\
\text { Reliability/validity } \\
\text { reported? } 1 .\end{array}$ & $\begin{array}{l}\text { Component } \\
\text { Number: two } \\
\text { Type: } 3 \\
\text { (Organizational/supp } \\
\text { ortive care) } \\
\text { Mode } \\
\text { Medium: } 1 \text {. } \\
\text { Format: } 2 \text {. } \\
\text { Treatment Providers: } \\
\text { 3. } \\
\text { Dose } \\
\text { Amount: Unclear } \\
\text { Frequency: within } \\
\text { one-week post- } \\
\text { discharge, then } \\
\text { once/month } \\
\text { Duration: at least } \\
\text { one year. } \\
\text { Heterogeneity: } 1 ; 4 \text {. } \\
\text { Approach: } 3\end{array}$ & $\begin{array}{l}\text { Indicator of } \\
\text { quality used: } \\
1 . \\
\text { Study Findings: } \\
\text { Outcome \#1: } 2 \\
\text { Statistical } \\
\text { significance: } \\
\text { Intervention: } 00 \\
\text { Control: } 00 \\
\text { Outcome \#2: } 2 \\
\text { Statistical } \\
\text { significance: } \\
\text { (3+ 12-month } \\
\text { follow-ups): } \\
\text { Intervention: } 1 \\
\text { Control: } 00 \text {. }\end{array}$ \\
\hline $\begin{array}{l}\text { Study ID: 21; Medline } \\
\text { Review Author ID: } 1 \\
\text { Citation: } \\
\text { Koehler, B. E., Richter, K. } \\
\text { M., Youngblood, L., Cohen, } \\
\text { B. A., Prengler, I. D., Cheng, } \\
\text { D., \& Masica, A. L. (2009). } \\
\text { Reduction of 30-day } \\
\text { postdischarge hospital } \\
\text { readmission or emergency } \\
\text { department (ED) visit rates in } \\
\text { high-risk elderly medical } \\
\text { patients through delivery of a } \\
\text { targeted care bundle. Journal } \\
\text { of Hospital Medicine, 4(4), } \\
211-218 . \\
\text { doi:10.1002/jhm.427 }\end{array}$ & $\begin{array}{l}\text { Purpose: } \\
\text { To assess the impact of } \\
\text { a supplemental care } \\
\text { bundle targeting high- } \\
\text { risk elderly inpatients } \\
\text { implemented by } \\
\text { hospital-based staff } \\
\text { compared to usual care } \\
\text { on a composite outcome } \\
\text { of hospital readmission } \\
\text { and/or ED visitation at } \\
30 \text { and } 60 \text { days } \\
\text { following discharge. } \\
\text { Study Design: } 1 . \\
\text { Quality Rating: } 2 \text {. } \\
\text { Global Rating (for } \\
\text { weak/moderate } \\
\text { articles): } 4 \text {. } \\
\mathrm{N}=41\end{array}$ & $\begin{array}{l}\text { Total number of } \\
\text { intervention } \\
\text { groups: Two } \\
\text { number of } \\
\text { participants } \\
\text { allocated to each } \\
\text { intervention group: } \\
21 \text { controls, } \\
20 \text { interventions } \\
\text { Outcomes and time } \\
\text { points collected and } \\
\text { reported: } \\
\text { Unplanned hospital } \\
\text { readmission or ED } \\
\text { visitation (1) at } 30- \\
\text { and } 60 \text {-days post } \\
\text { discharge were } \\
\text { collected via } \\
\text { BUMC's electronic }\end{array}$ & None. & $\begin{array}{l}\text { Component } \\
\text { Number: Four } \\
\text { Type: } 3 \text { (Medication } \\
\text { management/educati } \\
\text { on/supportive } \\
\text { care/organization) } \\
\text { Mode } \\
\text { Medium: } 4 \\
\text { (telephone } \\
\text { contact/face-to- } \\
\text { face/written } \\
\text { information). } \\
\text { Format: } 1 . \\
\text { Treatment } \\
\text { Provider: } 3 \text {. } \\
\text { Dose } \\
\text { Amount: Unclear. } \\
\text { Frequency: once (5- }\end{array}$ & $\begin{array}{l}\text { Indicator of } \\
\text { quality used: } 2 . \\
\text { Study Findings: } \\
\text { Outcome\# } 1: 1 \\
\text { Statistical } \\
\text { significance: } \\
\text { (30-days) } \\
\text { Intervention: } 1 \\
(\mathrm{P}=0.04) \\
\text { Control: } 00 . \\
\text { (60-days): } \\
\text { Intervention: } 00 \\
\text { (P = 0.52) } \\
\text { Control: } 00 .\end{array}$ \\
\hline
\end{tabular}




\begin{tabular}{|c|c|c|c|c|c|}
\hline Date of Study: 2009 & $\begin{array}{l}\text { Setting: } 5 . \\
\text { Country: } 1 . \\
\text { Medical Diagnosis; } 0 . \\
\text { Age: } 70\end{array}$ & $\begin{array}{l}\text { reporting systems } \\
\text { (30 and } 60 \text { days) }\end{array}$ & & $\begin{array}{l}7 \text { days post- } \\
\text { discharge). } \\
\text { Duration: } 5-7 \text { days } \\
\text { following discharge. } \\
\text { Heterogeneity: } 1 ; 4 \text {. } \\
\text { Approach: } 1 \text {. }\end{array}$ & \\
\hline $\begin{array}{l}\text { Study ID: 22; Medline } \\
\text { Review Author ID: } 1 \\
\text { Citation: } \\
\text { Saleh, S. S., Freire, C., } \\
\text { Morris-Dickinson, G., \& } \\
\text { Shannon, T. (2012). An } \\
\text { effectiveness and cost-benefit } \\
\text { analysis of a hospital-based } \\
\text { discharge transition program } \\
\text { for elderly medicare } \\
\text { recipients. Journal of the } \\
\text { American Geriatrics Society, } \\
\text { 60(6), 1051-1056. } \\
\text { doi:10.1111/j.1532- } \\
\text { 5415.2012.03992.x } \\
\text { Date of Study: } 2012\end{array}$ & $\begin{array}{l}\text { Purpose: } \\
\text { To investigate the } \\
\text { business case of post- } \\
\text { discharge care transition } \\
\text { (PDCT) among } \\
\text { Medicare beneficiaries } \\
\text { by conducting a cost- } \\
\text { benefit analysis. } \\
\text { Study Design: } 1 . \\
\text { Quality Rating: } 2 \text {. } \\
\text { Global Rating (for } \\
\text { weak/moderate } \\
\text { articles): } 5 \text {. } \\
\text { N = } 333 \\
\text { Setting: } 0 \text {. } \\
\text { Country: } 1 \text {. } \\
\text { Medical Diagnosis: } 0 . \\
\text { Age: } 65+\end{array}$ & $\begin{array}{l}\text { Total number of } \\
\text { intervention } \\
\text { groups: Two } \\
\text { number of } \\
\text { participants } \\
\text { allocated to each } \\
\text { intervention group: } \\
173 \text { control } \\
160 \text { intervention. } \\
\text { Outcomes and time } \\
\text { points collected and } \\
\text { reported: } \\
\text { Self-management } \\
\text { skills and abilities } \\
\text { (perceptions of) (3) } \\
\text { Readmission rates } \\
\text { (1) } \\
\text { Data were collected } \\
\text { at discharge } \\
\text { (baseline) and after } 6 \\
\text { weeks. }\end{array}$ & $\begin{array}{l}\text { Measurement Tools: } \\
\text { 15-item version of } \\
\text { Coleman's Care Transitions } \\
\text { Measure survey. } \\
\text { Reliability/validity } \\
\text { reported? } 1 .\end{array}$ & $\begin{array}{l}\text { Component } \\
\text { Number: four. } \\
\text { Type: } 3 \\
\text { (Organizational/medi } \\
\text { cation } \\
\text { management/supporti } \\
\text { ve care/educational). } \\
\text { Mode } \\
\text { Medium: } 1 . \\
\text { Format: } 2 \text {. } \\
\text { Treatment } \\
\text { Provider: } 3 \text {. } \\
\text { Dose } \\
\text { Amount: unclear. } \\
\text { Frequency: unclear. } \\
\text { Three home visits } \\
\text { (not specified), } \\
\text { follow-up with } \\
\text { physician within } 7 \\
\text { days of acute care } \\
\text { discharge. } \\
\text { Duration: } 45 \text { days } \\
\text { post discharge. } \\
\text { Heterogeneity: } 1 ; 4 \text {. } \\
\text { Approach: } 1 .\end{array}$ & $\begin{array}{l}\text { Indicator of } \\
\text { quality used: } 3 \text {. } \\
\text { Study Findings: } \\
\text { Outcome \#1: } 3 \\
\text { Statistically } \\
\text { Significant: } \\
\text { Intervention: } 1 \\
\text { How to manage } \\
\text { their health (P = } \\
.003 \text { ) } \\
\text { Understanding the } \\
\text { warning symptoms } \\
\text { and signs patients } \\
\text { should } \\
\text { watch for given } \\
\text { their health } \\
\text { conditions ( } \mathrm{P}= \\
.004) \text {, } \\
\text { Understanding the } \\
\text { written plan that } \\
\text { describes how } \\
\text { healthcare } \\
\text { plans are going to } \\
\text { be met ( } \mathrm{P}=.01 \text { ) } \\
\text { Confidence in terms } \\
\text { of } \\
\text { knowing what to do } \\
\text { to manage their } \\
\text { health (P = .03) } \\
\text { Being able to do the } \\
\text { things that they } \\
\text { need to take care of } \\
\text { their health ( } \mathrm{P}= \\
.03 \text { ) } \\
\text { Better } \\
\text { understanding of the } \\
\text { purpose of taking } \\
\text { their medications (P } \\
=.008 \text { ). } \\
\text { Control group: } 00 . \\
\text { Outcome \#2: } 1 \\
\text { Statistical } \\
\text { significance: } \\
\text { Intervention: } 00 . \\
\text { Control: } 00 \text {. }\end{array}$ \\
\hline $\begin{array}{l}\text { Study ID: 23; Medline } \\
\text { (Search \#2 home health care) } \\
\text { Review Author ID: } 1 \\
\text { Citation: } \\
\text { Stevens-Lapsley, J. E., Loyd, } \\
\text { B. J., Falvey, J. R., Figiel, G. }\end{array}$ & $\begin{array}{l}\text { Purpose: } \\
\text { To determine whether a } \\
\text { progressive } \\
\text { multicomponent } \\
\text { physical therapy } \\
\text { intervention in the } \\
\text { home setting can } \\
\text { improve functional }\end{array}$ & $\begin{array}{l}\text { Total number of } \\
\text { intervention } \\
\text { groups: Two. } \\
\text { number of } \\
\text { participants } \\
\text { allocated to each } \\
\text { intervention group: } \\
\text { Intervention: } \mathbf{n}=12\end{array}$ & $\begin{array}{l}\text { Measurement Tools: } \\
\text { 4-meter gait speed } \\
\text { assessment, the modified } \\
\text { Physical Performance Test, } \\
\text { the Short Physical } \\
\text { Performance Battery, and } \\
\text { the 6-minute walk } \\
\text { Test. }\end{array}$ & $\begin{array}{l}\text { Component } \\
\text { Number: Two } \\
\text { Type: } 3 \text { (Supportive } \\
\text { care, educational) } \\
\text { Mode } \\
\text { Medium: } 1 . \\
\text { Format: } 1 .\end{array}$ & $\begin{array}{l}\text { Indicator of } \\
\text { quality used: } 3 \text {. } \\
\text { Study Findings: } \\
\text { Outcome \#1: } 2 \\
\text { Statistical } \\
\text { significance: } \\
\text { Intervention: } 1\end{array}$ \\
\hline
\end{tabular}




\begin{tabular}{|c|c|c|c|c|c|}
\hline $\begin{array}{l}\text { J., Kittelson, A. J., Cumbler, } \\
\text { E. U., \& Mangione, K. K. } \\
\text { (2016). Progressive multi- } \\
\text { component home-based } \\
\text { physical therapy for } \\
\text { deconditioned older adults } \\
\text { following acute } \\
\text { hospitalization: A pilot } \\
\text { randomized controlled trial. } \\
\text { Clinical Rehabilitation, } 30(8) \text {, } \\
776-785 \text {. } \\
\text { doi:10.1177/02692155156032 } \\
19 \\
\text { Date of Study: } 2016 .\end{array}$ & $\begin{array}{l}\text { mobility for } \\
\text { deconditioned older } \\
\text { adults following acute } \\
\text { hospitalization. } \\
\text { Study Design: } 1 . \\
\text { Quality Rating: } 2 . \\
\text { Global Rating (for } \\
\text { weak/moderate } \\
\text { articles): } 4 \text {. } \\
\mathrm{N}=22 \\
\text { Setting: } 4 \text {. } \\
\text { Country: } 1 \text {. } \\
\text { Medical Diagnosis; } 0 . \\
\text { Age: } 65+\end{array}$ & $\begin{array}{l}\text { Control: } \mathbf{n}=10 \\
\text { Outcomes and time } \\
\text { points collected and } \\
\text { reported: } \\
\text { Baseline (within } \\
\text { 72hours of hospital } \\
\text { discharge), 30days, } \\
\text { and } \\
\text { 60days (primary end- } \\
\text { point) after } \\
\text { hospitalization. } \\
\text { Physical } \\
\text { performance (2), 4- } \\
\text { meter gait speed, } \\
\text { incidence of adverse } \\
\text { events (re- } \\
\text { hospitalizations and } \\
\text { emergency room } \\
\text { visits). (1) }\end{array}$ & $\begin{array}{l}\text { Reliability/validity } \\
\text { reported? } \\
1 .\end{array}$ & $\begin{array}{l}\text { Treatment } \\
\text { Provider: } 4 \\
\text { (physiotherapist). } \\
\text { Dose } \\
\text { Amount: not } \\
\text { described. } \\
\text { Frequency: 2- } \\
\text { 3/week; } 9.67 \text { visits } \\
\text { total } \\
\text { Duration: Average } \\
\text { of } 30.58 \text { days. } \\
\text { Heterogeneity: } 1 ; 4 \text {. } \\
\text { Approach: } 1 .\end{array}$ & $\begin{array}{l}\text { (gait speed, } \\
\text { modified Physical } \\
\text { Performance Test } \\
\text { scores, and Short } \\
\text { Physical } \\
\text { Performance } \\
\text { Battery scores). } \\
\text { Control: } 00 . \\
\text { Outcome \#2: } 1 \\
\text { Statistical } \\
\text { significance: } \\
\text { Intervention: } 00 . \\
\text { Control: } 00 \text {. }\end{array}$ \\
\hline
\end{tabular}


Study ID: 24; Medline

Review Author ID: 1

\section{Citation:}

Courtney, M. D., Edwards, H. E., Chang, A. M., Parker, A. W., Finlayson, K., \& Hamilton, K. (2011). A randomised controlled trial to prevent hospital readmissions and loss of functional ability in high risk older adults: A study protocol. BMC Health Services Research, 11(1), 202-202. doi:10.1186/14726963-11-202

Results found here: Finlayson, K., Chang, A. M., Courtney, M. D., Edwards, H. E., Parker, A. W., Hamilton, K., . . . O'Brien, J. (2018). Transitional care interventions reduce unplanned hospital readmissions in high-risk older adults. BMC Health Services Research, 18(1), 956. doi:10.1186/s12913018-3771-9

Date of Study: 2011.
Purpose:

To evaluate the

relative

effectiveness of

transitional care

strategies commencing

during hospitalization

for community-based

high risk

older adults on

emergency

readmissions and

health service use,

functional ability and

quality of life

outcomes.

Study Design: 3 .

Quality Rating: 2.

Global Rating (for

weak/moderate

articles): 1 .

$\mathrm{N}=168$

Setting: 1 .

Country: 4 .

Medical Diagnosis: 0 .

Age: $65+$
Total number of intervention groups:

Four groups: 1) the usual care control group, 2) the exercise and

in-home/telephone

follow-up intervention

group, 3) the

exercise only

intervention group, or 4)

the in-home/telephone

follow-up only

intervention group.

number of participants

allocated to each

intervention group:

(control group)

55 in the control group.

(Intervention group 1)

56 in the exercise only

intervention group.

(Intervention group 2)

57 in the exercise and

follow-up intervention

group.

(Intervention group 3)

54 in the home/telephone

intervention group.

Outcomes and time points collected and reported:

Baseline, 28 days, 12

weeks and 24 weeks.

Primary Outcomes:

Emergency health

service use (i.e.

unplanned readmissions, time to first unplanned

readmission, unplanned

Emergency Department,

General Practitioner and other health service use) (1).
Measurement Tools:

Functional ability

(Instrumental Activities

of Daily Living, Index

of Activities of Daily

Living, Walking

Impairment

Questionnaire).

Health-related quality

of life (Short Form-

12v2 Survey),

psychosocial well-being

(Geriatric Depression

Scale, MOS Social

Support

Survey).

Reliability/validity reported? 1 .
Intervention Group \#1:

Exercise only follow-up

intervention group:

Component Number: one.

Type: 2 .

\section{Mode}

Medium: 4 (face-to-

face/written).

Format: 1 .

Treatment Providers: 4.

$\underline{\text { Dose }}$

Amount: Not

described.

Frequency: 72 hours

after admission, 6

weekly in-home visits.

Duration: 24 weeks

after discharge.

Heterogeneity: 1;4.

Approach: 1.

Intervention Group \#2:

Exercise and in-

home/telephone follow-

up intervention group:

Component Number:

Two.

Type: 3

(organizational,

supportive care)

Mode

Medium: 4 (face-toface/written/telephone follow-up).

Format: 2.

Treatment Providers: 3.

Dose

Amount: Not

described.

Frequency: 72 hours after admission, every 2 days while in hospital; 48 hours postdischarge, 6 weekly inhome visits; telephone calls weekly for first 4weeks post-discharge, than monthly for 6 months following discharge from hospital. Duration: 24 weeks after discharge.

Heterogeneity: 2; 3

Approach: 3 .
Indicator of quality used: 2 .

Study Findings:

Outcome \#1: 1

28 days

Statistical

significance: Group

1: 00

Group 2: $1(\mathrm{p}=$

0.029 )

Group 3: 1

$(\mathrm{p}=0.067)$

Control Group: 00.

12- weeks:

Statistical

significance: Group 1: 00

Group 2: $1(\mathrm{p}=$

0.014 )

Group 3: 1

$(\mathrm{p}=0.040)$

Control Group: 00.

24- weeks after

discharge:

Statistical

Significance: Group 1:00.

Group 2: 00

Group 3: 00.

Control Group: 00. 
Intervention Group \#3:

In-home/telephone

follow-up only

intervention group

Component Number:

Two.

Type: 3

(organizational/supporti ve care).

Mode

Medium: 4 (face-to-

face/written/telephone

follow-up).

Format: 2 .

Treatment Providers: 1.

Dose

Amount: Not

described.

Frequency: 72 hours

after admission, every 2

days while in hospital,

within 48 hours post-

discharge, weekly for

4-weeks post-discharge,

monthly for 6 months

post-discharge.

Duration: 24 weeks.

Heterogeneity: 2;3.

Approach: 3 . 


Study ID: 25; Medline
Search Number 3
Review Author ID: 1
Citation:
Basger, B. J., Moles, R. J.,
\& Chen, T. F. (2015).
Impact of an enhanced
pharmacy discharge service
on prescribing
appropriateness criteria: A
randomised controlled trial.
International Journal of
Clinical Pharmacy, 37(6),
1194-1205.
doi:10.1007/s11096-015-
0186-0

Date of Study: 2015.

\section{Purpose:}

To assess the impact

of applying our

criteria-set, during medication review, on change in the number of criteria met and on health related QoL, as part of an enhanced pharmacy discharge service

Study Design: 3 . Quality Rating: 2. Global Rating (for weak/moderate articles): 4 .

$\mathrm{N}=216$

Setting: 0 .

Country: 4 .

Medical Diagnosis; 0. Age: $65+$

(

Study ID: 26; Medline

Review Author ID: 1

\section{Citation:}

Gurwitz, J. H., Field, T. S., Ogarek, J., Tjia, J., Cutrona, S. L., Harrold, L. R., . . . Garber, L. (2014). An electronic health recordbased intervention to increase follow-up office visits and decrease rehospitalization in older adults. Journal of the American Geriatrics Society, 62(5), 865-871. doi:10.1111/jgs.12798

Date of Study: 2014.

\section{Purpose:}

To assess the effect of an electronic health record-based transitional care intervention involving automated alerts to primary care providers and staff when older adults were discharged from the hospital.

Study Design: 3 . Quality Rating: 1. Global Rating (for weak/moderate articles): $1 ; 4$.

$\mathrm{N}=3661$

Setting: 0 .

Country: 1.

Medical Diagnosis; 0.

Age: $65+$

\section{Total number of intervention groups: \\ Two. \\ number of participants \\ Measurement Tools: \\ The RAND Short \\ Form 36 (SF-36)}

allocated to each

intervention group:

Control: $\mathrm{n}=102$

Intervention: n- 114

Outcomes and time points collected and reported:

3- months post-discharge.

Main outcome measures:

change in HRQoL (3);

number and causes of

Drug Related Problems

identified by medication

review; intervention

patient medication

recommendation

implementation rates (2).
Reliability/validity reported? 1.
Component Number:

Total number of

intervention groups:

Two

Number of participants

allocated to each

intervention group:

Intervention: $\mathbf{n}=1870$

Control: $\mathbf{n = 1 7 9 1}$

Outcomes and time points collected and reported:

Whether discharged individuals had an office visit with a primary care physician (1) in the 7-, 14, and 30-day periods after hospital discharge was determined, as was whether a participant was re-hospitalized within 30 days.

Type: 3 (supportive post-discharge; oneone.

Type: 3 (Medication

management)

\section{Mode}

Medium: 4 (face-toface, written material).

Format: 1 .

Treatment Provider: 4 (pharmacist).

$\underline{\text { Dose }}$

Amount: Not

described.

Frequency: Not

described.

Duration: Not

described.

Heterogeneity: Not

described.

Approach: 1.

Indicator of quality used: 3 .

Study Findings:

Outcome \#1: 3

Intervention: 00.

Control: 00.

Outcome \#2: 2

Statistical significance: Intervention: 00 . Control: 00.

Indicator of quality used: 2 . care/medication management).

\section{Mode}

Medium: 3

(electronic).

Format: 1 .

Treatment Provider: 4 (Online).

Dose

Amount: Not reported. Frequency: 3-days week post-discharge Duration: one week. Heterogeneity: 1;4.

Approach: 1.
Study Findings:

Outcome \#1: 1

Statistical significance: Intervention: 00. Control: 00. 


\begin{tabular}{|c|c|c|c|c|c|}
\hline Stbewudy ID: 27; Medline & $\begin{array}{l}\text { Purpose: } \\
\text { To compare the effect } \\
\text { of high-intensity } \\
\text { aerobic interval } \\
\text { exercise (HIA) with } \\
\text { home-based exercise } \\
\text { (HB) in older adults } \\
\text { with chronic disease } \\
\text { soon after } \\
\text { discharge from the } \\
\text { hospital. } \\
\text { Study Design: } 3 \text {. } \\
\text { Quality Rating: } 3 \text {. } \\
\text { N = 115 } \\
\text { Setting: } 1 \text {. } \\
\text { Country: } 3 \text {. } \\
\text { Medical Diagnosis; } 0 \text {. } \\
\text { Age: } 70+\end{array}$ & $\begin{array}{l}\text { Total number of } \\
\text { intervention groups: } \\
\text { Two. } \\
\text { number of participants } \\
\text { allocated to each } \\
\text { intervention group: } \\
\text { Intervention group 1: } \\
\text { High-intensity } \\
\text { aerobic interval exercise } \\
\text { (HIA): (59) } \\
\text { Intervention group 2: } \\
\text { Home-based exercise } \\
\text { (HB): (56) } \\
\text { Outcomes and time } \\
\text { points collected and } \\
\text { reported: } \\
\text { 2-4 weeks post-discharge } \\
\text { and again after 12-weeks. } \\
\text { Health-related quality of } \\
\text { life (3). } \\
\text { Senior Fitness Test (2) }\end{array}$ & $\begin{array}{l}\text { Measurement Tools: } \\
\text { Medical Outcomes } \\
\text { Study 36-item Short } \\
\text { Form Survey. } \\
\text { Senior Fitness Test. } \\
\text { Physical Activity } \\
\text { Scale for the Elderly } \\
\text { (PASE). } \\
\text { Reliability/validity: } 1 .\end{array}$ & $\begin{array}{l}\frac{\text { I High-Intensity }}{\text { Aerobic Exercise: }} \\
\text { Component Number: } \\
\text { One. } \\
\text { Type: } 2 . \\
\text { Mode } \\
\text { Medium: } 1 . \\
\text { Format: } 1 \text {. } \\
\text { Treatment Provider: } \\
\text { 4. } \\
\text { Dose } \\
\text { Amount: } 60 \text { minutes. } \\
\text { Frequency: } 2 x / \text { week } \\
\text { while in hospital; } \\
\text { 1/week post-discharge. } \\
\text { Duration: } 3 \text { months. } \\
\text { Heterogeneity: } 2 ; 4 \text {. } \\
\text { Approach: } 1 .\end{array}$ & $\begin{array}{l}\text { Indicator of } \\
\text { quality used: } 1 . \\
\text { Study Findings: } \\
\text { Outcome \#1: } 2 \\
\text { Intervention } 1: 1 \\
\text { Senior Fitness Test } \\
\text { (chair stand (p= } \\
0.004 \text { ) Arm curl } \\
\text { (0.001) } \\
\text { 6-minute walk } \\
\text { (0.001) } \\
\\
\text { Outcome \#2: } 3 \\
\text { Statistical } \\
\text { significance: } \\
\text { Intervention } 1: 00 .\end{array}$ \\
\hline $\begin{array}{l}\text { Study ID: 28; Medline } \\
\text { Review Author ID: } 1 \\
\text { Citation: } \\
\text { Vogler, Constance M., } \\
\text { MBBS, FRACP, PhD, } \\
\text { Menant, J. C., PhD, } \\
\text { Sherrington, C., PhD, Ogle, } \\
\text { Susan J., MBBS, FRACP, \& } \\
\text { Lord, Stephen R., PhD, DSc. } \\
\text { (2012). Evidence of } \\
\text { detraining after 12-week } \\
\text { home-based exercise } \\
\text { programs designed to reduce } \\
\text { fall-risk factors in older } \\
\text { people recently discharged } \\
\text { from hospital. Archives of } \\
\text { Physical Medicine and } \\
\text { Rehabilitation, 93(10), } \\
\text { 1685-1691. } \\
\text { doi:10.1016/j.apmr.2012.03. } \\
\text { 033 }\end{array}$ & $\begin{array}{l}\text { Purpose: } \\
\text { To measure the extent } \\
\text { to which improved } \\
\text { sensorimotor function } \\
\text { and balance resulting } \\
\text { from a } 12 \text {-week } \\
\text { exercise intervention } \\
\text { were retained } 12 \\
\text { weeks after exercise } \\
\text { cessation in } \\
\text { older adults recently } \\
\text { discharged from } \\
\text { hospital. } \\
\text { Study Design: } 3 \text {. } \\
\text { Quality Rating: } 3 \text {. } \\
\text { N = } 180 \\
\text { Setting: } 5 \text {. } \\
\text { Country: } 4 \text {. } \\
\text { Medical Diagnosis; } 0 \text {. } \\
\text { Age: } 65+\end{array}$ & $\begin{array}{l}\text { Total number of } \\
\text { intervention groups: } \\
\text { Three } \\
\text { Number of participants } \\
\text { allocated to each } \\
\text { intervention group: } \\
\text { (intervention group 1) } \\
\text { Seated Strengthening } \\
\text { Training (n=60) } \\
\text { (intervention group 2) } \\
\text { Weight-bearing } \\
\text { Strengthening Training } \\
\text { (n=60) } \\
\text { (Intervention group 3) } \\
\text { Social Visits (n= 60) } \\
\text { Outcomes and time } \\
\text { points collected and } \\
\text { reported: } \\
\text { Physical status (2): } \\
\text { Physiological Profile } \\
\text { Assessment (PPA), a } \\
\text { composite sensorimotor } \\
\text { fall-risk score, and 2 } \\
\text { measures of controlled } \\
\text { leaning balance assessed } \\
\text { at baseline. } \\
\text { Immediately after } \\
\text { intervention; 12 weeks } \\
\text { later. }\end{array}$ & $\begin{array}{l}\text { Measurement Tools: } \\
\text { Physiological Profile } \\
\text { Assessment (PPA) } \\
\text { composite fall-risk } \\
\text { score } \\
\text { Melbourne Edge Test } \\
\text { Reliability/validity } \\
\text { Reported? } 1 \text {. }\end{array}$ & $\begin{array}{l}\text { Intervention Group \#1: } \\
\text { Component Number: } \\
\text { two. } \\
\text { Type: } 3 \text { (education and } \\
\text { supportive care). } \\
\text { Mode } \\
\text { Medium: } 1 . \\
\text { Format: } 1 . \\
\text { Treatment Providers: } 4 \text {. } \\
\text { Dose } \\
\text { Amount: unclear. } \\
\text { Frequency: } 8 \text { visits. } \\
\text { Duration: } 12 \text { weeks. } \\
\text { Heterogeneity: Not } \\
\text { reported. } \\
\text { Approach: } 1 \text {. } \\
\text { Intervention Group \#2: } \\
\text { Component Number: } \\
2 \\
\text { Type: } 3 \text { (Education and } \\
\text { supportive care). } \\
\text { Mode } \\
\text { Medium: } 1 . \\
\text { Format: } 1 \text {. } \\
\text { Treatment Providers: } 4 \text {. } \\
\text { Dose } \\
\text { Amount: unclear. } \\
\text { Frequency: } 8 \text { visits. } \\
\text { Duration: } 12 \text { weeks. } \\
\text { Heterogeneity: Not } \\
\text { reported. } \\
\text { Approach: } 1 . \\
\text { Intervention Group \#3: }\end{array}$ & $\begin{array}{l}\text { Indicator of } \\
\text { quality used: } 1 . \\
\text { Study Findings: } \\
\text { Outcome \#1: } 2 \\
\text { Statistical } \\
\text { significance: } \\
\text { Intervention group } \\
1: 00 . \\
\text { Intervention group } \\
2: 00 \\
\text { Control: } 00 \text {. }\end{array}$ \\
\hline
\end{tabular}


Study ID: 29; Medline

Review Author ID: 1

\section{Citation:}

Rytter, L., Jakobsen, H. N., Rønholt, F., Hammer, A. V., Andreasen, A. H., Nissen, A., \& Kjellberg, J. (2010). Comprehensive discharge follow-up in patients' homes by GPs and district nurses of elderly patients. A randomized controlled trial. Scandinavian Journal of Primary Health Care, 28(3), 146-153.

doi:10.3109/0281343100376 4466

Date of Study: 2010

Study ID: 30; Medline

\section{Review Author ID: 1}

\section{Citation:}

Ueda, T., Higuchi, Y., Imaoka, M., Todo, E., Kitagawa, T., \& Ando, S. (2017). Tailored education program using home floor plans for falls prevention in discharged older patients: A pilot randomized controlled trial. Archives of Gerontology and Geriatrics,

\section{Purpose:}

To assess whether a follow-up program undertaken by GPs and district nurses could improve the quality of the medical treatment and reduce the risk of readmission of elderly newly discharged patients.

Study Design: 3 . Quality Rating: 2. Global Rating (for weak/moderate articles): 4 .

$\mathrm{N}=333$

Setting: 5 .

Country: 3 .

Medical Diagnosis: 0. Age: $78+$

\section{Purpose:}

To investigate the effect of a tailored education program using home floor plans on falls prevention in discharged older patients.

Study Design: 1 . Quality Rating: 3 . $\mathrm{N}=51$

Setting: 0 .

Country: 2 .

Medical Diagnosis; 0. Age: 65+

\section{Total number of} intervention groups: Two number of participants allocated to each intervention group: Intervention group (166) Control group (165)

Outcomes and time points collected and reported:

Primary outcomes: hospital readmission (within 26 weeks postdischarge) (1) and the concordance between the GP's knowledge of the medical treatment and what the patient was actually taking (2).

12 weeks after discharge, a structured interview guided by a questionnaire was conducted.

\section{Total number of} intervention groups: Two number of participants allocated to each intervention group: Intervention: $(\mathbf{n}=25)$ Control: $(\mathbf{n}=26)$

Outcomes and time points collected and reported:

Falls (2)

Follow-up one month after discharge.

\section{None.}

.

Measurement Tools: Timed Up and Go test Barthel Index Score Modified Fall Efficacy scale

Life Space Assessment

Reliability/validity reported? 1 . one.

Type: 2.

Mode

Medium: 1 .

Format: 1 .

Treatment Providers:

4 (social workers).

Dose

Amount: 60 minutes. Frequency: 8 visits, 2/month.

Duration: 3 months. Heterogeneity: Not reported.

Approach: 1. Component Number: Two

Type: 3 (supportive care, medication management)

\section{Mode}

Medium: 1.

Format: 1 .

Treatment Provider: 3 (physician/nurse).

Dose

Amount: 25, 30, 50 minutes.

Frequency: Three visits; 1 -week postdischarge, third- and eighth-week postdischarge.

Duration: 8 weeks.

Heterogeneity: 1;4.

Approach: 3.

Component Number:

One.

Type: 1 .

Mode

Medium: 1.

Format: 1 .

Treatment Provider: 4.

Dose

Amount: Not reported. Frequency: Not reported. Only during hospital stay.

Duration: Not reported.
Indicator of quality used: 3 .

Study

Findings/Statistical Significance:

Outcome \#1: 1

Statistical

Significance:

Intervention: 00 .

Control: 00.

Outcome \#2: 2

Statistical

significance:

Intervention: 1

Number of patients

with adjusted

medication since

discharge $(\mathrm{p}=0.01)$

Number of drugs

taken $(\mathrm{p}=0.0005)$

Control: 00 .

\section{Indicator of} quality used: 1 .

Study Findings:

Outcome \# 1: 2

Statistical

significance:

Intervention: 00.

Control: 00. 
doi:10.1016/j.archger.2017. 02.010

Date of Study: 2017 Study ID: 31; HealthStar Search \#1

Review Author ID: 1

\section{Citation:}

Wong, F. K., Ho, M. M., Yeung, S., Tam, S. K., \& Chow, S. K. (2011). Effects of a health-social partnership transitional program on hospital readmission: A randomized controlled trial. Social Science \& Medicine, 73(7), 960-969.

doi:10.1016/j.socscimed.201 1.06 .036

Date of Study: 2011

Study ID: 32; HealthStar\#3

Review Author ID: 1.

\section{Citation:}

Beelen, J., de Roos, N. M., \& de Groot, Lisette C P G M. (2017). A 12-week intervention with proteinenriched foods and drinks improved protein intake but not physical performance of older patients during the first 6 months after hospital release: A randomised controlled trial. The British Journal of Nutrition, 117(11), 1541-1549. doi:10.1017/S00071145170 01477

Date of Study: 2017
Purpose: To explore the outcomes of a health-social partnership program on postdischarge medical patients.

Study Design: 3 . Quality Rating: 3. $\mathrm{N}=686$

Setting: 1 .

Country: 2 . Age: 65+ allocated to each intervention group:

Intervention: 339

Control: 347

Outcomes and time points collected and reported:

(at discharge),
Data were collected at

\section{Total number of intervention groups: Two}

Number of participants

three time-points, at $\mathrm{O} 1$

O2 (4 weeks after

discharge and when the

HSTCMP was completed) and $\mathrm{O} 3$ (12 weeks after discharge).

The number of rehospitalizations (1) within 28 days (4 weeks) and 84

days (12 weeks) of

discharge, and days between index discharge and

readmission.

Purpose: The

effectiveness of a 12-

week intervention with protein-enriched foods and drinks by following-up seventyfive older patients during their first 6 months after hospital discharge.

Study Design: 3 . Quality Rating: 2. Global Rating (for weak/moderate articles): 4 . $\mathrm{N}=75$

Setting: 5 .

Country: 3 .

Medical Diagnosis: $0 . \quad$ The primary outcome for Age: 65+
Total number of intervention groups:

Two.

Number of participants allocated to each intervention group:

Control: 39 .

Intervention: 36 .

Outcomes and time points collected and reported:

Two primary outcomes: protein intake (2) and physical

performance (2) during the 12 -week intervention period. physical recovery was measured with the Short Physical Performance Battery (SPPB).

-balance, gait speed and chair-rise time.

Protein intake was measured at baseline
Measurement Tools:

Appropriateness

Evaluation Protocol.

MOS 36-item Short

Form Health Survey (SF-36).

Chronic Disease SelfEfficacy Scale.

Satisfaction with care (measured only at $\mathrm{O} 2$ ) 15-item questionnaire.

Reliability/validity reported? 1.

Measurement Tools:

Short Physical

Performance Battery (SPPB)

Physical recovery: gait speed in seconds (measured within the SPPB), chair-rise time (in sec) (measured within the SPPB), legextension strength, hand-grip strength, body weight, nutritional status (Mini

Nutritional

Assessment; MNA), independence in activities of daily living (using Barthel Index) and physical activity questionnaire. Handheld dynamometry: hand dynamometer

(Lafayette Instrument Company).

Reliability/validity
Heterogeneity: Not reported.

Approach: 2.

Component Number:

Two.

Type: 3

(organizational,

supportive care)

\section{Mode}

Medium: 4 (face-to-

face, telephone

contact).

Format: 1 .

Treatment Providers:

3 (nurse case manager, volunteers, social workers).

\section{Dose}

Amount: not reported Frequency: once during hospital admission; once/week post-discharge.

Duration: 4-weeks.

Heterogeneity: 1;4.

Approach: 3.

\section{Component Number:}

One.

Type: 2 .

\section{Mode}

Medium: 1.

Format: 1 .

Treatment Provider: 4.

Dose

Amount: Not reported.

Frequency: Began 2 days after admission, daily while in hospital, once post-discharge.

Duration: 12 weeks.

Heterogeneity: 1;4.

Approach: 3 .
Indicator of quality used: 3 .

Study

Findings/statistical significance:

Outcome \#1: 1

Statistical

significance:

Intervention: 1

The 28-day (4-

weeks) readmission

rates for $(\mathrm{p}=0.005)$.

Control: 00 .

84-day (12-weeks) readmission rates

( $\mathrm{p}=0.001)$.

Control: 00 .

The number of days between the index discharge and first readmission within 4 weeks ( $\mathrm{p}=$ 0.016).

Indicator of quality used: 1.

Study Findings:

Outcome \#1: 2

Statistical

significance:

Intervention: 1

consumption of

protein $(\mathrm{p}=<0.01)$.

Control: 00.

Outcome \#2: 2

Statistical

significance:

Intervention: 00.

Control: 00. 


\begin{tabular}{|c|c|c|c|c|c|}
\hline & & $\begin{array}{l}\text { (week } 0 \text { ) and at } 2,6 \text { and } \\
12 \text { weeks after hospital } \\
\text { discharge. } \\
\text { Physical performance was } \\
\text { measured at baseline } \\
\text { (week } 0 \text { ) and at } 2,6 \text { and } \\
12 \text { weeks after hospital } \\
\text { discharge. } \\
\text { For both protein intake } \\
\text { and physical performance, } \\
\text { a follow-up measurement } \\
\text { was taken at } 24 \text { weeks } \\
\text { after hospital discharge. }\end{array}$ & $\begin{array}{l}\text { reported? } \\
1 .\end{array}$ & & \\
\hline $\begin{array}{l}\text { Study ID: 33; HealthStar } \\
\text { Review Author ID: } 1 . \\
\text { Citation: } \\
\text { Di Monaco, M., De Toma, } \\
\text { E., Gardin, L., Giordano, S., } \\
\text { Castiglioni, C., \& Vallero, } \\
\text { F. (2015). A single } \\
\text { postdischarge telephone call } \\
\text { by an occupational therapist } \\
\text { does not reduce the risk of } \\
\text { falling in women after hip } \\
\text { fracture: A randomized } \\
\text { controlled trial. European } \\
\text { Journal of Physical and } \\
\text { Rehabilitation Medicine, } \\
\text { 51(1), } 15 . \\
\text { Date of Study: } 2015\end{array}$ & $\begin{array}{l}\text { Purpose: } \\
\text { To assess the } \\
\text { effectiveness of a } \\
\text { single telephone call } \\
\text { by an OT in reducing } \\
\text { the proportion of } \\
\text { fallers and improving } \\
\text { the adherence to fall } \\
\text { prevention strategies } \\
\text { after hospitalization } \\
\text { for hip-fracture. } \\
\text { Study Design: } 3 \text {. } \\
\text { Quality Rating: } 3 \text {. } \\
\text { N = } 169 \\
\text { Setting: } 0 \text {. } \\
\text { Country: } 3 \text {. } \\
\text { Medical Diagnosis; } 4 \text {. } \\
\text { Age: } 65+\end{array}$ & $\begin{array}{l}\text { Total number of } \\
\text { intervention groups: } \\
\text { Two. } \\
\text { number of participants } \\
\text { allocated to each } \\
\text { intervention group: } \\
\text { Intervention: } 78 \\
\text { Control: } 75 \\
\text { Outcomes and time } \\
\text { points collected and } \\
\text { reported: } \\
\text { 6- month follow-up } \\
\text { Primary outcome: Falls } \\
\text { (2). }\end{array}$ & $\begin{array}{l}\text { Measurement Tools: } \\
\text { Barthel Index Score. } \\
\text { Reliability/validity } \\
\text { reported? } 1 .\end{array}$ & $\begin{array}{l}\text { Component Number: } \\
\text { Three. } \\
\text { Type: } 3 \text { (medication } \\
\text { management, } \\
\text { supportive care, and } \\
\text { education). } \\
\text { Mode } \\
\text { Medium: } 4 \text { (written } \\
\text { and telephone). } \\
\text { Format: } 1 . \\
\text { Treatment Provider: } 4 \\
\text { (occupational } \\
\text { therapist). } \\
\text { Dose } \\
\text { Amount: } 35 \text { minutes. } \\
\text { Frequency: Once; } \\
\text { median of } 18 \text { days post- } \\
\text { discharge. } \\
\text { Duration: } 15-20 \text { days } \\
\text { post-discharge. } \\
\text { Heterogeneity: } 1 ; 4 \text {. } \\
\text { Approach: } 3 \text {. }\end{array}$ & $\begin{array}{l}\text { Indicator of } \\
\text { quality used: } 1 . \\
\text { Study Findings: } \\
\text { Outcome \#1: } 2 \\
\text { Statistical } \\
\text { significance: } \\
\text { Intervention: } 00 . \\
\text { Control: } 00 .\end{array}$ \\
\hline
\end{tabular}




\begin{abstract}
Study ID: 34; HealthStar
\end{abstract}
Review Author ID: 1.

\section{Citation:}

Pedersen, J. L., Pedersen, P. U., \& Damsgaard, E. M. (2016). Early nutritional follow-up after discharge prevents deterioration of ADL functions in malnourished, independent, geriatric patients who live alone-A randomized clinical trial. The Journal of Nutrition, Health \& Aging, 20(8), 845-853. doi:10.1007/s12603-0150629-2

Date of Study: 2016
Purpose: To compare the effects of two nutritional follow-up interventions strategies with no follow-up, with regard to preventing short-term deterioration in ADL, effect on physical function, health-related quality of life, and emotional health.

Study Design: 3 . Quality Rating: 3 . $\mathrm{N}=208$

Setting: 3 .

Country: 3 .

Medical Diagnosis; 2.

Age: $75+$

Total number of
intervention groups:
Three.
number of participants
allocated to each
intervention group:
Intervention $1:$
(home visit): 73
Intervention $2:$
(telephone
consultation): 68
Control: 67

Outcomes and time points collected and reported:

Follow-up at eight weeks.

Primary outcome: changes in ADL (2)
Measurement

Tools:

ADL (Barthel-100

score)

(handgrip strength,

30-sec. chair stand

test, CAS)

Quality of life and

depression

measurements (SF-

36, Depression List,

Geriatric Depression

Score)

Avlund mobility-

tiredness score (MobT)

Reliability/validity reported? 1 .
Study ID: 35; PsychInfo

Search \#1

Review Author ID: 1.

\section{Citation:}

Chow, S. K. Y., \& Wong, F. K. Y. (2014). A randomized controlled trial of a nurse-led case management programme for hospital-discharged older adults with co-morbidities. Journal of Advanced Nursing, 70(10), 2257-2271. doi:10.1111/jan.12375

Date of Study: 2014

\section{Purpose:}

To examine the effects of a nurse-led case management program for

hospital-discharged older adults with comorbidities.

Study Design: 3 . Quality Rating: 3 . $\mathrm{N}=281$

Setting: 1 .

Country: 2 .

Medical Diagnosis; 0. Age: $65+$

\section{Total number of} intervention groups:

Three.

number of participants allocated to each intervention group: Intervention group 1: Home visits: 87 Intervention group 2: Call group: 96 Control group: 98

Outcomes and time points collected and reported:

Time 1 (T1) was the baseline at the time of discharge, Time 2 (T2) was at 4 weeks after discharge when the interventions were completed and Time 3 (T3) was at 12 weeks. Primary Outcomes: unplanned readmission rates (1) (28 and 84-day readmissions).
Intervention group \#1

Home Visit

Component Number:

One.

Type: 1 .

Mode

Medium: 1.

Format: 2.

Treatment Provider: 4.

Dose

Amount: 45 minutes

Frequency: one, two,

four weeks after

discharge.

Duration: 4-weeks.

Heterogeneity: 1;4

Approach: 2 .

Intervention \#2:

Telephone Counselling

Component Number:

one.

Type: 1 .

Mode

Medium: 2 .

Format: 2 .

Treatment Provider:

4.

Dose

Amount: 15 minutes. Frequency: one, two, four weeks after discharge.

Duration: 4-weeks.

Heterogeneity: 1;4.

Approach: 2.

Home visit group:

Component Number:

Two

Type: 3

(organizational/

supportive care).

\section{Mode}

Medium: 4 (face-to-

face, telephone

contact).

Format: 1 .

Treatment Provider: 1 .

Dose

Amount: Not reported.

Frequency: 72 hours

post-discharge,

once/week

Duration: four weeks.

Heterogeneity: 1;4.

Approach: 3 .

Phone contact group:

Component Number:

two.

Type: 3

(organizational/

supportive care).
Indicator of

quality used: 1 .

Study

Findings/statistical

significance:

Outcome \#1: 2

Statistically

significant:

Intervention 1: 1

$(\mathrm{p}=<0.01)$.

Walking $(\mathrm{p}=0.01)$

Dressing $(\mathrm{p}=<0.01)$

Bladder control

$(\mathrm{p}=0.01)$.

Intervention 2: 00.

Control Group:00.

Indicator of

quality used: 2 .

Study

Findings/Statistical

Significance:

Outcome \#1: 1

Statistical

significance:

28 days post-

discharge

Intervention \#1: 00

Intervention \#2: 00

Control Group: 00

Statistical

significance:

84-days post-

discharge

Intervention \#1: 1

$(\mathrm{p}=0.059)$

Intervention \#2: 1

( $\mathrm{p}=0.007)$

Control Group: 00 
Mode

Medium: 2.

Format: 1 .

Treatment Provider:1.

Dose

Amount: not reported.

Frequency: once/week

Duration: four weeks.

Heterogeneity: 1;4.

Approach: 3 . 


\begin{abstract}
Study ID: 36; PsychInfo
\end{abstract}
Review Author ID: 1 .

\section{Citation:}

Courtney, M. D., Edwards, H. E., Chang, A. M., Parker, A. W., Finlayson, K., Bradbury, C., \& Nielsen, Z. (2012). Improved functional ability and independence in activities of daily living for older adults at high risk of hospital readmission: A randomized controlled trial. Journal of Evaluation in Clinical Practice, 18(1), 128.

Date of Study: 2011

\section{Study ID: 37; PsychInfo}

Review Author ID: 1.

\section{Citation:}

Toye, C., Parsons, R., Slatyer, S., Aoun, S. M., Moorin, R., Osseiran-Moisson, R., \& Hill, K. D. (2016). Outcomes for family carers of a nursedelivered hospital discharge intervention for older people (the further enabling care at home program): Single blind randomised controlled trial. International Journal of Nursing Studies, 64, 32-41. doi:10.1016/j.ijnurstu.2016.09 .012

Date of Study: 2016

Study ID: 38; Proquest

Search 2

\section{Review Author ID: 1}

\section{Citation:}

Haines, T. P., Russell, T., Brauer, S. G., Erwin, S., Lane, P., Urry, S., . . Condie, P. (2009). Effectiveness of a video-based exercise programme to reduce falls and improve health-related quality of life among older adults discharged from
Purpose: To evaluate a multifaceted transitional care intervention including home-based exercise strategies for at-risk older people on functional status, independence in activities of daily living (ADLs) and walking ability.

Study Design: 3 . Quality Rating: 3. $\mathrm{N}=128$

Setting: 1 .

Country: 4.

Medical Diagnosis; 0.

Age: 65+

\section{Purpose:}

To test the hypothesis that the extent to which family caregivers of older people

discharged home from hospital felt prepared to provide care at home would be positively influenced by their inclusion in the new Further Enabling Care at Home program.

Study Design: 1 . Quality Rating: 2. Global Rating (for weak/moderate articles): 1 .

$\mathrm{N}=175$

Setting: 1 .

Country: 4 .

Medical Diagnosis; 1.

Age: $70+$

Purpose: To examine if a low-cost video-based exercise program can improve outcomes for seniors transitioning from acute care to home.

Study Design: 1 . Quality Rating: 3. $\mathrm{N}=53$

Setting: 5

Country: 4

Medical Diagnosis; 0 Age: 65+ Intervention: 86
Total number of intervention groups:

Two

Number of participants allocated to each intervention group:

Intervention: 64

Control: 64

Outcomes and time points collected and reported:

Data was collected: baseline, 4, 12, 24 weeks.

Instrumental activities of daily living and activities of daily living (2)

Walking impairment (2)

Total number of intervention groups:

Two.

Number of participants allocated to each intervention group:

Control: 89

Outcomes and time points collected and reported:

Data collection times were as follows: Time 1, within four days of discharge; Time 2, 15-21 days after discharge; and Time 3, six weeks after discharge.

Primary outcome for caregivers: their preparedness to provide care at home for the patient (3).

\section{Total number of} intervention groups: Two.

Number of participants allocated to each intervention group: Intervention: 19

Control: 34

Outcomes and time points collected and reported:

Falls (2), health-related quality of life (3), participation in activities
Measurement Tools:

Berg Balance

Scale, the Timed Up

and Go Test, an

isometric muscle power test, and a walk test.

\section{Reliability/validity} reported? 1 .

\section{Measurement Tools:}

Component

Number: One.

Type: 2 .

Mode

Medium: 4 (face-to-

face, telephone

contact).

Format: 1 .

Treatment

Provider: 3 .

Dose

Amount: Unclear.

Frequency: within

48 hours of

discharge, weekly $x$

4 weeks, monthly for

5 months.

Duration: 24 weeks.

Heterogeneity: 2;3

Approach: 2.

Component

Number: two.

Type: 3 (Supportive

Caregiving Scale from

the Family Care

Inventory.

Symptom Assessment

Scale scores

The Family Appraisal

of Caregiving

Questionnaire -

Palliative Care

SF-12v2 Health

Survey

Barthel Activities of

Daily Living Index

Connor-Davidson

Resilience Scale

Reliability/validity reported? 1 .

Measurement Tools:

The utility and visual analogue scale components of the EQ-5D instrument Frenchay Activities Index

The Balance Outcome Measure for Elder

Rehabilitation

(BOOMER), the 15second timed sit-tostand test, and the 2-minute walk test care and education).

Mode

Medium: 2.

Format: 1 .

Treatment

Provider: 1.

Dose

Amount: Not

reported.

Frequency: one-

week post discharge,

7-10 days postdischarge, 14 days post-discharge.

Duration: 2-weeks. Heterogeneity: 1;4.

Approach: 1.

Component

Number: 2 .

Type:

3(education/supporti ve care).

Mode

Medium: 4 (written, face-to-face, telephone contact).

Indicator of quality used: 1.

Study Findings:

Outcome \#1: 2. Statistically Significant: Intervention: 1 IADL scores (P<0.001) ADL scores $(\mathrm{P}<$ 0.001) WIQ scale scores $(\mathrm{P}<0.001)$

Control: 00 .

Outcome \#2: 2.

Statistical

significance:

Intervention: 00 .

Control: 00.

Indicator of quality used: 1 .

Study Findings:

Outcome \#1: 3

Statistical

significance:

Intervention: 1

Preparedness for

stress of caregiving $\mathrm{p}=0.004$

To get support from

system $\mathrm{p}=0.012$ 


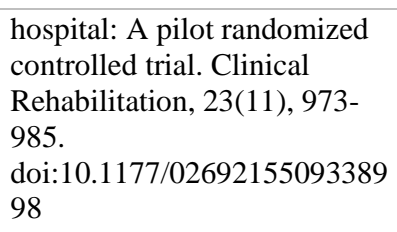

Date of Study: 2017 of daily living (2), physical capacity (2) and

fear of falling (3).

Face-to-face follow-up at

2 months and telephone

follow-up at six months

post-discharge.
The Activities specific $\underline{\text { Dose }}$ Balance Confidence (ABC) Scale.

Reliability/validity reported? 1.
Amount: Not

reported.

Frequency: 1/week.

Duration: $<8$ weeks.

Heterogeneity: 1;3.

Approach: 1. 
Appendix G: Description of Included Studies

\begin{tabular}{|l|l|l|l|}
\hline Study Characteristics (38 Studies) & Frequency & Percent \\
\hline Quality of study & Strong & 17 & $36.9 \%$ \\
\cline { 2 - 4 } & Moderate & 12 & $26 . \%$ \\
\cline { 2 - 4 } & Weak & 9 & $19.5 \%$ \\
\hline \multirow{4}{*}{ Country of origin } & Europe & 17 & $36.9 \%$ \\
\cline { 2 - 4 } & Australia & 8 & $17.4 \%$ \\
\cline { 2 - 4 } & The United States & 6 & $13 \%$ \\
\cline { 2 - 4 } & Asia & 7 & $15.2 \%$ \\
\hline Population & Medical Unit & 11 & $23.9 \%$ \\
\cline { 2 - 4 } & Geriatric Unit & 5 & $10.8 \%$ \\
\cline { 2 - 4 } & Medical/Surgical & 3 & $6.5 \%$ \\
\cline { 2 - 4 } & Not Specified & 27 & $58.7 \%$ \\
\hline Medical Diagnosis & Cardiac Stroke & 6 & $13 \%$ \\
\cline { 2 - 4 } & Nutritional Health Risk & 4 & $8.7 \%$ \\
\cline { 2 - 4 } & Stroke & 1 & $2.2 \%$ \\
\cline { 2 - 4 } & Hip Fracture & 1 & $2.2 \%$ \\
\cline { 2 - 4 } & Not Specified & 34 & $86.9 \%$ \\
\hline \multirow{4}{*}{$\begin{array}{l}\text { Number of } \\
\text { experimental groups }\end{array}$} & One & 40 & $4.3 \%$ \\
\cline { 2 - 4 } & Two & 2 & \\
\cline { 2 - 4 } & Three & & \\
\hline
\end{tabular}

\section{Appendix H: Description of Intervention Characteristics}

\begin{tabular}{|l|l|l|l|}
\hline Intervention Characteristics (46 Interventions) & Frequency & Percent \\
\hline Component: Type & Educational & 3 & $6.5 \%$ \\
\cline { 2 - 4 } & Supportive Care & 7 & $15.2 \%$ \\
\cline { 2 - 4 } & Medication Management & 1 & $2.2 \%$ \\
\cline { 2 - 4 } & Organizational & 0 & $0 \%$ \\
\cline { 2 - 4 } & $\begin{array}{l}\text { Supportive Care + } \\
\text { Organizational }\end{array}$ & 14 & $30.4 \%$ \\
\cline { 2 - 4 } & $\begin{array}{l}\text { Educational + Supportive } \\
\text { Care }\end{array}$ & 12 & $26 \%$ \\
\cline { 2 - 4 } & $\begin{array}{l}\text { Supportive Care + } \\
\text { Medication Management }\end{array}$ & 3 & $6.5 \%$ \\
\cline { 2 - 4 } & $\begin{array}{l}\text { Educational + Supportive } \\
\text { Care + Medication } \\
\text { Management }\end{array}$ & 3 & $6.5 \%$ \\
\cline { 2 - 4 } & $\begin{array}{l}\text { Educational + Supportive } \\
\text { Care + Medication } \\
\text { Management + } \\
\text { Organizational }\end{array}$ & 2 & $4.3 \%$ \\
\cline { 2 - 4 } & $\begin{array}{l}\text { Supportive Care + } \\
\text { Medication Management + } \\
\text { Organizational }\end{array}$ & 1 & $23.9 \%$ \\
\hline Component: Number & One & 11 & $63 \%$ \\
\cline { 2 - 4 } & Two & 29 & $8.7 \%$ \\
\cline { 2 - 4 } & Three & \\
\hline
\end{tabular}




\begin{tabular}{|c|c|c|c|}
\hline & Four & 2 & $4.3 \%$ \\
\hline \multirow[t]{7}{*}{ Mode: Medium } & Face-to-Face & 16 & $34.8 \%$ \\
\hline & Telephone Call & 6 & $13 \%$ \\
\hline & Written & 1 & $2.2 \%$ \\
\hline & $\begin{array}{l}\text { Face-to-Face + Phone } \\
\text { Contact }\end{array}$ & 12 & $26.1 \%$ \\
\hline & $\begin{array}{l}\text { Face-to-Face + Phone } \\
\text { Contact + Written }\end{array}$ & 8 & $17.3 \%$ \\
\hline & Face-to-Face + Written & 2 & $4.3 \%$ \\
\hline & Phone Contact + Written & 1 & $2.2 \%$ \\
\hline \multirow{3}{*}{$\begin{array}{l}\text { Mode: Treatment } \\
\text { Provider }\end{array}$} & Nurse & 11 & $23.9 \%$ \\
\hline & Multidisciplinary & 17 & $37 \%$ \\
\hline & Other & 18 & $39.1 \%$ \\
\hline \multirow[t]{3}{*}{ Mode: Format } & One-to-One & 28 & $60.9 \%$ \\
\hline & Group & 17 & $37 \%$ \\
\hline & Combination & 1 & $2.2 \%$ \\
\hline \multirow[t]{6}{*}{ Dose: Amount } & 15 minutes & 2 & $4.3 \%$ \\
\hline & 30 minutes & 2 & $4.3 \%$ \\
\hline & 45 minutes & 2 & $4.3 \%$ \\
\hline & 60 minutes & 4 & $8.7 \%$ \\
\hline & 120 minutes & 3 & $6.5 \%$ \\
\hline & Not Reported & 33 & $71 \%$ \\
\hline \multirow[t]{7}{*}{ Dose: Frequency } & Once/month & 7 & $15 \%$ \\
\hline & Twice/month & 4 & $8.7 \%$ \\
\hline & Three times/month & 4 & $8.7 \%$ \\
\hline & Four times/month & 7 & $15.2 \%$ \\
\hline & Five times/month & 2 & $4.3 \%$ \\
\hline & Variable & 19 & $41.3 \%$ \\
\hline & Not Reported & 3 & $6.5 \%$ \\
\hline \multirow{3}{*}{$\begin{array}{l}\text { Dose Heterogeneity } \\
\text { (Fixed/Variable) }\end{array}$} & Fixed & 33 & $71.7 \%$ \\
\hline & Variable & 8 & $17.4 \%$ \\
\hline & Not Reported & 5 & $10.9 \%$ \\
\hline \multirow[t]{3}{*}{ Dose Heterogeneity } & Tailored & 13 & $28.3 \%$ \\
\hline & Untailored & 28 & $60.9 \%$ \\
\hline & Not Reported & 5 & $10.9 \%$ \\
\hline \multirow[t]{4}{*}{ Duration } & One-Three Months & 32 & $69.6 \%$ \\
\hline & Four-Six Months & 5 & $10.9 \%$ \\
\hline & Seven-Twelve Months & 6 & $13 \%$ \\
\hline & Not Reported & 3 & $6.5 \%$ \\
\hline \multirow[t]{3}{*}{ Approach } & Standardized & 21 & $45.7 \%$ \\
\hline & Tailored & 8 & $17.4 \%$ \\
\hline & Combination & 17 & $37 \%$ \\
\hline
\end{tabular}


Appendix I: Component Types

\begin{tabular}{|c|c|c|}
\hline Component type & Operational Definition & $\begin{array}{c}\text { Examples from Included } \\
\text { Interventions } \\
\end{array}$ \\
\hline Educational & $\begin{array}{l}\text { Any intervention which } \\
\text { includes information, } \\
\text { resources, and/or an } \\
\text { education aspect. }\end{array}$ & $\begin{array}{l}\text { Falls prevention strategies, } \\
\text { nutrition-based counselling, } \\
\text { written/video discharge } \\
\text { instructions, patient-teaching. }\end{array}$ \\
\hline Supportive Care & $\begin{array}{l}\text { Any social, physical, and } \\
\text { emotional support and/or } \\
\text { resources for the client, } \\
\text { family, and/or caregiver } \\
\text { throughout the transition from } \\
\text { acute care to home. }\end{array}$ & $\begin{array}{l}\text { Discharge follow-up in the } \\
\text { community, assessment of } \\
\text { health status post-discharge, } \\
\text { enlisting community } \\
\text { supports, coordinating care, } \\
\text { and continuation of care in } \\
\text { the community. }\end{array}$ \\
\hline Organizational & $\begin{array}{l}\text { Any intervention which } \\
\text { addresses healthcare provider } \\
\text { activities and/or which } \\
\text { support the provision of } \\
\text { effective, efficient, equitable, } \\
\text { timely, safe and patient- } \\
\text { centered transitional care. }\end{array}$ & $\begin{array}{l}\text { Rehabilitation programs, } \\
\text { discharge planning } \\
\text { summaries, early discharge } \\
\text { planning and } \\
\text { multidisciplinary } \\
\text { coordination }\end{array}$ \\
\hline Medication Management & $\begin{array}{l}\text { Any intervention that } \\
\text { provides medication } \\
\text { reconciliation, verification, } \\
\text { and/or clarification. }\end{array}$ & $\begin{array}{l}\text { Medication counselling, } \\
\text { reconciliation, identification } \\
\text { of potential drug related } \\
\text { problems, and the transfer of } \\
\text { the patient's medication } \\
\text { review from inpatient to } \\
\text { outpatient providers }\end{array}$ \\
\hline
\end{tabular}

Appendix J: Outcomes of Quality of Care

\begin{tabular}{|l|l|l|l|}
\hline Quality of Care Outcomes (46 Interventions) & Frequency & Percent \\
\hline \multirow{3}{*}{$\begin{array}{l}\text { Health Service } \\
\text { utilization outcomes } \\
(\mathrm{n}=23)\end{array}$} & Readmission Rates & 22 & $47.8 \%$ \\
\cline { 2 - 4 } & $\begin{array}{l}\text { Emergency Department } \\
\text { Visits }\end{array}$ & 3 & $6.5 \%$ \\
\cline { 2 - 4 } & Primary Care Visits & 3 & $6.5 \%$ \\
\hline \multirow{4}{*}{$\begin{array}{l}\text { Health Status } \\
\text { outcomes (n=23) }\end{array}$} & Functional/Physical Status & 14 & $30.4 \%$ \\
\cline { 2 - 4 } & Activities of Daily Living & 6 & $13 \%$ \\
\cline { 2 - 4 } & Falls & 3 & $6.5 \%$ \\
\cline { 2 - 4 } & Mortality Rates & 3 & $6.5 \%$ \\
\cline { 2 - 4 } & Drug-Related Problems & 2 & $4.3 \%$ \\
\cline { 2 - 4 } & Nutritional Status & 1 & $2.2 \%$ \\
\cline { 2 - 4 } & Frailty & 1 & $2.2 \%$ \\
\hline \multirow{4}{*}{$\begin{array}{l}\text { Self-Rated Outcomes } \\
(\mathrm{n}=13)\end{array}$} & Quality of Life & $6.3 \%$ \\
\cline { 2 - 4 } & Self-Efficacy Change & 2 & $2.2 \%$ \\
\cline { 2 - 4 } & Self-Rated Symptoms & 1 & \\
\hline
\end{tabular}




\begin{tabular}{|l|l|l|l|}
\hline & $\begin{array}{l}\text { Experiences of } \\
\text { Safety/Security }\end{array}$ & 2 & $4.3 \%$ \\
\cline { 2 - 4 } & Life Satisfaction & 1 & $2.2 \%$ \\
\cline { 2 - 4 } & Quality of Care & 1 & $2.2 \%$ \\
\cline { 2 - 4 } & Self-Rated Health & 1 & $2.2 \%$ \\
\cline { 2 - 4 } & Caregiver Preparedness & 1 & $2.2 \%$ \\
\cline { 2 - 4 } & Patient/Caregiver Stress & 1 & $2.2 \%$ \\
\hline
\end{tabular}

Appendix K: Combinations of Outcomes (Health-Service, Health Status, Self-Rated)

\begin{tabular}{|c|c|c|}
\hline Combinations & Frequency (Out of 46) & Percent (Out of 46) \\
\hline $\begin{array}{c}\text { Health-Service Utilization } \\
\text { Outcomes + Health- Status } \\
\text { Outcomes }\end{array}$ & 4 & $8.6 \%$ \\
\hline $\begin{array}{c}\text { Health- Service Utilization } \\
\text { Outcomes + Self-Rated } \\
\text { Outcomes }\end{array}$ & 1 & $2.1 \%$ \\
\hline $\begin{array}{c}\text { Self-Rated + Health- Status } \\
\text { Outcomes }\end{array}$ & 5 & $10.8 \%$ \\
\hline $\begin{array}{c}\text { Health-Service Utilization } \\
\text { Outcomes + Health Status } \\
\text { Outcomes + Self-Rated } \\
\text { Outcomes }\end{array}$ & 1 & $2.1 \%$ \\
\hline
\end{tabular}

\section{Appendix L: An Overview of Studies Yielding Statistical Significance Based on Intervention Characteristic}

\begin{tabular}{|l|l|l|l|l|}
\hline $\begin{array}{l}\text { Intervention } \\
\text { characteristic }\end{array}$ & $\begin{array}{l}\text { Sub-category } \\
\text { within the } \\
\text { Characteristic (n, } \\
\text { \%) }\end{array}$ & $\begin{array}{l}\text { \# of Studies with } \\
\text { SS in Self-Rated } \\
\text { Outcomes (\%) }\end{array}$ & $\begin{array}{l}\text { \# of Studies with } \\
\text { SS in Health Status } \\
\text { Outcomes (\%) }\end{array}$ & $\begin{array}{l}\text { \# of Studies with } \\
\text { SS in Health } \\
\text { Utilization } \\
\text { Outcomes (\%) }\end{array}$ \\
\hline $\begin{array}{l}\text { Component type } \\
(\mathrm{n}=46)\end{array}$ & Education (n=3) & $0(0 \%)$ & $1 / 3(33 \%)$ & $0(0 \%)$ \\
\cline { 2 - 5 } & $\begin{array}{l}\text { Medication } \\
\text { management (n=1, } \\
2.2 \%)\end{array}$ & $0 / 1(0 \%)$ & $0 / 1(0 \%)$ & $0(0 \%)$ \\
\cline { 2 - 5 } & $\begin{array}{l}\text { Supportive care (n= } \\
7,15.2 \%)\end{array}$ & $1 / 3(33 \%)$ & $1 / 6(16.5 \%)$ & $0 / 2(0 \%)$ \\
\cline { 2 - 5 } & $\begin{array}{l}\text { Combination (n= } \\
35,76.1 \%)\end{array}$ & $7 / 9(77.7 \%)$ & $3 / 13(23 \%)$ & $12 / 21(57 \%)$ \\
\hline \multirow{2}{*}{$\begin{array}{l}\text { Mode: Medium } \\
\text { (n=46) }\end{array}$} & $\begin{array}{l}\text { Face to face (n=16, } \\
34.8 \%)\end{array}$ & $2 / 3(66.6 \%)$ & $4 / 11(36.3 \%)$ & $2 / 7(28.5 \%)$ \\
\cline { 2 - 5 } & $\begin{array}{l}\text { Written (n=1, } \\
2.2 \%)\end{array}$ & 0 & 0 & $0 / 1(0 \%)$ \\
\cline { 2 - 5 } & $\begin{array}{l}\text { Telephone Contact } \\
(\mathrm{n}=6,13 \%)\end{array}$ & $1 / 2(50 \%)$ & $0 / 2(0 \%)$ & $1 / 4(25 \%)$ \\
\cline { 2 - 5 } & $\begin{array}{l}\text { Combination (n= } \\
23,50 \%)\end{array}$ & $5 / 8(62.5 \%)$ & $1 / 10(10 \%)$ & $9 / 11(81.5 \%)$ \\
\hline
\end{tabular}




\begin{tabular}{|c|c|c|c|c|}
\hline \multirow[t]{3}{*}{$\begin{array}{l}\text { Mode: Format } \\
(\mathrm{n}=46)\end{array}$} & $\begin{array}{l}\text { One-to-one }(\mathrm{n}=28, \\
60.9 \%)\end{array}$ & $2 / 5(40 \%)$ & $4 / 16(25 \%)$ & $8 / 15(53.3)$ \\
\hline & Group $(n=17,37 \%)$ & $5 / 7(71.4 \%)$ & $1 / 6(16 \%)$ & $4 / 8(50 \%)$ \\
\hline & $\begin{array}{l}\text { Combination }(\mathrm{n}=1 \text {, } \\
2.2 \%)\end{array}$ & $1 / 1(100 \%)$ & $0 / 1(0 \%)$ & 0 \\
\hline \multirow[t]{3}{*}{$\begin{array}{l}\text { Mode: Treatment } \\
\text { Provider }(n=46)\end{array}$} & $\begin{array}{l}\text { Nurse }(\mathrm{n}=11, \\
23.9 \%)\end{array}$ & $2 / 2(100 \%)$ & $0 / 3(0 \%)$ & $7 / 9(77.7 \%)$ \\
\hline & $\begin{array}{l}\text { Multidisciplinary } \\
(\mathrm{n}=17,37 \%)\end{array}$ & $6 / 7(85.7 \%)$ & $2 / 6(33.3 \%)$ & $4 / 8(50 \%)$ \\
\hline & $\begin{array}{l}\text { Other }(\mathrm{n}=18, \\
39.1 \%)\end{array}$ & $0 / 4(0 \%)$ & $3 / 14(21.4 \%)$ & $1 / 6(16.6 \%)$ \\
\hline \multirow[t]{2}{*}{$\begin{array}{l}\text { Dose Heterogeneity } \\
(\mathrm{n}=41)\end{array}$} & $\begin{array}{l}\text { Fixed }(n=33, \\
71.7 \%)\end{array}$ & $6 / 9(66.6 \%)$ & $4 / 14(28.5 \%)$ & $8 / 18(44.4 \%)$ \\
\hline & $\begin{array}{l}\text { Variable }(\mathrm{n}=8, \\
17.4 \%)\end{array}$ & $2 / 3(66.6 \%)$ & $1 / 4(25 \%)$ & $4 / 5(80 \%)$ \\
\hline \multirow[t]{2}{*}{$\begin{array}{l}\text { Dose Heterogeneity } \\
(\mathrm{n}=41)\end{array}$} & $\begin{array}{l}\text { Tailored }(n=13, \\
28.3)\end{array}$ & $4 / 7(57.1 \%)$ & $0 / 6(0 \%)$ & $4 / 6(66.6 \%)$ \\
\hline & $\begin{array}{l}\text { Untailored }(n=28, \\
60.9 \%)\end{array}$ & $4 / 5(80 \%)$ & $5 / 12(41.6 \%)$ & $8 / 17(47 \%)$ \\
\hline \multirow[t]{3}{*}{ Approach $(n=46)$} & $\begin{array}{l}\text { Standardized }(\mathrm{n}= \\
21,45.7 \%)\end{array}$ & $4 / 8(50 \%)$ & $2 / 11(18 \%)$ & $4 / 12(33.3 \%)$ \\
\hline & $\begin{array}{l}\text { Tailored }(n=8, \\
17.4 \%)\end{array}$ & 1/1 (100\%) & $1 / 5(20 \%)$ & $1 / 2(50 \%)$ \\
\hline & $\begin{array}{l}\text { Combination }(\mathrm{n}= \\
17,37 \%)\end{array}$ & $3 / 4(75 \%)$ & $2 / 7(28.5 \%)$ & $7 / 9(77.7 \%)$ \\
\hline
\end{tabular}

\section{Appendix M: An Overview of Studies Yielding Non-Significant Findings Based on Intervention Characteristic}

\begin{tabular}{|c|c|c|c|c|}
\hline $\begin{array}{l}\text { Intervention } \\
\text { characteristic }\end{array}$ & $\begin{array}{l}\text { Sub-category } \\
\text { within the } \\
\text { characteristic (n, } \\
\% \text { ) }\end{array}$ & $\begin{array}{l}\text { \# of studies with } \\
\text { NS in self-rated } \\
\text { outcomes }(\%)\end{array}$ & $\begin{array}{l}\text { \# of studies with } \\
\text { NS in Health } \\
\text { Status Outcomes } \\
(\%)\end{array}$ & $\begin{array}{l}\text { \# of studies with } \\
\text { NS in health } \\
\text { utilization } \\
\text { outcomes }(\%)\end{array}$ \\
\hline \multirow{4}{*}{$\begin{array}{l}\text { Component type } \\
(\mathrm{n}=46)\end{array}$} & Education $(\mathrm{n}=3)$ & $0(0 \%)$ & $2 / 3(66.6 \%)$ & $0(0 \%)$ \\
\hline & $\begin{array}{l}\text { Medication } \\
\text { management }(\mathrm{n}=1 \text {, } \\
2.2 \%)\end{array}$ & $1 / 1(100 \%)$ & $1 / 1(100 \%)$ & $0(0 \%)$ \\
\hline & $\begin{array}{l}\text { Supportive care }(\mathrm{n}= \\
7,15.2 \%)\end{array}$ & $2 / 3(66.6 \%)$ & $2 / 6(33.3 \%)$ & $1 / 2(50 \%)$ \\
\hline & $\begin{array}{l}\text { Combination }(\mathrm{n}= \\
35,76.1 \%)\end{array}$ & $2 / 9(22.2 \%)$ & $7 / 13(53.8 \%)$ & $9 / 21(42.8 \%)$ \\
\hline \multirow[t]{4}{*}{$\begin{array}{l}\text { Mode: Medium } \\
(\mathrm{n}=46)\end{array}$} & $\begin{array}{l}\text { Face to face }(\mathrm{n}=16, \\
34.8 \%)\end{array}$ & $1 / 3(33.3 \%)$ & $5 / 11(45.4 \%)$ & $5 / 7(71.4 \%)$ \\
\hline & $\begin{array}{l}\text { Written }(n=1, \\
2.2 \%)\end{array}$ & 0 & 0 & $1 / 1(100 \%)$ \\
\hline & $\begin{array}{l}\text { Telephone Contact } \\
(\mathrm{n}=6,13 \%)\end{array}$ & $1 / 2(50 \%)$ & $2 / 2(100 \%)$ & $2 / 4(50 \%)$ \\
\hline & $\begin{array}{l}\text { Combination }(\mathrm{n}= \\
23,50 \%)\end{array}$ & $3 / 8(37.5 \%)$ & $5 / 10(50 \%)$ & $2 / 11(18.1 \%)$ \\
\hline \multirow[t]{3}{*}{$\begin{array}{l}\text { Mode: Format } \\
(\mathrm{n}=46)\end{array}$} & $\begin{array}{l}\text { One-to-one }(\mathrm{n}=28, \\
60.9 \%)\end{array}$ & $3 / 5(60 \%)$ & $9 / 16(56.25 \%)$ & $7 / 15(46.6 \%)$ \\
\hline & Group $(n=17,37 \%)$ & $2 / 7(28.5 .4 \%)$ & $3 / 6(50 \%)$ & $3 / 8(37.5 \%)$ \\
\hline & $\begin{array}{l}\text { Combination }(\mathrm{n}=1, \\
2.2 \%)\end{array}$ & $0 / 1(0 \%)$ & $0 / 1(0 \%)$ & $0(0 \%)$ \\
\hline
\end{tabular}




\begin{tabular}{|c|c|c|c|c|}
\hline \multirow[t]{3}{*}{$\begin{array}{l}\text { Mode: Treatment } \\
\text { Provider }(n=46)\end{array}$} & $\begin{array}{l}\text { Nurse }(n=11, \\
23.9 \%)\end{array}$ & $0 / 2(0 \%)$ & $1 / 3(33.3 \%)$ & $2 / 9(22.2 \%)$ \\
\hline & $\begin{array}{l}\text { Multidisciplinary } \\
(\mathrm{n}=17,37 \%)\end{array}$ & $1 / 7(14.2 \%)$ & $1 / 6(16.6 \%)$ & $4 / 8(50 \%)$ \\
\hline & $\begin{array}{l}\text { Other }(\mathrm{n}=18, \\
39.1 \%)\end{array}$ & $4 / 4(100 \%)$ & $10 / 14(71.4 \%)$ & $4 / 6(66.6 \%)$ \\
\hline \multirow[t]{2}{*}{$\begin{array}{l}\text { Dose Heterogeneity } \\
(n=41)\end{array}$} & $\begin{array}{l}\text { Fixed }(n=33, \\
71.7 \%)\end{array}$ & $3 / 9(33.3 \%)$ & $7 / 14(50 \%)$ & $9 / 18(50 \%)$ \\
\hline & $\begin{array}{l}\text { Variable }(\mathrm{n}=8, \\
17.4 \%)\end{array}$ & $1 / 3(33.3 \%)$ & $0 / 4(0 \%)$ & $1 / 5(20 \%)$ \\
\hline \multirow[t]{2}{*}{$\begin{array}{l}\text { Dose Heterogeneity } \\
(\mathrm{n}=41)\end{array}$} & $\begin{array}{l}\text { Tailored }(n=13, \\
28.3)\end{array}$ & $3 / 7(42.8 \%)$ & $3 / 6(50 \%)$ & $1 / 6(16.6 \%)$ \\
\hline & $\begin{array}{l}\text { Untailored }(n=28, \\
60.9 \%)\end{array}$ & $1 / 5(20 \%)$ & $4 / 12(33.3 \%)$ & $9 / 17(52.9 \%)$ \\
\hline \multirow[t]{3}{*}{ Approach $(n=46)$} & $\begin{array}{l}\text { Standardized }(\mathrm{n}= \\
21,45.7 \%)\end{array}$ & $4 / 8(50 \%)$ & 7/11 (63.6\%) & $7 / 12(58.3 \%)$ \\
\hline & $\begin{array}{l}\text { Tailored }(n=8, \\
17.4 \%)\end{array}$ & $0 / 1(0 \%)$ & $3 / 5(60 \%)$ & $1 / 2(50 \%)$ \\
\hline & $\begin{array}{l}\text { Combination }(\mathrm{n}= \\
17,37 \%)\end{array}$ & $1 / 4(25 \%)$ & $2 / 7(28.5 \%)$ & $2 / 9(22.2 \%)$ \\
\hline
\end{tabular}

\section{Appendix N: An Overview of Studies Yielding Variable Findings Based on Intervention Characteristic}

\begin{tabular}{|c|c|c|c|}
\hline $\begin{array}{l}\text { Intervention } \\
\text { characteristic }\end{array}$ & $\begin{array}{l}\text { Sub-category } \\
\text { within the } \\
\text { characteristic (n, } \\
\%)\end{array}$ & $\begin{array}{l}\text { \# of studies with } \\
\text { Variable Findings } \\
\text { in Health Status } \\
\text { Outcomes }(\%)\end{array}$ & $\begin{array}{l}\text { \# of studies with } \\
\text { Variable Findings } \\
\text { in health } \\
\text { utilization } \\
\text { outcomes }(\%)\end{array}$ \\
\hline \multirow{4}{*}{$\begin{array}{l}\text { Component type } \\
(\mathrm{n}=46)\end{array}$} & Education $(n=3)$ & $0 / 3(0 \%)$ & $0(0 \%)$ \\
\hline & $\begin{array}{l}\text { Medication } \\
\text { management }(n=1 \text {, } \\
2.2 \%)\end{array}$ & $0 / 1(0 \%)$ & $0(0 \%)$ \\
\hline & $\begin{array}{l}\text { Supportive care }(\mathrm{n}= \\
7,15.2 \%)\end{array}$ & $3 / 6(50 \%)$ & $1 / 2(50 \%)$ \\
\hline & $\begin{array}{l}\text { Combination }(\mathrm{n}= \\
35,76.1 \%)\end{array}$ & $3 / 13(23 \%)$ & $1 / 21(4.7 \%)$ \\
\hline \multirow[t]{4}{*}{$\begin{array}{l}\text { Mode: Medium } \\
(\mathrm{n}=46)\end{array}$} & $\begin{array}{l}\text { Face to face }(n=16, \\
34.8 \%)\end{array}$ & $2 / 11(18.1 \%)$ & $0 / 7(0 \%)$ \\
\hline & $\begin{array}{l}\text { Written }(\mathrm{n}=1, \\
2.2 \%)\end{array}$ & 0 & $0 / 1(0 \%)$ \\
\hline & $\begin{array}{l}\text { Telephone Contact } \\
(\mathrm{n}=6,13 \%)\end{array}$ & $0 / 2(0 \%)$ & $1 / 4(25 \%)$ \\
\hline & $\begin{array}{l}\text { Combination }(\mathrm{n}= \\
23,50 \%)\end{array}$ & $4 / 10(40 \%)$ & $0 / 11(0 \%)$ \\
\hline \multirow[t]{3}{*}{$\begin{array}{l}\text { Mode: Format } \\
(n=46)\end{array}$} & $\begin{array}{l}\text { One-to-one }(\mathrm{n}=28, \\
60.9 \%)\end{array}$ & $3 / 16(18.7 \%)$ & $0 / 15(0 \%)$ \\
\hline & Group $(n=17,37 \%)$ & $2 / 6(33.3 \%)$ & $1 / 8(12.5 \%)$ \\
\hline & $\begin{array}{l}\text { Combination }(\mathrm{n}=1 \text {, } \\
2.2 \%)\end{array}$ & $1 / 1(100 \%)$ & 0 \\
\hline $\begin{array}{l}\text { Mode: Treatment } \\
\text { Provider }(\mathrm{n}=46)\end{array}$ & $\begin{array}{l}\text { Nurse }(\mathrm{n}=11, \\
23.9 \%)\end{array}$ & $2 / 3(66.6 \%)$ & $0 / 9(0 \%)$ \\
\hline
\end{tabular}




\begin{tabular}{|l|l|l|l|}
\hline \multirow{5}{*}{} & $\begin{array}{l}\text { Multidisciplinary } \\
(\mathrm{n}=17,37 \%)\end{array}$ & $3 / 6(50 \%)$ & $0 / 8(0 \%)$ \\
\cline { 2 - 4 } & $\begin{array}{l}\text { Other }(\mathrm{n}=18, \\
39.1 \%)\end{array}$ & $1 / 14(7.1 \%)$ & $1 / 6(16.6 \%)$ \\
\hline $\begin{array}{l}\text { Dose Heterogeneity } \\
(\mathrm{n}=41)\end{array}$ & $\begin{array}{l}\text { Fixed }(\mathrm{n}=33, \\
71.7 \%)\end{array}$ & $3 / 14(21.4 \%)$ & $1 / 18(5.5 \%)$ \\
\cline { 2 - 4 } & $\begin{array}{l}\text { Variable }(\mathrm{n}=8, \\
17.4 \%)\end{array}$ & $3 / 4(75 \%)$ & $0 / 5(0 \%)$ \\
\hline \multirow{2}{*}{$\begin{array}{l}\text { Dose Heterogeneity } \\
(\mathrm{n}=41)\end{array}$} & $\begin{array}{l}\text { Tailored }(\mathrm{n}=13, \\
28.3)\end{array}$ & $3 / 6(50 \%)$ & $1 / 6(16.6 \%)$ \\
\cline { 2 - 4 } & $\begin{array}{l}\text { Untailored }(\mathrm{n}=28, \\
60.9 \%)\end{array}$ & $3 / 12(25 \%)$ & $0 / 17(0 \%)$ \\
\hline Approach (n=46) & $\begin{array}{l}\text { Standardized }(\mathrm{n}= \\
21,45.7 \%)\end{array}$ & $2 / 11(18 \%)$ & $1 / 12(8.3 \%)$ \\
\cline { 2 - 4 } & $\begin{array}{l}\text { Tailored }(\mathrm{n}=8, \\
17.4 \%)\end{array}$ & $1 / 5(20 \%)$ & $0 / 2(0 \%)$ \\
\cline { 2 - 4 } & $\begin{array}{l}\text { Combination }(\mathrm{n}= \\
17,37 \%)\end{array}$ & $3 / 7(42.8 \%)$ & $0 / 9(0 \%)$ \\
\hline
\end{tabular}

Appendix O: A Breakdown of Intervention Characteristics That Used A Combination of Components $(n=35,76.1 \%)$

\begin{tabular}{|c|c|c|c|c|}
\hline $\begin{array}{l}\text { Sub-Categories of } \\
\text { Component Type }\end{array}$ & \multirow{2}{*}{$\begin{array}{l}\text { Specific } \\
\text { Combination } \\
\text { Used (n, \%) }\end{array}$} & \multirow{2}{*}{$\begin{array}{l}\text { Self-Rated } \\
\text { Outcomes } \\
\text { (\# Studies with } \\
\text { SS/Total that } \\
\text { Used This } \\
\text { Combination) } \\
\text { QOL }(1 / 2) \\
\text { ESS }(0 / 1) \\
\text { CP }(1 / 1)\end{array}$} & \multirow{2}{*}{$\begin{array}{l}\text { Functional } \\
\text { Status } \\
\text { Outcomes } \\
\\
\text { FPS (4/9) } \\
\text { MR }(0 / 1) \\
\text { FALL }(0 / 1) \\
\text { ADL }(0 / 1)\end{array}$} & \multirow{2}{*}{$\begin{array}{l}\text { Health Utilization Outcomes (\% with } \\
\text { SS) } \\
\\
\text { RR }(2 / 5)\end{array}$} \\
\hline $\begin{array}{l}\text { 1. Educational } \\
\text { 2. Supportive } \\
\text { Care }\end{array}$ & & & & \\
\hline \multirow[t]{5}{*}{$\begin{array}{l}\text { 3. } \text { Medication } \\
\text { Management } \\
\text { 4. Organizational }\end{array}$} & $\begin{array}{l}2+4 \\
(\mathrm{n}=14,40 \%)\end{array}$ & $\begin{array}{l}\text { QOL }(1 / 1) \\
\text { SRH }(0 / 1) \\
\text { ESS }(0 / 1) \\
\text { SRS }(0 / 1) \\
\text { LSAT }(1 / 1) \\
\text { SEC }(1 / 1) \\
\text { QOC }(1 / 1)\end{array}$ & $\begin{array}{l}\text { ADL }(0 / 1) \\
\text { FRA }(0 / 1) \\
\text { ADL }(1 / 1)\end{array}$ & $\begin{array}{l}\mathrm{RR}(7 / 8) \\
\operatorname{PCV}(1 / 1)\end{array}$ \\
\hline & $\begin{array}{l}2+3 \\
(\mathrm{n}=3,8.5 \%)\end{array}$ & & $\operatorname{DRP}(1 / 1)$ & $\begin{array}{l}\mathrm{RR}(0 / 2) \\
\operatorname{PCV}(0 / 1)\end{array}$ \\
\hline & $\begin{array}{l}1+2+3 \\
(\mathrm{n}=3,8.5 \%)\end{array}$ & & $\begin{array}{l}\text { MR }(0 / 1) \\
\text { FALL }(0 / 0)\end{array}$ & $\begin{array}{l}\operatorname{RR}(2 / 2) \\
\operatorname{EDV}(1 / 1)\end{array}$ \\
\hline & $\begin{array}{l}2+3+4 \\
(\mathrm{n}=1,2.8)\end{array}$ & & & $\mathrm{RR}(0 / 1)$ \\
\hline & $\begin{array}{l}1+2+3+4 \\
(\mathrm{n}=2,5.7)\end{array}$ & SEC (1/1) & & $\mathrm{RR}(1 / 2)$ \\
\hline
\end{tabular}




\section{Legend}

\begin{tabular}{|l|l|l|}
\hline Self-Rated Outcomes & Health Status Outcomes & $\begin{array}{l}\text { Health-Service } \\
\text { Utilization } \\
\text { Outcomes }\end{array}$ \\
\hline QOL- Quality of Life & FPS- Functional/physical & RR- Readmission \\
rEC- Self-efficacy change & status & EDV-Emergency \\
SRS- Self-rated symptoms & ADL- Activities of Daily & department visits \\
ESS- Experiences of & Living & PCV-Primary care \\
safety/security & FALL- Falls & visits \\
LSAT- Life satisfaction & MR- Mortality rates & \\
QOC- Quality of care & DRP- Drug-related problems & \\
SRH- Self-rated health & NS- Nutritional status & \\
CP- Caregiver preparedness & FRA- Frailty & \\
& & \\
\hline
\end{tabular}

\section{Appendix P: A breakdown of intervention characteristics with A combination of Modes}

\begin{tabular}{|c|c|c|c|c|c|}
\hline $\begin{array}{l}\text { Sub-Categories of } \\
\text { Mode Type }\end{array}$ & $\begin{array}{l}\text { Number of } \\
\text { Studies that } \\
\text { used A } \\
\text { Combination } \\
\text { Approach } \\
(\%)\end{array}$ & $\begin{array}{l}\text { Specific } \\
\text { Combination Used } \\
(\mathbf{n}, \%)\end{array}$ & $\begin{array}{l}\text { Self-Rated } \\
\text { Outcomes } \\
\text { (\# of Studies with } \\
\text { SS/Total that } \\
\text { Used This } \\
\text { Combination) }\end{array}$ & $\begin{array}{l}\text { Functional } \\
\text { Status } \\
\text { Outcomes }\end{array}$ & $\begin{array}{l}\text { Health } \\
\text { Utilization } \\
\text { Outcomes (\% } \\
\text { with SS) }\end{array}$ \\
\hline \multirow{4}{*}{$\begin{array}{l}\text { 1. Face-to-face } \\
\text { 2. Phone contact } \\
\text { 3. Written }\end{array}$} & \multirow[t]{4}{*}{$\mathrm{N}=23,50 \%$} & $1+2(\mathrm{n}=12,52 \%)$ & $\begin{array}{l}\text { SRH }(0 / 1) \\
\text { LSAT }(1 / 1) \\
\text { QOC }(1 / 1)\end{array}$ & $\begin{array}{l}\text { ADL (1/2) } \\
\text { FPS (1/3) } \\
\text { MR (0/1) }\end{array}$ & $\mathrm{RR}(4 / 6)$ \\
\hline & & $1+3(n=2,8.6 \%)$ & QOL $(0 / 1)$ & FPS (1/1) & \\
\hline & & $2+3(n=1,4.3 \%)$ & & FALL $(0 / 1)$ & \\
\hline & & $\begin{array}{l}1+2+3(n=8, \\
34.7 \%)\end{array}$ & $\begin{array}{l}\text { QOL (3/4) } \\
\text { ESS (0/1) }\end{array}$ & $\begin{array}{l}\text { FPS }(2 / 5) \\
\text { FALL }(0 / 1) \\
\text { ADL }(0 / 1)\end{array}$ & $\begin{array}{l}\mathrm{RR}(5 / 5) \\
\operatorname{PCV}(1 / 1)\end{array}$ \\
\hline
\end{tabular}

\section{Legend}

\begin{tabular}{|l|l|l|}
\hline Self-Rated Outcomes & Health-Status Outcomes & $\begin{array}{l}\text { Health-Service } \\
\text { Utilization } \\
\text { Outcomes }\end{array}$ \\
\hline QOL- Quality of Life & FPS- Functional/physical & RR- Readmission \\
SEC- Self-efficacy change & status & EDV-Emergency \\
SRS- Self-rated symptoms & ADL- Activities of Daily & EDVartment visits \\
ESS- Experiences of & Living & PCV-Primary care \\
safety/security & FALL- Falls & visits \\
LSAT- Life satisfaction & MR- Mortality rates \\
QOC- Quality of care & DRP- Drug-related problems & \\
SRH- Self-rated health & NS- Nutritional status & \\
CP- Caregiver preparedness & FRA- Frailty & \\
& & \\
\hline
\end{tabular}




\section{Appendix Q: Chi-Square Results}

Health Service Utilization: The Type of Components the Intervention Comprised Of

\begin{tabular}{|c|c|c|c|c|}
\hline $\begin{array}{l}\text { The Type of Component(s) } \\
\text { Within the Intervention }\end{array}$ & Counts & $\begin{array}{l}\text { Non- } \\
\text { Significant }\end{array}$ & Significant & Total \\
\hline \multirow[t]{3}{*}{ Supportive Care } & Count & 1 & 0 & 1 \\
\hline & Expected Count & 0.5 & 0.5 & 1.0 \\
\hline & $\%$ Of Total & $4.5 \%$ & $0.00 \%$ & $4.5 \%$ \\
\hline \multirow[t]{3}{*}{ Combination } & Count & 9 & 12 & 21 \\
\hline & Expected Count & $9.5 \%$ & $11.5 \%$ & 21.0 \\
\hline & $\%$ Of Total & $40.9 \%$ & $54.5 \%$ & $95.5 \%$ \\
\hline \multirow[t]{3}{*}{ Total } & Count & 10 & 12 & 22 \\
\hline & Expected Count & 10 & & 22.0 \\
\hline & $\%$ of Total & $45.5 \%$ & $54.5 \%$ & $100 \%$ \\
\hline
\end{tabular}

Non-Significant Findings $\left(\chi_{(1)}^{2}=1.26, p=.262\right)$.

Health Status Outcomes: The Type of Components the Intervention Comprised Of

\begin{tabular}{|llrrr|}
\hline $\begin{array}{l}\text { The Type of Component(s) } \\
\text { within the Intervention }\end{array}$ & Counts & $\begin{array}{l}\text { Non- } \\
\text { Significant }\end{array}$ & Significant & Total \\
\hline Educational & Count & 2 & 1 & 3 \\
\hline & Expected Count & 2.1 & 0.9 & 3.0 \\
\hline & \% Of Total & $11.8 \%$ & $5.9 \%$ & $17.6 \%$ \\
\hline Supportive Care & Count & 2 & 1 & 3 \\
\hline & Expected Count & 2.1 & 0.9 & 3.0 \\
\hline & \% Of Total & $11.8 \%$ & $5.9 \%$ & $17.6 \%$ \\
\hline Medication Management & Count & 1 & 0 & 1 \\
\hline & Expected Count & 0.7 & 0.3 & 1 \\
\hline & \% Of Total & $5.9 \%$ & $0.00 \%$ & $5.9 \%$ \\
\hline Combination & Count & 7 & 3 & 10 \\
\hline & Expected Count & 7.1 & 2.9 & 10.0 \\
\hline & \% Of Total & $41.2 \%$ & $17.6 \%$ & $58.8 \%$ \\
\hline Total & Count & 12 & 5 & 17 \\
\hline & Expected Count & 12.0 & 5.0 & 17.0 \\
\hline & $\%$ of Total & $70.6 \%$ & $29.4 \%$ & $100 \%$ \\
\hline Non-Significant Findings $\left(\chi 2_{(3)}=\right.$ & 0.463 , p= .927), & & & \\
\hline
\end{tabular}


Self-Rated Outcomes: The Type of Components the Intervention Comprised Of

\begin{tabular}{|c|c|c|c|c|}
\hline $\begin{array}{l}\text { The Type of Component(s) } \\
\text { within the Intervention }\end{array}$ & Counts & $\begin{array}{l}\text { Non- } \\
\text { Significant }\end{array}$ & Significant & Total \\
\hline \multirow[t]{3}{*}{ Supportive Care } & Count & 2 & 1 & 3 \\
\hline & Expected Count & 1.2 & 1.8 & 3.0 \\
\hline & $\%$ Of Total & $15.4 \%$ & $7.7 \%$ & $23.1 \%$ \\
\hline \multirow[t]{3}{*}{ Medication Management } & Count & 1 & 0 & 1 \\
\hline & Expected Count & 0.4 & 0.6 & 1.0 \\
\hline & $\%$ Of Total & $7.7 \%$ & $0.00 \%$ & $7.7 \%$ \\
\hline \multirow[t]{3}{*}{ Combination } & Count & 2 & 7 & 9 \\
\hline & Expected Count & 3.5 & 5.5 & 9.0 \\
\hline & $\%$ Of Total & $15.4 \%$ & $53.8 \%$ & $69.2 \%$ \\
\hline \multirow[t]{3}{*}{ Total } & Count & 5 & 8 & 13 \\
\hline & Expected Count & 5.0 & 8.0 & 13.0 \\
\hline & $\%$ of Total & $38.5 \%$ & $61.5 \%$ & $100.0 .0 \%$ \\
\hline
\end{tabular}

Health Service Utilization: The Medium of the Intervention

\begin{tabular}{|c|c|c|c|c|}
\hline $\begin{array}{l}\text { How the Intervention was } \\
\text { Carried Out (Medium of } \\
\text { Intervention) }\end{array}$ & Counts & $\begin{array}{l}\text { Non- } \\
\text { Significant }\end{array}$ & Significant & Total \\
\hline \multirow[t]{3}{*}{ Face-to-Face } & Count & 5 & 2 & 7 \\
\hline & $\begin{array}{l}\text { Expected } \\
\text { Count }\end{array}$ & 3.2 & 3.8 & 7 \\
\hline & $\begin{array}{l}\% \text { Of } \\
\text { Total }\end{array}$ & $22.7 \%$ & $9.1 \%$ & $31.8 \%$ \\
\hline \multirow[t]{3}{*}{ Phone Contact } & Count & 2 & 1 & 3 \\
\hline & $\begin{array}{l}\text { Expected } \\
\text { Count }\end{array}$ & 1.4 & 1.6 & 3.0 \\
\hline & $\begin{array}{l}\% \text { Of } \\
\text { Total }\end{array}$ & $9.1 \%$ & $4.5 \%$ & $13.6 \%$ \\
\hline \multirow[t]{3}{*}{ Written } & Count & 1 & 0 & 1 \\
\hline & $\begin{array}{l}\text { Expected } \\
\text { Count }\end{array}$ & 0.5 & 0.5 & 1.0 \\
\hline & $\begin{array}{l}\% \text { Of } \\
\text { Total }\end{array}$ & $4.5 \%$ & $0.00 \%$ & $4.5 \%$ \\
\hline \multirow{3}{*}{ Combination } & Count & 2 & 9 & 11 \\
\hline & $\begin{array}{l}\text { Expected } \\
\text { Count }\end{array}$ & 5 & 6 & 11.0 \\
\hline & $\begin{array}{l}\% \text { Of } \\
\text { Total }\end{array}$ & $9.1 \%$ & $40.9 \%$ & $50 \%$ \\
\hline \multirow[t]{3}{*}{ Total } & Count & 10 & 12 & 22 \\
\hline & $\begin{array}{l}\text { Expected } \\
\text { Count }\end{array}$ & 10.0 & 12.0 & 22.0 \\
\hline & $\begin{array}{l}\% \text { of } \\
\text { Total }\end{array}$ & $45.5 \%$ & $54.5 \%$ & $100.0 \%$ \\
\hline
\end{tabular}

Non-Significant Findings $\left(\chi_{(3)}=6.949, p=0.074\right)$, 
Health Status Outcomes: The Medium of the Intervention

\begin{tabular}{|llrrr|}
\hline $\begin{array}{l}\text { How the Intervention was } \\
\text { Carried Out (Medium of } \\
\text { Intervention) }\end{array}$ & Counts & $\begin{array}{l}\text { Non- } \\
\text { Significant }\end{array}$ & Significant & Total \\
\hline Face-to-Face & Count & 5 & 4 & 9 \\
\hline & $\begin{array}{l}\text { Expected } \\
\text { Count }\end{array}$ & 6.4 & 2.6 & 9.0 \\
\hline & $\%$ Of Total & $29.4 \%$ & $23.5 \%$ & $52.9 \%$ \\
\hline Phone Contact & Count & 2 & 0 & 2 \\
\hline & $\begin{array}{l}\text { Expected } \\
\text { Count }\end{array}$ & 1.4 & .6 & 2.0 \\
\hline \% Of Total & $11.8 \%$ & $0 \%$ & $11.8 \%$ \\
\hline Combination & Count & 5 & 1 & 6 \\
\hline & Expected & 4.2 & 1.8 & 6.0 \\
\hline & Count & $29.4 \%$ & $5.9 \%$ & $35.3 \%$ \\
\hline Total & Count Total & 12 & 5 & 17 \\
\hline & $\begin{array}{l}\text { Expected } \\
\text { Count }\end{array}$ & 12.0 & 5.0 & 17.0 \\
\hline & $\%$ Of Total & $70.6 \%$ & $29.4 \%$ & $100.0 \%$ \\
\hline
\end{tabular}

Non-Significant Findings $\left(\chi 2_{(2)}=2.282, p=0.319\right)$,

\section{Self-Rated Outcomes: The Medium of Interventions}

\begin{tabular}{|c|c|c|c|c|}
\hline $\begin{array}{l}\text { How the Intervention was } \\
\text { Carried Out (Medium of } \\
\text { Intervention) }\end{array}$ & Counts & $\begin{array}{l}\text { Non- } \\
\text { Significant }\end{array}$ & Significant & Total \\
\hline \multirow[t]{3}{*}{ Face-to-Face } & Count & 1 & 2 & 3 \\
\hline & $\begin{array}{l}\text { Expected } \\
\text { Count }\end{array}$ & 1.2 & 1.8 & 3.0 \\
\hline & $\%$ Of Total & $7.7 \%$ & $15.4 \%$ & $23.1 \%$ \\
\hline \multirow[t]{3}{*}{ Phone Contact } & Count & 1 & 1 & 2 \\
\hline & $\begin{array}{l}\text { Expected } \\
\text { Count }\end{array}$ & .8 & 1.2 & 2.0 \\
\hline & $\%$ Of Total & $7.7 \%$ & $7.7 \%$ & $15.4 \%$ \\
\hline \multirow[t]{3}{*}{ Combination } & Count & 3 & 5 & 8 \\
\hline & $\begin{array}{l}\text { Expected } \\
\text { Count }\end{array}$ & 3.1 & 4.9 & 8.0 \\
\hline & $\%$ Of Total & $23.1 \%$ & $38.5 \%$ & $61.5 \%$ \\
\hline \multirow[t]{3}{*}{ Total } & Count & 5 & 8 & 13 \\
\hline & $\begin{array}{l}\text { Expected } \\
\text { Count }\end{array}$ & 5.0 & 8.0 & 13.0 \\
\hline & $\%$ of Total & $38.5 \%$ & $61.5 \%$ & $100.0 \%$ \\
\hline
\end{tabular}




\section{The Format of Interventions}

\begin{tabular}{|c|c|c|c|c|c|}
\hline Outcome & $\begin{array}{l}\text { How the Intervention } \\
\text { was Delivered } \\
\text { (Format of } \\
\text { Intervention) }\end{array}$ & Counts & $\begin{array}{l}\text { Non- } \\
\text { Significant }\end{array}$ & Significant & Total \\
\hline \multirow[t]{9}{*}{$\begin{array}{l}\text { Health-Service } \\
\text { Utilization }\end{array}$} & One-To-One & Count & 7 & 8 & 15 \\
\hline & & Expected Count & 6.8 & 8.2 & 15.0 \\
\hline & & $\%$ Of Total & $31.8 \%$ & $36.4 \%$ & $68.2 \%$ \\
\hline & Group & Count & 3 & 4 & 7 \\
\hline & & Expected Count & 3.2 & 3.8 & 7.0 \\
\hline & & $\%$ Of Total & $13.6 \%$ & $18.2 \%$ & $31.8 \%$ \\
\hline & Total & Count & 10 & 12 & 22 \\
\hline & & Expected Count & 10.0 & 12.0 & 22 \\
\hline & & $\%$ Of Total & $45.5 \%$ & $54.5 \%$ & $100.0 \%$ \\
\hline \multirow[t]{9}{*}{$\begin{array}{l}\text { Health Status } \\
\text { Outcomes }\end{array}$} & One-To-One & Count & 9 & 4 & 13 \\
\hline & & Expected Count & 2.8 & 1.2 & 4.0 \\
\hline & & $\%$ Of Total & $17.6 \%$ & $5.9 \%$ & $23.5 \%$ \\
\hline & Group & Count & 3 & 1 & 4 \\
\hline & & Expected Count & 2.8 & 1.2 & 4.0 \\
\hline & & $\%$ Of Total & $17.6 \%$ & $5.9 \%$ & $23.5 \%$ \\
\hline & Total & Count & 12 & 5 & 17 \\
\hline & & Expected Count & 12.0 & 5.0 & 17.0 \\
\hline & & $\%$ Of Total & $70.6 \%$ & $29.4 \%$ & $100.0 \%$ \\
\hline \multirow[t]{12}{*}{$\begin{array}{l}\text { Self-Rated } \\
\text { Outcomes }\end{array}$} & One-To-One & Count & 3 & 2 & 5 \\
\hline & & Expected Count & 1.9 & 3.1 & 5.0 \\
\hline & & $\%$ Of Total & $23.1 \%$ & $15.4 \%$ & $38.5 \%$ \\
\hline & Group & Count & 2 & 5 & 7 \\
\hline & & Expected Count & 2.7 & 4.3 & 7.0 \\
\hline & & $\%$ Of Total & $15.4 \%$ & $38.5 \%$ & $53.8 \%$ \\
\hline & Combination & Count & 0 & 1 & 1 \\
\hline & & Expected Count & 0.4 & 0.6 & 1.0 \\
\hline & & $\%$ Of Total & $0.00 \%$ & $2.20 \%$ & $\mathrm{n} / \mathrm{a}$ \\
\hline & Total & Count & 5 & 8 & 13 \\
\hline & & Expected Count & 5.0 & 8.0 & 13.0 \\
\hline & & $\%$ of Total & $38.5 \%$ & $61.5 \%$ & $100.0 \%$ \\
\hline
\end{tabular}

Non-Significant Findings Health Service Utilization: $\left[\chi 2_{(1)}=0.028, p=0.867\right]$.

Non-Significant Findings Health Status Outcomes: $\left[\chi_{(1)}=0.049, p=0.825\right]$.

Non-Significant Findings Self-Rated Outcomes: $\left[\chi_{(2)}=1.894, p=0.388\right]$. 
Mode: The Treatment Provider Who Implemented the Intervention

\begin{tabular}{|c|c|c|c|c|c|}
\hline Outcome & $\begin{array}{l}\text { Treatment } \\
\text { Provider }\end{array}$ & Counts & $\begin{array}{l}\text { Non- } \\
\text { Significant }\end{array}$ & Significant & Total \\
\hline \multirow[t]{12}{*}{ Health-Service Utilization } & Nurse & Count & 2 & 7 & 9 \\
\hline & & Expected Count & 4.1 & 4.9 & 9.0 \\
\hline & & $\%$ Of Total & $9.1 \%$ & $31.8 \%$ & $40.9 \%$ \\
\hline & Multidisciplinary & Count & 4 & 4 & 8 \\
\hline & & Expected Count & 3.6 & 4.4 & 8.0 \\
\hline & & $\%$ Of Total & $18.2 \%$ & $18.2 \%$ & $36.4 \%$ \\
\hline & Single-Discipline & Count & 4 & 1 & 5 \\
\hline & & Expected Count & 2.3 & 2.7 & 8.0 \\
\hline & & $\%$ Of Total & $18.2 \%$ & $4.5 \%$ & $22.7 \%$ \\
\hline & Total & Count & 10 & 12 & 22 \\
\hline & & Expected Count & 10.0 & 12.0 & 22.0 \\
\hline & & $\%$ of Total & $45.5 \%$ & $54.5 \%$ & $100.0 \%$ \\
\hline \multirow{12}{*}{ Health Status Outcomes } & Nurse & Count & 1 & 0 & 1 \\
\hline & & Expected Count & 0.7 & 0.3 & 1.0 \\
\hline & & $\%$ Of Total & $5.9 \%$ & $0.00 \%$ & $5.9 \%$ \\
\hline & Multidisciplinary & Count & 1 & 2 & 3 \\
\hline & & Expected Count & 2.1 & 0.9 & 3.0 \\
\hline & & $\%$ Of Total & $5.9 \%$ & $11.8 \%$ & $17.6 \%$ \\
\hline & Single-Discipline & Count & 10 & 3 & 13 \\
\hline & & Expected Count & 9.2 & 3.8 & 13.0 \\
\hline & & $\%$ Of Total & $58.8 \%$ & $29.4 \%$ & $76.5 \%$ \\
\hline & Total & Count & 12 & 5 & 17 \\
\hline & & Expected Count & 12.0 & 5.0 & 17 \\
\hline & & $\%$ of Total & $70.6 \%$ & $29.4 \%$ & $100.0 \%$ \\
\hline \multirow[t]{12}{*}{ Self-Rated Outcomes * } & Nurse & Count & 0 & 2 & 2 \\
\hline & & Expected Count & 0.8 & 1.2 & 2.0 \\
\hline & & $\%$ Of Total & $0.00 \%$ & $15.4 \%$ & $15.4 \%$ \\
\hline & Multidisciplinary & Count & 1 & 6 & 7 \\
\hline & & Expected Count & 2.7 & 4.3 & 7.0 \\
\hline & & $\%$ Of Total & $7.7 \%$ & $46.2 \%$ & $53.8 \%$ \\
\hline & Single-Discipline & Count & 4 & 0 & 4 \\
\hline & & Expected Count & 1.5 & 2.5 & 4.0 \\
\hline & & $\%$ Of Total & $30.8 \%$ & $0.00 \%$ & $30.8 \%$ \\
\hline & Total & Count & 5 & 8 & 13 \\
\hline & & Expected Count & 5.0 & 8.0 & 13.0 \\
\hline & & $\%$ of Total & $38.5 \%$ & $61.5 \%$ & $100.0 \%$ \\
\hline
\end{tabular}

Non-Significant Findings Health Service Utilization: $\left[\chi_{(2)}=4.433, p=0.109\right]$.

Non-Significant Findings Health Status Outcomes: $\left[\chi 2_{(2)}=2.674, p=0.263\right]$.

Significant Findings Self-Rated Outcomes: $\left[\chi 2_{(2)}=9.379, p=0.009\right]$. 
Self-Rated Outcomes: The Treatment Provider Who Implemented the Intervention

\begin{tabular}{|c|c|c|c|c|}
\hline $\begin{array}{l}\text { The Treatment } \\
\text { Provider Who } \\
\text { Implemented the } \\
\text { Intervention }\end{array}$ & Counts & $\begin{array}{l}\text { Non- } \\
\text { Significant }\end{array}$ & Significant & Total \\
\hline \multirow[t]{4}{*}{ Nurse } & Count & 0 & 2 & 2 \\
\hline & $\begin{array}{l}\text { Expected } \\
\text { Count }\end{array}$ & 0.8 & 1.2 & 2.0 \\
\hline & $\%$ Of Total & $0.0 \%$ & $15.4 \%$ & $15.4 \%$ \\
\hline & $\begin{array}{l}\text { Standardized } \\
\text { Residual }\end{array}$ & -0.9 & 0.7 & \\
\hline \multirow[t]{4}{*}{ Multidisciplinary } & Count & 1 & 6 & 7 \\
\hline & $\begin{array}{l}\text { Expected } \\
\text { Count }\end{array}$ & 2.7 & 4.3 & 7.0 \\
\hline & $\%$ Of Total & $7.7 \%$ & $46.2 \%$ & $53.8 \%$ \\
\hline & $\begin{array}{l}\text { Standardized } \\
\text { Residual }\end{array}$ & -1.0 & 0.8 & \\
\hline \multirow[t]{4}{*}{ Single-Discipline } & Count & 4 & 0 & 4 \\
\hline & $\begin{array}{l}\text { Expected } \\
\text { Count }\end{array}$ & 1.5 & 2.5 & 4.0 \\
\hline & $\%$ Of Total & $38.5 \%$ & $61.5 \%$ & $100.0 \%$ \\
\hline & $\begin{array}{l}\text { Standardized } \\
\text { Residual }\end{array}$ & $2.0 *$ & -1.6 & \\
\hline \multirow[t]{3}{*}{ Other } & Count & 5 & 8 & 13 \\
\hline & $\begin{array}{l}\text { Expected } \\
\text { Count }\end{array}$ & 5.0 & 8.0 & 13.0 \\
\hline & $\%$ Of Total & $38.5 \%$ & $61.5 \%$ & $100.0 \%$ \\
\hline
\end{tabular}

* Statistical Significance 
The Dose Heterogeneity Fixed/Variable of the Intervention

\begin{tabular}{|c|c|c|c|c|c|}
\hline Outcome & Fixed/Variable & Counts & $\begin{array}{l}\text { Non- } \\
\text { Significant }\end{array}$ & Significant & Total \\
\hline \multirow[t]{9}{*}{$\begin{array}{l}\text { Health-Service } \\
\text { Utilization }\end{array}$} & Fixed & Count & 9 & 8 & 17 \\
\hline & & Expected Count & 7.7 & 8.6 & 17.0 \\
\hline & & $\%$ Of Total & $40.9 \%$ & $36.4 \%$ & $77.3 \%$ \\
\hline & Variable & Count & 1 & 4 & 5 \\
\hline & & Expected Count & 2.3 & 2.7 & 5.0 \\
\hline & & $\%$ Of Total & $4.5 \%$ & $18.2 \%$ & $22.7 \%$ \\
\hline & Total & Count & 10 & 12 & 22 \\
\hline & & Expected Count & 10.0 & 12.0 & 22.0 \\
\hline & & $\%$ of Total & $45.5 \%$ & $54.5 \%$ & $100.0 \%$ \\
\hline \multirow[t]{9}{*}{$\begin{array}{l}\text { Health Status } \\
\text { Outcomes }\end{array}$} & Fixed & Count & 7 & 4 & 11 \\
\hline & & Expected Count & 6.4 & 4.6 & 11.0 \\
\hline & & $\%$ Of Total & $58.3 \%$ & $33.3 \%$ & $91.7 \%$ \\
\hline & Variable & Count & 0 & 1 & 1 \\
\hline & & Expected Count & 0.6 & 0.4 & 1.0 \\
\hline & & $\%$ Of Total & $0.00 \%$ & $8.3 \%$ & $8.3 \%$ \\
\hline & Total & Count & 7 & 5 & 12 \\
\hline & & Expected Count & 7.0 & 5.0 & 12 \\
\hline & & $\%$ of Total & $58 . \%$ & $41.7 \%$ & $100.0 \%$ \\
\hline \multirow[t]{9}{*}{ Self-Rated Outcomes } & Fixed & Count & 3 & 6 & 9 \\
\hline & & Expected Count & 3.0 & 6.0 & 9.0 \\
\hline & & $\%$ Of Total & $25.0 \%$ & $50.0 \%$ & $75.0 \%$ \\
\hline & Variable & Count & 1 & 2 & 3 \\
\hline & & Expected Count & 1.0 & 2.0 & 3.0 \\
\hline & & $\%$ Of Total & $8.3 \%$ & $16.7 \%$ & $25.0 \%$ \\
\hline & Total & Count & 4 & 8 & 12 \\
\hline & & Expected Count & 4.0 & 8.0 & 12.0 \\
\hline & & $\%$ of Total & $33.3 \%$ & $66.7 \%$ & $100.0 \%$ \\
\hline
\end{tabular}

Non-Significant Findings Health Service Utilization: $\left[\chi 2_{(1)}=1.691, p=0.193\right]$.

Non-Significant Findings Health Status Outcomes: [ $\left.\chi^{2}{ }_{(1)}=1.526, p=0.217\right]$.

Non-Significant Findings Self-Rated Outcomes: $\left[\chi_{(1)}=0.000, p=1.000\right]$. 
The Dose Heterogeneity Tailored/Untailored of the Intervention

\begin{tabular}{|c|c|c|c|c|c|}
\hline Outcome & $\begin{array}{l}\text { Tailored/Untailored } \\
\text { Intervention }\end{array}$ & Counts & $\begin{array}{l}\text { Non- } \\
\text { Significant }\end{array}$ & Significant & Total \\
\hline \multirow[t]{9}{*}{$\begin{array}{l}\text { Health-Service } \\
\text { Utilization }\end{array}$} & Tailored & Count & 1 & 4 & 5 \\
\hline & & Expected Count & 2.3 & 2.7 & 5.0 \\
\hline & & $\%$ Of Total & $4.5 \%$ & $18.2 \%$ & $22.7 \%$ \\
\hline & Untailored & Count & 9 & 8 & 17 \\
\hline & & Expected Count & 7.7 & 9.3 & 17.0 \\
\hline & & $\%$ Of Total & $40.9 \%$ & $36.4 \%$ & $77.3 \%$ \\
\hline & Total & Count & 10 & 12 & 22 \\
\hline & & Expect Count & 10.0 & 12.0 & 22.0 \\
\hline & & $\%$ of Total & $45.5 \%$ & $54.5 \%$ & $100.0 \%$ \\
\hline \multirow[t]{9}{*}{ Health Status Outcomes } & Tailored & Count & 3 & 0 & 3 \\
\hline & & Expected Count & 31.8 & 1.3 & 3.0 \\
\hline & & $\%$ Of Total & $25.0 \%$ & $0.0 \%$ & $25.0 \%$ \\
\hline & Untailored & Count & 4 & 5 & 9 \\
\hline & & Expected Count & 5.3 & 3.8 & 9.0 \\
\hline & & $\%$ Of Total & $33.3 \%$ & $41.7 \%$ & $75.0 \%$ \\
\hline & Total & Count & 7 & 5 & 12 \\
\hline & & Expected Count & 7.0 & 5.0 & 12.0 \\
\hline & & $\%$ Of Total & $58.3 \%$ & $41.7 \%$ & $100.0 \%$ \\
\hline \multirow[t]{9}{*}{ Self-Rated Outcomes } & Tailored & Count & 3 & 4 & 7 \\
\hline & & Expected Count & 2.3 & 4.7 & 7.0 \\
\hline & & $\%$ Of Total & $25.0 \%$ & $33.3 \%$ & $58.3 \%$ \\
\hline & Untailored & Count & 1 & 4 & 5 \\
\hline & & Expected Count & 1.7 & 3.3 & 5.0 \\
\hline & & $\%$ Of Total & $8.3 \%$ & $33.3 \%$ & $41.7 \%$ \\
\hline & Total & Count & 4 & 8 & 12.0 \\
\hline & & Expected Count & 4.0 & 8.0 & 12.0 \\
\hline & & $\%$ Of Total & $33.3 \%$ & $66.7 \%$ & $100.0 \%$ \\
\hline
\end{tabular}

Non-Significant Findings Health Service Utilization: $\left[\chi 2_{(1)}=1.691, p=0.193\right]$.

Non-Significant Findings Health Status Outcomes: $\left[\chi_{(1)}=2.857, p=0.091\right]$.

Non-Significant Findings Self-Rated Outcomes: [ $\left.\chi 2_{(2)}=0.686, p=0.408\right]$. 
The Approach of the Intervention: The Structure in Which the Intervention was Given

\begin{tabular}{|c|c|c|c|c|c|}
\hline Outcome & $\begin{array}{l}\text { Standardized/Tailored/ } \\
\text { Combination Intervention }\end{array}$ & Counts & $\begin{array}{c}\text { Non- } \\
\text { Significant }\end{array}$ & Significant & Total \\
\hline \multirow[t]{12}{*}{$\begin{array}{l}\text { Health-Service } \\
\text { Utilization }\end{array}$} & Standardized & Count & 7 & 4 & 11 \\
\hline & & $\begin{array}{l}\text { Expected } \\
\text { Count }\end{array}$ & 5.0 & 6.0 & 11.0 \\
\hline & & $\%$ Of Total & $31.8 \%$ & $18.2 \%$ & $50.0 \%$ \\
\hline & Tailored & Count & 1 & 1 & 2 \\
\hline & & $\begin{array}{l}\text { Expected } \\
\text { Count }\end{array}$ & 0.9 & 1.1 & 2.0 \\
\hline & & $\%$ Of Total & $4.5 \%$ & $4.5 \%$ & $9.1 \%$ \\
\hline & Combination & Count & 2 & 7 & 9 \\
\hline & & $\begin{array}{l}\text { Expected } \\
\text { Count }\end{array}$ & 4.1 & 4.9 & 9.0 \\
\hline & & $\%$ Of Total & $9.1 \%$ & $31.8 \%$ & $40.9 \%$ \\
\hline & Total & Count & 10 & 12 & 22 \\
\hline & & $\begin{array}{l}\text { Expected } \\
\text { Count }\end{array}$ & 10.0 & 12.0 & 22.0 \\
\hline & & $\%$ Of Total & $45.5 \%$ & $54.5 \%$ & $100.0 \%$ \\
\hline \multirow[t]{12}{*}{$\begin{array}{l}\text { Health Status } \\
\text { Outcomes }\end{array}$} & Standardized & Count & 7 & 2 & 9 \\
\hline & & $\begin{array}{l}\text { Expected } \\
\text { Count }\end{array}$ & 6.4 & 2.6 & 9.0 \\
\hline & & $\%$ Of Total & $41.2 \%$ & $11.8 \%$ & $52.9 \%$ \\
\hline & Tailored & Count & 3 & 1 & 4 \\
\hline & & $\begin{array}{l}\text { Expected } \\
\text { Count }\end{array}$ & 2.8 & 1.2 & 4.0 \\
\hline & & $\%$ Of Total & $17.6 \%$ & $5.9 \%$ & $23.5 \%$ \\
\hline & Combination & Count & 2 & 2 & 4 \\
\hline & & $\begin{array}{l}\text { Expected } \\
\text { Count }\end{array}$ & 2.8 & 1.2 & 4.0 \\
\hline & & $\%$ Of Total & $11.8 \%$ & $11.8 \%$ & $23.5 \%$ \\
\hline & Total & Count & 12 & 5 & 17 \\
\hline & & $\begin{array}{l}\text { Expected } \\
\text { Count }\end{array}$ & 12.0 & 5.0 & 17.0 \\
\hline & & $\%$ Of Total & $70.6 \%$ & $29.4 \%$ & $100.0 \%$ \\
\hline \multirow[t]{12}{*}{ Self-Rated Outcomes } & Standardized & Count & 4 & 4 & 8 \\
\hline & & $\begin{array}{l}\text { Expected } \\
\text { Count }\end{array}$ & 3.1 & 4.9 & 8.0 \\
\hline & & $\%$ Of Total & $30.8 \%$ & $30.8 \%$ & $61.5 \%$ \\
\hline & Tailored & Count & 0 & 1 & 1 \\
\hline & & $\begin{array}{l}\text { Expected } \\
\text { Count }\end{array}$ & 0.4 & 0.6 & 1.0 \\
\hline & & $\%$ Of Total & $0 \%$ & $7.7 \%$ & $7.7 \%$ \\
\hline & Combination & Count & 1 & 3 & 4 \\
\hline & & $\begin{array}{l}\text { Expected } \\
\text { Count }\end{array}$ & 1.5 & 2.5 & 4.0 \\
\hline & & $\%$ Of Total & $7.7 \%$ & $23.1 \%$ & $30.8 \%$ \\
\hline & Total & Count & 5 & 8 & 13 \\
\hline & & $\begin{array}{l}\text { Expected } \\
\text { Count }\end{array}$ & 5.0 & 8.0 & 13 \\
\hline & & $\%$ Of Total & $38.5 \%$ & $61.5 \%$ & $100.0 \%$ \\
\hline
\end{tabular}


Non-Significant Findings Health Service Utilization: $\left[\chi 2_{(2)}=3.443, p=0.179\right]$.

Non-Significant Findings Health Status Outcomes: $\left[\chi_{(2)}=1.078, p=0.583\right]$.

Non-Significant Findings Self-Rated Outcomes: $\left[\chi_{(2)}=1.381, p=0.501\right]$. 


\section{Appendix R: Analysis of Variance Results}

Health-Service Utilization: The Duration of the Intervention

\begin{tabular}{|c|c|c|c|c|c|c|}
\hline & $\begin{array}{l}\text { Sum of } \\
\text { Squares }\end{array}$ & & $\begin{array}{l}\text { Mean } \\
\text { Square }\end{array}$ & $\mathbf{F}$ & & Sig. \\
\hline Between Group & 0.223 & 2 & 0.112 & & 0.309 & 0.738 \\
\hline Within Groups & 6.868 & 19 & 0.361 & & & \\
\hline Total & 7.091 & 21 & & & & \\
\hline
\end{tabular}

$[F(2,21)=0.309, p=0.738]$.

Health Status Outcomes: The Duration of the Intervention

\begin{tabular}{|lr|rrrr|} 
& Sum of Squares & df & Mean Square & F & Sig. \\
\hline Between Group & 4.960 & 2 & 2.480 & 4.344 & $* 0.029$ \\
\hline Within Groups & 10.278 & 18 & 0.571 & & \\
\hline Total & 15.238 & 20 & & & \\
\hline
\end{tabular}

* Statistical Significance $[\mathrm{F}(2,20)=4.344, p=0.029]$.

Self-Rated Outcomes: The Duration of the Intervention

\begin{tabular}{|lcrrrr|} 
& Sum of Squares & df & Mean Square & F & \multicolumn{1}{c|}{ Sig. } \\
\hline Between Group & 0.202 & 2 & 0.101 & 0.370 & 0.701 \\
\hline Within Groups & 2.464 & 9 & 0.274 & & \\
\hline Total & 2.667 & 11 & & & \\
\hline
\end{tabular}

$[F(2,11)=0.370, p=0.701]$

Health Status Outcomes: The Number of Intervention Components

\begin{tabular}{|lc|rrrr|} 
& Sum of Squares & df & Mean Square & F & Sig. \\
\hline Between Group & 1.198 & 2 & 0.599 & 0.787 & 0.469 \\
\hline Within Groups & 15.236 & 20 & 0.762 & & \\
\hline Total & 16.435 & 22 & & & \\
\hline
\end{tabular}

$[F(2,11)=0.599, p=0.469]$

Self-Rated Outcomes: The Number of Components the Intervention was Comprised of

\begin{tabular}{|c|c|c|c|c|c|}
\hline & $\begin{array}{l}\text { Sum of } \\
\text { Squares }\end{array}$ & df & Mean Square & $\mathbf{F}$ & Sig. \\
\hline Between Group & 0.827 & 2 & 0.413 & 1.838 & 0.209 \\
\hline Within Groups & 2.250 & 10 & 0.225 & & \\
\hline Total & 3.077 & 12 & & & \\
\hline
\end{tabular}

Health-Service Utilization: The Number of Components the Intervention was Comprised of

\begin{tabular}{|c|c|c|c|c|c|}
\hline & $\begin{array}{l}\text { Sum of } \\
\text { Squares }\end{array}$ & df & Mean Square & F & Sig. \\
\hline Between Group & 0.374 & 3 & 0.125 & 0.334 & 0.801 \\
\hline Within Groups & 7.104 & 19 & 0.374 & & \\
\hline Total & 7.478 & 22 & & & \\
\hline
\end{tabular}

$[F(2,11)=0.334, p=0.801]$ 
Self-Rated Outcomes: The Number of Times the Intervention was Delivered/Month (Dose Frequency)

\begin{tabular}{|c|c|c|c|c|c|}
\hline & $\begin{array}{l}\text { Sum of } \\
\text { Squares }\end{array}$ & df & Mean Square & F & Sig. \\
\hline Between Group & 1.077 & 4 & .269 & 1.077 & .428 \\
\hline Within Groups & 2.000 & 8 & .250 & & \\
\hline Total & 3.077 & 12 & & & \\
\hline
\end{tabular}

Health Status Outcomes: The Number of Times the Intervention was Delivered/Month (Dose Frequency)

\begin{tabular}{|c|c|c|c|c|c|}
\hline & $\begin{array}{l}\text { Sum of } \\
\text { Squares }\end{array}$ & df & Mean Square & $\mathbf{F}$ & Sig. \\
\hline Between Group & 5.713 & 6 & .952 & 1.421 & .267 \\
\hline Within Groups & 10.722 & 16 & .670 & & \\
\hline Total & 16.435 & 22 & & & \\
\hline
\end{tabular}

$[F(6,22)=1.421, p=0.267]$,

Health-Service Utilization: The Number of Times the Intervention was Delivered/Month (Dose Frequency)

\begin{tabular}{|c|c|c|c|c|c|}
\hline & $\begin{array}{l}\text { Sum of } \\
\text { Squares }\end{array}$ & df & Mean Square & F & Sig. \\
\hline Between Group & 3.278 & 6 & .546 & 2.081 & .113 \\
\hline Within Groups & 4.200 & 16 & .262 & & \\
\hline Total & 7.478 & 22 & & & \\
\hline
\end{tabular}

$[F(6,22)=2.081, p=0.113]$.

Self-Rated Outcomes: The Length of the Intervention (In Minutes)

\begin{tabular}{|lr|rrrr|}
\multicolumn{1}{c|}{$\begin{array}{l}\text { Sum of } \\
\text { Squares }\end{array}$} & df & & Mean Square & F & Sig. \\
\hline Between Group & 0.031 & 1 & 0.031 & 0.114 & .742 \\
\hline Within Groups & 3.045 & 40 & 0.623 & & \\
\hline Total & 3.077 & 12 & & & \\
\hline
\end{tabular}

$[F(1,12)=0.114, p=0.742]$.

Health Status Outcomes: The Length of the Intervention (In Minutes)

\begin{tabular}{|lr|rrrr|} 
& \multicolumn{1}{l}{$\begin{array}{l}\text { Sum of } \\
\text { Squares }\end{array}$} & \multicolumn{1}{c|}{ df } & Mean Square & F & \multicolumn{1}{c|}{ Sig. } \\
\hline Between Group & 1.535 & 5 & .307 & .350 & .875 \\
\hline Within Groups & 14.900 & 17 & .876 & & \\
\hline Total & 16.435 & 22 & & & \\
\hline
\end{tabular}

$[F(5,22)=0.350, p=0.875]$.

Health-Service Utilization: The Length of the Intervention (In Minutes)

\begin{tabular}{|c|c|c|c|c|c|}
\hline & $\begin{array}{l}\text { Sum of } \\
\text { Squares }\end{array}$ & df & Mean Square & $\mathbf{F}$ & Sig. \\
\hline Between Group & 1.812 & 5 & .362 & 1.087 & .403 \\
\hline Within Groups & 5.667 & 17 & .333 & & \\
\hline Total & 7.478 & 22 & & & \\
\hline
\end{tabular}

$[F(5,22)=1.087, p=0.403]$. 


\section{References}

Aguado, O., MD, Morcillo, Cèsar, MD, PhD, Delàs, Jordi, MD, PhD, Rennie, M., MD, Bechich, S., MD, Schembari, A., PhD, . . Rosell, Franscesc, MD, PhD. (2010). Long-term implications of a single home-based educational intervention in patients with failure. Heart \& Lung: The Journal of Acute and Critical Care, 39(6), S14-S22. doi:10.1016/j.hrtlng.2010.04.010

Allen, J., Hutchinson, A. M., Brown, R., \& Livingston, P. M. (2014). Quality care outcomes following transitional care interventions for older people from hospital to home: A systematic review. BMC Health Services Research, 14(1), 346. doi:10.1186/1472-6963$14-346$

Allen-Duck, A., Robinson, J. C., \& Stewart, M. W. (2017). Healthcare Quality: A Concept Analysis. Nursing Forum, 52(4), 377-386. doi:10.1111/nuf.12207

Altfeld, S. J., Shier, G. E., Rooney, M., Johnson, T. J., Golden, R. L., Karavolos, K., ... Perry, A. J. (2013). Effects of an Enhanced Discharge Planning Intervention for Hospitalized Older Adults: A Randomized Trial. Gerontologist, 53(3), 430-440. https://doiorg.ezproxy.lib.ryerson.ca/geront/gns109

Basger, B. J., Moles, R. J., \& Chen, T. F. (2015). Impact of an enhanced pharmacy discharge service on prescribing appropriateness criteria: A randomised controlled trial. International Journal of Clinical Pharmacy, 37(6), 1194-1205. doi:10.1007/s11096-0150186-0

Beck, A., Andersen, U., Leedo, E., Jensen, L., Martins, K., Quvang, M., . . Rønholt, F. (2015). Does adding a dietician to the liaison team after discharge of geriatric patients improve nutritional outcome: A randomised controlled trial. Clinical Rehabilitation, 29(11), 11171128. doi:10.1177/0269215514564700 
Beck, A. M., Kjær, S., Hansen, B. S., Storm, R. L., Thal-Jantzen, K., \& Bitz, C. (2013). Followup home visits with registered dietitians have a positive effect on the functional and nutritional status of geriatric medical patients after discharge: A randomized controlled trial. Clinical Rehabilitation, 27(6), 483-493. doi:10.1177/0269215512469384

Beelen, J., de Roos, N. M., \& de Groot, Lisette C P G M. (2017). A 12-week intervention with protein-enriched foods and drinks improved protein intake but not physical performance of older patients during the first 6 months after hospital release: A randomised controlled trial. The British Journal of Nutrition, 117(11). doi:10.1017/S0007114517001477

Berglund, H., Hasson, H., Kjellgren, K., \& Wilhelmson, K. (2015). Effects of a continuum of care intervention on frail older persons' life satisfaction: a randomized controlled study. Journal of Clinical Nursing, 24(7/8), 1079-1090. https://doiorg.ezproxy.lib.ryerson.ca/10.1111/jocn.12699

Berglund, H., Wilhelmson, K., Blomberg, S., Dunér, A., Hasson, H., Kjellgren, K. I., .. . Institute of Medicine, Department of Public Health and Community Medicine. (2013). Older people's views of quality of care: A randomised controlled study of continuum of care. Journal of Clinical Nursing, 22(19-20)

Brovold, T., Skelton, D. A., \& Bergland, A. (2013). Older adults recently discharged from the hospital: Effect of aerobic interval exercise on health-related quality of life, physical fitness, and physical activity. Journal of the American Geriatrics Society, 61(9), 15801585. doi:10.1111/jgs.12400

Burke, R. E., Kripalani, S., Vasilevskis, E. E., \& Schnipper, J. L. (2013). Moving beyond readmission penalties: Creating an ideal process to improve transitional care. Journal of Hospital Medicine, 8(2), 102-109. doi:10.1002/jhm.1990 
Burke, R. E., Guo, R., Prochazka, A. V., \& Misky, G. J. (2014). Identifying keys to success in reducing readmissions using the ideal transitions in care framework. BMC Health Services Research, 14(1), 423. doi:10.1186/1472-6963-14-423

Burns N, Grove S, (2005). Understanding Nursing Research, 5th edition. Toronto: Saunders.

Canadian Institute for Health Information (2011). Seniors and the health care system: What is the impact of multiple chronic conditions? Canadian Institute for Health Information, Retrieved from https://secure.cihi.ca/free_products/air-chronic_disease_aib_en.pdf

Canadian Institute for Health Information (2017). Seniors in transition: Exploring pathways across the care continuum. Canadian Institute for Health Information, Retrieved from https://www.cihi.ca/sites/default/files/document/seniors-in-transition-report-2017-en.pdf

Canadian Medical Association. (2013). Health and health care for an aging population. Canadian Medical Association, Retrieved from https://www.cma.ca/Assets/assetslibrary/document/en/advocacy/policyresearch/CMA_Policy_Health_and_Health_Care_fo r_an_Aging-Population_PD14-03-e.pdf

Canadian Medical Association. (2016). The state of seniors' health care in Canada. Canadian Medical Association, Retrieved from https://www.cma.ca/En/Lists/Medias/thestate-of-seniors-health-care-in-canada-september-2016.pdf

Chow, S. K. Y., \& Wong, F. K. Y. (2014). A randomized controlled trial of a nurse-led case management programme for hospital-discharged older adults with co-morbidities. Journal of Advanced Nursing, 70(10), 2257-2271. doi:10.1111/jan.12375

Clemson, L., Lannin, N. A., Wales, K., Salkeld, G., Rubenstein, L., Gitlin, L., ... Cameron, I. D. (2016). Occupational therapy predischarge home visits in acute hospital care: A 
randomized trial. Journal of the American Geriatrics Society, 64(10), 2019-2026. https://doi-org.ezproxy.lib.ryerson.ca/10.1111/jgs.14287

Coleman, E. A., Boult, C., \& American Geriatrics Society Health Care Systems Committee. (2003). Improving the quality of transitional care for persons with complex care needs. Journal of the American Geriatrics Society, 51(4), 556-557. doi:10.1046/j.15325415.2003.51186.x

Courtney, M. D., Edwards, H. E., Chang, A. M., Parker, A. W., Finlayson, K., Bradbury, C., \& Nielsen, Z. (2012). Improved functional ability and independence in activities of daily living for older adults at high risk of hospital readmission: A randomized controlled trial. Journal of Evaluation in Clinical Practice, 18(1), 128.

Courtney, M. D., Edwards, H. E., Chang, A. M., Parker, A. W., Finlayson, K., \& Hamilton, K. (2011). A randomised controlled trial to prevent hospital readmissions and loss of functional ability in high risk older adults: A study protocol. BMC Health Services Research, 11(1), 202-202. doi:10.1186/1472-6963-11-202

Courtney, M., Edwards, H., Chang, A., Parker, A., Finlayson, K., \& Hamilton, K. (2009). Fewer emergency readmissions and better quality of life for older adults at risk of hospital readmission: A randomized controlled trial to determine the effectiveness of a 24 -week exercise and telephone follow-up program. Journal of the American Geriatrics Society, 57(3), 395. doi:10.1111/j.1532-5415.2009.02138.x

Covinsky, K. E., Pierluissi, E., \& Johnston, C. B. (2011). Hospitalization-associated disability: "she was probably able to ambulate, but I'm not sure". Jama, 306(16), 1782. 
DeCoster, V., Ehlman, K., \& Conners, C. (2013). Factors contributing to readmission of seniors into acute care hospitals. Educational Gerontology, 39(12), 878-887. doi:10.1080/03601277.2013.767615

Di Monaco, M., De Toma, E., Gardin, L., Giordano, S., Castiglioni, C., \& Vallero, F. (2015). A single postdischarge telephone call by an occupational therapist does not reduce the risk of falling in women after hip fracture: A randomized controlled trial. European Journal of Physical and Rehabilitation Medicine, 51(1), 15.

Ebrahimi, Z., Eklund, K., Dahlin-Ivanoff, S., Jakobsson, A., \& Wilhelmson, K. (2017). Effects of a continuum of care intervention on frail elders' self-rated health, experiences of security/safety and symptoms: A randomised controlled trial. Nordic Journal of Nursing Research, 37(1), 33-43. doi:10.1177/2057158516668710

Effective Public Health Practice Project. (2010). Quality assessment tool for quantitative studies. Hamilton, ON: Health Practice Project. Retrieved from: https://merst.ca/wpcontent/uploads/2018/02/quality-assessment-tool_2010.pdf

Ekelund, C., \& Eklund, K. (2015). Longitudinal effects on self-determination in the RCT "Continuum of care for frail elderly people." Quality in Ageing \& Older Adults, 16(3), 165-176. doi:10.1108/QAOA-12-2014-0045

Eklund, K., Wilhelmson, K., Gustafsson, H., Landahl, S., Dahlin-Ivanoff, S., Institutionen för hälsovetenskaper, . . . Lunds universitet. (2013). One-year outcome of frailty indicators and activities of daily living following the randomised controlled trial: "continuum of care for frail older people". BMC Geriatrics, 13(76). doi:10.1186/1471-2318-13-76

Finlayson, K., Chang, A. M., Courtney, M. D., Edwards, H. E., Parker, A. W., Hamilton, K., . . . O'Brien, J. (2018). Transitional care interventions reduce unplanned hospital 
readmissions in high-risk older adults. BMC Health Services Research, 18(1), 956. doi:10.1186/s12913-018-3771-9

Fox, M. T., Persaud, M., Maimets, I., Brooks, D., O'Brien, K., \& Tregunno, D. (2013). Effectiveness of early discharge planning in acutely ill or injured hospitalized older adults: A systematic review and meta-analysis. BMC Geriatrics, 13(1), doi:70-70. $10.11 \mathrm{~h} 86 / 1471-2318-13-70$

Fuster, V. (2017). Changing demographics: A new approach to global health care due to the aging population. Journal of the American College of Cardiology, 69(24), doi:3002. 10.1016/j.jacc.2017.05.013

Grant, M. J., \& Booth, A. (2009). A typology of reviews: An analysis of 14 review types and associated methodologies. Health Information \& Libraries Journal, 26(2), 91-108. doi:10.1111/j.1471-1842.2009.00848.x

Gray, J., Grove, S. \& Sutherland, S. (2017). Burns and Groves's the practice of nursing research: Appraisal, synthesis and generation of evidence (8th edition). St. Louis: Elsevier.

Gurwitz, J. H., Field, T. S., Ogarek, J., Tjia, J., Cutrona, S. L., Harrold, L. R., . . Garber, L. (2014). An electronic health record-based intervention to increase follow-up office visits and decrease rehospitalization in older adults. Journal of the American Geriatrics Society, 62(5), 865-871. doi:10.1111/jgs.12798

Haines, T. P., Russell, T., Brauer, S. G., Erwin, S., Lane, P., Urry, S., . . Condie, P. (2009). Effectiveness of a video-based exercise programme to reduce falls and improve healthrelated quality of life among older adults discharged from hospital: A pilot randomized 
controlled trial. Clinical Rehabilitation, 23(11), 973-985.

doi: $10.1177 / 0269215509338998$

Halfon, P., Eggli, Y., Van Melle, G., \& Vagnair, A. (2001). Risk of falls for hospitalized patients: A predictive model based on routinely available data. Journal of Clinical Epidemiology, 54(12), 1258-1266. 10.1016/S0895-4356(01)00406-1

Harden, A., \& Thomas, J. (2005). Methodological issues in combining diverse study types in systematic reviews. International Journal of Social Research Methodology, 8(3), 257271. doi:10.1080/13645570500155078

Hawe, P., Shiell, A., \& Riley, T. (2004). Complex interventions: How "out of control" can a randomised controlled trial be? BMJ: British Medical Journal, 328(7455), 1561-1563. doi:10.1136/bmj.328.7455.1561

Health Quality Ontario. (2018) The Health Quality Ontario Indicator Library. Retreived from http://indicatorlibrary.hqontario.ca/Indicator/Search/EN

Hirshon, J. M., Risko, N., Calvello, E. J. B., Stewart de Ramirez, S., Narayan, M., Theodosis, C., ... Acute Care Research Collaborative at the University of Maryland Global Health Initiative. (2013). Health systems and services: The role of acute care. Bulletin of the World Health Organization, 91(5), 386. doi:10.2471/BLT.12.112664

Holly, C., Salmond, S. W., Saimbert, M., \& Ebooks Corporation. (2012). Comprehensive systematic review for advanced nursing practice: Springer Pub.

Hsiao-Mei, C., Yi-Hsuan, T., \& Ching-Min, C. (2017). Effect of Continuity of Care on Quality of Life in Older Adults With Chronic Diseases: A Meta-Analysis. Clinical Nursing Research, 26(3), 266-284. doi:10.1177/1054773815625467 
Hyde, C. J., Robert, I. E., \& Sinclair, A. J. (2000). The effects of supporting discharge from hospital to home in older people. Age and Ageing, 29(3), 271.

Kansagara, D., Chiovaro, J. C., Kagen, D., Jencks, S., Rhyne, K., O'Neil, M., . . Englander, H. (2016). So many options, where do we start? an overview of the care transitions literature. Journal of Hospital Medicine, 11(3), 221-230. 10.1002/jhm.2502

Kim, H., \& Thyer, B. A. (2015). Does transitional care prevent older adults from rehospitalization? A review. Journal of Evidence-Informed Social Work, 12(3), 261-271. doi:10.1080/15433714.2013.827140

Kirk, C. (2014). Telephone follow-up of older people after hospital admissions. Age and Ageing, 43(suppl 2), doi:10.1093/ageing/afu124.28

Koehler, B. E., Richter, K. M., Youngblood, L., Cohen, B. A., Prengler, I. D., Cheng, D., \& Masica, A. L. (2009). Reduction of 30-day postdischarge hospital readmission or emergency department (ED) visit rates in high-risk elderly medical patients through delivery of a targeted care bundle. Journal of Hospital Medicine, 4(4), 211-218. doi:10.1002/jhm.427

Kwok, T., Lee, J., Woo, J., Lee, D. T., \& Griffith, S. (2008). A randomized controlled trial of a community nurse-supported hospital discharge programme in older patients with chronic heart failure. Journal of Clinical Nursing, 17(1), 109-117. doi:10.1111/j.13652702.2007.01978.x

Le Berre, M., Maimon, G., Sourial, N., Guériton, M., \& Vedel, I. (2017). Impact of Transitional Care Services for Chronically Ill Older Patients: A Systematic Evidence Review. Journal of The American Geriatrics Society, 65(7), 1597-1608. doi:10.1111/jgs.14828 
Lindegaard Pedersen, J., Pedersen, P., \& Damsgaard, E. (2017). Nutritional follow-up after discharge prevents readmission to hospital - A randomized clinical trial. Journal of Nutrition, Health \& Aging, 21(1), 75-82. doi:10.1007/s12603-016-0745-7

Li Xueyu, Yu Hao, Xu Shunlin, Li Rongbin, \& Gao Yuan. (2017). Effects of Low-Intensity Exercise in Older Adults With Chronic Heart Failure During the Transitional Period From Hospital to Home in China: A Randomized Controlled Trial. Research in Gerontological Nursing, 10(3), 121-128. doi:10.3928/19404921-20170411-02

Lowthian, J. (2017). How do we optimise care transition of frail older people?. Age \& Ageing, 46(1), 2-4. doi:10.1093/ageing/afw171

Moher D, Liberati A, Tetzlaff J, Altman DG, The PRISMA Group (2009). Preferred reporting ttems for systematic reviews and meta-analyses: The PRISMA Statement. PLoS Med 6(7): e1000097. doi:10.1371/journal.pmed1000097

Morrow, E. M., \& Nicholson, C. (2016). Carer engagement in the hospital care of older people: An integrative literature review. International Journal of Older People Nursing, 11(4), 298-314. doi:10.1111/opn.12117

Mora, K., Dorrejo, X. M., Carreon, K. M., \& Butt, S. (2017). Nurse practitioner-led transitional care interventions: An integrative review. Journal of The American Association Of Nurse Practitioners, 29(12), 773-790. doi:10.1002/2327-6924.12509

Murray, G. R., Cameron, I. D., \& Cumming, R. G. (2007). The consequences of falls in acute and subacute hospitals in Australia that cause proximal femoral fractures: Consequences of hospital-acquired pff. Journal of the American Geriatrics Society, 55(4), 577-582. doi:10.1111/j.1532-5415.2007.01102.x 
Naylor, M. D. (2012). Advancing high value transitional care: The central role of nursing and its leadership. Nursing Administration Quarterly, 36(2), 115.

Naylor, M. D., Bowles, K. H., \& Brooten, D. (2000). Patient problems and advanced practice nurse interventions during transitional care. Public Health Nursing, 17(2), 94-102. doi:10.1046/j.1525-1446.2000.00094.x

Naylor, M. D., Bowles, K. H., McCauley, K. M., Maccoy, M. C., Maislin, G., Pauly, M. V., \& Krakauer, R. (2011). High-value transitional care: Translation of research into practice: High-value transitional care. Journal of Evaluation in Clinical Practice, doi:10.1111/j.1365-2753.2011.01659.x

Naylor, M. D., Feldman, P. H., Keating, S., Koren, M. J., Kurtzman, E. T., Maccoy, M. C., \& Krakauer, R. (2009). Translating research into practice: Transitional care for older adults. Journal of Evaluation in Clinical Practice, 15(6), 1164-1170. doi:10.1111/j.13652753.2009.01308.x

Naylor, M. D., Kurtzman, E. T., \& Pauly, M. V. (2009). Transitions of elders between long-term care and hospitals. Policy, Politics, \& Nursing Practice, 10(3), 187-194. doi:10.1177/1527154409355710

Ontario Association of Non-Profit Homes and Services for Seniors. (2016). Improving seniors services in Ontario. OANHSS, Retrieved from https://www.pshsa.ca/wpcontent/uploads/2016/06/OANHSS-Capacity-Planning.pdf

Parry, C., Coleman, E. A., Smith, J. D., Frank, J., \& Kramer, A. M. (2003). The care transitions intervention: A patient-centered approach to ensuring effective transfers between sites of geriatric care. Home Health Care Services Quarterly, 22(3), 1-17. doi:10.1300/J027v22n03_01 
Parry C, Min S, Chugh A, Chalmers S, \& Coleman EA. (2009). Further application of the care transitions intervention: results of a randomized controlled trial conducted in a fee-forservice setting. Home Health Care Services Quarterly, 28(2/3), 84-99. https://doiorg.ezproxy.lib.ryerson.ca/10.1080/0162142090315592

Pedersen, J. L., Pedersen, P. U., \& Damsgaard, E. M. (2016). Early nutritional follow-up after discharge prevents deterioration of ADL functions in malnourished, independent, geriatric patients who live alone-A randomized clinical trial. The Journal of Nutrition, Health \& Aging, 20(8), 845-853. doi:10.1007/s12603-015-0629-2

Pincus, K. (2013). Transitional care management services: Optimizing medication reconciliation to improve the care of older adults. Journal of Gerontological Nursing, 39(10), 10-15. doi:10.3928/00989134-20130827-03

Rasmussen, R. S., Østergaard, A., Kjær, P., Skerris, A., Skou, C., Christoffersen, J., ... Overgaard, K. (2016). Stroke rehabilitation at home before and after discharge reduced disability and improved quality of life: A randomised controlled trial. Clinical Rehabilitation, 30(3), 225-236. doi:10.1177/0269215515575165

Rockers, P. C., Røttingen, J., Shemilt, I., Tugwell, P., \& Bärnighausen, T. (2015). Inclusion of quasi-experimental studies in systematic reviews of health systems research. Health Policy, 119(4), 511-521. doi:10.1016/j.healthpol.2014.10.006

Rockwood, K., Song, X., MacKnight, C., Bergman, H., Hogan, D. B., McDowell, I., \& Mitnitski, A. (2005). A global clinical measure of fitness and frailty in elderly people. CMAJ: Canadian Medical Association Journal, 173(5), doi:489495.10.1503/cmaj.050051 
Rytter, L., Jakobsen, H. N., Rønholt, F., Hammer, A. V., Andreasen, A. H., Nissen, A., \& Kjellberg, J. (2010). Comprehensive discharge follow-up in patients' homes by GPs and district nurses of elderly patients. A randomized controlled trial. Scandinavian Journal of Primary Health Care, 28(3), 146-153. doi:10.3109/02813431003764466

Saleh, S. S., Freire, C., Morris-Dickinson, G., \& Shannon, T. (2012). An effectiveness and costbenefit analysis of a hospital-based discharge transition program for elderly medicare recipients. Journal of the American Geriatrics Society, 60(6), 1051-1056. doi:10.1111/j.1532-5415.2012.03992.x

Shepperd, S., Lannin, N. A., Clemson, L. M., McCluskey, A., Cameron, I. D., \& Barras, S. L. (2013). Discharge planning from hospital to home. The Cochrane Database of Systematic Reviews, (1), CD000313.

Sidani, S. (2015). Health intervention research: Understanding research design \& methods. SAGE Publications Ltd.

Sidani, S., \& Braden, C.J. (1998). Evaluating nursing interventions. A theory driven approach. Thousand Oaks, CA: Sage

Sidani, S., \& Braden, C. J. (2011). Design, evaluation, and translation of nursing interventions. Wiley-Blackwell.

Siddiqi, N., House, A. O., \& Holmes, J. D. (2006). Occurrence and outcome of delirium in medical in-patients: A systematic literature review. Age and Ageing, 35(4), doi:350-364. 10.1093/ageing/afl005

Stevens-Lapsley, J. E., Loyd, B. J., Falvey, J. R., Figiel, G. J., Kittelson, A. J., Cumbler, E. U., \& Mangione, K. K. (2016). Progressive multi-component home-based physical therapy for 
deconditioned older adults following acute hospitalization: A pilot randomized controlled trial. Clinical Rehabilitation, 30(8), 776-785. doi:10.1177/0269215515603219

Thygesen, L. C., Fokdal, S., Gjørup, T., Taylor, R. S., Zwisler, A., Prevention of Early Readmission Research Group, \& on behalf of the Prevention of Early Readmission Research Group. (2015). Can municipality-based post-discharge follow-up visits including a general practitioner reduce early readmission among the fragile elderly (65+ years old)? A randomized controlled trial. Scandinavian Journal of Primary Health Care, 33(2), 65-73. doi:10.3109/02813432.2015.1041831

Toye, C., Parsons, R., Slatyer, S., Aoun, S. M., Moorin, R., Osseiran-Moisson, R., \& Hill, K. D. (2016). Outcomes for family carers of a nurse-delivered hospital discharge intervention for older people (the further enabling care at home program): Single blind randomised controlled trial. International Journal of Nursing Studies, 64(32-41). doi:10.1016/j.jinurstu.2016.09.012

Ueda, T., Higuchi, Y., Imaoka, M., Todo, E., Kitagawa, T., \& Ando, S. (2017). Tailored education program using home floor plans for falls prevention in discharged older patients: A pilot randomized controlled trial. Archives of Gerontology and Geriatrics, 71 (9-13). doi:10.1016/j.archger.2017.02.010

Vogler, Constance M., MBBS, FRACP, PhD, Menant, J. C., PhD, Sherrington, C., PhD, Ogle, Susan J., MBBS, FRACP, \& Lord, Stephen R., PhD, DSc. (2012). Evidence of detraining after 12-week home-based exercise programs designed to reduce fall-risk factors in older people recently discharged from hospital. Archives of Physical Medicine and Rehabilitation, 93(10), 1685-1691. doi:10.1016/j.apmr.2012.03.033 
Voils, C. I., Chang, Y., Crandell, J., Leeman, J., Sandelowski, M., \& Maciejewski, M. L. (2012). Informing the dose of interventions in randomized trials. Contemporary Clinical Trials, 33(6), 1225-1230. doi:10.1016/j.cct.2012.07.011

Wong, F. K. Y., Chow, S. K. Y., Chan, T. M. F., \& Tam, S. K. F. (2014). Comparison of effects between home visits with telephone calls and telephone calls only for transitional discharge support: A randomised controlled trial. Age and Ageing, 43(1), 91-97. doi:10.1093/ageing/aft123

Wong, F. K., Ho, M. M., Yeung, S., Tam, S. K., \& Chow, S. K. (2011). Effects of a health-social partnership transitional program on hospital readmission: A randomized controlled trial. Social Science \& Medicine, 73(7), 960-969. doi:10.1016/j.socscimed.2011.06.036

Wong, K. S., Ryan, D. P., \& Liu, B. A. (2014). A System-Wide analysis using a Senior-Friendly hospital framework identifies current practices and opportunities for improvement in the care of hospitalized older adults. Journal of the American Geriatrics Society, 62(11), doi:2163-2170. 10.1111/jgs.13097

World Health Organization, (2018). Proposed working definition of an older person in Africa for the MDS Project. World Health Organization, Retrieved from http://www.who.int /healthinfo/survey/ageingdefnolder/en/

Xueyu Li, Shunlin Xu, Lijuan Zhou, Rongbin Li, \& Jianrong Wang. (2015). Home-based exercise in older adults recently discharged from the hospital for cardiovascular disease in China. Nursing Research, 64(4), 246-255. doi:10.1097/NNR.0000000000000102

Yu, D. S. F., Lee, D. T. F., Stewart, S., Thompson, D. R., Choi, K., \& Yu, C. (2015). Effect of nurse-implemented transitional care for Chinese individuals with chronic heart failure in 
Hong Kong: A randomized controlled trial. Journal of the American Geriatrics Society,

63(8), 1583-1593. doi:10.1111/jgs.13533 